University of Rhode Island

DigitalCommons@URI

Open Access Dissertations

2011

\title{
ACOUSTIC FLUCTUATIONS IN SHALLOW WATER DUE TO NONLINEAR INTERNAL WAVES
}

Georges Albert Dossot

University of Rhode Island, georges.dossot@gmail.com

Follow this and additional works at: https://digitalcommons.uri.edu/oa_diss

\section{Recommended Citation}

Dossot, Georges Albert, "ACOUSTIC FLUCTUATIONS IN SHALLOW WATER DUE TO NONLINEAR INTERNAL WAVES" (2011). Open Access Dissertations. Paper 116.

https://digitalcommons.uri.edu/oa_diss/116

This Dissertation is brought to you for free and open access by DigitalCommons@URI. It has been accepted for inclusion in Open Access Dissertations by an authorized administrator of DigitalCommons@URI. For more information, please contact digitalcommons-group@uri.edu. 
ACOUSTIC FLUCTUATIONS IN SHALLOW WATER DUE TO NONLINEAR INTERNAL WAVES

BY

GEORGES ALBERT DOSSOT

A DISSERTATION SUBMITTED IN PARTIAL FULFILLMENT OF THE

REQUIREMENTS FOR THE DEGREE OF

DOCTOR OF PHILOSOPHY

IN

OCEAN ENGINEERING

UNIVERSITY OF RHODE ISLAND

2011 


\section{DOCTOR OF PHILOSOPHY IN OCEAN ENGINEERING DISSERTATION \\ OF}

\section{GEORGES ALBERT DOSSOT}

\section{APPROVED:}

Dissertation Committee:

Major Professor JAMES H. MILLER

MOHSEN BADIEY

JAMES F. LYNCH

GOPU R. POTY

H. THOMAS ROSSBY

KEVIN B. SMITH

NASSER H. ZAWIA

DEAN OF THE GRADUATE SCHOOL

UNIVERSITY OF RHODE ISLAND

2011 


\begin{abstract}
This study examines underwater acoustic propagation in a shallow water environment, concentrating upon the impact of nonlinear internal waves. During internal wave activity, acoustic signals can fluctuate significantly due to complex three-dimensional multi-mode and multipath interference effects. Experimental measurements from the Shallow Water '06 experiment provide oceanographic and acoustic data during instances where the acoustic track is nearly parallel to an approaching internal wave train. Distinct events show internal waves modulate the acoustic field substantially. Propagation predictions using the Monterey-Miami Parabolic Equation model simulate an internal wave train moving in a straight-line fashion. Horizontal refraction dominates in the nearly parallel configuration, and three specific scenarios are exemplified in both measured and modeled data: refraction (prior to the internal wave's arrival), defocusing (as a soliton spreads acoustic energy), and focusing (as two solitons create a horizontal sound channel). Normal mode decomposition and statistical analysis provide insight into the temporal and spatial acoustic fluctuations. In the exactly parallel scenario, refraction prior to the wave's arrival is important, but is overshadowed by focusing effects during the wave's passage. At very small angles off-parallel $\left(\sim 1^{\circ}-5^{\circ}\right)$ acoustic modulations are less severe because focusing effects divert energy from the receiver; refraction prior to the wave's arrival becomes a more important factor in this configuration.
\end{abstract}




\section{ACKNOWLEDGMENTS}

Throughout this text I often use the word we. Perhaps this is simply my writing style. I believe many traditional academics would argue it a poor choice, favoring other pronouns such as "one." Yet, the work summarized in the follow pages, while my own, describes a team effort. Reflecting on my graduate studies as a whole, there have been many fine friends, family, colleagues, and mentors that have made tremendously positive impacts on me. They have stood by, even during the arduous challenges that accompany the doctoral process. They have offered me their knowledge, one of the greatest gifts a person can bestow. They have extended kinship and cooked up fond memories, which I will cherish above all.

I am indebted (the good kind!) to the National Defense Science and Engineering Graduate Fellowship, the Office of Naval Research, and the DOSITS program, for financial opportunities that made returning to school realizable, and not a maybe one day idea. Particularly to CDR Ben Reeder, who took a personal interest in my research and ensured I had the resources to complete this work.

Thank you professors and advisors for your mentorship and direction. Jim Lynch, Ying-Tsong Lin, Art Newhall, and the entire WHOI gang, for helping me vector my efforts and giving me the tools to build with. Mohsen Badiey, for an adventure at sea, a bounty of SW06 data, and continued interest in my research. Kevin Smith, for extraordinary coaching and unfettered access to a world-class acoustic model. Gopu Potty and Jim Miller for shouldering the burden of training, teaching, and advising. Gopu for our daily conversations in my progress, for listening to my bouncing ideas, and for all that you have taught me. Jim, for being the most enjoyable advisor a student could wish for, for the fine people you have connected me with, and for your continued guidance...we'll always have Paris!

Somewhere in this process I recruited a bull-pen of friends and colleagues that I could call upon at a moment's notice. At times, it was for a well-needed break, and sometimes it was for something a bit more. To all my graduate student friends, especially those within Ocean Engineering and G.S.O. thanks for the fun times had. A special shout-out 
to Steven Crocker and Bob Barton, while my fellow grad-students, were also great mentors. To my roommates at the Sass, including my "common-law" Leslie, it's been a great place to call home. To Kiersten, meeting my fellow partner in crime has led to the most exciting and fortuitous adventure someone could ever dream of.

I am also afflicted with a large, loving, and overbearing family. I would not change them for the world. Aunts, uncles, cousins, and those family-friends whom I also consider aunts, uncles, and cousins. They are always there - steady, reliable, anxious to spend time with me, and always supportive. To Mom, Dad, and Brother - love and thanks for everything you guys have done. And even though she is no longer with us, I've dedicated this to my grandmother. After all, she taught me my ABCs and 123s, so I guess she's partly to blame in all this.

Thanks to all! It's been a great ride. Although, this is just a quick pit-stop along the way - a scenic vista, and a breath of air. Hop back in! We're in this together - and I think there are still good times ahead and interesting things to be found.

-- G.A.D 
For Grandma Lou! 


\section{PREFACE}

The following dissertation is intended in part for the fulfillment of the requirements set forth by the University of Rhode Island Graduate School and the Department of Ocean Engineering for the degree of Doctorate of Philosophy in Ocean Engineering. The purpose of this work is to better understand acoustic fluctuations due to the presence of nonlinear internal waves (NIWs) in shallow water. Specifically, the case when the NIW front is nearly parallel to the source-receiver path is considered.

This dissertation is presented in manuscript format. Section headings, references, figures, tables, and all other formatting choices follow the American Institute of Physics Style Manual.

Manuscript I is written in a format specific to the Journal of the Acoustical Society of America Electronic Letter (JASA-EL). This format is mandated to be brief, and limits the author to a figure and page count. The purpose of the Electronic Letter is to quickly alert the acoustics community of recent findings, and this letter represents our first results for both measured and modeled data. In the context of this dissertation, it also serves as an introductory text for the following chapters (Manuscripts II and III).

Manuscript II is also written for the Journal of the Acoustical Society of America. It focuses upon measured data taken from the Shallow Water '06 (SW06) experiment. A brief background of the SW06 experiment pertinent to these data is provided. The background includes important oceanographic factors that influenced the acoustic data, focusing upon the presence of NIWs. A more thorough analysis of the experimental results is presented, including a suggested interpretation of the observational data. 
Manuscript III focuses upon acoustic modeling efforts and expands the initial findings shown in Manuscript I. The oceanographic and acoustic data described in Manuscripts I and II lay the foundation for this chapter, serving as the impetus to carry out this portion of the research. Although directly following from the previous chapters, Manuscript III is written as a stand-alone text, intended for the Journal of the Acoustical Society of America. A background of parabolic equation (PE) modeling, and important parameter considerations pertinent to this study, is offered. Repeated model realizations simulate a NIW crossing the sound field, and the synthetic acoustic data is presented. The analysis is expanded to offer general insight into the impact of NIWs upon the sound field.

Three appendices are included to augment the manuscripts. Appendix A provides additional measured data from the SW06 experiment. It also includes further analysis of one specific NIW event, including the effect of noise. Appendix B provides additional considerations to the PE modeling. Appendix C further details the broadband mode filtering approach used to treat the measured acoustic data. 


\section{TABLE OF CONTENTS}

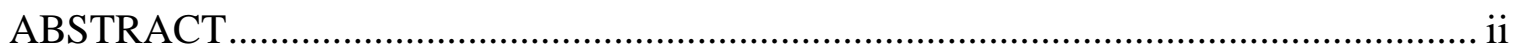

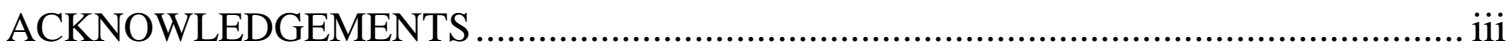

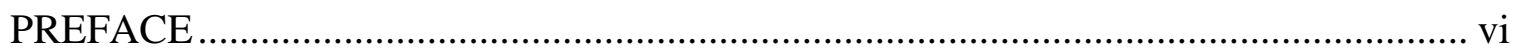

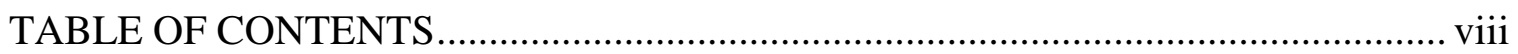

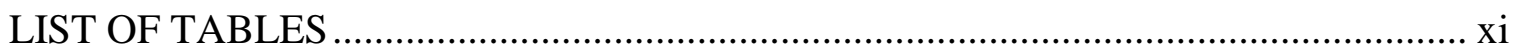

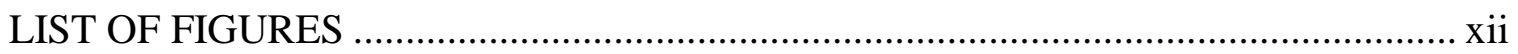

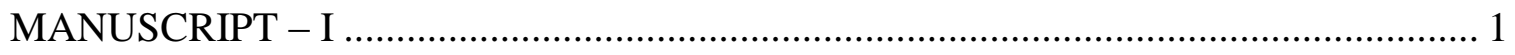

EVIDENCE OF ESCALATING ACOUSTIC INTENSITY PRECEDING A STRONG INTERNAL WAVE EVENT DURING THE SHALLOW WATER '06

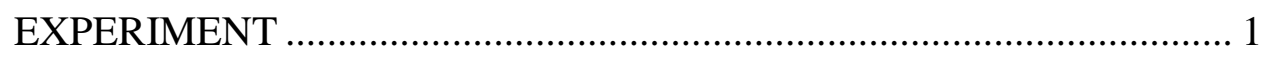

$1.1 \quad$ INTRODUCTION AND BACKGROUND .................................... 3

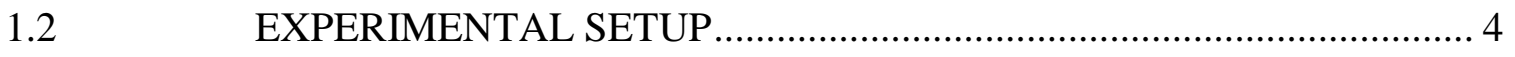

1.3 OCEANOGRAPHIC MEASUREMENTS AND INFERENCES ............... 5

1.4 ACOUSTIC MEASUREMENTS AND EVIDENCE OF HORIZONTAL

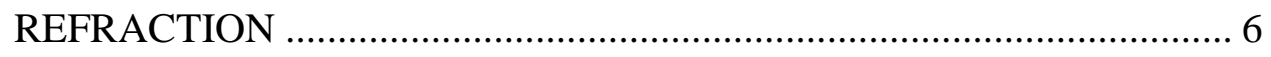

1.5 THREE-DIMENSIONAL PARABOLIC EQUATION MODELING ....... 7

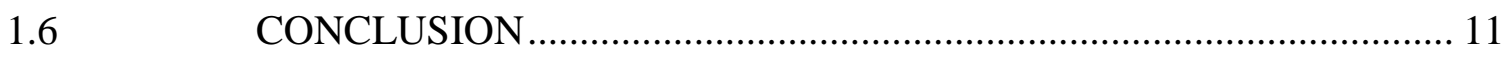

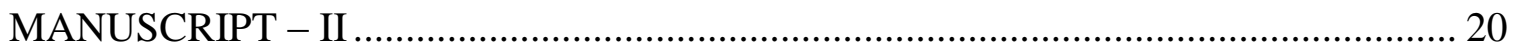

MEASURED INTENSITY FLUCTUATIONS DUE TO HORIZONTAL REFRACTION DURING INTERNAL WAVE ACTIVITY IN THE SHALLOW WATER

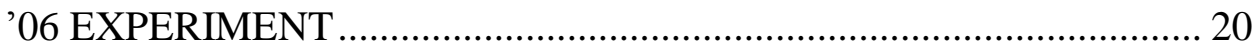


A. Broadband energy metrics .

B. Modal decomposition.

C. Qualitative modeling...

A. Comments upon Event 44 ................................................................ 47

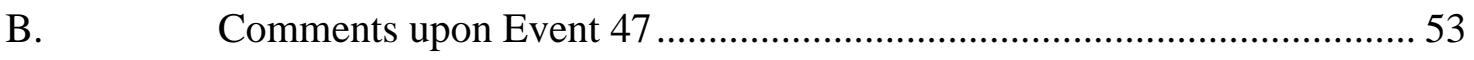

$2.7 \quad$ SUMMARY AND CONCLUSIONS ………........................................ 53

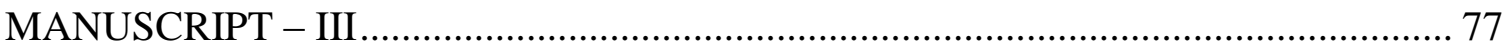

EXAMINING ENERGY FLUCTUATIONS DURING STRONG INTERNAL WAVE ACTIVITY USING A THREE-DIMENSIONAL PARABOLIC

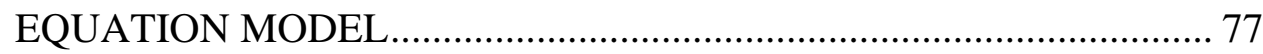

3.1 INTRODUCTION AND BACKGROUND ……………………….... 79

3.2 MMPE THREE-DIMENSIONAL PARABOLIC EQUATION MODEL 81

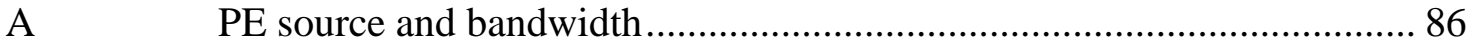

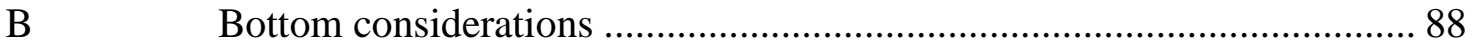

C Modal analysis and reference sound speed sensitivity ……………........ 92 
D "Event 44" internal wave sound speed profile. 95

IMPACTS OF NIW ACTIVITY UPON THE SOUND FIELD ............. 98

A Discrete horizontal refraction scenarios.......................................... 99

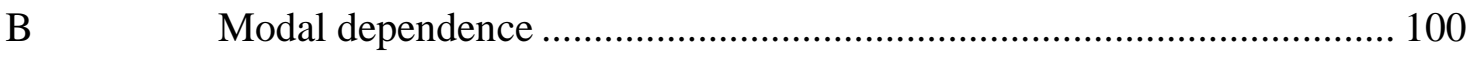

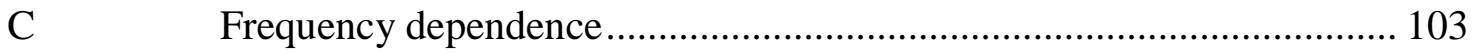

D Simulated time-dependent NIW propagation .................................. 106

E Effects upon small angle variations ......................................... 107

F Time-varying fluctuation regimes............................................. 112

SUMMARY AND CONCLUSIONS ........................................... 114

APPENDIX A. Measured data considerations \& additional NIW events................ 151

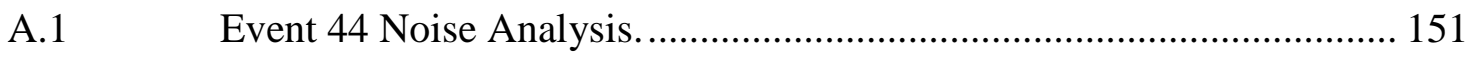

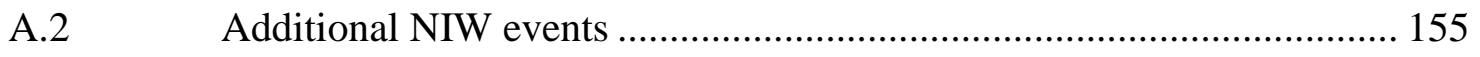

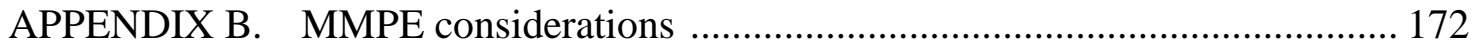

B.1 Frequency sampling considerations …....................................... 172

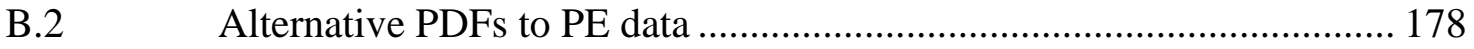

B.3 Modal excitation .................................................................. 178

APPENDIX C. Broadband modal decomposition ........................................... 187

C.1 Problem, assumptions, and theory …......................................... 187

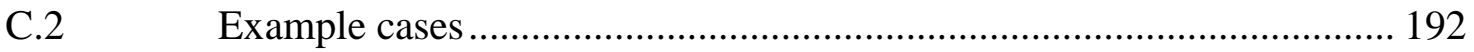




\section{LIST OF TABLES}

\section{Manuscript II}

Table 2.1 Event 44 broadband modal decomposition $(70-325 \mathrm{~Hz})$........................ 52

Table 2.2 Event 44 broadband modal decomposition - lower band (70-200 Hz) .... 52

Table 2.3 Event 44 broadband modal decomposition - upper band (200-325 Hz) .. 52

\section{Manuscript III}

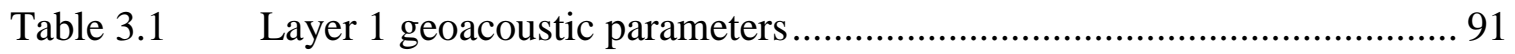

Table $3.2 \quad$ Layer 2 geoacoustic parameters......................................................... 91 


\section{LIST OF FIGURES}

\section{Manuscript I}

Figure 1.1 Close-up of SW06 experimental area and overlaid ship RADAR image. A strong internal wave event propagated nearly parallel to the source (R/V Sharp) and receiver (WHOI VLA) acoustic track. (color online)

Figure 1.2 Acoustic data compared to environmental data. (Top) Measured normalized energy levels received upon each hydrophone of the WHOI VLA versus time. Refraction occurs prior to the NIW's arrival, with possible focusing as the NIW arrives at the acoustic source. A complicated NIW interference regime follows; (Bottom 3 plots) Environmental sensors including R/V Sharp ADCP (at the acoustic source) showing NIW pycnocline, mooring SW32 (midway between source-receiver), and interpolated sound speed at the WHOI receiving VLA, all exhibiting strong internal wave activity. (color online) 18

Figure 1.3 Measured sound speed profile versus depth used for PE simulation (Top left quadrant). Sound speed varies with depth and cross-range, but assumed valid versus range in a "straight-line" approximation. Depth-integrated intensity is shown to the right of the sound speed profile. In this instance, the NIW has not arrived at the source and refraction dominates; (Top right quadrant) NIW has arrived at the source and defocusing dominates; (Bottom left quadrant) NIW traps the source between two solitons and focusing dominates; (Bottom right quadrant) The NIW sound speed profile advances over 100 MMPE simulations to simulate the actual data. Normalized received intensity between 4.5 to 5.5 degrees from the propagating NIW and source-receiver path show escalating intensity prior to the NIW's arrival in the "Refraction" regime. (color online) .19 


\section{Manuscript II}

Figure 2.1 The NIW train can be approximated as series of "sudden" perturbations in the water column, where each soliton alters the modal eigenvalues. Depending on the source-receiver position, the NIW can heavily influence where acoustic energy is directed. The top panel shows a refraction scenario - prior to the NIW reaching the acoustic source. The center panel shows a defocusing scenario -

just as the first soliton reaches the source. The bottom panel shows a focusing scenario - as the source is trapped between two solitons. Soliton perturbations are represented as gray bars for visual interpretation.

Figure 2.2 Time varying intensity fluctuation regimes for a propagating NIW field passing an acoustic source. An acoustic source emits horizontally refracted modal rays at frequency $f$ and mode number $M$. Note: Rays are not depth-dependent, and are plotted at different levels to clarify different regimes. A quiescent regime is followed by a refraction regime prior to the NIW's arrival. As the NIW passes over an acoustic source both focusing and defocusing cause a complicated interference regime.

Figure 2.3 Map of the SW06 experiment, and locations of the R/V Sharp during the two specific NIW Events 44 and 47 (C1B and C1D respectively).

Figure 2.4 A strong internal wave event propagated nearly parallel to the source-receiver acoustic track (R/V Sharp J-15 and WHOI VLA respectively). Ship radar detected the surface expression of the NIW as it approached, passed beneath, and left the R/V Sharp.

Figure 2.5 ADCP plot of Event 44. A strong NIW passed below the R/V Sharp on Aug 14 2006. Top panel shows normalized echo backscatter, showing strong pycnocline depressions occurring between 05:30 and 07:30 GMT. Center panel shows the 
magnitude of vertical particle velocity. Bottom panel shows vertical velocity. (color online).

Figure 2.6 ADCP plot of Event 47. A strong NIW passed below the R/V Sharp on Aug 16 2006. Top panel shows normalized echo backscatter, showing three strong pycnocline depressions. Center panel shows the magnitude of vertical particle velocity. Bottom panel shows vertical velocity. (color online) .66

Figure 2.7 Computed sound speed versus time during CTD "yo-yo." Event 44 arrives at the ship and acoustic source at roughly 05:40 GMT and is marked by a depressed region of faster sound speed, followed by fluctuating sound speed profiles versus time and depth. (color online).

Figure 2.8 CTD measurements of temperature (left), salinity (center), and computed sound speed (right) during event 47 .

Figure 2.9 (Top) Measured normalized energy levels received upon each hydrophone of the WHOI VLA versus time. Refraction occurs prior to the NIW's arrival, with possible focusing as the NIW arrives at the acoustic source. A complicated NIW interference regime follows; (Bottom 3 plots) Environmental sensors including R/V Sharp ADCP (at the acoustic source) showing NIW pycnocline, mooring SW32 (midway between source-receiver), and interpolated sound speed at the WHOI receiving VLA, all exhibiting strong internal wave activity. (color online)

Figure 2.10 Measured normalized energy levels received upon each hydrophone of the WHOI VLA versus time during Event 47. Possible refraction occurs prior to the NIW's arrival, with defocusing and focusing effects as the soliton reaches the acoustic track, and a possible shadow zone following (top). Environmental mooring SW32 was situated midway between the source and receiver and shows evidence of strong soliton activity (bottom). (color online) .70 
Figure 2.11 Point observations are plotted versus transmission number (top left), and the data fits well to a lognormal distribution (top right). Depth dependent variability is plotted using a normalized color scalogram versus transmission number (bottom left), or alternatively shown through a statistical boxplot of received energy upon each hydrophone (bottom right). (color online).............................................. 71

Figure 2.12 Spectrogram showing a received chirp signal upon the eighth hydrophone (at 39 meters deep) of the WHOI VLA (left). This signal is broken down into broadband modal components for modes 1-5 (right panels). Note the "splitting" of the signal can be explained by separate modal arrivals. Color scales across plots are identical. (color online) …........................................................... 72

Figure 2.13 Normalized modal arrivals for the chirp signal shown in Figure 2.12 ............. 73

Figure 2.14 Modal components of intensity point observations for 70-325 Hz (left); the lower frequency spectrum, 70-200 Hz (center); and the upper frequency spectrum, 200$325 \mathrm{~Hz}$ (right). Modes 1-3 dominate energy in the lower frequency band, while modes 3-5 dominate energy in the upper frequency spectrum. (color online)....74

Figure 2.15 Event 44 sound speed profile (left) used as the primary environmental variable in the 3D KRAKEN normal mode code, and is assumed to remain valid across range. The acoustic source is located at the star, simulating a passing NIW. Modal rays are horizontally refracted by the NIW in a refraction, defocus, and focus condition based on the position of the source. (color online).

Figure 2.16 Event 44 is separated into the proposed refraction, focusing, and NIW interference regimes. Refraction effects prior to the NIW's arrival cause similar variability as NIW interference effects. Focusing effects dominate both intensification and signal variability. (color online) .76 


\section{Manuscript III}

Figure 3.1 Over 1,000 two-dimensional PE simulations varied several bottom parameters based upon estimates from previously published inversion values. Depthintegrated intensity versus range shows the influence of these parameters upon bottom attenuation. Four distinct bands became apparent - indicating layer one compressional attenuation, compressional speed, shear speed, and density are the most influential factors. 122

Figure 3.2 The choice of reference sound speed $\left(c_{0}\right)$ can impact the performance of the PE model. The top panel shows a modeled area where roughly half of the input profile contains a NIW, and where refraction effects will dominate. Below is the reference sound speed that would be associated with the $c_{0}$-independent $\left(c_{0 I P E}\right)$ calculation. 123

Figure 3.3 A sensitivity analysis compared the impact of varying $c_{0}$ upon modal decomposition. The choice of $c_{0}$ can more closely align eigenvalues of the Helmholtz equation with those of the wide angle source PE field. Modal decomposition of the PE field is shown in blue, with predicted in red (top). The residuals were used as a measure for goodness of fit. 124

Figure 3.4 A general layout for the acoustic problem of interest. A progressing NIW approaches and crosses the acoustic track such that the soliton wave-fronts are nearly parallel. Horizontal refraction plays a dominant role in this scenario. .. 125

Figure 3.5 Three scenarios showing refraction (left), defocusing (center), and focusing (right). Top panels show the sound speed profile relative to the $100 \mathrm{~Hz}$ acoustic source (marked by a star) used in the PE model; the profile is valid across range. The center panels show depth integrated intensity throughout the three- 
dimensional water column. The bottom panels show transmission loss versus depth $15 \mathrm{~km}$ away from the acoustic source. (color online) 126

Figure 3.6 Modeshapes (for modes 1-5) at each cross-range point in the sound speed profile. The modeshape maxima and minima are pushed downward in the water column at soliton locations. (color online)

Figure 3.7 Depth integrated intensity and modal decomposition of the PE field for modes 15 for a $300 \mathrm{~Hz}$ source. Sound speed input creates a refraction scenario. (color online) 128

Figure 3.8 Depth integrated intensity and modal decomposition of the PE field for modes 610 for a $300 \mathrm{~Hz}$ source. Sound speed input creates a refraction scenario. (color online) 129

Figure 3.9 Depth integrated intensity and modal decomposition of the PE field for modes 15 for a $300 \mathrm{~Hz}$ source. Sound speed input creates defocus scenario. (color online)

Figure 3.10 Depth integrated intensity and modal decomposition of the PE field for modes 610 for a $300 \mathrm{~Hz}$ source. Sound speed input creates defocus scenario. (color online) 131

Figure 3.11 Depth integrated intensity and modal decomposition of the PE field for modes 15 for a $300 \mathrm{~Hz}$ source. Sound speed input creates a focus scenario. (color online)

Figure 3.12 Depth integrated intensity and modal decomposition of the PE field for modes 610 for a $300 \mathrm{~Hz}$ source. Sound speed input creates a focus scenario. (color online) 133

Figure 3.13 Frequency dependence during a refraction scenario. The sound speed input is valid across range (top). Normalized intensity at $15 \mathrm{~km}$ range for various frequencies (lower panels). (color online) 
Figure 3.14 Broadband modal energy during a refraction condition is compared to modal decomposition of the PE field. Modal decomposition plots show mode amplitude at $15 \mathrm{~km}$ versus cross-range and frequency. (color online) 135

Figure 3.15 Broadband modal energy during a defocus condition is compared to modal decomposition of the PE field. Modal decomposition plots show mode amplitude at $15 \mathrm{~km}$ versus cross-range and frequency. (color online) 136

Figure 3.16 Broadband modal energy during a focus condition is compared to modal decomposition of the PE field. Modal decomposition plots show mode amplitude at $15 \mathrm{~km}$ versus cross-range and frequency. (color online) 137

Figure 3.17 Energy distribution for a refraction scenario. Broadband energy measurements are shown in the center panels (as point measurements and versus depth). Modal energy distribution is shown on the bottom. (color online) 138

Figure 3.18 Energy distribution for a defocusing scenario. Broadband energy measurements are shown in the center panels (as point measurements and versus depth). Modal energy distribution is shown on the bottom. (color online)

Figure 3.19 Energy distribution for a focusing scenario. Broadband energy measurements are shown in the center panels (as point measurements and versus depth). Modal energy distribution is shown on the bottom. (color online) ............................ 140

Figure 3.20 Close up of mode energy and changes in mode energy during the focus condition shown in Figure 3.19 141

Figure 3.21 Distribution of energy for repeated PE model runs which simulated a NIW traversing the sound field. Actual model data shown by the histogram. These data are best fit to a lognormal distribution. (color online) 142

Figure 3.22 Energy dependence for instances where the acoustic track is nearly parallel to the NIW front. Acoustic angle is varied between $\pm 4^{\circ}$ to show large variability of acoustic receptions for very small angle variations. Left-most panels simulate the xviii 
NIW marching across distance (or time). These data re normalized for each individual plot. Right-most panels show the associated distributions with the expected mean and variance annotated. Mean and variance values are referenced to the entire model data set. NIW sound speed at source provided as a reference. First soliton arrival at each receiving location marked by the asterisks. (color online) 143

Figure 3.23 Modal energy dependence when the acoustic track is $4^{\circ}$ off-axis relative to the NIW marching across the sound field (bottom). I plotted as "point" observations and versus depth to show how modal contributions influence the overall PE field (center panels). (color online) 144

Figure 3.24 Modal energy dependence when the acoustic track is $2^{\circ}$ off-axis relative to the NIW marching across the sound field (bottom). $I_{f}$ plotted as "point" observations and versus depth to show how modal contributions influence the overall PE field (center panels). (color online) 145

Figure 3.25 Modal energy dependence when the acoustic track is parallel to the NIW marching across the sound field (bottom). $I_{f}$ plotted as "point" observations and versus depth to show how modal contributions influence the overall PE field (center panels). (color online) 146

Figure 3.26 Modal energy dependence when the acoustic track is $-2^{\circ}$ off-axis relative to the NIW marching across the sound field (bottom). I plotted as "point" observations and versus depth to show how modal contributions influence the overall PE field (center panels). (color online) 147

Figure 3.27 Modal energy dependence when the acoustic track is $-4^{\circ}$ off-axis relative to the NIW marching across the sound field (bottom). I plotted as "point" 
observations and versus depth to show how modal contributions influence the overall PE field (center panels). (color online)

Figure 3.28 Scintillation index of broadband energy (top) and modal energy (center \& bottom) compared to receiver position relative to the acoustic source (measured in degrees or $\mathrm{km})$. (color online) 149

Figure 3.29 Simulated time arrival data for each bearing angle can be further divided into time-varying fluctuation regimes. Histograms and statistical mean and variance parameters help quantify the differences between dominant physical processes.

\section{Appendix A}

Figure A.1 Event 44 sound speed profile (top). Sound speed at the displaced thermocline (middle). Critical angle, using Snell's law, and measurements from the displaced

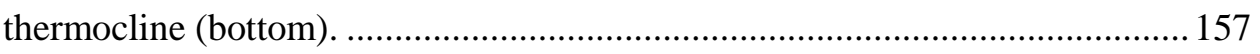

Figure A.2 Mode and frequency dependent critical angles for Event 44......................... 158

Figure A.3 "Back of the envelope" calculation used to estimate maximum time and distance horizontal refraction is likely to take place for Event 44 .............................. 159

Figure A.4 Example chirp sequence for arrivals with low ambient noise levels for hydrophones at the top, middle and bottom of the VLA. This example from the start of the entire Event 44 data set. Entire measured signal shown in grey. Signal that falls within the limits of integration shown in black. Noise before and after each chirp sequence shown in red. $85 \%$ noise threshold shown as a light blue bar. First chirp arrival for each sequence expanded in the right panels.... 160

Figure A.5 Boxplot representing measured ambient at each phone before and after chirp sequence shown in Figure A.4 (top). Noise proves to be normally distributed as shown by histogram and fitted Gaussian PDF (bottom left). Associated CDF with vertical lines corresponding to $75,80,85,90,95$, and $99 \%$ probability. .. 161 
Figure A.6 Example chirp sequence for arrivals with high ambient noise levels for hydrophones at the top, middle and bottom of the VLA. This example taken as the first soliton arrives at the VLA. Entire measured signal shown in grey, vertical scale is identical to Figure A.4. Signal that falls within the limits of integration shown in black. Noise before and after each chirp sequence shown in red. $85 \%$ noise threshold shown as a light blue bar. First chirp arrival for each sequence expanded in the right panels. 162

Figure A.7 Boxplot representing measured ambient at each phone before and after chirp sequence shown in Figure A.6 (top). Noise proves to be normally distributed as shown by histogram and fitted Gaussian PDF (bottom left). Associated CDF with vertical lines corresponding to $75,80,85,90,95$, and $99 \%$ probability. .. 163

Figure A.8 Recalculated energy metrics after a 90\% noise threshold has been implemented. Ramping effect is still evident. 164

Figure A.9 Recalculated energy metrics after an $85 \%$ noise threshold has been implemented. Ramping effect is still evident.

Figure A.10 Stacked time arrival plots for sections of Event 44 data where horizontal refraction may be occurring. 166

Figure A.11 "Point" observations for Event 45, which occurred on 14 Aug 2006 (top). NIW activity at source (center) and at receiving VLA (bottom). This dataset indicative of NIW interference across the entire acoustic track.

Figure A.12 Event 45 "point" observations are plotted versus transmission number (top left), and the data fits well to a lognormal distribution (tip right). Depth dependent variability is plotted using a normalized color scalogram versus transmission number (bottom left), or alternatively shown through a statistical boxplot of received energy upon each hydrophone (bottom right). 168 
Figure A.13 "Point" observations for Event 46, which occurred on 16 Aug 2006 (top). NIW activity at source (center) and at receiving VLA (bottom). This dataset may indicate refraction prior to NIW activity, and also NIW interference. 169

Figure A.14 Event 46 "point" observations are plotted versus transmission number (top left), and the data fits well to a lognormal distribution (tip right). Depth dependent variability is plotted using a normalized color scalogram versus transmission number (bottom left), or alternatively shown through a statistical boxplot of received energy upon each hydrophone (bottom right).

Figure A.15 Stacked time arrival plots for sections of Event 46 data where horizontal refraction may be occurring.

\section{Appendix B}

Figure B.1 Impact upon $I_{f}$ calculations based upon frequency sampling of signals' overall bandwidth. $25 \mathrm{~Hz}$ sampling (left) versus $6.25 \mathrm{~Hz}$ sampling (right)

Figure B.2 Frequency dependent intensity versus cross-range at four arbitrary depths. $25 \mathrm{~Hz}$ sampling (left) fails to capture some of the frequency-dependent structure shown by $6.25 \mathrm{~Hz}$ sampling (right). Figures correspond to those shown in Figure B.1

Figure B.3 Single trace corresponding to cross-range of $-0.2 \mathrm{~km}$, which is the point of maximum refracted energy, previously shown in Figure B.1and Figure B.2. Finer sampling captures spikes and fades that are not apparent in the undersampled profiles. 182

Figure B.4 Various PDFs fit to the ensemble modeled dataset (left). A probability plot helps interpret which distributions are better choices than others 183 
Figure B.5 Depth integrated intensity and modal decomposition of the PE field for modes 15 for a $300 \mathrm{~Hz}$ source. Sound speed input creates a refraction scenario. Source depth changed to $z=45$ meters to better excite Mode 1 . 184

Figure B.6 Depth integrated intensity and modal decomposition of the PE field for modes 15 for a $300 \mathrm{~Hz}$ source. Sound speed input creates a defocus scenario. Source depth changed to $z=45$ meters to better excite Mode 1 . 185

Figure B.7 Depth integrated intensity and modal decomposition of the PE field for modes 15 for a $300 \mathrm{~Hz}$ source. Sound speed input creates a focus scenario. Source depth changed to $z=45$ meters to better excite Mode 1 . 186

\section{Appendix C}

Figure C.1 Modal beamformer weighting. The magnitude of $H_{m}(z, f)$ creates a surface from the frequency dependent modeshapes (top left), corresponding to individual hydrophone weights, one example evaluated at depth $z_{i}$ (bottom left). The angle of $H_{m}(z, f)$ also creates a surface (top right) corresponding to a phase weighting at each hydrophone. A dispersive channel would show curvature, whereas a non-dispersive channel would remain linear (bottom right).

Figure C.2 One-sided amplitude weights for the first five modes, based on modeshapes calculated by the KRAKEN program 195

Figure C.3 Expected wavenumbers and group velocities, used to potentially calculate modal dispersion within the shallow water waveguide 196

Figure C.4 Expected mode arrivals based on dispersion for the first five modes. Original transmission shown at far left, expected arrival shown at far right. 197 


\section{MANUSCRIPT - I}

Intended for submission to the Journal of the Acoustical Society of America

\section{Evidence of escalating acoustic intensity preceding a strong internal wave event during the Shallow Water' 06 experiment}

\section{Corresponding Author:}

\section{Georges A. Dossot}

Department of Ocean Engineering, University of Rhode Island, Narragansett, Rhode Island 02882, georges.dossot@gmail.com

\section{Contributing Authors:}

\section{Mohsen Badiey}

College of Marine and Earth Studies, University of Delaware, Newark, Delaware 19716

\section{Kevin B. Smith}

Department of Physics, Naval Postgraduate School, Monterey, California 93943

\section{James H. Miller, Gopu R. Potty}

Department of Ocean Engineering, University of Rhode Island, Narragansett, Rhode Island 02882

\section{James F. Lynch}

Department of Applied Ocean Physics and Engineering, Woods Hole Oceanographic Institution, Woods Hole, Massachusetts 02543 
Abstract: During the Shallow Water '06 (SW06) experiment, a J-15 acoustic source deployed from the Research Vessel Sharp transmitted broadband (50-450 Hz) chirp signals $15 \mathrm{~km}$ away from a vertical line array. The array was intentionally positioned near the shelf-break front and in an area where internal waves are known to occur.

During the same time an internal wave, labeled "Event 44," passed through the sound field such that the internal wave front was nearly parallel to the acoustic transmission path. Measured data shows substantial intensity fluctuations that vary over time and space due to complex multimode and multipath (both two- and three-dimensional) interference patterns. Of specific interest are fluctuations of measured intensity preceding the internal wave's arrival. Acoustic data are compared to simultaneous oceanographic measurements from ship-borne and moored environmental sensors. Oceanographic measurements during the internal wave event are used to replicate the sound speed profile, and implemented in the three-dimensional Monterey-Miami Parabolic Equation (MMPE) code. Both acoustic measurements and simulations show escalating acoustic intensity prior to the internal wave's arrival due to horizontal refraction effects.

(C) 2011 Acoustical Society of America

PACS numbers: 43.30.Es, 43.30.Re, 43.30.Dr, 43.30.Zk 


\subsection{INTRODUCTION AND BACKGROUND}

Strong nonlinear internal waves (NIWs) are well-known to occur in shallow water, usually near the continental shelf break, due to interactions with tidal forcing and the non-uniform ocean bathymetry. Since the early 1990s, it has been known that these NIWs have a profound effect upon acoustic propagation, which was one of the primary goals for the Shallow Water '06 experiment (SW06). ${ }^{1}$ The purpose of this article is to highlight a specific data set from the SW06 experiment, which shows evidence of horizontal refraction of acoustic signals due to an approaching NIW. In this instance, the source-receiver path was nearly parallel to the propagating NIW fronts, and acoustic intensity receptions steadily escalated prior to the NIW's arrival. To shed insight into the acoustic data, a measured oceanographic sound speed profile was employed as the primary environmental input to a three-dimensional parabolic equation model. These model results offer conceptual visualization of horizontal refraction effects and also show corresponding escalation, or "ramping," of acoustic intensity prior to the NIW's arrival.

Previous experiments and reports have identified acoustic intensity fluctuations varying between 3-20 dB due to interactions with the acoustic field and NIWs.

Specifically, Badiey, et al. ${ }^{2}$ identified acoustic propagation regimes based upon angle dependence of an internal wave front relative to the source-receiver path, $\alpha$. When the source-receiver path is nearly perpendicular to a NIW, mode coupling dominates. At angles closer to $45^{\circ}$, adiabatic propagation dominates because neither mode coupling nor horizontal refraction effects play a strong role. Finally, at low angles, horizontal refraction and focusing dominate. Theoretical predictions of horizontal refraction effects were previously offered by Katsnelson and Pereselkov. ${ }^{3}$ Shortly thereafter, Badiey, et 
$a l .{ }^{4}$ documented horizontal regime fluctuations between 6-7 dB derived from SWARM-

$95^{5}$ data, and more recently fluctuations of $7 \mathrm{~dB}$ in SW06 data have been recognized by Luo, et al. ${ }^{6}$ The data highlighted in this article demonstrate similar intensity fluctuations from an internal wave event experienced aboard the University of Delaware's Research Vessel (R/V) Sharp during the SW06 experiment ${ }^{7}$ due to horizontal refraction effects.

\subsection{EXPERIMENTAL SETUP}

The SW06 experiment took place during the summer of 2006 off the coast of New Jersey with a primary goal of further understanding acoustic propagation in coastal waters. The multi-institutional endeavor was sponsored by the Office of Naval Research, and included numerous research vessels, scientific moorings, and principal investigators. The experimental location was proximate to the continental shelf break front, near the site of previous acoustic studies, ${ }^{5}$ and was ideally situated in an environment where internal waves are known to occur. During SW06, the R/V Sharp was deployed for a three-week period (1-21 Aug) and experienced over 50 internal wave events.

Figure 1.1 depicts the portion of SW06 experimental area relevant to internal wave "Event 44." The map in this figure shows the primary acoustic receiver, environmental moorings, and acoustic source locations- including the R/V Sharp's J-15 source. The primary receiver, the WHOI "Shark" 16-element vertical line array (VLA), is situated at the vertex of the acoustic transmission paths. During periods when the moored SW06 sources were not transmitting, the R/V Sharp transmitted a series of repeating signals every three minutes, including 23 broadband chirps $(50-450 \mathrm{~Hz})$ at 74 meters depth. At roughly 3:30 AM (GMT) on August 14th, the surface expression of a large slow-moving 
internal wave train appeared on the ship's RADAR. Overlaid on the map is one snapshot of RADAR imagery as Event 44 first reached the ship and acoustic source.

\subsection{OCEANOGRAPHIC MEASUREMENTS AND INFERENCES}

Approximations from the RADAR imagery show that the leading edge of the soliton train propagated at roughly $0.5 \mathrm{~m} / \mathrm{s}$, with little evidence of curvature (although often known to occur ${ }^{8}$ ). RADAR also showed the NIW train propagated northwest by west at a bearing of 308 degrees for five hours, evident between 4:00 and 9:00 GMT.

Environmental water column measurements confirm a strong internal wave event during this time. The bottom panels of Figure 1.2 exhibit internal wave activity at the R/V Sharp's J-15 source, at the WHOI VLA receiver, and at an environmental mooring midway between the source and receiver. The R/V Sharp's downward looking Acoustic Doppler Current Profiler (ADCP) recorded evidence of a strongly oscillating pycnocline as the internal wave passed beneath the ship. Environmental mooring SW32, although 844 meters off-axis from the source-receiver path, also shows very strong internal wave activity at a midway point between transmission and reception. Finally, interpolated sound-speed values at the WHOI VLA exhibit internal wave activity during the same time period. Comparing internal wave activity at the source and receiver, the environmental data indicates the NIW arrived at the receiver location about one hour prior to the acoustic source location. Assuming a speed of $0.5 \mathrm{~m} / \mathrm{s}$ (estimated by RADAR imagery) we approximate a bearing difference $\alpha$, of approximately 5-6 degrees between the propagating internal wave front and the source-receiver path. This geometry results in a 15 kilometer long source-receiver path, with a nearly parallel, slow-moving, and straight-line internal wave front reaching the acoustic receiver roughly $1.5 \mathrm{~km}$ before 
the acoustic source. Given these environmental and experimental conditions, we have an ideal scenario for acoustic horizontal refraction due to the approaching NIW.

\subsection{ACOUSTIC MEASUREMENTS AND EVIDENCE OF HORIZONTAL REFRACTION}

One way to visualize the role of the internal wave's presence on acoustic transmissions is to examine the intensity fluctuations received at the VLA, and correlate them with NIW activity. The top panel of Figure 1.2 shows integrated energy "point" statistics $^{9}$ of each signal received along the height of the VLA. The signals are high-pass filtered (100 Hz cutoff frequency) to remove broadband noise, and match-filtered to extract the transmitted chirp signal. Temporally integrated energy intensity, $I_{\tau}$, of each signal $k$, and at depth $z$, is calculated by integrating the match- and high-pass filtered intensity $I$, over the energetic duration of the signal $\tau$, as shown in equation (1.1). These data are then normalized such that the mean value is one; $\left\langle I_{\tau}\right\rangle=1$. This metric provides visual interpretation of the peak energy variability, and variability of energy distribution throughout the water column. Normalizing data in this manner offers good visualization of fluctuating signal intensifications $I_{\tau}>1$; however interpreting signal fades $\left(0<I_{\tau}<1\right)$ can be more difficult to discern. The purpose of this analysis is to show evidence of escalating intensity, therefore it is a suitable technique.

$$
I_{\tau}(z, k)=\int I(\tau, z, k) d \tau
$$

Comparing the point statistics to the internal wave activity in Figure 1.2 and in Multimedia file 1(Movie, available at JASA online), some prominent features are 
visually striking. Firstly, the "refraction" regime, between 3:00 and 5:40 GMT, shows escalating peak intensity values, inferring strong refraction, and constructive interference in the horizontal acoustic plane. Before 4:30, the NIW has neither reached the source nor receiver, and a "ramping" of energy that is well distributed along the VLA height is apparent. Between 4:30 and 5:40, the peak intensity continues to escalate, while the overall received energy along the VLA is somewhat suppressed. The suppression may be due to defocusing and interference effects as the internal wave starts to cross into the source-receiver path. At 5:40, the "focusing" regime, a strong collection of high-energy signals occurs just as Event 44 arrives at the $\mathrm{J}-15$ source. This final stage of energy ramping over time is possibly due to trapped acoustic energy between solitons in the internal wave train (identified by a white dotted line on the ADCP echo, Figure 1.2). Movie 1 (available on JASA online) shows radar imagery over time, indicating this focusing event likely occurs as the first two or three solitons within the NIW train reach the acoustic source. The NIW interference regime occurs during internal wave activity over the entire source-receiver path. It can be described by an immediate quelling of the received energy with irregular fluctuations throughout the NIW's presence. After the NIW has arrived at the acoustic source, the acoustic field is dominated by a wavering intensity pattern caused by complicated constructive and destructive interference from focusing and de-focusing effects.

\subsection{THREE-DIMENSIONAL PARABOLIC EQUATION MODELING}

Because other acoustic sources transmitted during the experimental timeframe, the R/V Sharp's J-15 could not transmit on a persistent basis. To better understand the intermittent acoustic data, the measured oceanographic sound speed profile at a midway 
point between the source and receiver (environmental mooring SW32) was employed as the primary environmental input to a three-dimensional, Cartesian coordinate version of the Monterey-Miami Parabolic Equation (MMPE) model. ${ }^{10,11,12,13}$ For this model, we assume the internal wave to be consistent across range in a straight-line approximation, although others have shown curvature to be an important factor when it exists. ${ }^{8,14}$ The MMPE model simulated a 5 by 15 kilometer ocean region in cross-range and range, respectively. Ocean bottom parameters were simplified because the focus of this work is to measure impacts due to water column variability. The bathymetry was modeled as a flat and constant 80 meters depth. A two layered sediment interface of the New Jersey shelf defined by Jiang, et al. ${ }^{15}$ simulated the sediment parameters, excluding the effects of shear. The acoustic source was modeled as a wide-angle $300 \mathrm{~Hz}$ source to approximate propagation at the center frequency of the broadband chirp signals used in the actual experiment. One hundred model runs were performed; each one representing a further advancing straight-line NIW train marching towards the source-receiver path.

Three different model run iterations are shown in Figure 1.3, highlighting refraction, defocusing, and focusing effects in the horizontal plane. The top left quadrant (MMPE run 70) shows the measured sound speed profile versus depth and cross-range implemented in the MMPE model. To create this sound speed profile, mooring measurements on SW32 captured versus time were converted to distance (cross-range) assuming a constant NIW speed of $0.5 \mathrm{~m} / \mathrm{s}$. Immediately to the right of this sound speed input is a plot of the acoustic intensity, integrated over the depth of the modeled ocean volume. The acoustic source, marked by a star, is located at zero range and cross-range, and the plots are scaled in decibels relative to a unity source level at one meter. This 
specific model run shows strong evidence of horizontal refraction, akin to a Lloyd's mirror, suggested by Lynch, et al. ${ }^{16}$ and recently verified by Badiey, et al. ${ }^{17,18}$, where energy is refracted off the internal wave front, creating constructive interference in regions ahead of the NIW. The top right quadrant (MMPE run 74) simulates the internal wave as the leading soliton first hits the acoustic source. Here, defocusing, or "antiducting," causes spreading of the acoustic energy. The bottom left quadrant (MMPE run 78) simulates the source being trapped by two solitons within the NIW, causing strong focusing, or "ducting."

To simulate Event 44, we assume the VLA was situated five degrees off-parallel, within a one degree tolerance $\left(-5.5^{\circ} \leq \alpha \leq-4.5^{\circ}\right)$. The integrated intensity plots in Figure 1.3 show a white star at $15 \mathrm{~km}$ range, and five degrees off-axis, representing the VLA positioned such that the NIW reaches the receiving elements prior to the acoustic source. The bottom right quadrant in Figure 1.3 shows "point" receptions of the simulated VLA positioned between 4.5 and 5.5 degrees off-axis. Intensity of the PE field, $I_{P E}$, for each depth $z$, each model run (or simulated acoustic transmission), $k$, and over the angular bounds, $\alpha$, is normalized such that the mean intensity is one; $\left\langle I_{P E}=1\right\rangle$. These point statistics represent separate MMPE model runs (or acoustic transmissions) as the NIW marches forward over time and space. Even very small angular changes cause strong variations in the model data; therefore the aggregate point receptions flanked by these angular bounds provide better visual interpretation of the results. Comparing the measured and modeled point observations (Figure 1.2 and Figure 1.3, respectively), several similar trends exist. Foremost, the fluctuations between the measured and modeled data show the same general structure and scale. Both data sets start with an 
initially low level of acoustic variability, where the NIW has little influence, even in the horizontal plane. This can be termed the "quiescent" regime ${ }^{19}$ because the sound speed profile is relatively uniform over the entire ocean volume. Escalating intensity receptions, or "ramping" prior to the NIW's arrival, appear as the soliton train approaches the acoustic source. This period corresponds to the refraction regimes identified in Figure 1.2 and Figure 1.3. In both the modeled and measured data, the ramping ends in a pronounced, short duration interval of high-energy receptions. A few possible scenarios exist for this high energy period, which was hypothesized as possible focusing in the measured data. While focusing could be the prominent cause of this spike in energy, the VLA was situated sufficiently off-axis, such that very strong focusing from energy trapped between solitons (shown in MMPE run 78) will not likely occur. For focusing to play a prominent role, range dependence in the NIW's structure, such as a truncated duct ${ }^{8}$ could cause trapped energy to propagate for a portion of the acoustic path, spreading energy towards the vicinity of the VLA. However, the model and experimental geometry suggests that defocusing at the acoustic source may be causing the energy spike received at the VLA. Defocusing (shown in MMPE run 74) spreads acoustic energy in the horizontal plane, pointing energy off-axis and towards the VLA position. Following this energy defocusing event due to the first soliton arrival, focusing (shown in MMPE run 78) points energy away from the VLA, causing a suppression of received energy. Lastly, as the NIW crosses the entire source-receiver path, substantially fluctuating intensity values are marked by a NIW interference regime evident in both the measured and model data. 
Directly below the point receptions shown in Figure 1.3, is a color-scale plot of the peak intensity at each depth, between the range of 4.5 and 5.5 degrees off-axis, $I_{P E, P e a k}(z, k, \alpha) ;$ where,

$$
I_{P E, \text { Peak }}(z, k, \alpha)=\max \left(\left\langle\left. I_{P E}(z, k)\right|_{\alpha=-5.5^{\circ} \rightarrow-4.5^{\circ}}\right\rangle\right)
$$

The depth dependent intensity variability shown in this color-scale plot infers possible modal dependency within the refraction and NIW interference regimes. In the refraction regime, steadily escalating energy in an acoustic channel centered at 20 meters indicates the possibility of a dominant mode. As the NIW reaches (and crosses into) the sourcereceiver path, energy fluctuates greatly with depth, indicating a very complicated interference regime where different modes may be excited based upon the location of the NIW relative to the source and receiver.

\subsection{CONCLUSION}

In this article we highlight a ramping phenomenon associated with escalating acoustic intensity preceding an internal wave's arrival due to horizontal refraction effects.

Although much can be interpreted from the initial data analysis and modeling presented here, future work will provide a better understanding of the underlying physics relevant to these data, and also the more general aspect of acoustic propagation in regions with NIW activity. The refraction, focusing, and defocusing that may be occurring in the measured data warrant further investigation - to include examining modal refraction and coupling. Additionally, examining complicating oceanographic factors, such as non- 
uniformity in the internal wave structure and the presence of a thermohaline bottom intrusion, may show other dominant features causing these intensity fluctuations. 


\section{ACKNOWLEDGEMENTS}

The authors are indebted to the dedicated and hard-working participants of the SW06 experiment, with special thanks to the WHOI mooring crews and personnel aboard the R/V Sharp. Our deepest gratitude is extended to ONR who funded the SW06 experiment, and the exciting research that has followed. 


\section{REFERENCES AND LINKS}

1. A. E. Newhall, T. F. Duda, K. Von Der Heydt, J. D. Irish, J. N. Kemp, S. A. Lerner, S. P. Liberatore, Y. T. Lin, J. F. Lynch, A. R. Maffei and others, "Acoustic and oceanographic observations and configuration information for the WHOI moorings from the SW06 experiment," WHOI technical report No. WHOI-2007-04 (2007).

2. M. Badiey, B. G. Katsnelson, J. F. Lynch and S. Pereselkov, "Frequency dependence and intensity fluctuations due to shallow water internal waves," J. Acoust. Soc. Am. 122 (2), 747-760 (2007).

3. B. G. Katsnelson and S. A. Pereselkov, "Low-Frequency Horizontal Acoustic Refraction Caused by Internal Wave Solitons in a Shallow Sea.," Acoust. Phys. 46 (6), 684 (2000).

4. M. Badiey, Y. Mu, J. Lynch, J. Apel and S. Wolf, "Temporal and azimuthal dependence of sound propagation in shallow water with internal waves," IEEE J. Ocean. Eng. 27 (1), 117-129 (2002).

5. J. R. Apel, M. Badiey, C.-S. Chiu, S. Finette, R. Headrick, J. Kemp, J. F. Lynch, A. Newhall, M. H. Orr, B. H. Pasewark, D. Tielbuerger, A. Turgut, K. Von Der Heydt and S. Wolf, "An overview of the 1995 SWARM shallow-water internal wave acoustic scattering experiment," IEEE J. Ocean. Eng. 22 (3), 465-500 (1997).

6. J. Luo, M. Badiey, E. A. Karjadi, B. Katsnelson, A. Tskhoidze, J. F. Lynch and J. N. Moum, "Observation of sound focusing and defocusing due to propagating nonlinear internal waves," J. Acoust. Soc. Am. 124 (3), 66 (2008).

7. M. Badiey, B. Katsnelson, J. Luo, L. Brown, J. Yang, G. A. Dossot, S. E. Crocker, J. Seigel, J. Largeaud and L. Wan, "R/V Sharp SW06 Cruise Technical Report," University of Delaware Technical Report (2010).

8. J. F. Lynch, Y. T. Lin, T. F. Duda and A. E. Newhall, "Acoustic Ducting, Reflection, Refraction, and Dispersion by Curved Nonlinear Internal Waves in Shallow Water," IEEE J. Ocean. Eng. 35 (1), 12-27 (2010). 
9. A. Fredricks, J. A. Colosi, J. F. Lynch, G. Gawarkiewicz, C.-S. Chiu and P. Abbot, "Analysis of multipath scintillations from long range acoustic transmissions on the New England continental slope and shelf," J. Acoust. Soc. Am. 117 (3), 1038-1057 (2005).

10. K. B. Smith and J. A. Colosi, "Effects of solitons on acoustic energy flow in three dimensions," J. Acoust Soc. Am. 124 (66), 1038-1057 (2008).

11. K. B. Smith, C. W. Miller, A. F. D'Agostino, B. Sperry, J. H. Miller and G. R. Potty, "Three-dimensional propagation effects near the mid-Atlantic Bight shelf break (L)," J. Acoust. Soc. Am. 112 (2), 373-376 (2002).

12. K. B. Smith and F. D. Tappert, "Horizontal refraction and the uncoupled azimuth approximation," 343 (2004).

13. K. B. Smith, "Convergence, Stability, and Variability of Shallow Water Acoustic Predictions Using a Split-Step Fourier Parabolic Equation Model.," Journal of Computational Acoustics 9 (1), 243 (2001).

14. L. Jing and M. Badiey, "Frequency dependent beating patterns and amplitude increase during an approaching internal wave," J. Acoust. Soc. Am. (Submitted).

15. Y.-M. Jiang, N. R. Chapman and M. Badiey, "Quantifying the uncertainty of geoacoustic parameter estimates for the New Jersey shelf by inverting air gun data," J. Acoust. Soc. Am. 121 (4), 1879-1894 (2007).

16. J. F. Lynch, J. A. Colosi, G. G. Gawarkiewicz, T. F. Duda, A. D. Pierce, M. Badiey, B. G. Katsnelson, J. E. Miller, W. Siegmann, C.-S. Chiu and A. Newhall, "Consideration of fine-scale coastal oceanography and 3-D acoustics effects for the ESME sound exposure model," IEEE J. Ocean. Eng. 31 (1), 33-48 (2006).

17. M. Badiey, J. F. Lynch, Y. T. Lin and B. G. Katsnelson, "Horizontal reflection of a low-frequency sound signal from a moving nonlinear internal wave front," J. Acoust. Soc. Am. 127 (3), 1974-1974 (2010).

18. M. Badiey, B. G. Katsnelson, Y. T. Lin and J. F. Lynch, "Acoustic multipath arrivals in the horizontal plane due to approaching nonlinear internal waves," J. Acoust. Soc. Am. 129 (4) (2011). 
19. S. Finette, M. H. Orr, A. Turgut, J. R. Apel, M. Badiey, C.-s. Chiu, R. H. Headrick, J. N. Kemp, J. F. Lynch, A. E. Newhall, K. v. Heydt, B. Pasewark, S. N. Wolf and D. Tielbuerger, "Acoustic field variability induced by time evolving internal wave fields," J. Acoust. Soc. Am. 108 (3), 957-972 (2000). 


\section{FIGURES}

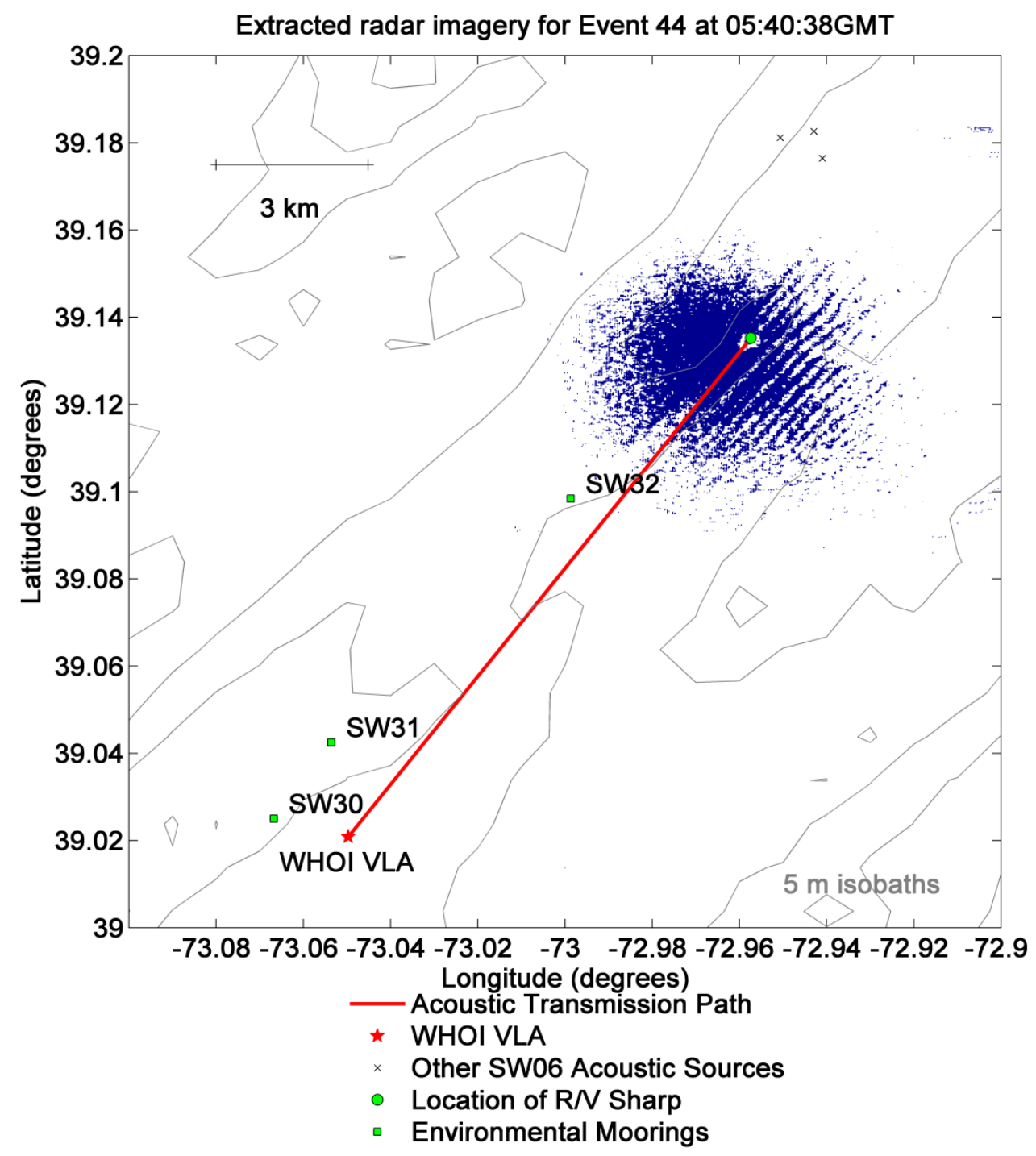

Figure 1.1 Close-up of SW06 experimental area and overlaid ship RADAR image. A strong internal wave event propagated nearly parallel to the source (R/V Sharp) and receiver (WHOI VLA) acoustic track. (color online) 

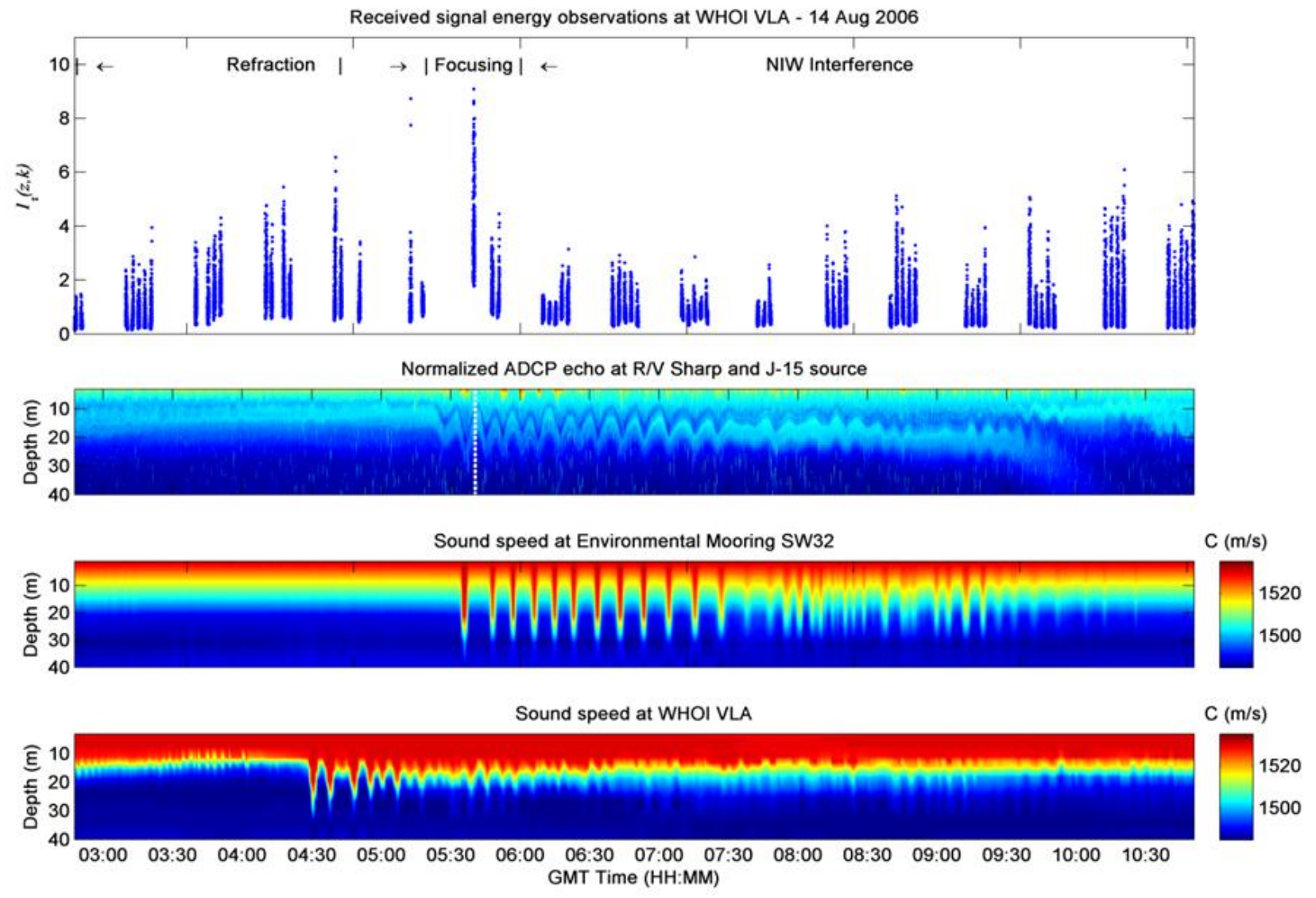

Figure 1.2 Acoustic data compared to environmental data. (Top) Measured normalized energy levels received upon each hydrophone of the WHOI VLA versus time. Refraction occurs prior to the NIW's arrival, with possible focusing as the NIW arrives at the acoustic source. A complicated NIW interference regime follows; (Bottom 3 plots) Environmental sensors including R/V Sharp ADCP (at the acoustic source) showing NIW pycnocline, mooring SW32 (midway between sourcereceiver), and interpolated sound speed at the WHOI receiving VLA, all exhibiting strong internal wave activity. (color online) 

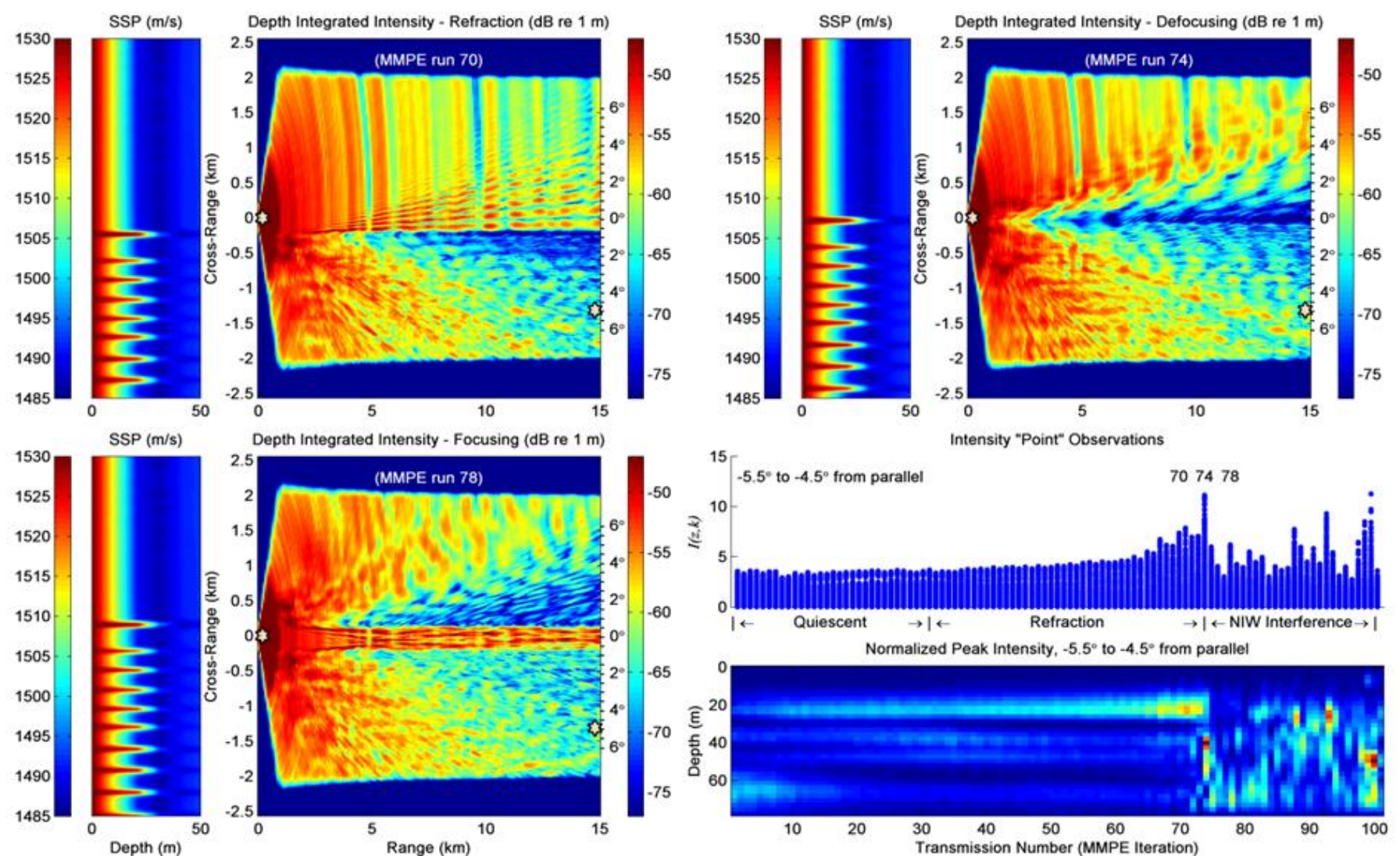

Figure 1.3 Measured sound speed profile versus depth used for PE simulation (Top left quadrant). Sound speed varies with depth and cross-range, but assumed valid versus range in a "straight-line" approximation. Depth-integrated intensity is shown to the right of the sound speed profile. In this instance, the NIW has not arrived at the source and refraction dominates; (Top right quadrant) NIW has arrived at the source and defocusing dominates; (Bottom left quadrant) NIW traps the source between two solitons and focusing dominates; (Bottom right quadrant) The NIW sound speed profile advances over 100 MMPE simulations to simulate the actual data. Normalized received intensity between 4.5 to 5.5 degrees from the propagating NIW and source-receiver path show escalating intensity prior to the NIW's arrival in the "Refraction" regime. (color online) 


\section{Measured intensity fluctuations due to horizontal}

\section{refraction during internal wave activity in the Shallow}

\section{Water '06 experiment}

\section{Corresponding Author:}

\section{Georges A. Dossot}

Department of Ocean Engineering, University of Rhode Island, Narragansett, Rhode Island 02882, georges.dossot@gmail.com

\section{Contributing Authors:}

\section{Mohsen Badiey}

College of Marine and Earth Studies, University of Delaware, Newark, Delaware 19716

\section{Kevin B. Smith}

Department of Physics, Naval Postgraduate School, Monterey, California 93943

\section{James H. Miller, Gopu R. Potty}

Department of Ocean Engineering, University of Rhode Island, Narragansett, Rhode Island 02882

\section{James F. Lynch}

Department of Applied Ocean Physics and Engineering, Woods Hole Oceanographic Institution, Woods Hole, Massachusetts 02543 


\begin{abstract}
Measurements from the Shallow Water '06 experiment show time-varying modulation of the acoustic field due to nonlinear internal wave (NIW) activity. These measurements occurred during instances where the acoustic track was nearly parallel to the approaching NIW, such that horizontal refraction effects are important to consider. A brief background of the oceanography specific to the experimental area is provided, and local oceanographic data is compared to acoustic data. Using a statistical analysis of the experimental data, time dependence of the sound field as the NIW traverses across the acoustic track is shown. Fluctuation regimes where the dominant physical processes for each portion of the measured data are suggested. Broadband intensity metrics provide a global perspective of the time-varying acoustic field due to NIW activity. Modal decomposition of the acoustic data lends further insight into the governing physical processes at work. Three-dimensional normal mode modeling qualitatively shows the influence of NIW modulations on the acoustic field.
\end{abstract}

(C) 2011 Acoustical Society of America

PACS numbers: 43.30.Es, 43.30.Re, 43.30.Dr, 43.30.Zk 


\subsection{INTRODUCTION AND BACKGROUND}

The confluence of strong nonlinear internal waves (NIWs) with an underwater soundfield has become an increasingly well understood recipe for water-column induced acoustic variability. Research in this area can be traced back to the World War II era, when acoustic regions of high intensity and shadow zones were predicted using internal wave data from offshore California. ${ }^{1}$ This work was later revisited in the early sixties, where intensity contrasts up to $22 \mathrm{~dB}$ were predicted via ray theory when a series of internal waves existed in the media. ${ }^{2}$ For many years afterwards, effects of internal waves were considered to be important factors in long-range and low-frequency propagation, contributing to stochastic multipath effects. In these cases, a statistical approach is well-suited to interpret multipath interference properties of the acoustic field, ${ }^{3,4,5}$ including water column variability due to the linear internal wave spectrum. ${ }^{6}$ Acoustic variability due to the shallow water NIW field was first examined closely in the early 1990s, when an experiment in the Yellow Sea showed that NIWs can cause substantial intensity fluctuations of low-frequency signals (up to $25 \mathrm{~dB}$ ). ${ }^{7}$ This experiment, and other developing questions, ${ }^{8}$ served as the motivation for further investigations tailored to explore the effects of water column inhomogeneity in the sound field. Since then, large-scale ocean experiments such as PRIMER, ${ }^{9} \mathrm{SWARM},{ }^{10}$ ASIAEX, ${ }^{11,12}$ and more recently the Shallow Water '06 (SW06) experiment, ${ }^{13}$ have furthered our knowledge of the fluctuating sound field in the shallow water environment.

These recent endeavors have greatly increased our insight into this area of research. Specifically relevant to this paper, at low frequencies (between $50 \mathrm{~Hz}$ and $500 \mathrm{~Hz}$ ), the relative angle between the source-receiver path and an approaching NIW governs the 
acoustic propagation processes that dominate the fluctuating sound field. This azimuthal dependence defined by Badiey, et al. ${ }^{14}$ has roots in geoacoustic studies, where acoustic dispersion was shown to vary with angle when a non-uniform bottom structure is present. ${ }^{15}$ This same reasoning was applied to NIWs, delineating acoustic regimes where mode coupling, adiabatic propagation, or horizontal refraction dominates. In the perpendicular case, when the acoustic track crosses through the NIW, mode coupling dominates. ${ }^{16}$ During instances where the acoustic track is neither parallel nor perpendicular to the NIW field, adiabatic propagation governs. Alternatively, when the NIW is parallel to the acoustic track, horizontal refraction and focusing dominate, and the "horizontal-rays and vertical modes" approach is well-suited. ${ }^{17,18}$ Horizontal refraction effects due to NIW activity, formerly predicted through modeling ${ }^{19}$ and theory, ${ }^{20}$ were first observed by Badiey, et al. ${ }^{21}$ during the SWARM experiment, where ducting of low frequency $(20-300 \mathrm{~Hz}$ ) signals increased the sound field between 6-10 dB. Following these early results, several numerical, theoretical, and experimental studies ${ }^{22-29}$ have solidified our understanding of these effects.

It is the purpose of this paper to complement existing work, and offer new evidence of horizontal refraction using experimental data from the SW06 experiment. Two scenarios are offered where a NIW crest crossed the acoustic track in a nearly parallel fashion, demonstrating evidence of horizontal refraction, focusing, and defocusing phenomena. Using a statistical analysis of the experimental data, the time dependence of the acoustic field as the NIW traverses the acoustic track is shown. Modal dependence of this time varying process is also explored, and ultimately, fluctuation regimes where the dominant physical processes for each portion of the measured data are suggested. 
This paper is organized in the following manner. A general, time-varying scenario is defined where the acoustic track is nearly parallel to an approaching NIW train, and intensity fluctuations regimes are defined. Following this general case, two specific investigations from the SW06 experiment are summarized. A brief background of the oceanography specific to that region is provided, and the local oceanographic data is compared to acoustic data. Broadband energy metrics are calculated to give a global perspective of the time-varying acoustic field. Following this, broadband modal decomposition lends further insight into these fluctuations, and three-dimensional modeling qualitatively shows mode-dependent horizontal refraction scenarios. Lastly, a suggested interpretation of the observational data is provided for consideration.

\subsection{INTENSITY FLUCTUATION REGIMES FOR THE PARALLEL CASE}

The previously mentioned studies have shown that propagating NIWs strongly influence the temporal variability of low-frequency acoustic signals at very short time scales - on the order of minutes. Typical acoustic fluctuations due to sound field modulation from NIWs are trademarked by signal intensification and by deep fades. These types of fluctuations are especially evident in the case where the acoustic track is parallel to the propagating NIW front - and where refraction, focusing and defocusing effects dominate.

In order to better understand these three dominating conditions, let us consider the three-dimensional Helmholtz equation that defines the complex pressure field for space and time, $P(x, y, z, t)=p(x, y, z) e^{-j \omega t}$, which, up to a scaling factor for the source level, is given by 


$$
\rho \nabla \cdot\left(\frac{1}{\rho} \nabla p\right)+\frac{\omega^{2}}{c^{2}(x, y, z)} p=\delta\left(x-x_{s}\right) \delta\left(y-y_{s}\right) \delta\left(z-z_{s}\right)
$$

where $\rho$ is the medium density, $\omega=2 \pi f$ is the acoustic frequency with $f$ measured in Hertz, and $c(x, y, z)$ is the acoustic sound speed at a point in three dimensional Cartesian space. The sound speed may be complex, where the imaginary portion accounts for absorption. The right hand side specifies a source at location $\left(x_{s}, y_{s}, z_{s}\right)$ using the properties of the Dirac-delta function $\delta$. If we reference our source at the origin in the $x, y$ plane $\left(x_{s}=y_{s}=0\right)$, and we expand (2.1), it becomes

$$
\begin{aligned}
& \rho \frac{\partial}{\partial x}\left(\frac{1}{\rho} \frac{\partial p}{\partial x}\right)+\rho \frac{\partial}{\partial y}\left(\frac{1}{\rho} \frac{\partial p}{\partial y}\right)+\rho \frac{\partial}{\partial z}\left(\frac{1}{\rho} \frac{\partial p}{\partial z}\right) \\
& +\frac{\omega^{2}}{c^{2}(x, y, z)} p=-\delta(x) \delta(y) \delta\left(z-z_{s}\right)
\end{aligned}
$$

We seek a solution pertinent to modal decomposition in the form

$$
p(x, y, z)=\sum_{m} \Phi_{m}(x, y) \Psi_{m}(z \mid(x, y)),
$$

where $\Phi_{m}(x, y)$ is the complex modal amplitude, and $\Psi_{m}(z \mid(x, y))$ is the vertical mode shape, both evaluated at position $(x, y)$ for mode $m$. Additionally, let us neglect any density differences along the $x, y$ plane, and assume that density is strictly a function of depth, $\rho=\rho(z)$. This form allows us to calculate the "local modes" at each point in the horizontal plane. We enforce boundary conditions for this problem, 


$$
\begin{aligned}
& \rho(z) \frac{d}{d z}\left(\frac{1}{\rho(z)} \frac{d}{d z} \Psi_{m}(z \mid(x, y))\right) \\
& +\left(\frac{\omega^{2}}{c^{2}(x, y, z)}-k_{r m}^{2}(x, y)\right) \Psi_{m}(z \mid(x, y))=0
\end{aligned}
$$

such that a pressure release is imposed at the surface, and for any layered sediments, we impose continuity of normal velocity at each interface. Here, $k_{r m}(x, y)$ is the horizontal wavenumber at each local point $x, y$, and governs the effective index of refraction, determined by ${ }^{30}$

$$
n_{m}(x, y)=\frac{k_{r m}(x, y)}{k_{r m 0}}=\frac{v_{m 0}}{v_{m}(x, y)}
$$

where $v_{m}$ is the phase velocity of each mode; $k_{m 0}$ and $v_{m 0}$ are reference values, chosen at an arbitrary location (such as the origin), based upon assumption, or possibly a mean value over some unperturbed area. Therefore, changes in the water column due to NIWs will cause perturbations to the mode-dependent horizontal wavenumbers (or phase velocities). These perturbations are small values, quite close to the reference values, and much less than the difference between modal eigenvalues. The difference $\Delta k_{r m}$, between a perturbed condition $k_{r m}^{\prime}$, and the unperturbed condition $k_{r m 0}$, satisfies

$$
\begin{aligned}
& \Delta k_{r m}=\left|k_{r m 0}-k_{r m}^{\prime}(x, y)\right| \ll k_{r m 0} \\
& \Delta k_{r m} \ll\left|k_{r m 0}-k_{r n 0}\right| ; \quad n=m \pm 1 .
\end{aligned}
$$

If we substitute our proposed solution into the three-dimensional Helmholtz equation, and also apply the operator 


$$
\int(\cdot) \frac{\Psi_{n}(x, y, z)}{\rho} d z
$$

we arrive at

$$
\begin{aligned}
& \frac{\partial^{2} \Phi_{n}}{\partial x^{2}}+\frac{\partial^{2} \Phi_{n}}{\partial y^{2}}+k_{r m}^{2}(x, y) \Phi_{n}+\sum_{m} A_{m n} \Phi_{m} \\
& +\sum_{m} B_{m n} \frac{\partial \Phi_{m}}{\partial x}+\sum_{m} 2 C_{m n} \frac{\partial \Phi_{m}}{\partial y}=-\delta(x) \delta(y) \delta\left(z-z_{s}\right) .
\end{aligned}
$$

Here, $A_{m n}, B_{m n}, C_{m n}$ are mode-coupling matrices given by

$$
\begin{aligned}
& A_{m n}=\int\left(\frac{\partial^{2}}{\partial x^{2}}+\frac{\partial^{2}}{\partial y^{2}}\right) \Psi_{m} \frac{\Psi_{n}}{\rho} d z ; \\
& B_{m n}=-B_{m n}=\int \frac{\partial \Psi_{m}}{\partial x} \frac{\Psi_{n}}{\rho} d z ; \\
& C_{m n}=-C_{m n}=\int \frac{\partial \Psi_{m}}{\partial y} \frac{\Psi_{n}}{\rho} d z .
\end{aligned}
$$

Because we are primarily dealing with small angles between the acoustic track and the propagating NIW, we can assume adiabatic propagation dominates, thereby simplifying our problem. Disregarding mode coupling, and reverting to our original modal index $m$, we have

$$
\frac{\partial^{2} \Phi_{m}}{\partial x^{2}}+\frac{\partial^{2} \Phi_{m}}{\partial y^{2}}+k_{r m}^{2}(x, y) \Phi_{m}=-\Psi_{m}\left(z_{s}\right) \delta(x) \delta(y) .
$$

Our result is now strictly dependent on a horizontal coordinate system. This is referred to as the horizontal refraction equation and specifies a separate Helmholtz equation at each $x, y$ coordinate for the "local normal modes." 
Using these principles, we can create a crude model for a NIW train using a series of "sudden interface" perturbations that represent the displaced thermocline at each soliton location. Figure 2.1 illustrates three conditions where refraction, defocusing, and focusing effects dominate. Gray bars represent soliton perturbations in horizontal wavenumber $\left(k_{r m}\right)$ space, for a single mode $m$, at a specific frequency $f$. Here the solitons decrease the wavenumber values by a small amount, with $k_{r m 0} \approx 1.25$ and $\Delta k_{r m} \approx-0.01$ (these values chosen from real SW06 data for Mode 3 at $300 \mathrm{~Hz}$ ). The perturbation profile varies with cross-range ( $y$-axis) and remains valid across range $(x$ axis). Modal rays spread from the source at $x_{s}=y_{s}=0$ and at various launch angles for the corresponding mode $m$ and frequency $f$. The top-most panel represents a refraction scenario, which would occur prior to the NIW reaching the direct acoustic path. In certain conditions the launch angles strike the NIW crest at low grazing angles, below the critical angle, and refraction occurs. This is determined by Snell's law, written in terms of the modal eigenvalues,

$$
k_{r m} \cos \theta=k_{r m}^{\prime} \cos \theta^{\prime},
$$

where the prime indicates a transition to a second (perturbed) state. The critical angle for complete refraction is therefore

$$
\theta_{c}=\cos ^{-1}\left(\frac{k_{r m}^{\prime}}{k_{r m}}\right) .
$$

For most cases, the mode-dependent critical angle due to a NIW is on the order of $4-5^{\circ}$. The center panel shows a defocusing condition, which would occur as the NIW reaches 
the source. Here the rays are vectored outwards, away from the direct acoustic path, resulting in energy being dispersed laterally. Lastly, the bottom panel shows a focusing condition, where two solitons create a horizontal sound channel, trapping acoustic energy between them.

For each scenario, locations of possible acoustic receivers are positioned near-parallel to the NIW crests. Even with mild variations in receiver position; there can be substantial differences in received acoustic energy. For example, in the case of the refraction condition, a receiver positioned at $+3^{\circ}$ off-axis would likely receive increased acoustic energy due to additive refracted energy, whereas a receiver positioned at $-3^{\circ}$ off-axis would be shielded from this energy. Receiver position plays an even stronger role in the focusing scenario. Here only the receiver positioned directly parallel to the NIW front $\left(0^{\circ}\right)$ receives energy trapped between solitons. We can hypothesize that even very small angle differences can create substantial impacts upon the magnitude of received energy fluctuations. Moreover, as the NIW traverses the sound-field over space and time, an even more complicated scenario can be considered.

A more lifelike NIW representation is shown in Figure 2.2, derived from a mathematical "dnoidal" solution, which allows for a series of bore-like depressions in the thermocline. ${ }^{31,21}$ Using this NIW approximation, we define a general scenario for the parallel case. We assume the bottom and surface conditions remain flat and homogeneous, so that variability in the water-column creates the largest impact upon the acoustic field. Additionally, we assume that the internal wave train is fully developed and that it does not undulate quickly - or is acoustically "frozen." We further assume that the leading edge has no curvature along the crest and remains range independent 
along the acoustic track. Although, it should be noted that curvature in the wave-crests or sharply terminated internal waves do exist, and this scenario is acoustically important. ${ }^{27}$, 28,29

Figure 2.2 also demonstrates horizontally refracted modal rays for an acoustic source at different locations relative to the NIW. This can be interpreted as an acoustic source being towed through the NIW, or alternatively (and similar to the data presented in this paper), a NIW propagating past a moored acoustic source. The rays depicted in Figure 2.2 represent an arbitrary mode number $m$, at an arbitrary frequency $f$. The acoustic path for each modal ray is determined by an index of refraction $n_{m}$, as indicated by equation (2.5). Therefore, sound speed differences in the horizontal plane due to NIW activity can greatly influence the path traveled by each modal ray. Since the total pressure field will be the summation of all trapped modes in the water column, and each mode travels an individual path, a multipath constructive and destructive interference pattern will occur. Further, in the case of a broadband acoustic source, where the modal rays are also frequency dependent, an even more complicated interference pattern is likely to transpire. In general, modal refraction increases with higher mode numbers. ${ }^{23}$

While this constructive and destructive interference pattern will cause an element of stochastic uncertainty in the acoustic data, a deterministic aspect can be applied by expanding the simplistic scenarios offered in Figure 2.2. As the NIW passes through the sound field, time-varying acoustic fluctuations will become apparent, and regimes can be defined based upon the position of the NIW relative to the acoustic track. These regimes, shown in Figure 2.2, separate the dominant physical processes versus time, and are labeled the quiescent regime, the refraction regime, and the NIW interference regime - 
the latter being a combination of focusing and defocusing effects. Note that Figure 2.2 delineates these regimes at different depths in the water column for clarity only - the modal rays are not depth dependent. At first (zero time or zero range), the approaching internal wave will have little to no impact upon the acoustic field, simply because it is too far away. This can be termed the quiescent ${ }^{32}$ regime, where any fluctuations are due to stochastic scattering or a mildly fluctuating water column. As the NIW's leading edge approaches the source, modal rays will refract off the first soliton. This refraction regime will create an interference pattern in the horizontal plane akin to a Lloyd's Mirror phenomenon. ${ }^{33,30,34}$ At the moment the leading soliton centers itself over the acoustic source, defocusing spreads the sound outwards in an "anti-duct" fashion. Then, as the wave progresses and solitons trap the acoustic source, focusing occurs in a horizontal "duct." Combined, both defocusing and focusing effects due to several solitons in the NIW train create a complicated and strong interference regime. As the NIW fully propagates and only vestiges of the soliton train are left, fluctuations will taper off. It is hypothesized that in some instances, depending on the strength and structure of the NIW, "back-end" refraction could occur from larger solitons at the tail-end of the NIW train.

The general case defined here is a simplistic one and overlooks complications in the ocean environment, such as non-uniform bathymetry, inhomogeneous bottom properties, and surface scattering. Further, non-uniformity in the internal wave field, including range dependence along the NIW (i.e. curvature, waning or truncated solitons), and crossing NIWs create even more possibilities for fluctuations in the acoustic field. Nonetheless, the time-varying regimes suggested by this general case have become 
apparent during analysis of experimental data from the SW06 experiment, some of which are offered in this paper.

\subsection{SW06 EXPERIMENTAL SETUP}

The SW06 experiment took place during the summer of 2006 off the New Jersey coast, with a primary goal of advancing our understanding of acoustic propagation in shallow water. The multi-institutional endeavor was sponsored by the Office of Naval Research, and included numerous research vessels, scientific moorings, and principal investigators. The experimental location was proximate to the continental shelf break, near the site of previous acoustic studies ${ }^{10}$, and was ideally situated in an environment where internal waves are known to occur. An excellent overview is provided by Newhall, et al. ${ }^{13}$ for detailed information regarding the SW06 experiment setup as a whole.

As part of the SW06 experiment, the University of Delaware's Research Vessel (R/V) Sharp ${ }^{35}$ transmitted various acoustic signals to create persistent measurements during the passage of NIW trains. During a three week deployment, the R/V Sharp positioned itself at varying ranges and bearings to the Woods Hole Oceanographic Institute's "Shark" receiving vertical line array (VLA) while transmitting low-frequency acoustic signals using a J-15 source. Throughout the deployment, the R/V Sharp detected the surface expression of 58 internal wave events on marine RADAR. Figure 2.3 shows the SW06 experimental area, including R/V Sharp locations C1B and C1D. These two locations were strategically chosen based upon the heading of previously experienced NIWs - such 
that any newly experienced NIW events would likely be nearly parallel to the acoustic track created by the R/V Sharp J-15 source and the WHOI receiving array.

The two NIW events analyzed in this paper were labeled "Event 44" and "Event 47" as they were the $44^{\text {th }}$ and $47^{\text {th }}$ events experienced during the R/V Sharp's deployment, and occurred at locations $\mathrm{C} 1 \mathrm{~B}$ and C1D respectively. During both NIW events, the J-15 terminated transmissions while other SW06 acoustic sources were activated. Although cessation of the $\mathrm{J}-15$ source unfortunately caused some sparseness in the acoustic data, the R/V Sharp's persistent transmissions successfully captured acoustic modulations due to NIW activity. The experimental configurations for Event 44 and Event 47 were virtually identical, aside from different locations for the acoustic source. It was primarily the dissimilarity in the oceanographic environment that created uniqueness between the two NIW events (and resulting datasets). During both experimental configurations, the WHOI receiving VLA was collocated with environmental mooring SW54 $\left(39^{\circ} 1.252^{\prime} \mathrm{N}\right.$, $\left.73^{\circ} 2.983^{\prime} \mathrm{W}\right)$. The VLA consisted of 16 elements spanning the lower portion of the water column between 13 and 80 meters. In the case of Event 44, the R/V Sharp deployed a J-15 acoustic source 74 meters below the surface, where the water was 78-80 meters deep, at location C1B (39 $\left.9^{\circ} 6.8^{\prime \prime} \mathrm{N}, 72^{\circ} 57^{\prime \prime} 26.6^{\prime \prime} \mathrm{W}\right)$. This location was $15 \mathrm{~km}$ away from the WHOI receiving VLA and at a bearing of $212^{\circ}$. The bathymetry between the source and receiver remained relatively flat, with only small depth differences along the acoustic track (varying between 77 and 82 meters). A sequence of 23 broadband (50$450 \mathrm{~Hz}$ ) linear frequency modulated chirp signals, each 0.7 seconds long, were persistently transmitted every three minutes, except during times other SW06 acoustic sources were activated. In the case of Event 47, the J-15 acoustic source was deployed 
50 meters below the surface, where the water was $82-84$ meters deep, at location C1D $\left(39^{\circ} 7^{\prime} 27.4^{\prime \prime} \mathrm{N}, 72^{\circ} 56^{\prime} 16.6^{\prime \prime} \mathrm{W}\right)$. This location was also $15 \mathrm{~km}$ away from the WHOI receiving array, but at slightly different bearing of $220^{\circ}$. An identical chirp signal as Event 44 was transmitted, although consecutively, rather than in separate sequences.

\subsection{OCEANOGRAPHIC BACKGROUND AND MEASUREMENTS}

Before examining environmental data specific to Events 44 and 47, some larger scale oceanography should be considered, which provides a setting for localized NIW phenomena. Throughout the SW06 experiment, the evolution of the continental shelf's hydrography and stratification played a dominant role - influencing the NIW and thermohaline intrusion activity experienced during certain timeframes. Meandering of the off-shelf front, and the occasional existence of small warm core rings (sometimes referred to as off-shelf eddies), caused interesting three-dimensional advection effects in the experimental area.

During the month of August 2006, two eddy masses (warm core rings) existed off the continental shelf - the first roughly between August 6-10, and the second starting around 25 August. Events 44 and 47 occurred between these eddy masses, on August $13^{\text {th }}$ and $16-17^{\text {th }}$ respectively. This timing is important for two reasons - firstly, water intrusions are associated with these eddies, and secondly, they affect the stratification of the thermocline. During timeframes off-shelf eddies exist, both surface and pycnocline intrusions can occur, in essence mixing the thermocline. However, for a period between these eddies (August 12-20), stronger stratification of the thermocline existed, where a less-saline surface layer was present due to local freshwater river discharge. Additionally, wind-forced Ekman transport during this period helped strengthen the 
thermocline, where Northeast (positive) wind stresses pushed the surface-water offshore, and in turn pulled the more saline off-shelf water along the bottom of the continental slope, creating a thermohaline bottom intrusion - often called "the foot of the front." In general, this process delivers the most salinity onto the shelf ( $34 \mathrm{ppt})$ and creates even stronger stratification in the thermocline ${ }^{36}-$ a crucial factor relative to the existence and strength of NIWs.

NIWs occur throughout the world and are primarily caused by internal tidal forcing and interactions with a non-uniform ocean bathymetry. They are well known to occur in the SW06 experimental area, and because the New Jersey shelf is broad with a mild slope, NIW evolution occurs gradually. They are physically important contributors to mass transport and heat flux distribution into the water column - in fact, NIW measurements throughout August accounted for roughly 50\% of heat flux across the pycnocline. A notable six day time span between August 17- 22 contained a manifestation of larger NIWs, which was slightly unexpected because this was concurrent with the weaker neap barotropic tide. ${ }^{37}$

Given these mesoscale oceanographic conditions, we see that Event 44 took place during a time when the pycnocline was strongly stratified, and a salty thermohaline bottom intrusion existed. Event 47 occurred at the onset of a mid-water column intrusion moving along the shelf, towards the SW06 experimental area. Further, Event 44 happened just prior to, and Event 47 at the cusp of, the some of the greatest internal wave activity measured during the SW06 experiment. These important oceanographic factors set the stage for these discrete NIW events, and help interpret environmental sensor data relative to our acoustic analysis. 
In order to measure the NIW activity for Events 44 and 47, we rely upon both moored and ship-borne sensors. During the experiment, marine RADAR served as one of the most useful tools to infer NIW presence, because during calm seas, the NIW surface expression can become apparent on RADAR display. From this imagery, one can estimate the general properties of the NIW, such as: whether it has curvature along the crest, the approximate number of solitons within the NIW train, the relative bearing and speed of propagation, and when it reaches the deployed acoustic source. Figure 2.4 shows three examples of RADAR imagery overlaid upon the SW06 experimental area these snapshots include Event 44 before it arrived, as it passed beneath, and passed beyond the research vessel and acoustic source (between 3:00 and 11:00 GMT on August $14^{\text {th }}$ ). We notice that the NIW crest was approximately parallel to the acoustic track, and as the NIW reached the acoustic source, the wave-fronts show little-to-no curvature. Interpreting several images over time, we can infer that Event 44 propagated relatively slowly $(0.5 \mathrm{~m} / \mathrm{s})$, northwest by west at a bearing of $308^{\circ}$.

In addition to marine RADAR, the R/V Sharp was equipped with a $300 \mathrm{kHz}$ RD Instruments downward looking Acoustic Doppler Current Profiler (ADCP), which probed the water column, and allows us to estimate the overall strength and structure of the NIW as it passed beneath the ship - determining the NIW's time-dependent profile relative to the acoustic source. The top panel of Figure 2.5 displays the ADCP's Echo Sounder channel recorded during Event 44. Here, a strongly oscillating pycnocline is apparent between 05:20 and 07:30 GMT, where at least 14 well-defined soliton depressions, roughly 15 meters in amplitude, indicate Event 44 was a strong Mode-1 structured NIW. These stronger depressions are then followed by several smaller and lesser-resolved 
pycnocline oscillations. In comparison, the top panel of Figure 2.6 shows that Event 47 was again a Mode-1 structured wave, but consisted of only three solitons, each decreasing in amplitude, from roughly 17,14 , to 11 meters. Recently, similar data using upward-looking Echo Sounders has been further interpreted to estimate the overall structure of passing NIWs. ${ }^{38}$

The center and bottom panels of Figure 2.5 and Figure 2.6 provide the ADCP velocity channels, illustrating the overall particle velocity magnitude and the extracted vertical component. For both Events 44 and 47, the maximum particle velocity is roughly between $0.5-0.6 \mathrm{~m} / \mathrm{s}$, and regions of high velocity appear in concert with the soliton depressions. Similarly, the vertical velocity aligns well with solitons, where the upward (and downward) particle velocity reaches up to $0.2 \mathrm{~m} / \mathrm{s}$ prior to (and following) each depression. Velocity data from Event 47 indicate a more complex scenario than Event 44, suggesting there may have been other oceanographic mechanisms creating additional disturbances to the velocity profile - possibly the onset of a mid-water column intrusion. In both cases, and in general, these upward and downward currents help create the surface "rip" that helps make NIWs detectable by marine RADAR - and also create important mixing and transport aspects that are of extreme interest to the physical and biological oceanographic communities.

During each NIW event, the R/V Sharp also performed Conductivity-TemperatureDepth (CTD) casts, employing a repeated up-and-down "yo-yo" fashion, in order to ascertain sound speed variability in the water column. Figure 2.7 and Figure 2.8 show measured temperature and salinity profiles as well as the computed ${ }^{39}$ sound speed for each event. During Event 44, sound speeds fluctuated between 1487-1530 m/s at 10-35 
meters depth, while Event 47 sound speeds varied between 1494-1530 m/s at 15-45

meters depth. An outlying CTD cast in Figure 2.8 stems from Event 47's leading soliton arriving at the $\mathrm{R} / \mathrm{V}$, and represents a brief period of increased temperature, decreased salinity, and faster sound speed, signifying upper-layer waters being pushed deeper into the water column. To display the Event 44 CTD casts versus time, and the passage of the NIW beneath the ship, these same sound speed data are plotted as a color scalogram in the far right panel of Figure 2.7. These casts show relatively uniform profiles until 05:40 GMT, when an onset of strong oscillations occur, where the sound speed minimum is quickly pushed from roughly 25 to 40 meters. The timing and structure of this variability corroborates extremely well with both the marine RADAR and ADCP measurements. Additionally, the "foot of the front" is evidenced by a region of increased sound speed along the bottom of the water column.

Accompanying these ship-borne sensors, NIW activity was recorded by a series of environmental moorings placed throughout the experimental area - each possessing a thermistor array that adequately detected thermocline perturbations in the water column. The two environmental moorings relevant to Events 44 and 47 are SW32, located midway between the source and receiver, and SW54, collocated at the receiving VLA.

The lower three panels of Figure 2.9 compare Event 44's internal wave structure at three locations along the acoustic track. The ADCP echo (previously shown in Figure 2.5 ), indicates NIW activity at the acoustic source. A representative profile at a midway point between the source and receiver is offered by interpolated sound speed measurements recorded by environmental mooring SW32 - located $5.43 \mathrm{~km}$ from the acoustic source at a bearing of $221^{\circ}$, or 844 meters-off axis from the acoustic track. 
SW32 captured Event 44 as a well-developed NIW train, consisting of 12 pronounced solitons, followed by a series of smaller and less regular thermocline depressions. Both the ADCP Echo and SW32 show a strikingly similar representation of Event 44, indicating strong uniformity in the NIW's structure over this section of the acoustic track. Although SW32 was slightly off-axis, this discrepancy accounts for only a seven minute time-shift when associating soliton arrivals between these two locations. In comparison, the period between solitons was roughly twelve minutes. At the receiving VLA, SW54 captured slightly weaker NIW activity starting at approximately 4:30 GMT, indicating Event 44 reached this location prior to the acoustic source. Here, only six strong soliton depressions are evident, suggesting the NIW was less evolved at this period in time, that some of the trailing solitons tapered with range, or a combination of both factors.

Relating internal wave activity at the source, receiver, and midway point we can deduce some important characteristics regarding Event 44 relative to the acoustic track. Firstly, environmental data indicates the NIW arrived at the receiver about one hour prior to the acoustic source, and that the leading NIW wave crest can be well-approximated by a straight line. We can estimate a forward propagation speed of $0.5 \mathrm{~m} / \mathrm{s}$ and a bearing difference between the leading edge of the NIW and the source-receiver path, $\alpha$, at approximately $5-6^{\circ}$.

In contrast to Event 44 , Event 47 was a more localized and shorter duration phenomenon. Measurements at the receiving VLA on SW54 (not shown) indicate irregular NIW activity between 21:45 and midnight GMT on August $16^{\text {th }}$. Environmental mooring data captured by SW32 indicate a very strong single soliton arrival at 23:20 GMT, following by a lowered thermocline lasting through the remainder of the day 
(Figure 2.10 ). As previously mentioned, NIW activity at the source showed three discrete solitons passing beneath the ship, and marine RADAR imagery indicated two straight line fronts with the first wave crest measuring between $205-210^{\circ}$ as it reached the vessel, creating a bearing difference between the acoustic track and the propagating NIW in the realm of $\alpha=10-15^{\circ}$. In this scenario, using a straight-line approximation, the NIW reaches the acoustic source before the receiving VLA. Given this relatively larger angle, the acoustic data could be interpreted as falling within the adiabatic regime, and horizontal effects would not appear to dominate. However, because the acoustic data appear to display some important defocusing properties relevant to the horizontal regime, it is also included in this investigation.

\subsection{ACOUSTIC MEASUREMENTS AND ANALYISIS}

\section{A. Broadband energy metrics}

In order to quantify the intensity fluctuations that occurred during NIW activity, we employ methods described by Duda et al. ${ }^{40}$ and Fredericks et al. ${ }^{41}$ which have previously been used to treat acoustic data from the PRIMER and ASIAEX experiments. In these analyses, acoustic data spanning several days were quantified by decomposing intensity and energy values into tidal and subtidal frequencies, thereby appraising the impact of ocean processes in an all-inclusive convention. Here, we use the same metrics to analyze discrete NIW events and correlate them with local oceanographic processes to better understand the variability of the acoustic field during much shorter timescales (hours versus days). 
Prior to performing metrics upon the chirp signals, receptions were high-pass filtered $\left(f_{c}=100 \mathrm{~Hz}\right)$ to limit possible low-frequency shipping and environmental noise contributions. Each chirp arrival (or transmission number) $k$, at each hydrophone depth $z$, was pulse compressed using the match filtered output $\psi$ of the complex demodulates, i.e. $I(\tau, z, k)=|\psi(\tau, z, k)|^{2}$. In this case, the pulse compressed signal $\psi$ relies upon the analytic signal derived from quadrature amplitude demodulation, which is then correlated with the original transmitted signal. This process greatly increases SNR, however as a final quality check, all signals were visually inspected such that chirp arrivals overshadowed by noise were manually removed.

These data then served as the basis for statistical metrics - temporally integrated energy $I_{\tau}$, being the primary tool to correlate acoustic data to oceanographic evidence of NIW activity. This metric provides insight into the depth dependence of acoustic energy, and also helps to discriminate time varying energy changes over the water column, such as mode coupling. To calculate temporally integrated energy, each pulse-compressed arrival is integrated over the energetic region of the signal $\tau$, with these time limits carefully chosen in order to reduce residual noise contributions, but to also incorporate multipath arrivals.

$$
I_{\tau}(z, k)=\int I(\tau, z, k) d \tau
$$

Each dataset created by Events 44 and 47 were then normalized such that the overall mean is one; $\left\langle I_{\tau}\right\rangle=1$. Normalizing these data removes gain created by the pulse compression process, and also serves to highlight signal intensifications $(I>1)$. 
However, caution should be used when interpreting signal fades $(0<I<1)$, as they may be more difficult to discern. The top panel of Figure 2.9 shows temporally integrated energy receptions during Event 44 on a linear scale for all hydrophones of the WHOI VLA. For direct comparison, these data are plotted against the same timescale as oceanographic information. The top panel of Figure 2.10 shows temporarily integrated energy for Event 47 in comparison to NIW activity at environmental mooring SW32. Again, breaks in these data are due to temporary pauses in the R/V's transmissions during other SW06 source activity. The normalized temporally integrated energy for both Events 44 and 47 are well-fitted by a lognormal distribution, which agrees with previous observations given by Fredericks et al. ${ }^{41}$ for acoustic data influenced by NIW activities (high-frequency oceanographic processes).

From this point forward we primarily focus upon Event 44, mainly because the environmental measurements confirm NIW activity along the entire acoustic track. Therefore, this dataset is well-suited to investigate energy fluctuations due to the NIW's presence. Figure 2.11 shows $I_{\tau}$ plotted in four separate manners to demonstrate different aspects of these data. The top-left panel shows $I_{\tau}$ for the entire Event 44 dataset versus transmission index, which is a proxy for time. The top right panel shows a normalized histogram of these data, which ensemble, consists of 16100 samples. Overlaid is a fitted lognormal probability density function (PDF) yielding a lognormal mean $\hat{\mu}$, of 0.986 , and a lognormal variance $\hat{\sigma}^{2}$, of 0.417 . In order to help interpret the energy distribution versus depth, $I_{\tau}$ receptions upon each hydrophone are shown through a color scalogram in the bottom left panel. Although the VLA contained 16 hydrophones, only the top 14 
are considered since hydrophones 14-16 were approximately collocated, near the seafloor. The bottom right panel displays a statistical boxplot for each phone, where the circular mark represents the median, the edges of the box the $25^{\text {th }}$ and $75^{\text {th }}$ percentiles, and the whiskers extending to the furthest points not considered outliers. The outliers are plotted as individual points. Therefore, it is important to note that the highest energy points that are visually striking throughout these data are in fact outliers, and highlight fluctuations that are well above the mean. For this reason, interpreting PDFs which represent sections of these data may be a more appropriate way to infer the general impact of physical processes on the acoustic field. (We will offer an interpretation of these data based upon this approach in the following section.) The boxplot also infers that at least two acoustic ducts appear in the water column. Both the mean values, as well as the outliers, indicate the energy in the water column peaked at roughly 20 and 60 meters. Given this interesting depth dependence, modal decomposition is a logical next step.

\section{B. Modal decomposition}

Broadband modal beamforming was carried out according to the methods described by Crocker et al. ${ }^{42}$ such that the signals were match-filtered and weighted by their modal components in the frequency domain. A non-match-filtered LFM chirp signal received upon the eighth hydrophone of the WHOI VLA (39 meters deep) is displayed at the far left panel of Figure 2.12 as a representative example of all Event 44 data. This acoustic signal was recorded at the start of the ramping feature during Event 44; it was band-pass filtered between 70 and $325 \mathrm{~Hz}$ in order to remove low frequency noise, and also to remove a $330 \mathrm{~Hz}$ tone (instrument noise) that was persistent throughout these data. The 
spectrogram shows a splitting at the "foot" of the chirp (below $150 \mathrm{~Hz}$ ) which indicates separate modal arrivals, and modal dispersion in the acoustic waveguide. Additionally, some patchiness of the chirp arrival above $250 \mathrm{~Hz}$ appears, suggesting additional variability in the higher frequency portion.

Broadband modal decomposition of this signal is displayed in the right-most panels of Figure 2.12 for the first five modes, serving to explain some of the frequency dependent variability. For consistency across plots, the color scale for each modal arrival is normalized and identical. Specific to this particular sample, Mode 1 carries energy in the lowest part of the frequency spectrum (less than $200 \mathrm{~Hz}$ ), whereas Mode 5 carries energy in the upper part of the spectrum (greater than $200 \mathrm{~Hz}$ ). Modes 2, 3, and 4, appear to carry energy throughout the entire 70-325 Hz band. The modal decomposition shows splitting at the foot of the chirp can be attributed to separate mode arrivals. While Mode 1 shows a steady LFM chirp arrival (similar to the transmitted signal), Modes 2-6 show increasing delays at frequencies below $200 \mathrm{~Hz}$. The broadband mode arrivals for this signal are also shown in Figure 2.13, and visual interpretation infers Modes 2 and 3 appear to carry more energy than others.

The top panel of Figure 2.14 shows the modal energy distributions for the duration of Event 44, using the frequency bands identified in the sample chirp signal. The lognormal mean and variance for each modal distribution is also annotated. Similar to the approach employed in calculating $I_{\tau}$, these signals have been match-filtered, and the energy for each modal component was calculated by integrating under the energetic region of the received mode arrival. For the entire 70-325 Hz spectrum, Modes 1-3 dominate energy delivery and signal variability upon the VLA. This is also true for the lower frequency 
band $(70-200 \mathrm{~Hz})$, shown in the lower-left panel of Figure 2.14 . However, the lowerright panel of Figure 2.14 indicates that Modes 3-5 dominate energy delivered to the VLA for frequencies above $200 \mathrm{~Hz}$. The frequency dependence of this broadband signal explains why Mode 3 tends to carry the most energy during the totality of Event 44, as it carries energy in both the lower and upper bands of the chirp signal.

\section{Qualitative modeling}

In order to better understand the fluctuations we see during NIW activity, we turn to three-dimensional propagation modeling. The advantage of a model is that any uncertainty from oceanographic processes that exist in our measured data can be removed. However, modeling the time dependence of a NIW traversing the sound field is computationally expensive, requiring many individual model runs. Additionally, modeling the broadband nature of our signals also increases the computational burden. Therefore, we consider three instances for an idealized scenario that is directly comparable to Event 44, and also directly comparable to the refraction, defocusing, and focusing conditions described earlier.

The three dimensional KRAKEN normal mode $\operatorname{program}^{43}$ was employed to simulate the behavior of individual modes during Event 44. To reproduce the NIW, a simple constant-depth water column of 80 meters was chosen, with bottom parameters representative of the SW06 environment. ${ }^{44}$ A $300 \mathrm{~Hz}$ source, located at 74 meters depth simulated the J-15 deployed from the R/V Sharp - this frequency chosen because it is roughly the center of the real-world chirp signal's bandwidth. The primary environmental input for this model was an interpolated sound speed profile, derived from measured data recorded by environmental mooring SW32 during Event 44. Because 
SW32 was situated midway between the source and receiver, we assume it represents a general profile of Event 44 across the entire acoustic track. We consider a straight line NIW front, letting the sound speed profile vary along cross-range ( $y$-axis) with no variation in the range dimension ( $x$-axis).

The left panel of Figure 2.15 shows the interpolated sound speed profile supplied to the KRAKEN program along the depth and cross-range dimensions. The three adjacent panels represent the corresponding sound field across range and cross-range. For each instance, the acoustic source (marked by a star) is positioned at a different location relative to the NIW sound speed profile, and horizontally refracted modal rays for the first five modes are depicted at various launch angles from the source location. In the first example, the source is positioned 200 meters before the leading edge of the NIW, representing a refraction condition. Higher modes have higher critical angles, illustrated by the fact that the higher order modes are more easily refracted, and the lower order modes penetrate the leading soliton more easily. In the second example the source is situated at the center of the first soliton, creating a defocusing condition. As in the refraction scenario, the lower order modes do not refract as easily. Therefore, an acoustic track directly parallel to the NIW front would receive less energy from higher order modes when compared to an unperturbed environment. However, an acoustic track that is slightly off-axis (such as the condition described in Event 44) may actually receive more energy in this scenario because the defocusing event has diverted some energy obliquely. The third example portrays a focusing condition, where the source is positioned between two solitons, creating a horizontal waveguide. Here rays for all five modes are refracted in an oscillatory pattern along the parallel track. In this condition, an 
acoustic track exactly parallel to the NIW would expect a significant level of energy delivery. However, if the source-receiver path is slightly askew from parallel (outside of the horizontal waveguide), a decrease in energy would be anticipated since a significant portion has been vectored between the solitons.

\subsection{INTERPRETATION OF OBSERVATIONAL DATA}

The SW06 experiment happened to be one of the largest oceanographic field experiments in history, with an unprecedented number of oceanographic sensors - not only including moorings and ship-borne assets, but also Autonomous Underwater Vehicles (AUVs), air-based, and space-based assets. These resources provided a unique opportunity to interpret acoustic data in concert with oceanographic data. However, even with this rich environmental dataset, our examination of NIW activity during Events 44 and 47 is sufficiently under-sampled to draw any firm conclusions when correlating oceanographic and acoustic measurements. Therefore, we offer an interpretation of the observational data, and hypotheses for fluctuations that we see. To further examine Events 44 and 47 we align them to local oceanographic processes, and statistically decompose select data into regimes where dominant acoustic processes are likely. We specifically search for areas where NIW modulations of the sound field are apparent and fall within the quiescent, refraction, or NIW interference, regimes previously suggested. We also point to possible instances of focusing and defocusing.

\section{A. Comments upon Event 44}

Taking a cursory glance at acoustic data received upon the VLA during Event 44

(Figure 2.9 ), one notices a significant ramping of acoustic energy between 3:00 and 5:40 GMT, leading up to the NIW arriving at the acoustic source. Closer inspection reveals 
some intricacies associated with this escalating energy. Prior to any NIW activity at the receiver, energy appears to steadily intensify throughout the water column. Examining these receptions versus transmission index $(k=0-350)$ in the top left panel of Figure 2.11 the signals exhibit a seesaw pattern where the energy spikes and fades versus time. This feature can likely be explained by a Lloyd's Mirror interference pattern in the horizontal plane. Then, for a brief period of time, while NIW activity was evident at the receiver but had not yet arrived at the acoustic source, the overall energy received upon the VLA is somewhat suppressed. This suppression occurs when the NIW started to cross into the acoustic track, likely diverting energy away from the receiving array. Prior to the NIW reaching either the acoustic source or receiver (4:30 GMT), we propose that horizontal refraction processes dominate, and consequently categorize these data into a refraction regime. It should be noted that refraction over 1.5 hour duration is not typical, as this phenomenon is expected to be on the order of 30 minutes. An increase in ambient noise coincides with the NIW's arrival, which is an important factor to consider. After a separate noise analysis, we remain confident these data accurately reflect the increase in signal energy, and that we have sufficiently mitigated any possible noise corruption. APPENDIX A expands these arguments in more detail.

At 5:40 GMT a collection of high energy signals is coincident with the NIW arriving at the acoustic source (indicated by a white dotted line superimposed upon the ADCP echo plot of Figure 2.9 ) which we propose to be a focusing phenomenon. Because the NIW was not completely parallel to the acoustic track we cannot definitively assume that this is a strict focusing event, and a few reasonable scenarios can be suggested where energy is directed towards the VLA. One possibility is that a ducting of energy did occur 
between two solitons, and energy travelled along this acoustic waveguide towards the general direction of the VLA. If the waveguide became less defined (which is evident from NIW activity captured at the VLA), the energy would begin to spread down-range from the acoustic source, casting energy in the receiver's general direction. This scenario would be similar to that of a truncated NIW suggested by Lin et $a l .{ }^{28}$ Another possibility is that strong refraction or defocusing at the acoustic source directed a large amount of energy towards the VLA early on in the acoustic path. Although some speculation is at work here, we hypothesize focusing plays a dominant role, and categorize this portion of Event 44 into a focusing regime.

Following this, a period of subdued energy was captured between 6:00 and 8:00 GMT. Interestingly, this two hour period occurs during the period of strongest NIW activity, indicating these strongest solitons vectored some energy away from the VLA. After 8:00 GMT the energy then evolves into a vacillating sound field with increased seesawing energy spikes. These fluctuations could again be due to some reasonable scenarios. One conjecture is that the strongest solitons no longer existed in the acoustic track, and while the smaller soliton depressions caused substantial fluctuations in the sound field, they could no longer vector comparable energy away from the VLA. A second supposition is that back-end refraction off the larger solitons that had recently passed the acoustic track may exist, thereby creating stronger fluctuations when compared to peak NIW activity. Because this entire period of time (after 6:00 GMT) occurs while NIW activity crosses the acoustic track, and the acoustic data appears very erratic, we categorize this portion of data as a NIW interference regime. 
Revisiting Figure 2.11, we recall that the entire Event 44 acoustic dataset consisted of 16100 samples, well-fit by a lognormal PDF with lognormal mean $\hat{\mu}=0.986$, and lognormal variance $\hat{\sigma}^{2}=0.417$. This serves as a comparison point for breaking the data into the proposed refraction, focusing, and NIW interference regimes. Figure 2.16 shows the lognormal PDFs for these data separated into each regime to show the general differences or similarities between these areas of acoustic data. Based on the fitted distributions it becomes apparent that focusing effects dominate both signal intensification and signal variability. Another noteworthy point is that refraction effects prior to the NIW's arrival cause similar variability as NIW interference effects. The important argument to be made here is that when considering acoustic fluctuations in the presence of internal waves, signal intensification and variability prior to the NIW's arrival can be considered equivalently significant as the NIW passing over the acoustic track. This scenario is especially likely if the acoustic track is not exactly parallel to the NIW, and focusing between solitons may actually divert energy away from the receiver.

The bottom left panel of Figure 2.11 illustrates energy levels upon each hydrophone, showing depth dependence. At the start of the ramping feature, an acoustic duct steadily increases on the fifth hydrophone (at 28 meters) and then abruptly ceases as the NIW crosses into the acoustic track (prior to $k=300$ ). This may indicate that the escalating energy could be an intensifying mode as the NIW draws closer to the acoustic track. As the NIW enters the acoustic track ( $300>k>450$ ), energy dominates at various depths, indicating that only certain modes are traveling through the leading edge of the NIW, or perhaps mode coupling is causing a vertical redistribution of energy. As the proposed focusing event occurs ( $450>k>500$ ), there is an increase in energy throughout the 
water column, and significant intensification on hydrophones 2, 4, 11, and 12 (at 16, 24, 54 and 61 meters). After transmission 500, and during the NIW interference regime, energy is spread throughout the water column, with some evidence of escalating energy at the end of NIW train, again upon hydrophones 2, 4, 11 and 12.

The mean and variance values for mode-dependent energy partitioned into the refraction and NIW interference regimes are listed in Table 2.1, and for upper and lower frequency bands in Table 2.2 and Table 2.3. The focus regime is omitted because the number of receptions during this period of time does not provide satisfactory sampling for both modal and statistical decomposition. In the refraction regime, higher frequencies and higher modes appear to deliver more energy to the VLA. The NIW interference regime lends itself to less conclusive modal and frequency contributions, but follows the same trend as the overall dataset, where higher modes deliver more energy at higher frequencies, and lower modes deliver more energy at lower frequencies. 
Table 2.1 Event 44 broadband modal decomposition (70-325 Hz)

\begin{tabular}{|c|c|c|c|c|c|c|}
\hline \multirow{2}{*}{ Mode } & \multicolumn{2}{|c|}{ All Regimes } & \multicolumn{2}{c|}{$\begin{array}{c}\text { Refraction } \\
\text { Regime }\end{array}$} & \multicolumn{2}{c|}{$\begin{array}{c}\text { NIW } \\
\text { Interference }\end{array}$} \\
\cline { 2 - 7 } & $\hat{\mu}$ & $\hat{\sigma}^{2}$ & $\hat{\mu}$ & $\hat{\sigma}^{2}$ & $\hat{\mu}$ & $\hat{\sigma}^{2}$ \\
\hline 1 & 1.13 & 0.91 & 0.45 & 0.04 & 1.39 & 0.80 \\
\hline 2 & 1.26 & 0.88 & 0.56 & 0.04 & 1.53 & 0.88 \\
\hline 3 & 1.34 & 0.77 & 0.75 & 0.08 & 1.53 & 0.75 \\
\hline 4 & 0.80 & 0.14 & 0.62 & 0.08 & 0.85 & 0.11 \\
\hline 5 & 0.44 & 0.04 & 0.44 & 0.06 & 0.42 & 0.02 \\
\hline
\end{tabular}

Table 2.2 Event 44 broadband modal decomposition - lower band (70-200 Hz)

\begin{tabular}{|c|c|c|c|c|c|c|}
\hline Mode & \multicolumn{2}{|c|}{ All Regimes } & \multicolumn{2}{c|}{$\begin{array}{c}\text { Refraction } \\
\text { Regime }\end{array}$} & \multicolumn{2}{c|}{$\begin{array}{c}\text { NIW } \\
\text { Interference }\end{array}$} \\
\cline { 2 - 7 } & $\hat{\mu}$ & $\hat{\sigma}^{2}$ & $\hat{\mu}$ & $\hat{\sigma}^{2}$ & $\hat{\mu}$ & $\hat{\sigma}^{2}$ \\
\hline 1 & 1.21 & 1.25 & 0.42 & 0.03 & 1.53 & 1.11 \\
\hline 2 & 1.35 & 1.35 & 0.51 & 0.04 & 1.67 & 1.29 \\
\hline 3 & 1.38 & 1.13 & 0.68 & 0.10 & 1.61 & 1.08 \\
\hline 4 & 0.71 & 0.18 & 0.51 & 0.10 & 0.77 & 0.13 \\
\hline 5 & 0.32 & 0.03 & 0.32 & 0.06 & 0.30 & 0.02 \\
\hline
\end{tabular}

Table 2.3 Event 44 broadband modal decomposition - upper band (200-325 Hz)

\begin{tabular}{|c|c|c|c|c|c|c|}
\hline \multirow{2}{*}{ Mode } & \multicolumn{2}{|c|}{ All Regimes } & \multicolumn{2}{c|}{$\begin{array}{c}\text { Refraction } \\
\text { Regime }\end{array}$} & \multicolumn{2}{c|}{$\begin{array}{c}\text { NIW } \\
\text { Interference }\end{array}$} \\
\cline { 2 - 7 } & $\hat{\mu}$ & $\hat{\sigma}^{2}$ & $\hat{\mu}$ & $\hat{\sigma}^{2}$ & $\hat{\mu}$ & $\hat{\sigma}^{2}$ \\
\hline 1 & 0.66 & 0.33 & 0.50 & 0.19 & 0.69 & 0.29 \\
\hline 2 & 0.82 & 0.15 & 0.72 & 0.06 & 0.85 & 0.19 \\
\hline 3 & 1.14 & 0.34 & 1.10 & 0.15 & 1.13 & 0.40 \\
\hline 4 & 1.19 & 0.25 & 1.22 & 0.16 & 1.15 & 0.26 \\
\hline 5 & 1.18 & 0.28 & 1.22 & 0.17 & 1.12 & 0.25 \\
\hline
\end{tabular}




\section{B. Comments upon Event 47}

Figure 2.10 shows $I_{\tau}$ arrivals at the receiving array during Event 47 and at the same timescale as SW32 measurements. As in Event 44, a ramping of energy occurs prior to the NIW's arrival, between 22:40 and 22:55 GMT, again with seesawing energy spikes which can perhaps be explained by a Lloyd's Mirror interference pattern in the horizontal plane. Another possibility is that these fluctuations are simply due to small water column perturbations prior to the NIW's arrival, which are evident in the SW32 measurements. After a break in data, slightly erratic fluctuations are followed by a stark suppression of energy, which transpired concurrently with soliton activity along the acoustic track, between 23:20 and 23:25 GMT. This portion of data may be a defocusing event where energy is spread away from the receiving VLA. A subsequent brief collection of high energy signals could be attributed to some form of focusing, directing energy towards the VLA. After a second break in data, a longer period of reduced energy appears after 23:35 GMT. These data may be influenced by a shadow zone, where acoustic energy is shielded from the VLA behind the passing solitons - explained by the fact that the angle difference between the acoustic track and the NIW caused leading solitons to arrive at the source before the receiver. Alternatively, the NIW activity may have sufficiently passed the acoustic track, returning the acoustic field to a quiescent state.

\subsection{SUMMARY AND CONCLUSIONS}

In brief summary, measured data from the SW06 experiment exhibit general features that can be characterized by a time-varying scenario where the acoustic track is nearly parallel to an approaching NIW train. Local oceanographic measurements confirm strong internal wave activity during two discrete NIW events. Given our experimental 
geometry, horizontal refraction effects dominate, as shown by theory and threedimensional modeling. Further insight into the measured data is offered through the use of broadband energy metrics, modal decomposition, and statistical analysis. A suggested breakdown of the data based upon time-varying regimes is offered through the interpretation of observational data.

In conclusion, this work offers good corroboration with previous studies. Future work will better establish the suggested time-varying scenario. This includes examining other SW06 acoustic data during similar experimental configurations, and also simulating the NIW with three-dimensional parabolic equation modeling. 


\section{ACKNOWLEDGEMENTS}

The authors are indebted to the dedicated and hard-working participants of the SW06 experiment, with special thanks to the WHOI mooring crews and personnel aboard the R/V Sharp. The accommodating technical discussions and help from Art Newhall, Ying Tsong Lin, Glen Gawarkiewicz, Donglai Gong, Emily Shroyer (WHOI) and Steve Crocker (Univ. of Rhode Island) were incredibly helpful for this work. Our deepest gratitude is extended to ONR who funded the SW06 experiment, and the exciting research that has followed. 


\section{REFERENCES AND LINKS}

1. C. W. Ufford, "Internal waves off San Diego, California," University of California Division of War Research Reports (1945).

2. O. S. Lee, "Effect of an Internal Wave on Sound in the Ocean," J. Acoust. Soc. Am. 33 (5), 677-681 (1961).

3. I. Dyer, "Statistics of Sound Propagation in the Ocean," J. Acoust. Soc. Am. 48 (1B), 337-345 (1970).

4. W. H. Munk and F. Zachariasen, "Sound propagation through a fluctuating stratified ocean: Theory and observation," J. Acoust. Soc. Am. 59 (4), 818-838 (1976).

5. F. Dyson, W. Munk and B. Zetler, "Interpretation of multipath scintillations Eleuthera to Bermuda in terms of internal waves and tides," J. Acoust. Soc. Am. 59 (5), 1121-1133 (1976).

6. S. M. Flatte, R. Dashen, W. H. Munk, K. M. Watson and F. Zachariasen, Sound transmission through a fluctuating ocean. (Cambridge University Press, Cambridge, 1979).

7. J.-X. Zhou, X.-z. Zhang and P. H. Rogers, "Resonant interaction of sound wave with internal solitons in the coastal zone," J. Acoust. Soc. Am. 90 (4), 2042-2054 (1991).

8. M. Badiey and P. H. Rogers, "Acoustical Oceanography and Underwater Acoustics: Ocean Acoustics in Shallow Water: What are the Issues? Special Sessions I-III," J. Acoust. Soc. Am. 93 (4), 2267-2286 (1993).

9. B. J. Sperry, J. F. Lynch, G. Gawarkiewicz, C.-S. Chiu and A. Newhall, "Characteristics of acoustic propagation to the eastern vertical line array receiver during the summer 1996 New England shelfbreak PRIMER experiment," IEEE J. Ocean. Eng. 28 (4), 729-749 (2003).

10. J. R. Apel, M. Badiey, C.-S. Chiu, S. Finette, R. Headrick, J. Kemp, J. F. Lynch, A. Newhall, M. H. Orr, B. H. Pasewark, D. Tielbuerger, A. Turgut, K. Von Der 
Heydt and S. Wolf, "An overview of the 1995 SWARM shallow-water internal wave acoustic scattering experiment," IEEE J. Ocean. Eng. 22 (3), 465-500 (1997).

11. J. F. Lynch, S. R. Ramp, C.-S. Chiu, T. Y. Tang, Y. J. Yang and J. A. Simmen, "Research highlights from the Asian Seas International Acoustics Experiment in the South China Sea," IEEE J. Ocean. Eng. 29 (4), 1067-1074 (2004).

12. P. H. Dahl, R. Zhang, J. H. Miller, L. R. Bartek, Z. Peng, S. R. Ramp, J. X. Zhou, C. S. Chiu, J. F. Lynch, J. A. Simmen and others, "Overview of results from the asian seas international acoustics experiment in the east china sea," IEEE J. Ocean. Eng. 29 (4), 920-928 (2005).

13. A. E. Newhall, T. F. Duda, K. Von Der Heydt, J. D. Irish, J. N. Kemp, S. A. Lerner, S. P. Liberatore, Y. T. Lin, J. F. Lynch, A. R. Maffei and others, "Acoustic and oceanographic observations and configuration information for the WHOI moorings from the SW06 experiment," WHOI technical report No. WHOI-2007-04 (2007).

14. M. Badiey, B. G. Katsnelson, J. F. Lynch and S. Pereselkov, "Frequency dependence and intensity fluctuations due to shallow water internal waves," J. Acoust. Soc. Am. 122 (2), 747-760 (2007).

15. M. Badiey, I. Jaya and A. H. Cheng, "Shallow-water acoustic/geoacoustic experiments at the New Jersey Atlantic Generating Station site," J. Acoust. Soc. Am. 96 (6), 3593-3604 (1994).

16. T. F. Duda, "Acoustic mode coupling by nonlinear internal wave packets in a shelfbreak front area," IEEE J. Ocean. Eng. 29 (1), 118-125 (2004).

17. H. Weinberg and R. Burridge, "Horizontal ray theory for ocean acoustics," J. Acoust. Soc. Am. 55 (1), 63-79 (1974).

18. Y. A. Kravtsov, V. M. Kuzkin and V. G. Petnikov, "Perturbation calculation of the horizontal refraction of sound waves in a shallow sea," Sov. Phys. Acoust. 30, 45-47 (1984). 
19. R. Oba and S. Finette, "Acoustic propagation through anisotropic internal wave fields: Transmission loss, cross-range coherence, and horizontal refraction," J. Acoust. Soc. Am. 111 (2), 769-784 (2002).

20. B. G. Katsnelson and S. A. Pereselkov, "Low-Frequency Horizontal Acoustic Refraction Caused by Internal Wave Solitons in a Shallow Sea.," Acoust. Phys. 46 (6), 684 (2000).

21. M. Badiey, Y. Mu, J. Lynch, J. Apel and S. Wolf, "Temporal and azimuthal dependence of sound propagation in shallow water with internal waves," IEEE J. Ocean. Eng. 27 (1), 117-129 (2002).

22. B. G. Katsnelson, S. A. Pereselkov, V. G. Petnikov, K. D. Sabinin and A. N. Serebryanyi, "Acoustic Effects Caused by High-Intensity Internal Waves in a Shelf Zone.," Acoust. Phys. 47 (4), 424 (2001).

23. M. Badiey, B. G. Katsnelson, J. F. Lynch, S. Pereselkov and W. L. Siegmann, "Measurement and modeling of three-dimensional sound intensity variations due to shallow-water internal waves," J. Acoust. Soc. Am. 117 (2), 613-625 (2005).

24. S. D. Frank, M. Badiey, J. F. Lynch and W. L. Siegmann, "Analysis and modeling of broadband airgun data influenced by nonlinear internal waves," J. Acoust. Soc. Am. 116 (6), 3404-3422 (2004).

25. S. D. Frank, M. Badiey, J. F. Lynch and W. L. Siegmann, "Experimental evidence of three-dimensional acoustic propagation caused by nonlinear internal waves," J. Acoust. Soc. Am. 118 (2), 723-734 (2005).

26. D. B. Reeder, T. F. Duda and B. Ma, "Short-Range Acoustic Propagation Variability on a Shelf Area with Strong Nonlinear Internal Waves," (2008).

27. J. Luo, M. Badiey, E. A. Karjadi, B. Katsnelson, A. Tskhoidze, J. F. Lynch and J. N. Moum, "Observation of sound focusing and defocusing due to propagating nonlinear internal waves," J. Acoust. Soc. Am. 124 (3), 66 (2008).

28. Y.-T. Lin, T. F. Duda and J. F. Lynch, "Acoustic mode radiation from the termination of a truncated nonlinear internal gravity wave duct in a shallow ocean area," J. Acoust. Soc. Am. 126 (4), 1752-1765 (2009). 
29. J. F. Lynch, Y. T. Lin, T. F. Duda and A. E. Newhall, "Acoustic Ducting, Reflection, Refraction, and Dispersion by Curved Nonlinear Internal Waves in Shallow Water," IEEE J. Ocean. Eng. 35 (1), 12-27 (2010).

30. J. F. Lynch, J. A. Colosi, G. G. Gawarkiewicz, T. F. Duda, A. D. Pierce, M. Badiey, B. G. Katsnelson, J. E. Miller, W. Siegmann, C.-S. Chiu and A. Newhall, "Consideration of fine-scale coastal oceanography and 3-D acoustics effects for the ESME sound exposure model," IEEE J. Ocean. Eng. 31 (1), 33-48 (2006).

31. J. R. Apel, "A New Analytical Model for Internal Solitons in the Ocean.," Journal of Physical Oceanography 33 (11), 2247 (2003).

32. S. Finette, M. H. Orr, A. Turgut, J. R. Apel, M. Badiey, C.-s. Chiu, R. H. Headrick, J. N. Kemp, J. F. Lynch, A. E. Newhall, K. v. Heydt, B. Pasewark, S. N. Wolf and D. Tielbuerger, "Acoustic field variability induced by time evolving internal wave fields," J. Acoust. Soc. Am. 108 (3), 957-972 (2000).

33. R. R. Carhart, "Lloyd Mirror Effect in a Variable Velocity Medium," University of California Division of War Research Reports (1943).

34. M. Badiey, B. G. Katsnelson, Y. T. Lin and J. F. Lynch, "Acoustic multipath arrivals in the horizontal plane due to approaching nonlinear internal waves," J. Acoust. Soc. Am. 129 (4) (2011).

35. M. Badiey, B. Katsnelson, J. Luo, L. Brown, J. Yang, G. A. Dossot, S. E. Crocker, J. Seigel, J. Largeaud and L. Wan, "R/V Sharp SW06 Cruise Technical Report," University of Delaware Technical Report (2010).

36. D. Gong, J. T. Kohut and S. M. Glenn, "Seasonal climatology of wind-driven circulation on the New Jersey Shelf," J. Geophys. Res. 115 (C4), 04006 (2010).

37. E. L. Shroyer, "Nonlinear Internal Waves on the Continental Shelf," Ph.D. Dissertation, Col. Oceanic Atmos. Sci., Oregon State Univ., Corvallis, OR, Sep. 2009.

38. Q. Li, D. M. Farmer, T. F. Duda and S. Ramp, "Acoustical Measurement of Nonlinear Internal Waves Using the Inverted Echo Sounder," J. Atmos. Oceanic Technol. 26 (10), 2228-2242 (2009). 
39. K. V. Mackenzie, "Nine-term equation for sound speed in the oceans," J. Acoust. Soc. Am. 70 (3), 807-812 (1981).

40. T. F. Duda, J. F. Lynch, A. E. Newhall, L. Wu and C.-S. Chiu, "Fluctuation of 400-Hz sound intensity in the 2001 ASIAEX South China Sea experiment," IEEE J. Ocean. Eng. 29 (4), 1264-1279 (2004).

41. A. Fredricks, J. A. Colosi, J. F. Lynch, G. Gawarkiewicz, C.-S. Chiu and P. Abbot, "Analysis of multipath scintillations from long range acoustic transmissions on the New England continental slope and shelf," J. Acoust. Soc. Am. 117 (3), 1038-1057 (2005).

42. S. E. Crocker, J. H. Miller, G. R. Potty and J. F. Lynch, "Nonlinear optimization for beamforming a geometrically deficient vertical line array: Application to sediment tomography," J. Acoust. Soc. Am. 120 (5), 3063-3063 (2006).

43. M. B. Porter, "The KRAKEN normal mode program," Technical report, SACLANT Undersea Research Center (1992).

44. Y.-M. Jiang, N. R. Chapman and M. Badiey, "Quantifying the uncertainty of geoacoustic parameter estimates for the New Jersey shelf by inverting air gun data," J. Acoust. Soc. Am. 121 (4), 1879-1894 (2007). 


\section{FIGURES}
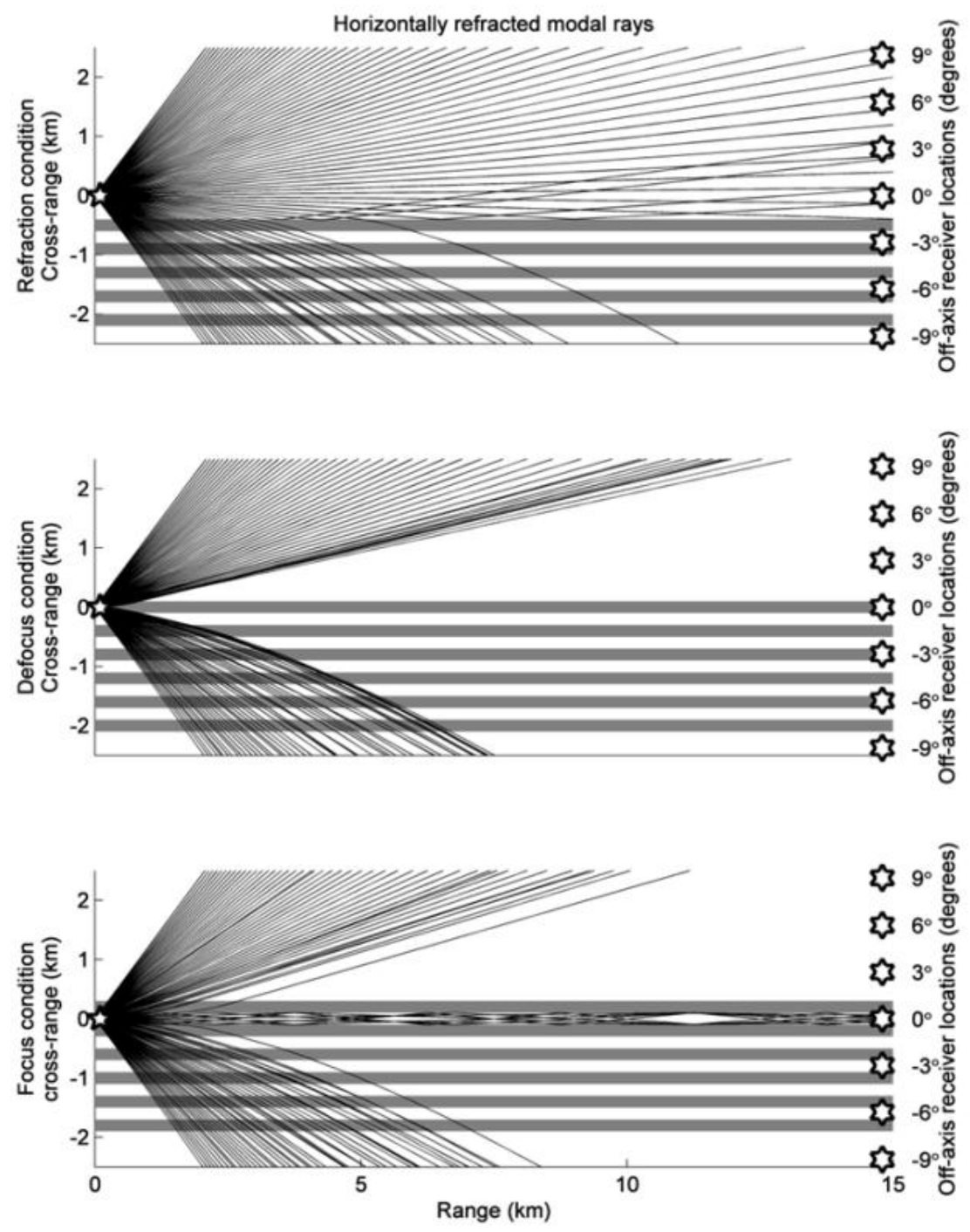

Figure 2.1 The NIW train can be approximated as series of "sudden" perturbations in the water column, where each soliton alters the modal eigenvalues. Depending on the source-receiver position, the NIW can heavily influence where acoustic energy is directed. The top panel shows a refraction scenario - prior to the NIW reaching the acoustic source. The center panel shows a defocusing scenario - just as the first soliton reaches the source. The bottom panel shows a focusing scenario - as the source is trapped between two solitons. Soliton perturbations are represented as gray bars for visual interpretation. 


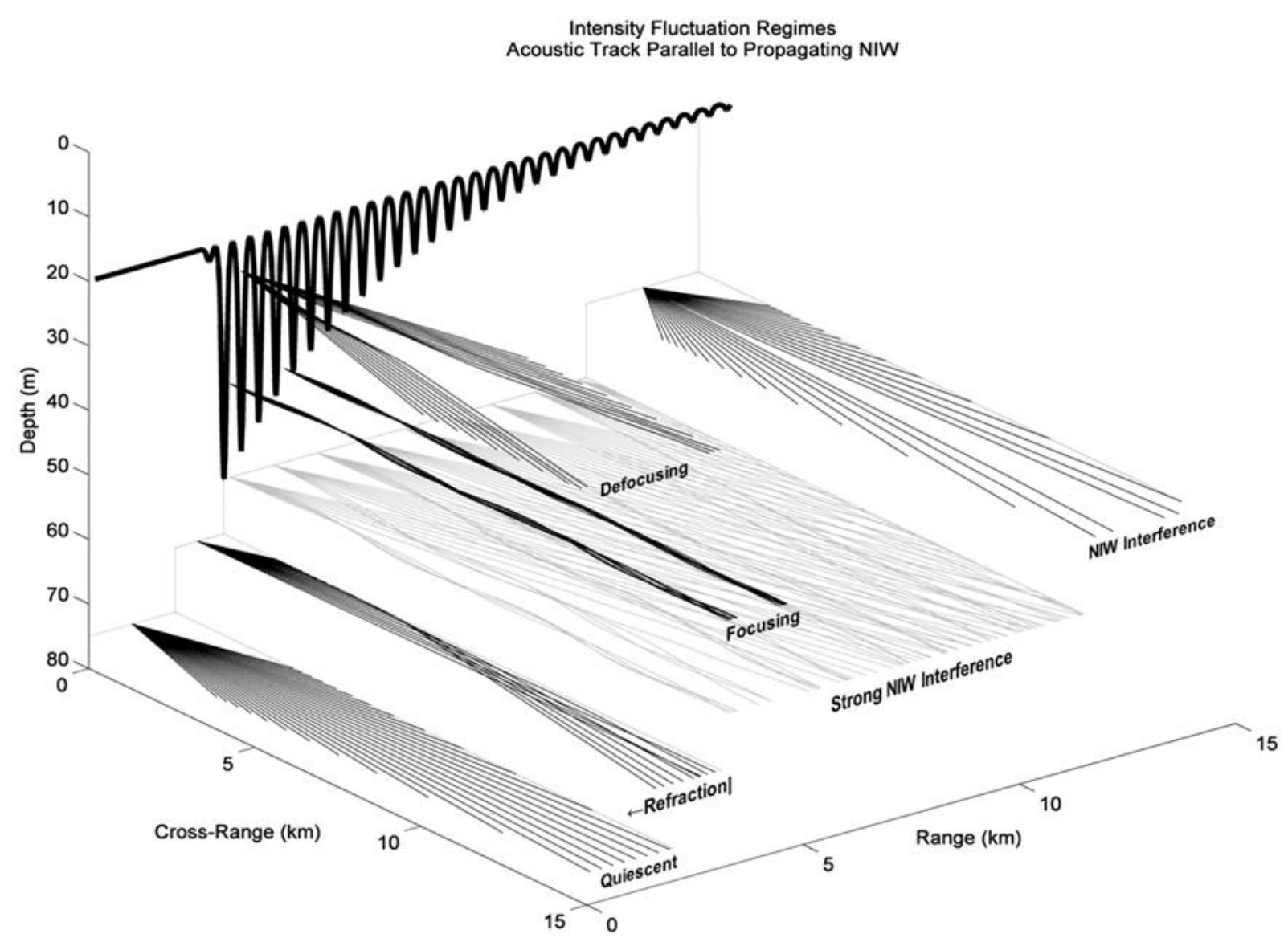

Figure 2.2 Time varying intensity fluctuation regimes for a propagating NIW field passing an acoustic source. An acoustic source emits horizontally refracted modal rays at frequency $f$ and mode number $M$. Note: Rays are not depthdependent, and are plotted at different levels to clarify different regimes. A quiescent regime is followed by a refraction regime prior to the NIW's arrival. As the NIW passes over an acoustic source both focusing and defocusing cause a complicated interference regime. 


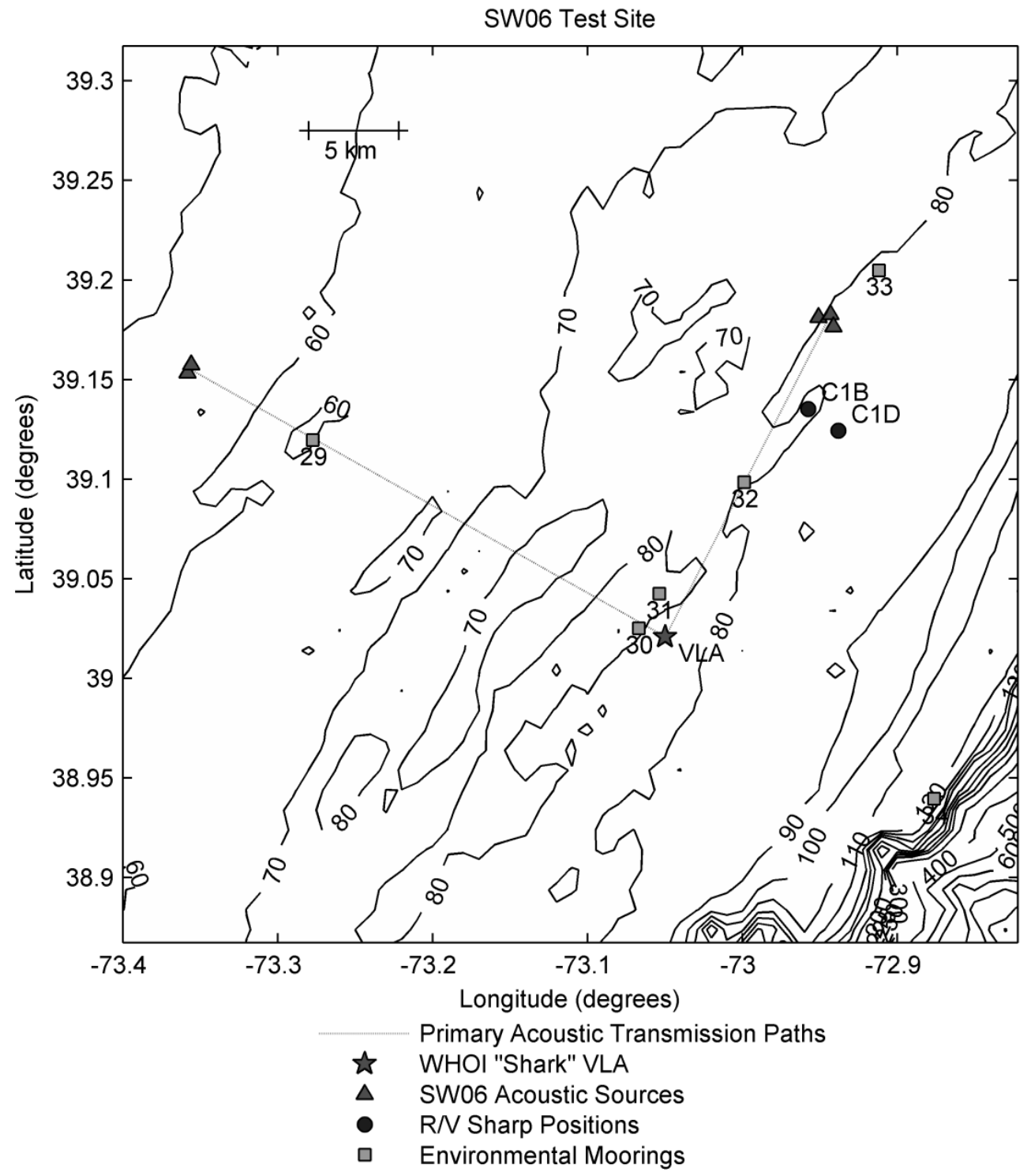

Figure 2.3 Map of the SW06 experiment, and locations of the R/V Sharp during the two specific NIW Events 44 and 47 (C1B and C1D respectively) 

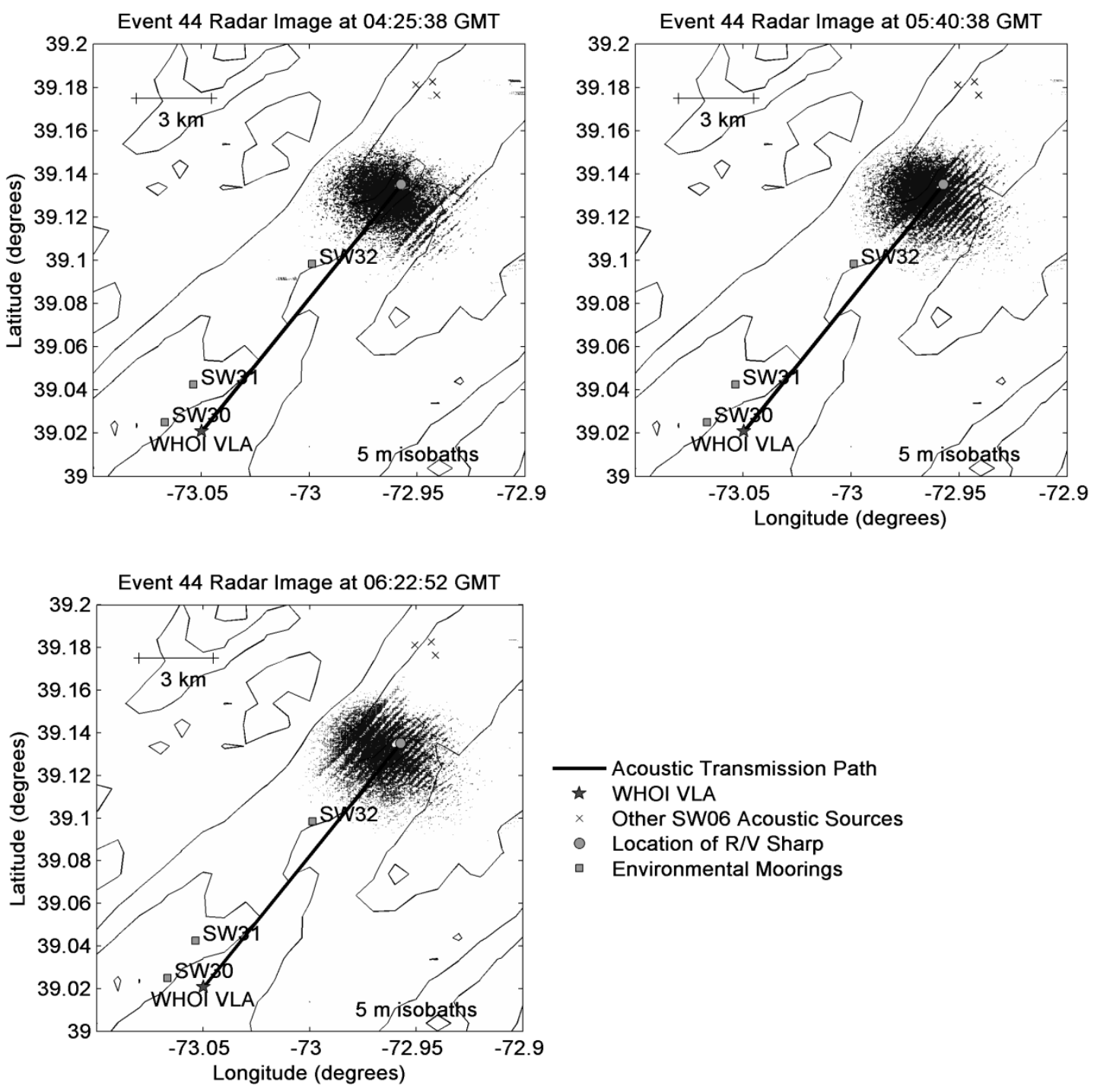

Figure 2.4 A strong internal wave event propagated nearly parallel to the sourcereceiver acoustic track (R/V Sharp J-15 and WHOI VLA respectively). Ship radar detected the surface expression of the NIW as it approached, passed beneath, and left the $R / V$ Sharp. 
Event 44 - Normalized Echo Backscatter Starting at 14-Aug-2006 02:47:34

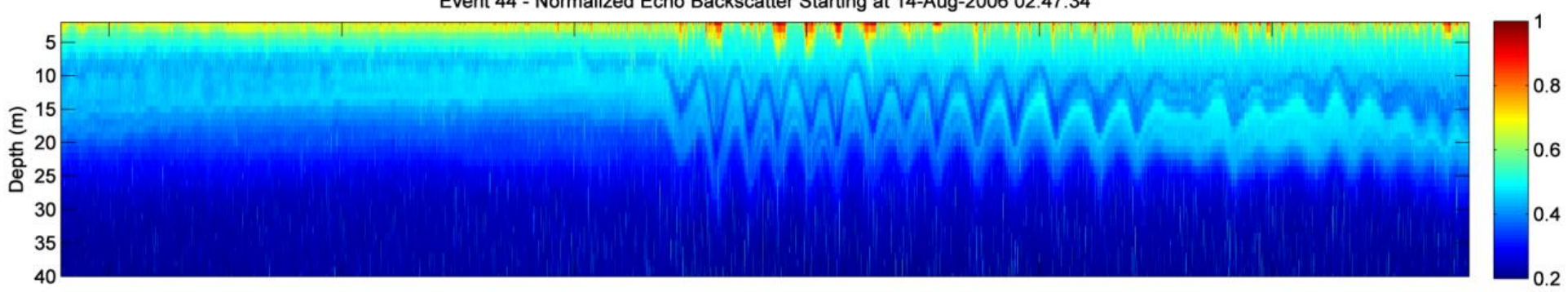

Velocity Magnitude $(\mathrm{cm} / \mathrm{s})$
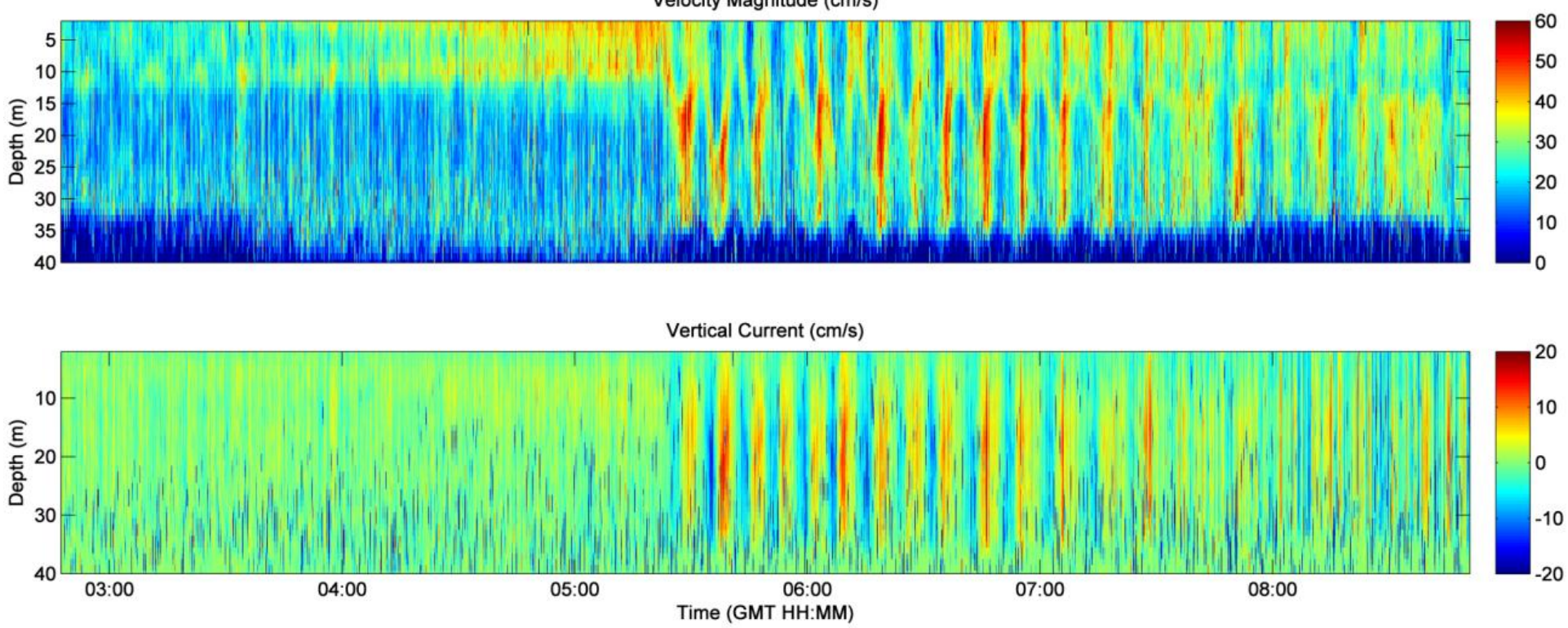

Figure 2.5 ADCP plot of Event 44. A strong NIW passed below the R/V Sharp on Aug 14 2006. Top panel shows normalized echo backscatter, showing strong pycnocline depressions occurring between 05:30 and 07:30 GMT. Center panel shows the magnitude of vertical particle velocity. Bottom panel shows vertical velocity. (color online) 

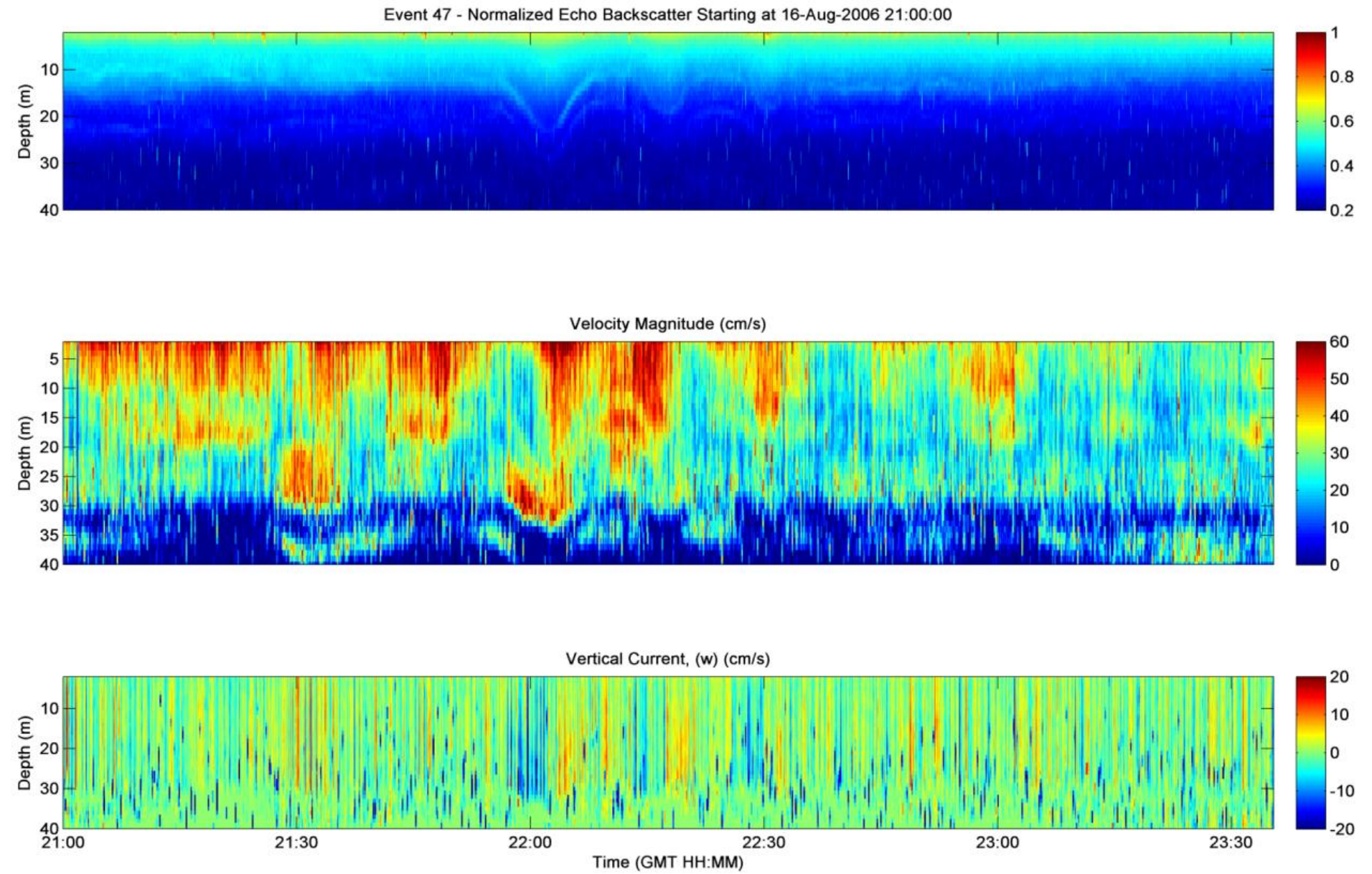

Figure 2.6 ADCP plot of Event 47. A strong NIW passed below the R/V Sharp on Aug 16 2006. Top panel shows normalized echo backscatter, showing three strong pycnocline depressions. Center panel shows the magnitude of vertical particle velocity. Bottom panel shows vertical velocity. (color online) 

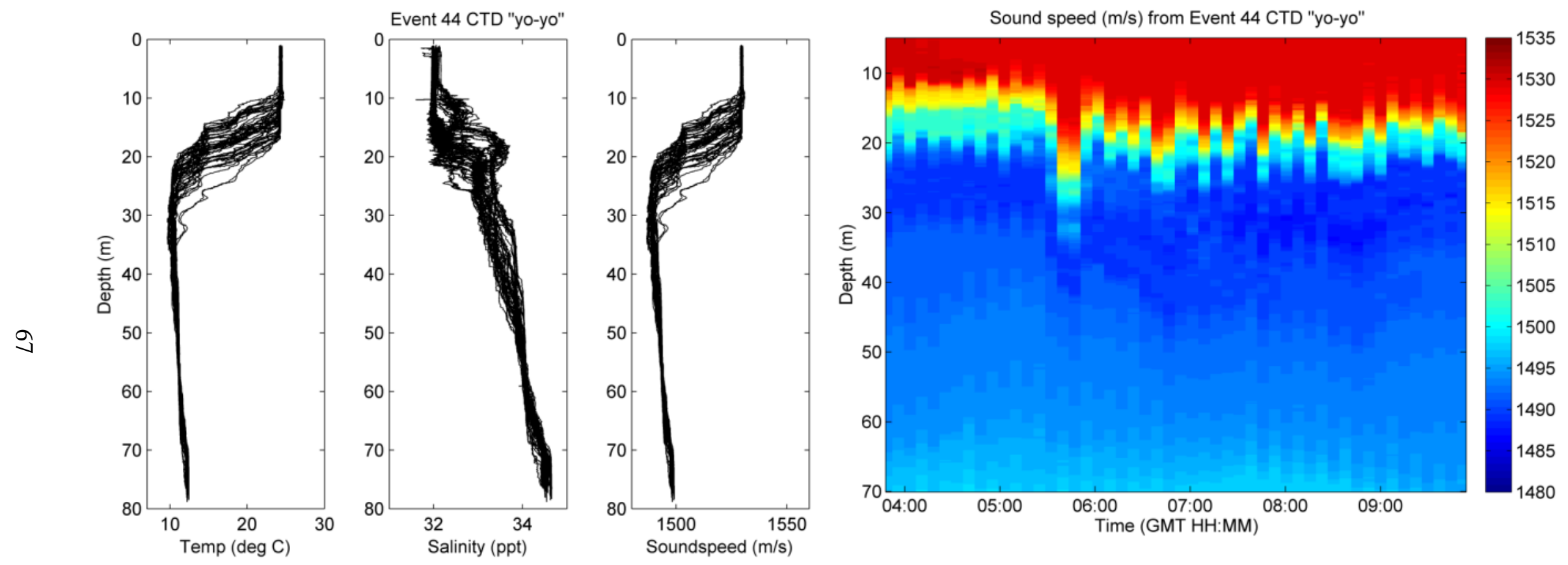

Figure 2.7 Computed sound speed versus time during CTD "yo-yo." Event 44 arrives at the ship and acoustic source at roughly 05:40 GMT and is marked by a depressed region of faster sound speed, followed by fluctuating sound speed profiles versus time and depth. (color online) 

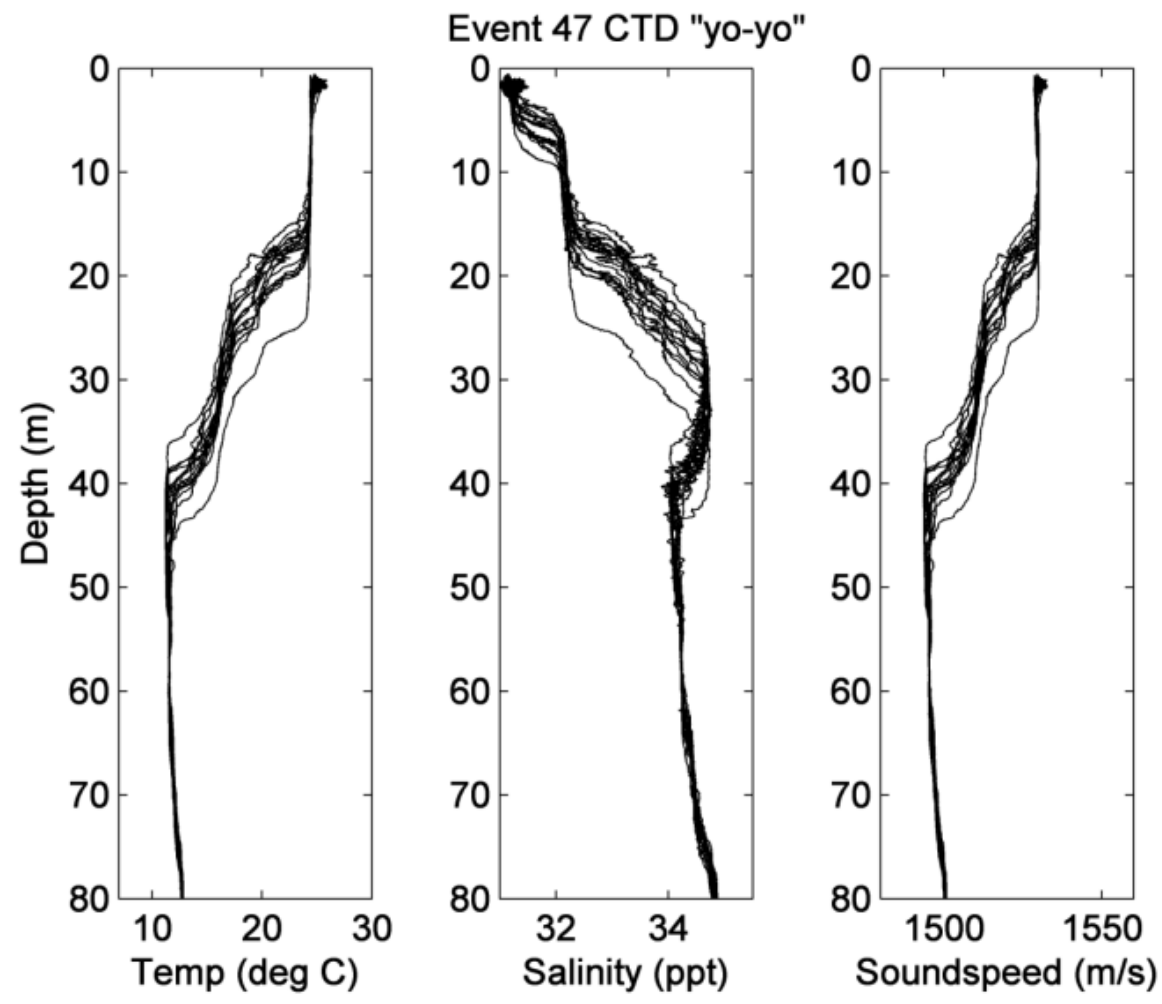

Figure 2.8 CTD measurements of temperature (left), salinity (center), and computed sound speed (right) during event 47. 

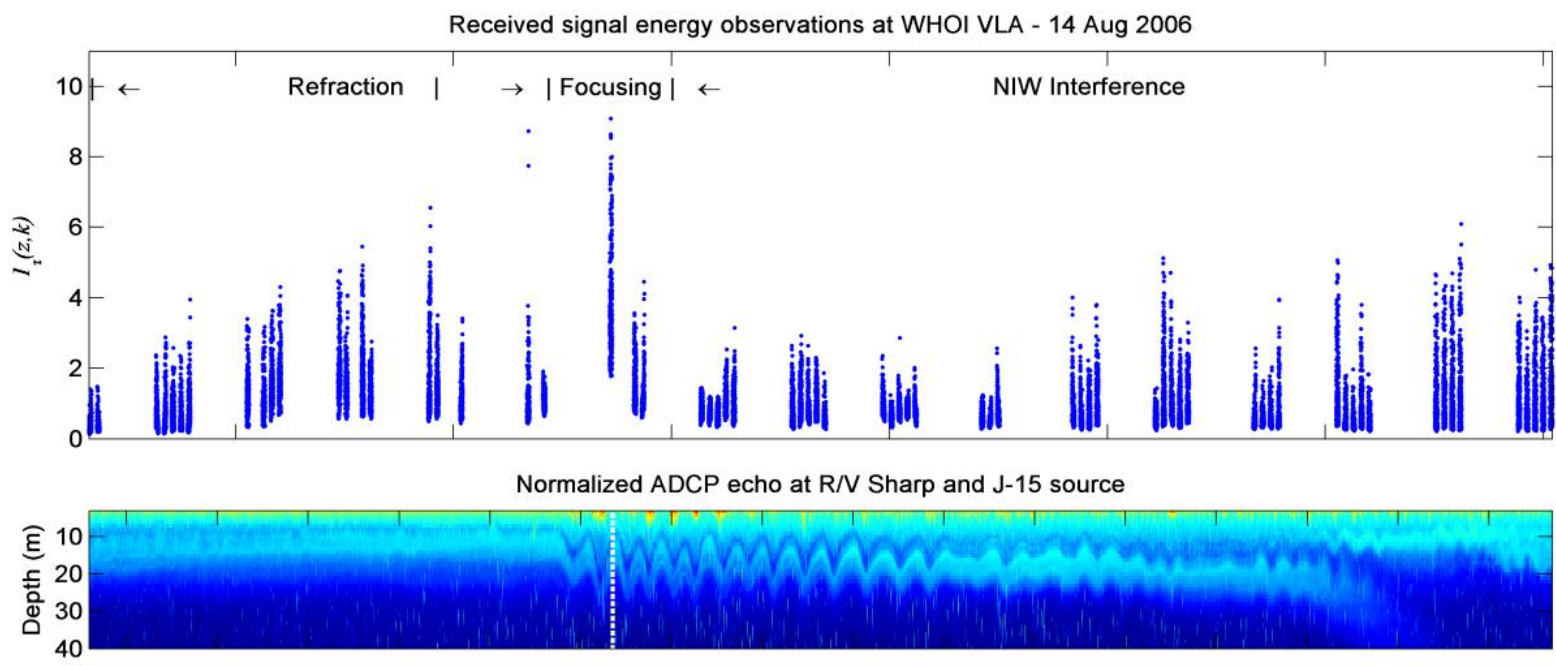

Sound speed at Environmental Mooring SW32

$\mathrm{C}(\mathrm{m} / \mathrm{s})$

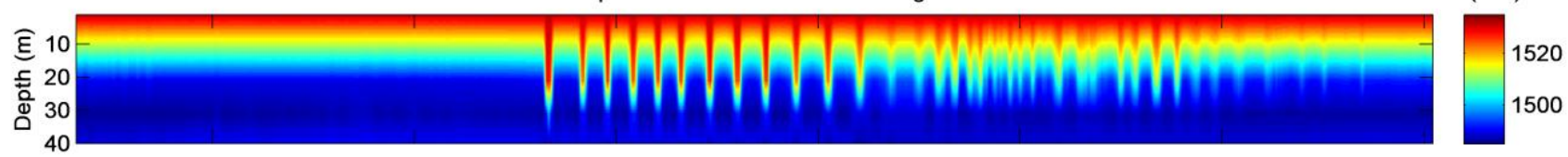

Sound speed at WHOI VLA

$\mathrm{C}(\mathrm{m} / \mathrm{s})$

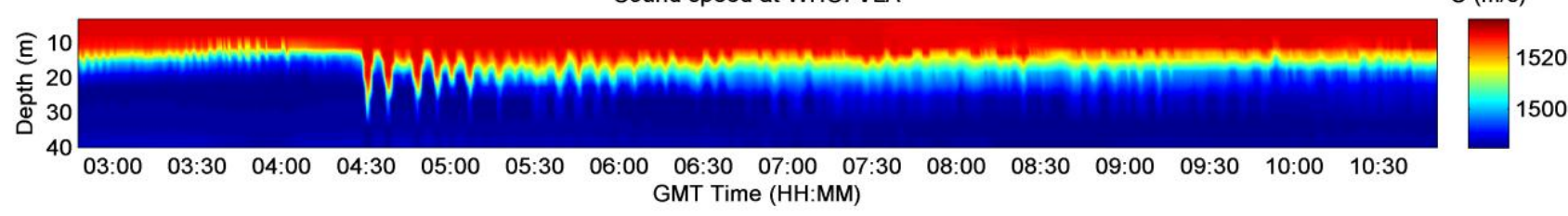

Figure 2.9 (Top) Measured normalized energy levels received upon each hydrophone of the WHOI VLA versus time. Refraction occurs prior to the NIW's arrival, with possible focusing as the NIW arrives at the acoustic source. A complicated NIW interference regime follows; (Bottom 3 plots) Environmental sensors including R/V Sharp ADCP (at the acoustic source) showing NIW pycnocline, mooring SW32 (midway between source-receiver), and interpolated sound speed at the WHOI receiving VLA, all exhibiting strong internal wave activity. (color online) 

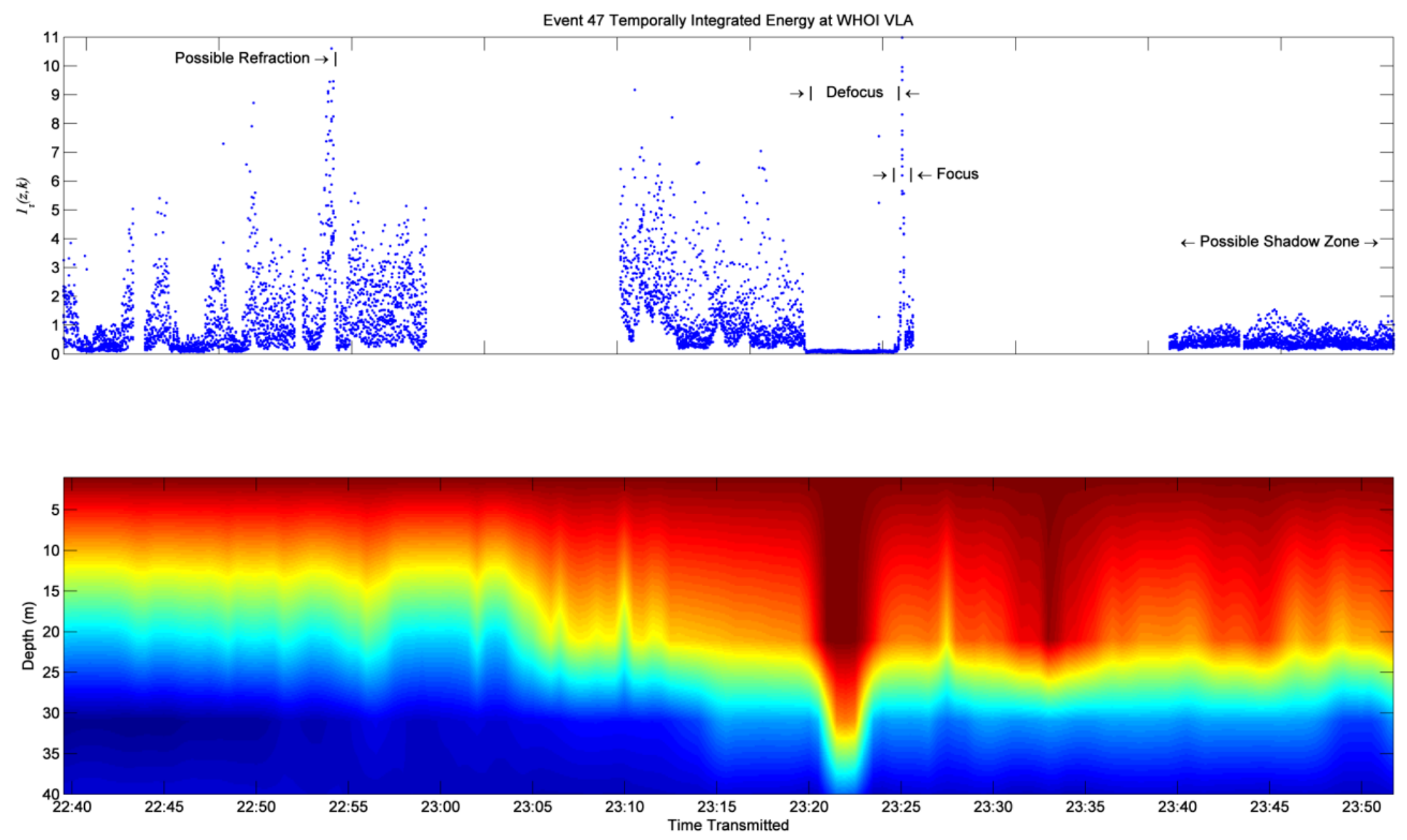

Figure 2.10 Measured normalized energy levels received upon each hydrophone of the WHOI VLA versus time during Event 47. Possible refraction occurs prior to the NIW's arrival, with defocusing and focusing effects as the soliton reaches the acoustic track, and a possible shadow zone following (top). Environmental mooring SW32 was situated midway between the source and receiver and shows evidence of strong soliton activity (bottom). (color online) 

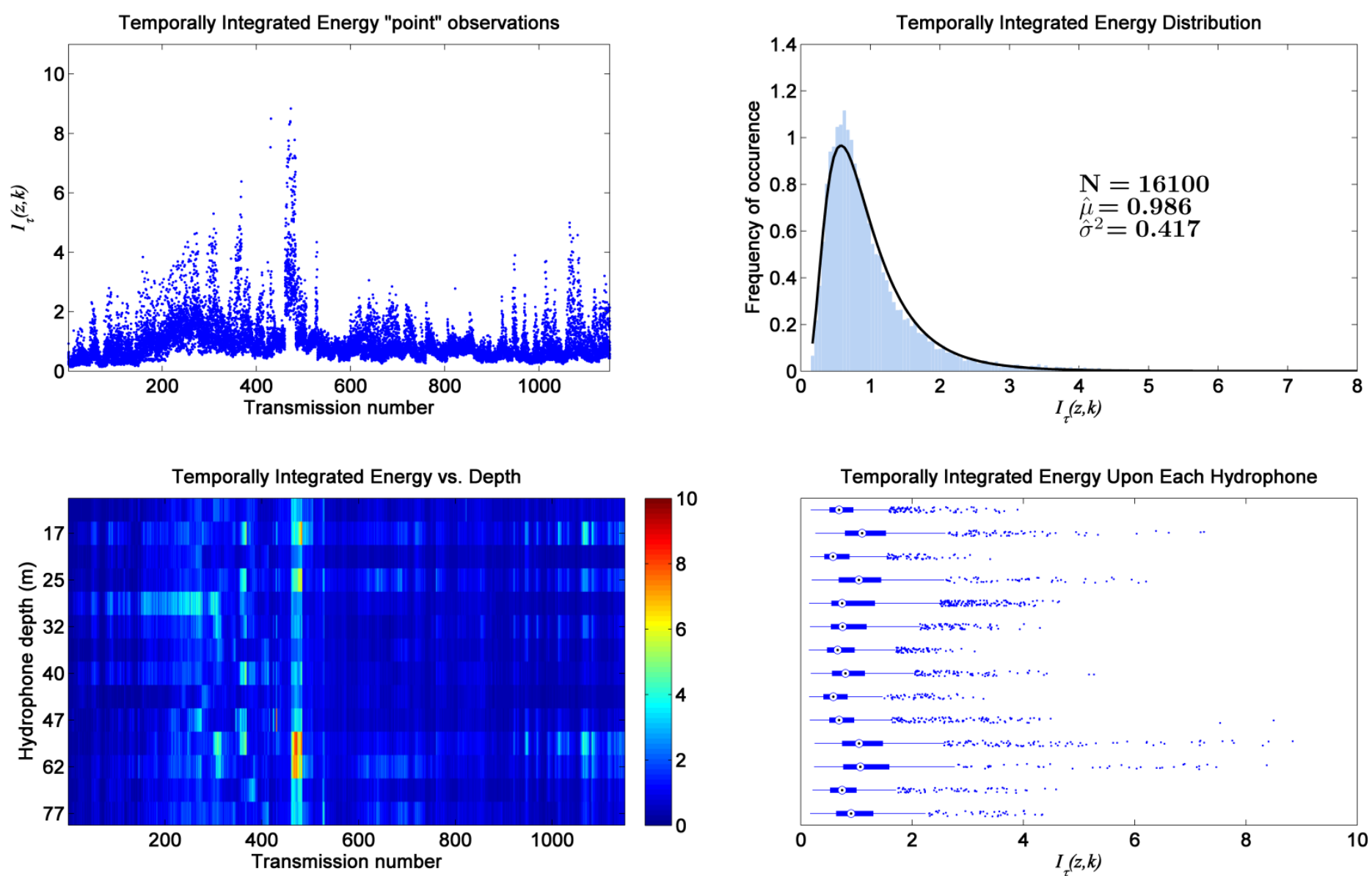

Figure 2.11 Point observations are plotted versus transmission number (top left), and the data fits well to a lognormal distribution (top right). Depth dependent variability is plotted using a normalized color scalogram versus transmission number (bottom left), or alternatively shown through a statistical boxplot of received energy upon each hydrophone (bottom right). (color online) 

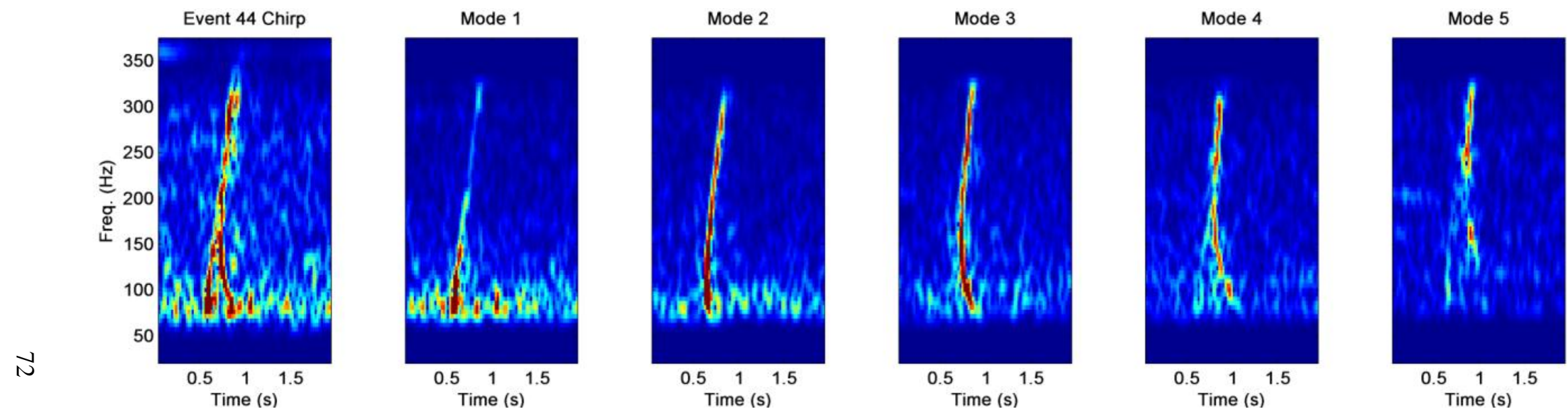

Figure 2.12 Spectrogram showing a received chirp signal upon the eighth hydrophone (at 39 meters deep) of the WHOI VLA (left). This signal is broken down into broadband modal components for modes 1-5 (right panels). Note the "splitting" of the signal can be explained by separate modal arrivals. Color scales across plots are identical. (color online) 

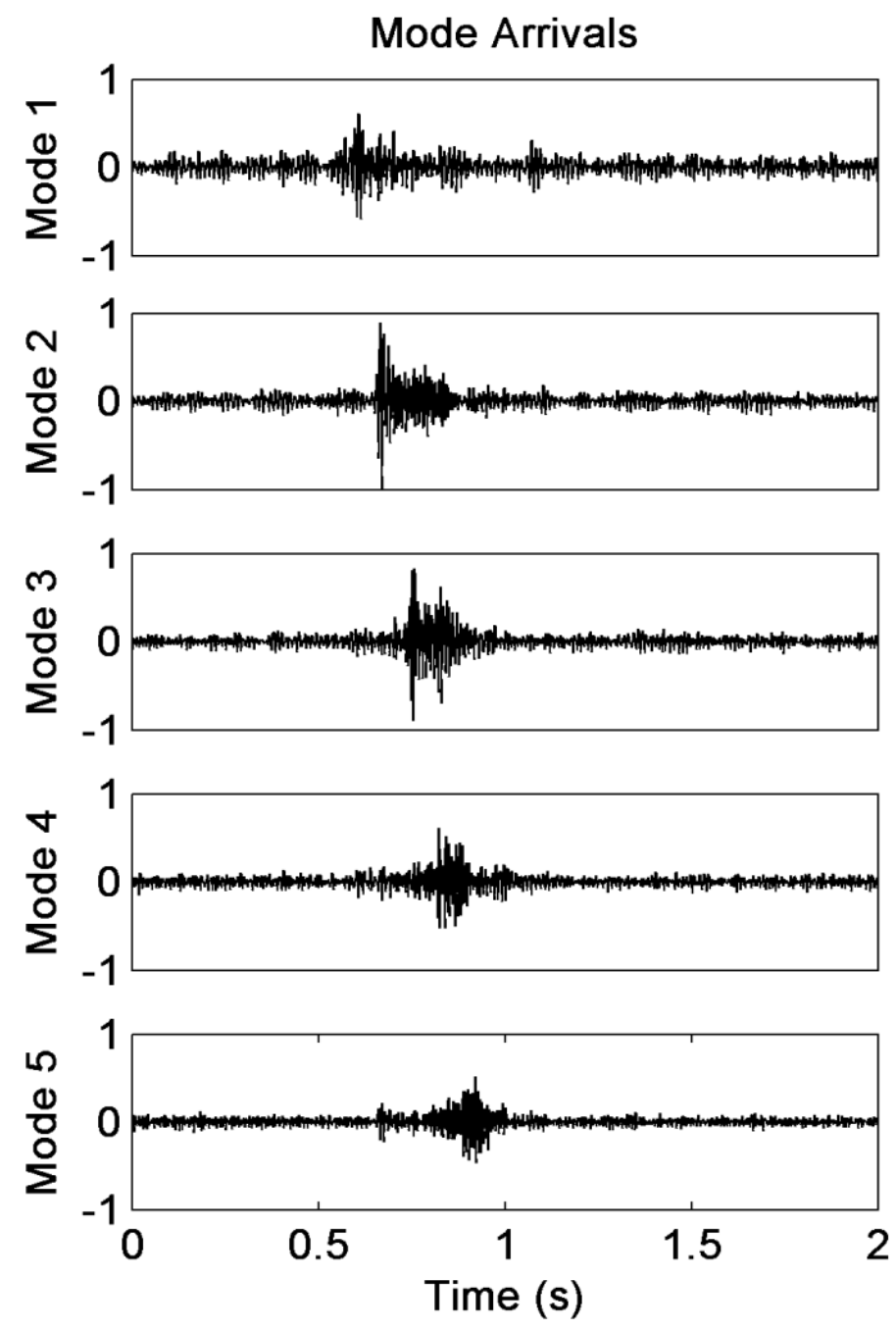

Figure 2.13 Normalized modal arrivals for the chirp signal shown in Figure 2.12 . 

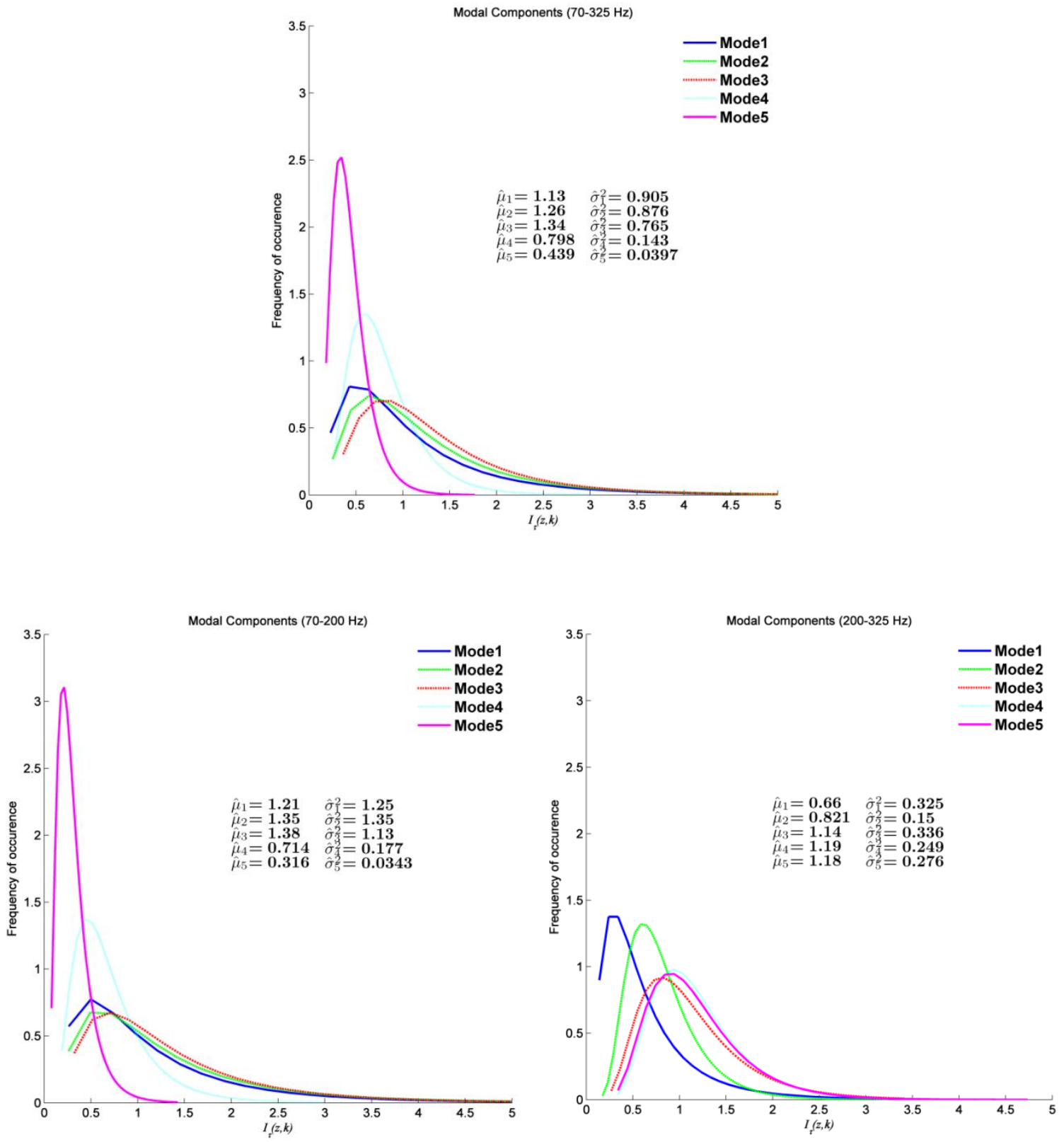

Figure 2.14 Modal components of intensity point observations for 70-325 $\mathrm{Hz}$ (left); the lower frequency spectrum, 70-200 $\mathrm{Hz}$ (center); and the upper frequency spectrum, 200-325 $\mathrm{Hz}$ (right). Modes 1-3 dominate energy in the lower frequency band, while modes 3-5 dominate energy in the upper frequency spectrum. (color online) 

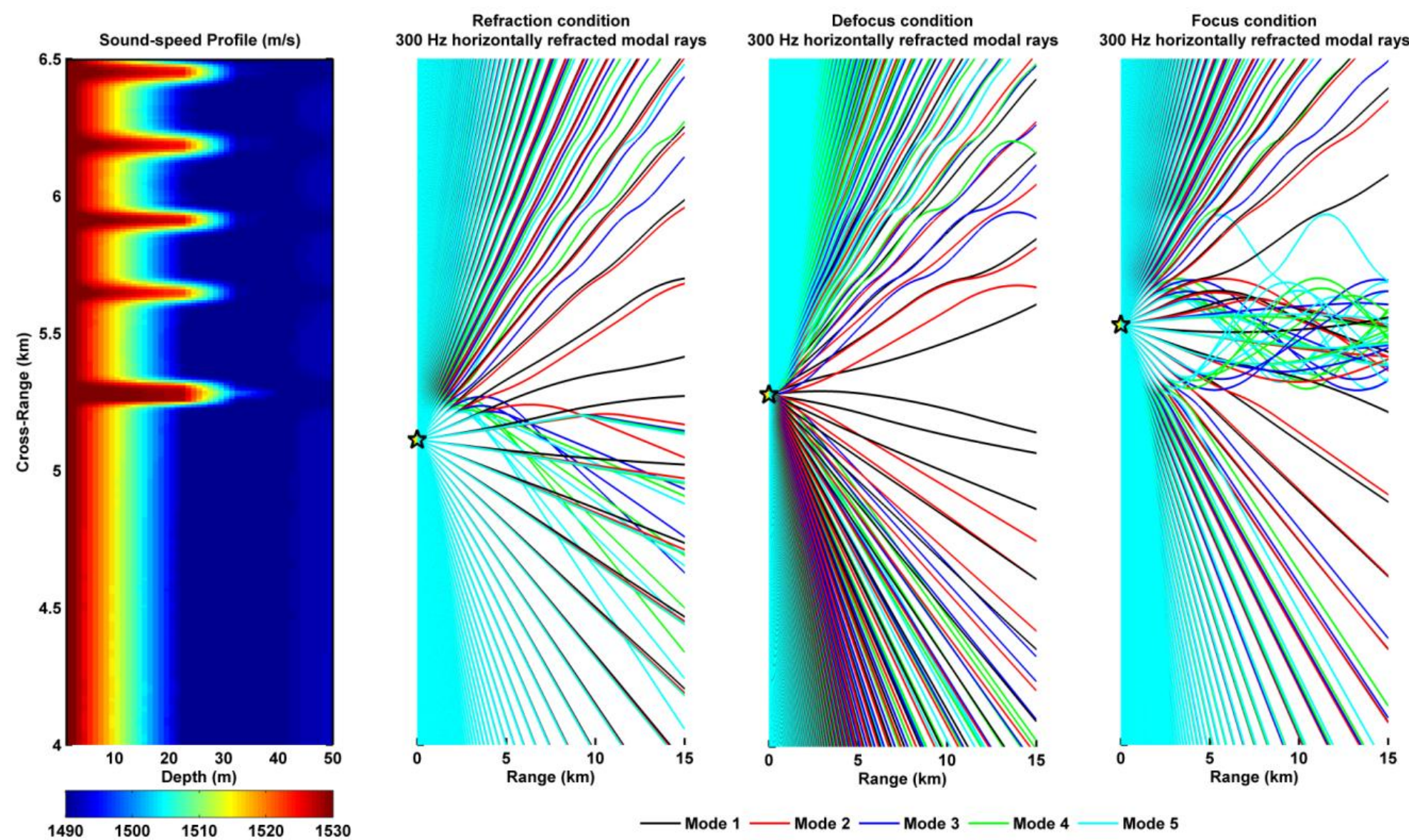

$\begin{array}{lllll}1490 & 1500 & 1510 & 1520 & 1530\end{array}$

- Mode $1-$ Mode $2-$ Mode $3-$ Mode $4 \longrightarrow$ Mode 5

Figure 2.15 Event 44 sound speed profile (left) used as the primary environmental variable in the 3D KRAKEN normal mode code, and is assumed to remain valid across range. The acoustic source is located at the star, simulating a passing NIW. Modal rays are horizontally refracted by the NIW in a refraction, defocus, and focus condition based on the position of the source. (color online) 


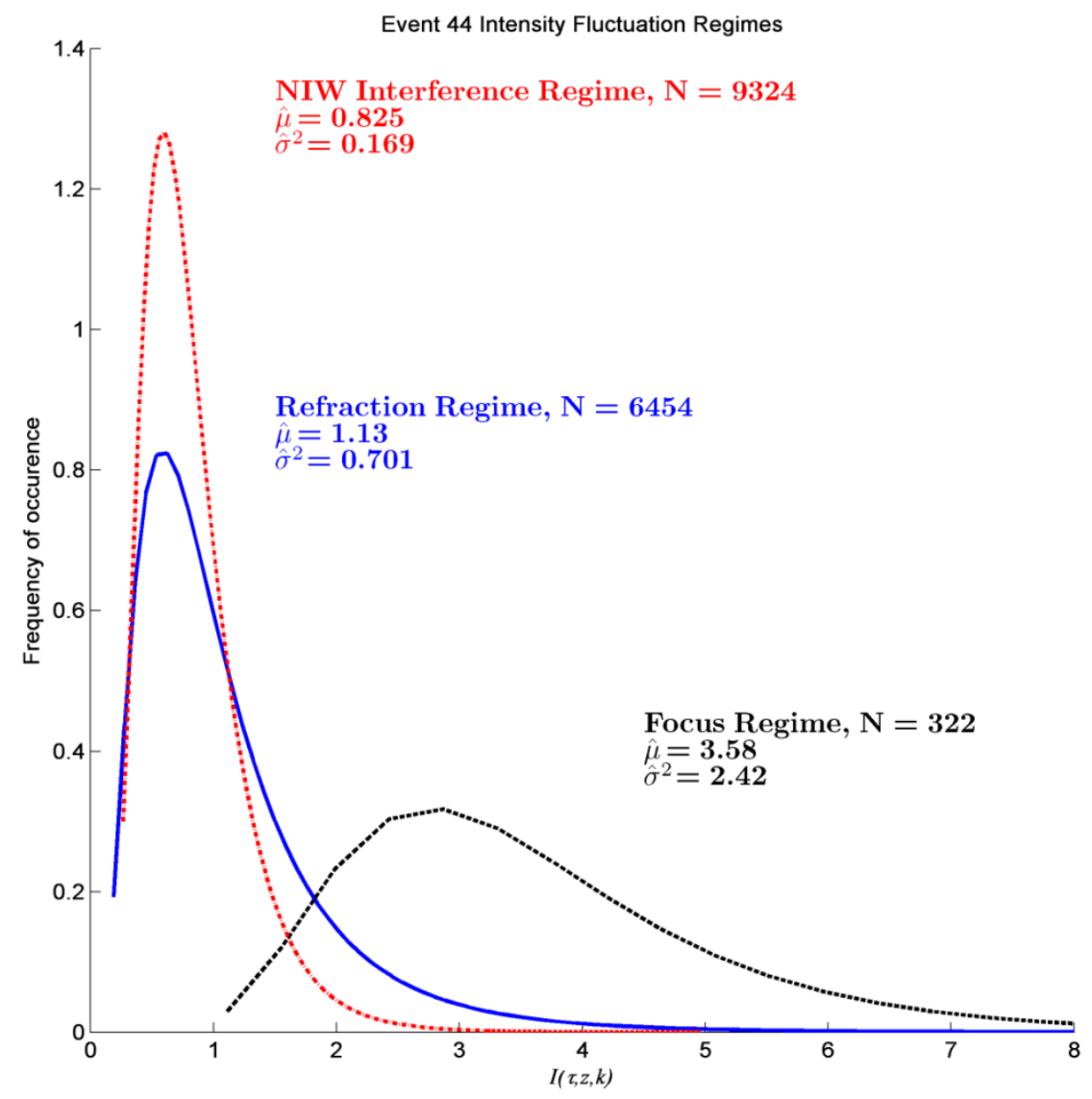

Figure 2.16 Event 44 is separated into the proposed refraction, focusing, and NIW interference regimes. Refraction effects prior to the NIW's arrival cause similar variability as NIW interference effects. Focusing effects dominate both intensification and signal variability. (color online) 


\section{MANUSCRIPT - III}

Intended for submission to the Journal of the Acoustical Society of America

\section{Examining energy fluctuations during strong internal wave activity using a three-dimensional parabolic} equation model

\section{Corresponding Author:}

Georges A. Dossot

Department of Ocean Engineering, University of Rhode Island, Narragansett, Rhode Island 02882, georges.dossot@gmail.com

\section{Contributing Authors:}

\section{Kevin B. Smith}

Department of Physics, Naval Postgraduate School, Monterey, California 93943

\section{James H. Miller, Gopu R. Potty}

Department of Ocean Engineering, University of Rhode Island, Narragansett, Rhode Island 02882

\section{Mohsen Badiey}

College of Marine and Earth Studies, University of Delaware, Newark, Delaware 19716

James F. Lynch

Department of Applied Ocean Physics and Engineering, Woods Hole Oceanographic Institution, Woods Hole, Massachusetts 02543 
Abstract: The three-dimensional Monterey-Miami Parabolic Equation (MMPE) model is used to simulate a nonlinear internal wave (NIW) crossing the sound field in a shallow water environment. The impetus for this research stems from acoustic measurements taken during the Shallow Water '06 (SW06) field experiment, where a NIW traversed the water column such that soliton wavecrests were nearly parallel to the source-receiver path. Horizontal refraction effects are important in this scenario. A brief derivation of the parabolic equation (PE) that includes azimuthal coupling, which is often assumed negligible, is provided. Considerations relevant to this modeling effort are described, including: source parameters, bottom assumptions, the choice of reference sound speed, and the approach to modal decomposition of the PE field. A sound speed profile adapted from experimental SW06 data is used to simulate the NIW, assuming variations along the wavecrests (e.g. curvature) are negligible. Broadband and modal energy metrics show acoustic fluctuations due to internal wave activity. Repeated model runs simulate the NIW crossing the PE field over space and time. Statistical analysis shows the PE data is best fit by a lognormal distribution, but tends to an exponential distribution during certain scenarios. Small angle differences between the acoustic track and the propagating NIW cause substantial differences in energy distribution throughout the PE field. Refraction prior to the NIW's arrival is important, but can be considered a second-order factor in the perfectly parallel condition, where focusing effects will dominate.

(C) 2011 Acoustical Society of America

PACS numbers: 43.30.Es, 43.30.Re, 43.30.Dr, 43.30.Zk 


\subsection{INTRODUCTION AND BACKGROUND}

Since its introduction to the underwater acoustics community in $1977,{ }^{1}$ the parabolic equation (PE) model has been applied to a wide variety of complex oceanographic environments. One particular problem that has drawn an abundance of attention in recent years is the existence of nonlinear internal waves (NIWs), and their impact upon shallow water acoustics. ${ }^{2}$ Large scale ocean acoustic experiments, including PRIMER, ${ }^{3}$ SWARM, ${ }^{4}$ ASIAEX,,${ }^{5,6}$ and more recently the Shallow Water '06 (SW06) experiment, ${ }^{7}$ have significantly improved our understanding of these phenomena, and their relative importance to the sound field. Throughout these endeavors, modeling has played a vital role in interpreting experimental results, and advancing our knowledge base in this field of study.

An experiment in the Yellow Sea is often referred to as the starting point for investigations in this area, where it was found that internal waves were responsible for "anomalous" acoustic fluctuations, that have since become trademark features of NIW activity. By using PE simulations and normal mode decomposition, mode-coupling was the suggested dominant physical mechanism responsible for changes in measured signals propagating through NIW packets. ${ }^{8}$ Following this, a theoretical study implementing the parabolic approximation in the horizontal plane with vertical modes along the depth dimension treated horizontal refraction due to NIW activity, introducing focusing and defocusing effects when the acoustic path is parallel to soliton fronts. ${ }^{9}$ Shortly thereafter, fully 3-D PE techniques were used to simulate the time evolving NIW field, ascertain azimuthal dependence, and examine three-dimensional propagation effects. ${ }^{8-17}$ More recently, PE models have been used to analyze increasingly complex scenarios that are 
especially relevant to circumstances when the source-receiver path is nearly parallel to the NIW wavecrests, and where horizontal refraction dominates. ${ }^{18-20}$ This subject is also the primary goal of this work.

During the SW06 experiment, the Research Vessel Sharp transmitted acoustic signals in an area where NIWs often occur, and at various bearings and ranges relative to a moored receiving array. The primary purpose of this exercise was to further explore the frequency and azimuthal dependence defined by Badiey et al., ${ }^{21}$ and to collect data which verified acoustic fluctuations due to NIW activities. Since then, several NIW events experienced during the vessel's three-week deployment have shown evidence of horizontal refraction effects, including focusing, defocusing, and Lloyd's mirror phenomena. ${ }^{18,22}$ One event in particular, labeled "Event 44", offered a beautiful representation of NIW activity characteristic of SW06 data. While the initial intent of this study was to better understand Event 44 as a stand-alone example, it has been expanded, and considers a more general argument where the acoustic track is nearparallel to a very long and non-curved NIW train. By simulating a three-dimensional representation of a progressing NIW over both time and space, we create a statistical sample set that can be exploited. Specifically, we explore changes caused by very small angle differences, and time varying fluctuating regimes.

Our paper is organized in the following manner; we discuss some important background theory and considerations applicable to our PE model. Following this we present some specific examples of NIW activity that illustrate refraction, defocusing, and focusing effects. We simulate the NIW propagating over time and space by executing repeated model runs, each with a unique sound speed profile that advances the soliton 
train in small increments. We show the differences between virtual receivers, each with a slightly different angle deviation in the acoustic track. A statistical analysis of modeled data serves as a reference point across example scenarios.

\subsection{MMPE THREE-DIMENSIONAL PARABOLIC EQUATION MODEL}

The primary tool used in this analysis was the Monterey-Miami Parabolic Equation (MMPE) model, an outgrowth of the University of Miami Parabolic Equation Model

(UMPE), ${ }^{23}$ chosen for its ability to predict horizontal refraction effects in a threedimensional shallow-water environment. We provide a succinct derivation of the most important aspects of the algorithm based primarily upon $S_{m i t h}{ }^{24}$ and Smith and Tappert. ${ }^{23}$

The three-dimensional MMPE model is derived by representing a time-harmonic sound field $P$ in the Cartesian coordinate system with radial frequency $\omega$ as

$$
P(x, y, z, \omega t)=p(x, y, z) e^{-j \omega t} .
$$

Substituted into the three-dimensional Helmholtz equation gives

$$
\frac{\partial^{2} p}{\partial x^{2}}=\frac{\partial^{2} p}{\partial y^{2}}+\frac{\partial^{2} p}{\partial z^{2}}+k_{0}^{2} n^{2} p=-4 \pi P_{0} \delta\left(\vec{x}-\vec{x}_{s}\right)
$$

Here, the reference wavenumber $k_{0}$ is dependent on the reference sound speed $c_{0}$ by $k_{0}=\omega / c_{0}$. The acoustic index of refraction $n$, is determined by

$$
n(x, y, z)=\frac{c_{0}}{c(x, y, z)},
$$


with $c(x, y, z)$ being the three-dimensional sound speed profile, and also the primary environmental water-column variable that induces perturbations into the acoustic field. Density variations are treated at the water-bottom interface, as described by Smith. ${ }^{23}$ The right hand side of (3.2) takes into account an acoustic source with pressure amplitude $P_{0}$ defined at a one meter reference distance $R_{0}$. The source is positioned at zero range and arbitrary depth (such that $x=x_{s}=0, y=y_{s}=0, z=z_{s}$ ), and the point source contribution is given by the Dirac delta function

$$
\delta(\vec{x})=\frac{1}{2 \pi r} \delta(x) \delta(y) \delta\left(z-z_{s}\right) .
$$

To further simplify the Helmholtz equation, we introduce the differential operator notation

$$
\begin{aligned}
& P_{o p}=\frac{\partial}{\partial x} \\
& Q_{o p}=\sqrt{\mu+\varepsilon+v+1}
\end{aligned}
$$

where

$$
\begin{aligned}
& \varepsilon=n^{2}-1 \\
& \mu=\frac{1}{k_{0}^{2}} \frac{\partial^{2}}{\partial z^{2}} \\
& \nu=\frac{1}{k_{0}^{2}} \frac{\partial^{2}}{\partial y^{2}},
\end{aligned}
$$

so that we can write the homogeneous form of the Helmholtz equation as

$$
\left[P_{o p}^{2}+k_{o p}^{2} Q_{o p}^{2}\right] p=0
$$


Equation (3.7) can be factored into a form that represents incoming and outgoing waves. However, we will assume that backscattered energy is negligible, and limit ourselves to an outward propagating field, satisfied by

$$
\begin{aligned}
& p=Q_{o p}^{-1 / 2} \Psi \\
& P_{o p} \Psi=j k_{0} Q_{o p} \Psi
\end{aligned}
$$

where $\Psi$ is the PE field function, comprised of a slowly modulating envelope function $\psi$, and phase term dependent on the acoustic frequency. Defining the $x$ direction as the forward propagating field, we restrict (3.8) to

$$
\Psi=\psi e^{j k_{0} x}
$$

and,

$$
p=P_{0} Q_{o p}^{-1 / 2} \psi e^{j k_{0} x}
$$

Then the outgoing PE field in Cartesian coordinates can be defined as

$$
\frac{\partial \Psi}{\partial x}=e^{j k_{0} x}\left(\frac{\partial \psi}{\partial x}+j k_{0} \psi\right) .
$$

Substituting a normalized version of equation (3.10), where $|\psi|=1$ and $|p|=P_{0}$, into the Helmholtz equation yields

$$
\frac{\partial \psi}{\partial x}=j k_{0}\left(Q_{o p}-1\right) \psi
$$

If we introduce a Hamiltonian-like operator $H_{o p}$, which describes $Q_{o p}$ by its potential and kinetic energy components, and also defines the evolving PE field with range, 


$$
H_{o p}=1-Q_{o p}
$$

we can rewrite (3.12) as

$$
\frac{\partial \psi}{\partial x}=-j k_{0} H_{o p} \psi
$$

Now, because we have cast the above problem in a form where the PE field is a defined in the $(y, z)$ plane, we can represent the field as a function of increasing range with the use of a propagator function $\Phi(x)$

$$
\psi(x+\Delta x)=\Phi(x) \psi(x),
$$

This becomes the starting point for the numerical implementation of the parabolic acoustic wave equation, suitable for a split-step Fourier (SSF) approach. The advantage of the SSF method is that each individual operator within $Q_{o p}$ or $H_{o p}$ can be multiplied in either the spatial ( $y, z$-space) or Fourier transform $\left(k_{y}, k_{z}\right.$-space) domain - the domain choice made to lessen computational burden. However, these operators in (3.5) and (3.6) are a combination of scalar and differential forms. Therefore, the operator terms must be separated, which requires an approximation to their joined dependence by the square-root (see equation (3.5)).

The often-used wide-angle approximation ${ }^{25}$ can then be employed by letting

$$
Q_{o p} \approx 1-T_{2 D W A P E}+U_{\text {WAPE }},
$$

where 


$$
\begin{aligned}
& T_{2 D W A P E}=1-\sqrt{1+\mu+v=1}-\sqrt{1+\frac{1}{k_{0}^{2}} \frac{\partial^{2}}{\partial y^{2}}+\frac{1}{k_{0}^{2}} \frac{\partial^{2}}{\partial z^{2}}} . \\
& U_{W A P E}=1-\sqrt{1+\varepsilon=}-[n-1]
\end{aligned}
$$

It is important to note the non-separability between the $T_{2 D W A P E}(y$ and $z$ ) differential operators. Neglecting the $y$-dependence would return the $T_{2 D W A P E}$ term to the twodimensional single depth-slice approximation. However, retaining this term allows for cross-range variation, or "horizontal coupling" along the $y$ dimension. Retaining this $y$ -dependence is similar to retaining azimuthal dependence in the cylindrical derivation, rather than assuming an uncoupled azimuthal approximation (UNCA). ${ }^{24}$ For our problem at hand, this dependence is tremendously important, so that we adequately capture refractive effects due to NIW activity. Additionally, including the $y$ differential operator helps account for cylindrical spreading, and consequently must be included in the threedimensional Cartesian derivation.

Equation (3.15) can be represented, and implemented, as a range-step marching algorithm,

$$
\begin{aligned}
& \psi(x+\Delta x, y, z) \approx e^{-j k_{0} \frac{\Delta x}{2} U_{\text {WAPE }}(x+\Delta x)} \times \ldots \\
& \quad \operatorname{IFFT}^{2 D}\left\{e^{j k_{0} \Delta x\left[\hat{T}_{\text {WAPE }}\left(k_{z}\right)+\hat{V}_{\text {WAPE }}\left(k_{y}\right)\right]} \times F F T^{2 D}\left[e^{j k_{0} \frac{\Delta x}{2} U_{\text {WAPE }}(x)} \psi(x, y, z)\right]\right\}
\end{aligned}
$$

with,

$$
\left[\hat{T}_{2 D W A P E}\left(k_{z}\right)+\hat{V}_{2 D W A P E}\left(k_{y}\right)\right]=1-\sqrt{1-\left(\frac{k_{z}^{2}+k_{y}^{2}}{k_{0}^{2}}\right)} .
$$


Here, $U_{o p}$ is numerically simple in the spatial domain, as shown by (3.17). However both $V_{o p}$ and $T_{o p}$ are differential operators. Transforming them into the Fourier domain converts them to the scalar operators given by (3.19). This algorithm is known as a centered-step scheme, and provides the general mathematical basis for the MMPE algorithm used in this study. Several other factors are important to consider, and those pertinent to this work are briefly touched upon in the following sections.

\section{A PE source and bandwidth}

The version of MMPE code utilized in this study is implemented such that range is represented along the $x$-axis, cross-range along the $y$-axis, and depth along the $z$-axis. Because we are interested in three-dimensional refraction effects, we desire the maximum amount of energy possible to be distributed in the lateral (cross-range) dimension.

MMPE implements a wide angle source function based on the Thomson and Bohun field starter. $^{26}$ In order to avoid sidelobes, a taper is introduced in the $k_{z}, k_{y}$ domain. Modifications were made to the original code in order to widen the azimuthal aperture in the cross-range $\left(k_{y}\right)$ domain, yielding a maximum source angle of up to $85^{\circ}$. The source-angle taper was not altered in the depth $\left(k_{z}\right)$ domain, and assumed valid for angles up to $40^{\circ}$.

During the experiment, the J-15 source was located at 74 meters depth and transmitted linear frequency modulated chirp signals between 50 and $450 \mathrm{~Hz}$. During the acoustic analysis of measured experimental data, time-integrated intensity $I_{\tau}$ (a measure of energy) served as our primary metric to evaluate fluctuations induced by NIW activity 


$$
I_{\tau}(z, k)=\int I(\tau, z, k) d \tau
$$

In equation (3.20) the integration takes place at each phone depth $z$, on each chirp arrival $k$, and over the energetic region of the received signal $\tau$. These data were highpass filtered at a $100 \mathrm{~Hz}$ cutoff frequency to limit low frequency ocean noise. To directly compare modeled versus measured data, one would need to sufficiently model the signal in frequency, and approximate the time arrival via an inverse Fourier transform. However, even with the computational resources at hand, we could not sufficiently sample the chirp bandwidth and also simulate the progression of the NIW over space. Therefore we offer an approximation of received energy by applying Parseval's theorem. Let our received signal be represented in the time domain by $P(t)$ and in the frequency domain by $p(f)$,

$$
\int_{\tau}|P(t)|^{2} d t=\int_{B W}|p(f)|^{2} d f
$$

Because energy is conserved in both the time and frequency domains, instead of an integral over time $\left(I_{\tau}\right)$, we perform the calculation over frequency and denote it by $I_{f}$. Therefore, the approximate energy of the acoustic signal based on several frequency samples can be written as

$$
I_{f}=\int_{B W}|p(f)|^{2} d f \approx \frac{B W}{N} \sum_{i=1}^{N}\left|p\left(f_{i}\right)\right|^{2}
$$

where $B W$ represents the bandwidth of the signal, modeled at $N$ discrete samples. For this study we limited the modeled signal bandwidth to $100-300 \mathrm{~Hz}$, evaluated at $N=17$ 
(12.5 Hz sampling). This resolution was the upper limit of our computational resources. Although this is a relatively coarse representation of the signal bandwidth, the overall energy fluctuations are well-captured. APPENDIX B discusses the impacts of this tradeoff in further detail.

The source frequency also plays an important role in determining the spatial grid of the PE model. In the "accuracy" mode, MMPE samples the water column at one-tenth of one wavelength in depth (to accurately treat bottom density contrasts), one-half of one wavelength in cross-range, and at one wavelength for each range step. Because our calculation of $I_{f}$ requires a summation at a fixed point in space, and across $N$ frequencies, we require the spatial grid to be fixed across our source bandwidth. Choosing an appropriate spatial sampling grid became an important factor when choosing the modeled signal's bandwidth. Incorporating frequencies above $300 \mathrm{~Hz}$ would require over-sampling the PE field at $100 \mathrm{~Hz}$. Similarly, considering frequencies below $100 \mathrm{~Hz}$ would potentially under-sample the PE field above $300 \mathrm{~Hz}$. Consequently, a slightly narrower bandwidth than the $50-450 \mathrm{~Hz}$ SW06 chirp signal was considered. A spatial grid corresponding to $175 \mathrm{~Hz}$ allowed for acceptable sampling over a $100-300 \mathrm{~Hz}$ bandwidth, and was chosen as the final configuration for our investigation.

\section{B Bottom considerations}

Because we are primarily concerned with water-column induced acoustic perturbations, we only consider a flat bottom with a constant depth of 80 meters. This simplification is relatively true of the SW06 experimental area, which varied no more than \pm 2.5 meters over the Event 44 acoustic track. However, because sea bottom properties are expected to have a relatively profound impact upon the amount of acoustic 
energy that remains trapped in the water column, a sensitivity analysis of bottom parameters was performed.

The MMPE algorithm incorporates a two-layered bottom approximation where, within each layer $n$, one can account for the medium density $\rho_{n}$, compressional speed $c_{p n}$, compressional attenuation $\alpha_{p n}$, shear speed $c_{s n}$, and shear attenuation $\alpha_{s n}$. Because the abrupt transition between water-column and bottom layer properties can create mathematical artifacts when implementing a Fourier transform, the MMPE algorithm creates a smoothed profile at this sharp interface. The layers are modeled by an equivalent fluid approximation given by Tindle and Zhang, ${ }^{27}$ where the layered solid is replaced by a fluid with adjusted parameters. The approach is suitable for low shear speeds, and approximates the bottom reflection coefficient well. Attenuation is accounted for by an imaginary term within the density, which is important to account for when decomposing the PE field into normal modes.

Previous estimates from geoacoustic inversions by Jiang, et al. ${ }^{28}$ and Potty, et al. ${ }^{29}$ provided bounds for the eleven possible input parameters, shown in Table 3.1and Table 3.2. By varying these eleven parameters between realistic maximum and minimum values, 2048 (equivalent to $2^{11}$ ) individual two-dimensional MMPE realizations were accomplished. A simulated $300 \mathrm{~Hz}$ acoustic source located at 74 meters depth imitated the experimental configuration; this frequency chosen because it was the upper limit of our modeled bandwidth, and because higher frequencies are expected to be more sensitive to the varying parameters. Depth integrated intensity $I_{z}$, was calculated for each individual model run in order to explore the influence these parameters had upon 
water column energy. Figure 3.1 shows $I_{z}$ curves between five and ten kilometers from the modeled source. The four panels show identical curves for all 2048 model executions, but are separated into colorized (blue or red) "bands" that delineate the top four parameters influencing acoustic energy being transferred into the bottom. The green curve represents the depth integrated intensity for the "optimal" case, which corresponds to the most likely (mean) values from the inversion results. The top left panel shows layer-one compressional attenuation to be the most influential factor, separating the model runs above and below the optimal scenario. The second order banding is due to layer-one compressional speed dependence, followed by layer-one shear speed dependence. Density in the top layer creates the smallest banding effect. Because these parameters stem from inversion results, it is prudent to note that parameter estimates that created the first and second order bands (compressional attenuation and compressional speed) were well estimated. However, the third and fourth parameters (shear speed and density) were not as well estimated and carried wider limits. Therefore, the "banding" taking place for these last two parameters may be a consequence of the wide range between their expected minimum and maximum values. One cannot ignore the importance of other parameters (such as layer height) that have not been specifically singled out by Figure 3.1. Because the mean (or optimal) values are centered across the range of possibilities, they were chosen as the final input parameters to the MMPE algorithm. 
Table 3.1 Layer 1 geoacoustic parameters

\begin{tabular}{lccc}
\hline \hline & Minimum & Mean & Maximum \\
Density, $\rho_{1}\left(\mathrm{~kg} / \mathrm{m}^{3}\right)$ & 1.63 & 2.09 & 2.53 \\
Compressional speed, $c_{p 1}(\mathrm{~m} / \mathrm{s})$ & 1653.4 & 1683.75 & 1709.5 \\
Compressional attenuation, $\alpha_{p 1}(\mathrm{~dB} / \mathrm{m} / \mathrm{kHz})$ & 0.057 & 0.172 & 0.274 \\
Shear speed, $c_{s 1}(\mathrm{~m} / \mathrm{s})$ & 0 & 161.45 & 362 \\
Shear attenuation, $\alpha_{p 1}(\mathrm{~dB} / \mathrm{m} / \mathrm{kHz})$ & 0.24 & 0.754 & 1.248 \\
Layer height, $H_{1}(\mathrm{~m})$ & 15 & 22.65 & 31.8 \\
\hline \hline
\end{tabular}

Table 3.2 Layer 2 geoacoustic parameters

\begin{tabular}{lccc}
\hline \hline & $\underline{\text { Minimum }}$ & $\underline{\text { Mean }}$ & Maximum \\
Density, $\rho_{2}\left(\mathrm{~kg} / \mathrm{m}^{3}\right)$ & 1.901 & 2.361 & 2.8 \\
Compressional speed, $c_{p 2}(\mathrm{~m} / \mathrm{s})$ & 1982 & 2164.5 & 2354 \\
Compressional attenuation, $\alpha_{p 2}(\mathrm{~dB} / \mathrm{m} / \mathrm{kHz})$ & 0.117 & 0.378 & 0.633 \\
Shear speed, $c_{s 2}(\mathrm{~m} / \mathrm{s})$ & 139.9 & 825.2 & 1552.6 \\
Shear attenuation, $\alpha_{p 2}(\mathrm{~dB} / \mathrm{m} / \mathrm{kHz})$ & 0.089 & 0.857 & 1.635 \\
\hline \hline
\end{tabular}




\section{Modal analysis and reference sound speed sensitivity}

To better understand acoustic propagation in complex environments, it is often useful to decompose the field into its normal modes. By doing so, one can understand if modes are propagating adiabatically, or if environmental disturbances are inducing mode coupling effects. Because the MMPE algorithm provides the full acoustic field, modal information can be obtained in a post-processing manner by decomposing the PE field into its normal mode components. In the case of the standard parabolic equation (SPE), ${ }^{1}$ the field can be related to the normal modes of the Helmholtz equation in an exact manner. The wide angle approximation (WAPE) ${ }^{30}$ given by equation (3.17) is generally chosen instead in split-step Fourier implementations, because it reduces phase errors associated with the SPE, ${ }^{31}$ expands the useful angle of propagation, and has been found to be less sensitive to the choice of reference wavenumber $k_{0} \cdot{ }^{32}$ However, the form of the WAPE approximation to the Helmholtz equation does not maintain the proper form of the depth separated operator. Therefore, the standard normal mode basis set is not formally a proper eigenfunction basis set of this operator. Smith and Smith ${ }^{33}$ showed that eigenfunctions corresponding to the WAPE basis set are dependent on the choice of $k_{0}$ (or $c_{0}$ ). It was also shown that the WAPE eigenfunctions are close, but not exactly identical to those of the SPE, or the Helmholtz equation. Therefore, proper choice of $c_{0}$ may result in a WAPE basis set more closely aligned with normal modes associated with the Helmholtz equation. This would infer that if $c_{0}$ has been properly optimized, decomposition of the PE field using standard normal modes would yield improved phase matching, and "smoother" results. 
The appropriate choice of $c_{0}$ was the premise of the $c_{0}$-insensitive parabolic equation (COIPE) formulation suggested by Tappert. ${ }^{34}$ In this case, the reference sound speed is calculated as a function of range $r$ by

$$
c_{0 I P E}(r)=\left[\frac{1}{h(r)} \int_{0}^{h(r)} \frac{1}{\sqrt{c(z, r)}} d z\right]^{-2},
$$

where $h(r)$ is the water column depth and $c(z, r)$ is the sound speed profile. In this proposed schema, although the reference sound speed is allowed to vary with range, reciprocity still holds in a range-dependent environment. The variation in $c_{0 I P E}$ due to NIW perturbations is shown in Figure 3.2.

It is important to note that the COIPE method does much more than refine the calculation of the reference sound speed. The approach has been shown to increase the accuracy of the WAPE approximation to second order in the depth-separated operator. ${ }^{32}$ This increases the phase accuracy of the solution, decreases further the sensitivity to the reference sound speed, and increases the match between the formal eigenvectors of the approximation and the standard normal modes of the Helmholtz equation. However, the computational revisions necessary to adapt the model were considered too extensive for this work, and would not be expected to produce significantly different results, if proper care is taken to address the issues with the existing code.

Because we are interested in modal dependence of the sound field in the presence of internal waves, the choice of $c_{0}$ may impact the success of our modal decomposition. Therefore, a sensitivity analysis was carried out, varying $c_{0}$ over a variety of ranges and 
the relative "flatness" of our modal decomposition was examined. A single sound speed profile $c(z, r)$ was calculated by averaging measurements captured on environmental mooring SW32 for two hours prior to Event 44's arrival. Iterative two-dimensional MMPE runs varied sound speed across the minimum and maximum of this profile at $\Delta c_{0}=0.5 \mathrm{~m} / \mathrm{s}$ increments for 100,200 and $300 \mathrm{~Hz}$ source frequencies. Additionally, the $c_{0 I P E}$ value calculated by equation (3.23) was also used as a comparison point since it is expected to perform well. For each model realization, the PE field was decomposed into the first eight normal modes using the KRAKEN normal mode program. ${ }^{35}$ After accounting for cylindrical spreading, the modal amplitudes were compared against expected modal attenuation, using the modal attenuation coefficient $\delta_{m, e}, 36,37$

$$
\delta_{m, e}=\frac{\omega}{k_{m}} \int_{0}^{\infty} \frac{\alpha_{e}(z)}{c(z)} \Psi_{m}^{2} d z
$$

where $\omega$ is the radial frequency, $k_{m}$ the horizontal modal wavenumber, $\alpha_{e}(z)$ the depthdependent exponential attenuation factor, and $\Psi_{m}(z)$ the mode function. In addition, modal amplitudes were also compared to a least-squares exponential fit. Figure 3.3 shows an example $c_{0}$-iteration where modal decomposition of the PE field is compared to expected attenuation profiles. The residuals between actual and expected $\left(r_{i}\right)$, are plotted for each mode. The norm of the residuals $r_{n}$, is annotated as a metric for "goodness of fit"

$$
r_{n}=\sqrt{\sum_{i=1}^{n} r_{i}^{2}}
$$


One hundred $c_{0}$ iterations were accomplished at each source frequency. To compare the "flatness" of each mode, $r_{n}$ was compared across frequencies to see if an optimal reference sound speed was possible. The entire $c_{0}$ spread was also directly compared to $1500 \mathrm{~m} / \mathrm{s}, c_{0 I P E}$, and the mean water column sound speed. In the end, there are certain values which can optimize the smoothness of modal decomposition. However, the values were not found to be consistent across mode number nor frequency. Further, the "flatness" differences between the entire $c_{0}$ spread was found to be relatively small. Therefore, a standard reference sound speed of $1500 \mathrm{~m} / \mathrm{s}$ was chosen, as it is a wellaccepted value.

\section{D "Event 44" internal wave sound speed profile}

The primary motivation for this study was to better understand one specific experimental dataset from the SW06 experiment. On 14 August 2006 the R/V Sharp witnessed a substantial NIW event on marine radar while it transmitted broadband (50$450 \mathrm{~Hz}$ ) chirp signals from a J-15 acoustic source, moored at 74 meters depth. These signals were recorded by a 16-element vertical line array, $15 \mathrm{~km}$ away from the source. The experimental geometry was such that the source-receiver path happened to be nearparallel $\left(\approx 5-6^{\circ}\right)$ to the NIW wavefronts. This NIW event, labeled "Event 44 " was captured on several oceanographic sensors, including environmental mooring SW32; which served as the primary instrument to reconstruct the phenomenon as it propagated over the acoustic track. Event 44 propagated at roughly $0.5 \mathrm{~m} / \mathrm{s}$, considerably slower than expected, since these waves tend to travel between 0.7 and $1.0 \mathrm{~m} / \mathrm{s}$. Based upon measurements captured by the environmental sensors, we can safely assume the NIW 
covered the entire $15 \mathrm{~km}$ acoustic track. We can also assume that there was little (or no) curvature to the wavefront, and that the first soliton arrived at the receiving VLA roughly one hour before reaching the J-15 source. Experimental acoustic data recorded during Event 44 shows escalating energy prior to the wavefront arriving at the source and receiver. Once the NIW train crossed into the acoustic track, the energy received on the array fluctuated substantially. The reader is referred to Chapters 1 and 2 for more details regarding the exact oceanography and measured acoustic data that transpired during this time period.

The primary challenge in modeling Event 44 was that we did not have sufficient sampling of the water column to adequately capture a detailed wave structure. We have reasonable knowledge of NIW activity at the source, the receiver, and a midway point at environmental mooring SW32. These time records provide brief snapshots of activity, but are not sufficient to confidently model the wave's evolution in both space and time. Therefore, three noteworthy assumptions were made:

1. We assume that the time series captured by the environmental sensors represent a valid approximation of the NIW's structure in space. This assumption infers that the wave evolution is frozen as it traverses the acoustic track; and that it does not significantly undulate over this period of time.

2. Based upon our interpretation of the marine radar, we assume that the NIW can be modeled in a straight-line fashion. In other words, the solitons (and especially the leading edge) contain no curvature. NIW curvature has been shown to be acoustically important when it exists. ${ }^{20}$ 
3. For simplicity, we assume that the NIW does not vary in range. Therefore, the internal wave activity captured at environmental mooring 32 (situated midway between the source and receiver) adequately represents the NIW's structure over the entire $15 \mathrm{~km}$ acoustic track.

Our third assumption was not made lightly. NIW activity at the source and midway point show strong similarity over this portion of the acoustic track. However, NIW activity detected at the receiving array significantly differs. Instead, environmental sensors captured weaker NIW development. Perhaps this is because the NIW arrived at the receiving array one hour prior to the source, was less mature, and therefore contained fewer solitons. Another possibility is that the solitons tapered with range, and the receiver was located at the far edge of the NIW train. A previous study treating truncated NIWs showed this is an important consideration. ${ }^{19}$ To justify our third assumption, a full three-dimensional sound speed profile based upon all three measurement locations was used in several trial MMPE iterations. While the results from this range-dependent scenario could mimic the reality of Event 44 better, we implemented a "walk before you run" approach. The more simple (range independent) scenario is easier to interpret, and can provide more conclusive results that are applicable to general NIW phenomena versus a single event.

The Cartesian version of MMPE requires the sound speed profile to be in the form of a rectangular ocean volume. Because we decided to treat the problem as a rangeindependent scenario, we required only a single "slice" of the ocean to be imported into the algorithm when initialized, to be reused for each forward marching increment. To create this sound speed profile, we implemented a 2-D linear interpolation of measured 
data captured by environmental mooring SW32. Sound speed was derived ${ }^{38}$ from six $^{2}$ pressure, salinity, and temperature sensors, spanning the water column between 12 and 75 meters, each sensor sampling every 30 seconds. Because the uppermost sensor for SW32 was lost at sea, sound speed data above 12 meters was derived by averaging seasurface sensors from adjacent environmental moorings. We converted all time-series measurements into a spatial representation by assuming a constant NIW speed of $0.5 \mathrm{~m} / \mathrm{s}$. In addition to NIW activity, environmental mooring SW32 also captured a thermohaline bottom intrusion, often referred to as the "foot of the front," which creates a mid-water

column sound speed minimum. Based upon other SW06 sensors and recent literature, ${ }^{39}$ the bottom intrusion can be approximated as a uniform layer across the entire modeled area. Therefore, the sound speed profile between the lowest sensor and the ocean floor (between 75 and 80 meters) was considered to be constant. Both the NIW and bottom intrusion were incorporated into an input file suitable for import into MMPE, where special care was taken to use identical grid spacing, and to insert the water-column profile immediately above the smoothed water- bottom discontinuity. The Event 44 sound speed profile is offered as a reference in the upper panel of Figure 3.2

\subsection{IMPACTS OF NIW ACTIVITY UPON THE SOUND FIELD}

Figure 3.4 depicts the general acoustic problem that we consider in this study, which was inspired by Event 44 from the SW06 experiment. A relatively broadband (100-300 $\mathrm{Hz}$ ) moored acoustic point source is located at 74 meters depth, $15 \mathrm{~km}$ away from a vertical line receiving array. A slow moving NIW train propagates over time and space such that it is nearly parallel the source-receiver path. It is assumed that the NIW does not vary with range and that it does not possess any curvature along the soliton 
wavecrests. The exact angle between the acoustic track and the propagating NIW, $\alpha$, is not known but believed to be between \pm 5 degrees. The small angle dependence is illustrated by a NIW shown at two instances in time (or position), with arrays situated at possible locations relative to the wavecrests.

In the following sections we describe three discrete horizontal refraction scenarios: refraction (prior to the NIW's arrival), defocusing (arrival at the acoustic source), and focusing (trapping of the acoustic source between solitons). These are provided to show the dominant physical mechanisms that cause fluctuations in the sound field. Secondly we simulate the NIW propagating over the modeled area, and show how combinatory horizontal refraction effects create fluctuations that have become trademark signatures of NIW activity.

\section{A Discrete horizontal refraction scenarios}

Figure 3.5 highlights the three dominant horizontal refraction effects that cause fluctuations in the sound field. The top-left panel shows the sound speed profile from Event 44 that was introduced into the MMPE algorithm. The acoustic source is marked by a star. Although the NIW train does not directly perturb sound speed at the acoustic source, it is close enough that a refraction scenario will play out. The profile is valid across range ( $y$-axis), and the impact of the NIW's leading edge becomes apparent when examining the integrated energy plot shown directly below. Energy spread from the 100 $\mathrm{Hz}$ source reaches the first soliton, and much of it is refracted, forming an interference pattern that can be interpreted as a horizontal Lloyd's mirror. ${ }^{18,40}$ The interference pattern creates "fingers" of energy in the horizontal plane which dominate the left side of modeled area, and correspond to fluctuations (at $100 \mathrm{~Hz}$ and $15 \mathrm{~km}$ ) more than $2 \mathrm{~km}$ in 
advance of the NIW. These fluctuations are further clarified by the bottom-left panel which shows transmission loss (TL) at $15 \mathrm{~km}$ range versus depth, demonstrating the Lloyd's mirror interference pattern also varies with depth. The center panels represent a defocusing situation, as the leading soliton centers itself over the acoustic source. Here energy diverges laterally, as evidenced in both the depth integrated energy and TL plots. In this example, one would expect a deep signal fade at the receiver. In contrast, the right-most panels represent a focusing event, as two solitons trap the acoustic source and form a sound channel in the horizontal plane. A substantially amplified signal would be expected if the receiver was located within the transient waveguide.

These three examples highlight how over very short timescales (minutes) NIW activity creates significant modulations in the acoustic field. Additionally, there are some higherorder intricacies that can be inferred from the depth integrated intensity plots. In all three instances, the modeled areas containing the NIW disturbances show complex interference patterns. In these portions of the modeled area, secondary ducting and refraction of energy appears between succeeding soliton channels. To better understand the subtleties associated with NIW modulations, a modal analysis is helpful.

\section{B Modal dependence}

Our approach to modal analysis was to decompose the full PE field as a postprocessing task, rather than initialize the PE field with a modal starter. The expected eigenfunctions for the acoustic field were calculated by the KRAKEN normal mode program $^{35}$ using identical MMPE sound-speed profiles and bottom parameters. The PE field was then decomposed into normal modes by applying the sample mode shape approach. ${ }^{41}$ As indicated earlier, the WAPE field does not exactly break down into a 
normal mode basis set. However, by exactly matching parameters between KRAKEN and MMPE, we minimize the phase errors that can often occur in this approach. Anomalies from the normal mode decomposition of the WAPE field begin to appear as a "banding" effect in some of the highest modes (i.e. Figure 3.14). Figure 3.6 shows the influence of NIW activity on modeshapes for the first five modes at $300 \mathrm{~Hz}$. The upperleft panel shows the sound speed profile based on true experimental data. Each soliton perturbs the modeshapes such that the relative maxima and minima are pushed deeper into the water column. It is important to note that this profile also includes the "foot of the front" which can help protect higher order modes from attenuating into the bottom.

Following our $100 \mathrm{~Hz}$ example in Figure 3.5, we show mode contributions for the same scenarios at $300 \mathrm{~Hz}$. This frequency chosen as it was the upper limit of our modeled bandwidth, offers a balance to the $100 \mathrm{~Hz}$ examples, and also because modal refraction is generally more evident at higher frequencies. In these cases, modal refraction is determined by Snell's law, written in terms of the modal eigenvalues,

$$
k_{r m} \cos \theta=k_{r m}^{\prime} \cos \theta^{\prime}
$$

where the prime indicates a transition to a second (perturbed) state. The critical angle for complete refraction is therefore

$$
\theta_{c}=\cos ^{-1}\left(\frac{k_{r m}^{\prime}}{k_{r m}}\right) \text {. }
$$

Figure 3.7 and Figure 3.8 show the first ten modes in a refraction scenario, referenced to the input sound speed profile and $300 \mathrm{~Hz}$ depth integrated intensity. Because of the very low source depth, mode 1 was not heavily excited. However, at cross-ranges past 
the initial soliton, mode coupling from the higher order modes transfers energy into mode 1. This transfer is especially remarkable at angles approaching an acoustic path perpendicular to the NIW front, which corroborates well with previously published results. ${ }^{21,40,42,43}$ Above mode 2, we see increased initial modal excitement, and looking across plots we notice that horizontal refraction effects generally increase as mode number increases. For example, mode 2 refraction into the unperturbed region (ahead of the NIW) is slight - while mode 5 refraction starts earlier and therefore extends across a wider cross-range aperture at $15 \mathrm{~km}$ range. Similarly, the shadow-zone created by the leading soliton also increases with higher modes. Figure 3.9 and Figure 3.10 illustrate a defocusing situation, with the general trend of widening shadow zones for increasing modes. Because energy is spread away from boresight (the perfectly parallel case), a substantial amount is vectored laterally. This indicates that a defocusing event may actually cause an increase in energy if the receiver is located sufficiently off-axis; this situation being quite likely for Event 44. Lastly, Figure 3.11 and Figure 3.12 depict focusing. We see that the horizontal sound channel has little energy from mode 1 , as it was not initially excited and is not easily trapped. However the higher order modes are well-trapped by the soliton waveguide. Additionally, modes 3 and above begin to show secondary ducting in succeeding soliton waveguides, suggesting the appearance of these effects in Figure 3.5 is an influence of higher order modes. APPENDIX C shows similar examples as Figure 3.7 - Figure 3.12, but with a varied source depth that better excites mode 1. 


\section{Frequency dependence}

Because we are using equation (3.22) to approximate the energy received from a broadband time arrival, based upon $N$ frequencies, it is prudent to examine the frequency dependence of NIW perturbations in the sound field. Figure 3.13 shows normalized intensity at $15 \mathrm{~km}$ range during a refraction scenario, indicating differences across the bandwidth are considerable. The Lloyd's mirror interference pattern in the cross-range (horizontal) dimension shows finer granularity at higher frequencies. Similarly, the depth (and likely modal) dependence is more detailed at $300 \mathrm{~Hz}$ versus 100 Hz. The broadband energy metric, $I_{f}$, that we use condenses this frequency variability into a single measure. In our analysis we have normalized this metric such that the mean is one; $\left\langle I_{f}\right\rangle=1$.

Figure 3.14 shows the frequency dependence for modes 1-5, compared to the broadband energy metric $I_{f}$, in a refraction scenario, and at $15 \mathrm{~km}$ range. Broadband energy is plotted immediately below the sound speed profile in two forms. When plotted versus depth, this can be interpreted as collapsing the plots shown in Figure 3.13 into one. The "point" measurements plot uses a similar approach to our treatment of measured data, and is well-suited for statistical analysis. In both plots, we see the effect of a horizontal refraction interference pattern as a series of peaks and nulls in energy versus

cross-range in the unperturbed portion of the water column. Also in Figure 3.14 are plots illustrating frequency dependence of each mode. A curtained effect is immediately recognizable, where the horizontal interference pattern shows strong frequency dependence for each mode. The important point to be made here is that the broadband 
interference pattern shown in $I_{f}$ is actually a combination of frequency-dependent and mode-dependent interference patterns.

Figure 3.15 represents the frequency dependence during a defocusing scenario. Interpreting the $I_{f}$ point observations, we see that the overall energy at $15 \mathrm{~km}$ has been very well spread from the direct parallel path. Energy in the unperturbed portion of the water column arguably has more energy than in the perturbed portion. Examining the frequency dependence, rather than the striped effect evident in the refraction condition, a blurring has occurred. This is because the leading soliton causes energy to immediately diverge away from the source rather than refract at a downrange point. Similar energy is vectored into the NIW train, but more complex frequency dependence is caused by the perturbed sound speed profile.

Figure 3.16 shows a focusing scenario, where the bulk of energy is well-trapped between the soliton-formed waveguide. In this instance the broadband metrics peak at almost forty times the average value of one; this is roughly a $16 \mathrm{~dB}$ increase (re $\left\langle I_{f}\right\rangle=1$ ). Examining the frequency dependence upon modal decomposition, we see braid-like patterns between the initial solitons. Because this waveguide is essentially bounded both horizontally and vertically, it can be considered to have modes in both dimensions, and can therefore be decomposed into modes in both regards. ${ }^{19}$ As frequency changes, different horizontal modes will be excited within the waveguide, creating the structure we see for each vertical mode.

We desire to collapse the frequency dependence within each mode to a singular value. Given our relatively low frequency sampling, we offer a measure of modal energy, 


$$
A_{m f}=\frac{B W}{N} \sum_{i=1}^{N}\left|A_{m}\left(f_{i}\right)\right|^{2},
$$

where $B W$ represents the bandwidth of the signal, modeled at $N$ discrete samples, for each mode $m$. Because we are squaring each mode individually, we cannot recreate $I_{f}$ from this basis set. However, it does provide a way to correlate the contribution from each mode $\left(A_{m f}\right)$ to the overall broadband energy metric $I_{f}$. In order to retain insight into the relative contribution for each mode, we normalize the entire modal basis set such that the mean energy is one for all calculated modes,

$$
\left\langle\left. A_{m f}\right|_{m=1 \rightarrow 10}\right\rangle=1
$$

We base our normalization on the first ten modes and over the 100-300 Hz bandwidth. This measure of modal energy is compared to broadband energy in Figure 3.17, Figure 3.18, and Figure 3.19. In all cases, mode 3 dominates energy delivery, and in most cases mode 1 is overshadowed by modes $2-7$, showing the importance of higher order modes when considering fluctuations due to NIW activity. To show the changes in modal energy $\Delta A_{m f}$, we normalize modes 1-10 according to unperturbed levels, so that in an unperturbed condition all modes are equal to one. For example, in the refraction scenario illustrated by Figure 3.17, $\Delta A_{m f}=1$ for all modes at $-3 \mathrm{~km}$ cross-range - since refraction effects do not dominate at this distance. However, closer to the approaching soliton (near zero cross-range), modal amplitudes change from their steady-state values. In the region influenced by the NIW train, mode coupling effects cause significant energy transfer to mode 1. The defocusing scenario in Figure 3.18 shows a tendency to transfer some 
energy into mode 1 in both the unperturbed region and the area affected by the NIW. Figure 3.19 shows a focusing condition, with a close-up of the horizontal sound channel $( \pm 0.5 \mathrm{~km})$ given by Figure 3.20. Modes 2-7 carry most energy throughout the threedimensional waveguide, but $\Delta A_{m f}$ shows significant energy is being trapped within (or perhaps coupled into) modes 1-10.

\section{Simulated time-dependent NIW propagation}

While these specific scenarios are useful in understanding the dominant physical processes at play, we lack a good understanding of the time-dependence associated with the NIW traversing the sound field. Several questions remain unanswered, many of which are important to the engineer, scientist, or naval operator who must deal with the shallow water environment. In this section we seek to better understand and quantify the overall expected energy fluctuations due to horizontal refraction effects, and the associated time-dependence. We also examine the significance of small angle changes between the source-receiver track and the propagating NIW

To simulate the propagating NIW field over space and time, we employed a bank of thirty computers over a one-week period. This corresponded to roughly 4,000 individual three-dimensional MMPE runs, which sampled the acoustic signal at seventeen frequencies over the 100-300 Hz bandwidth, and propagated the NIW ten kilometers in forty-meter marching increments. To put this in context, it is similar to modeling a water mass (with constant eighty-meter depth) the size of the Red Sea! Consolidating the frequency bandwidth into the energy metric $I_{f}$ reduces the problem to just over 200 marching steps, each with an energy profile at $15 \mathrm{~km}$ range. This yields a simulated time-varying "slice" of water column which we employ in a statistical analysis. 
Compiling all energy values for each water column slice provides a sample size of roughly fourteen million points. We have removed the absorbing boundary layers and the simulated ocean bottom in this sample set. This can also be interpreted as a very densely sampled rectangular array (sixty thousand elements) that is roughly six kilometers wide, and spans the entire eighty-meter water column. This array would be 15 km away from the acoustic source, and each element records 200 measurements as they witness the NIW train pass by. It is our goal to break these data into regimes that show spatial dependence (i.e., the impact on $\alpha$ ) and time dependence (i.e., NIW activity before versus during). Therefore, we require a reference value for both the mean and variance. We choose to use the mean and variance for the entire dataset, which we have normalized such that the overall mean is unity; $\left\langle I_{f}\right\rangle=1$.

\section{E Effects upon small angle variations}

Figure 3.21 shows a normalized histogram of the complete dataset, which is best fit by a lognormal distribution. One may argue the histogram is not particularly well represented by the lognormal PDF - however, in aggregate, these data are not appropriate for the Raleigh, exponential, nor Gaussian distributions. Further reason to utilize the lognormal distribution for our modeled data is because the measured data during Event 44 is well-fit by a lognormal PDF. Additionally, the lognormal distribution for modeled intensity fluctuations has been previously argued by Tang et al. ${ }^{44}$ where a numerical Monte Carlo study using UMPE simulations examined intensity scintillations due to the linear internal wave spectra. Choice of the lognormal PDF was further endorsed by Fredricks et al. ${ }^{45}$ where acoustic data from the PRIMER experiment was statistically analyzed for high-frequency oceanographic process modulations - specifically, at sub- 
tidal timescales (less than two hours) where the NIW field dominated. Finer sampling in our model data, perhaps either in frequency spacing or NIW marching increment, could possibly fill out this histogram to better match the lognormal (or alternate) PDF. However, the purpose of fitting our entire dataset is to create a reference point for breaking these data down further, and to choose the optimal approach for mean and variance calculations upon discrete portions of the data. Figure 3.21 annotates the lognormal mean and variance $(\hat{\mu}=1.06, \hat{\sigma}=1.36)$, and also the exponential mean and variance $(\hat{\mu}=1.02, \hat{\sigma}=1.04)$. While these data as a whole are best fit by the lognormal distribution, certain subsets tend to an exponential distribution, warranting this calculation as a separate reference.

The first dissection of these data is upon the relative angle between the acoustic track and the propagating NIW, $\alpha$. Recalling the original acoustic problem posed in Figure 3.4 , what will be the difference in received signals given different receiver locations? Suppose we have five receiving arrays at our disposal, and we position them according to Figure 3.4 at $\alpha=-4^{\circ},-2^{\circ}, 0^{\circ}, 2^{\circ}, 4^{\circ}$; where a negative bearing would correspond to the NIW arriving at the receiving array prior to the source. Figure 3.22 shows expected energy arrivals upon each array based upon repeated PE simulations. The five top-left panels show point measurements which simulate energy receptions over time, each plot representing an individual array. These data are $r e$-normalized such that the mean energy in each panel is unity; $\left\langle I_{f, \alpha}\right\rangle=1$. This is done so that we can more easily interpret results across a constant scale. In order to directly compare energy fluctuations at different angles, one can refer to the histograms shown on the right. Histogram data were not re- 
normalized, and are direct subsets of data shown in Figure 3.21. As a reference, the NIW sound speed profile is plotted below, and represents what the acoustic source is experiencing versus time. This profile is also valid for the absolutely parallel case $\left(\alpha=0^{\circ}\right)$ since it extends uniformly across range. However, it is not representative of what the other receiving VLAs experience, since they will observe a "shifted" version of the same profile. An asterisk is placed at the point where the first soliton reaches the VLA to help interpret this relative shift. Results for each $\alpha$ are expanded between Figure 3.23 and Figure 3.27.

Let us first examine the condition when the NIW reaches the acoustic source before the receiver. This corresponds to $\alpha=4^{\circ}, 2^{\circ}$; shown in the uppermost panels of Figure 3.22, with depth and modal dependence shown in Figure 3.23 and Figure 3.24. We observe a steady ramping of energy prior to the NIW's arrival at either the source or receiver. In the case of $\alpha=4^{\circ}$, the ramping is slight, increasing between $I_{f} \approx 2.5 \rightarrow 5$. However, it peaks sharply just prior to the first soliton reaching the acoustic source. This sharp high-energy reception stems from a defocusing event at the source, vectoring energy four degrees off-axis. In the case of $\alpha=2^{\circ}$, the energy escalation is more steady. In both instances, the final energy spike concluding this "ramp" is over ten-fold the average arrival. This is followed by a quelled period of vacillating energy that corresponds to the duration of strongest NIW activity. While one may expect the region of highest soliton activity to create the largest fluctuations, the receiver is located sufficiently off-axis and trapped energy is being completely vectored away from the array. Once the strongest solitons have passed, another brief period of energy arrives. 
For $\alpha=2^{\circ}$, it appears as a "reverse-ramp," due to a complicated back-end refraction interference pattern . In the reverse-ramp scenario, smaller solitons located towards the finale of the NIW train create weaker channels, spreading a portion of energy towards the larger solitons. This downrange energy is then refracted back into the smaller solitons, creating an interesting interference phenomenon. Following this, smaller perturbations towards the end of the NIW train continue to create smaller (but not insignificant) fluctuations. The histograms, mean, and variance for $\alpha=4^{\circ}, 2^{\circ}$ indicate that energy delivery on these arrays is slightly higher than the overall sample set, and the fluctuations are more severe. Interestingly, more energy and increased variability is received at $\alpha=4^{\circ}$ versus $\alpha=2^{\circ}$, because the effects of defocusing are more prominent at this slightly wider angle. Figure 3.23 and Figure 3.24 show the depth and modal dependence upon $I_{f}$ for these positive angles - where in both cases, the energy ramping feature is created by escalating energy within ducts, the strongest appearing at 70 meters depth. Modal dependence illustrates the ramp is caused by higher order modes, dominated by mode 3 and mode 4 , with modes $2-7$ significantly contributing. While mode 1 does not prove to be an important factor in this process, it does contribute to overall fluctuations when strong soliton activity dominates the entire acoustic track. This is to be expected, since mode 1 is not easily trapped within the horizontal sound channels.

Moving to the exactly parallel case, we notice a very different result for $\alpha=0^{\circ}$. After re-normalizing these simulated receptions, we see that refraction prior to the NIW event is of secondary importance when compared to energy fluctuations received during soliton activity. Dramatic spikes and fades occur that align well with individual soliton arrivals. 
As the strongest solitons pass, the fluctuations become less periodic, but are still substantial. Compared to the previous cases when $\alpha=4^{\circ}, 2^{\circ}$, the average energy upon the receiving VLA is 7-9 times the sample-set's reference value. Further, the signal variability is an order of magnitude higher. Figure 3.25 shows that refraction prior to the NIW's arrival at the acoustic track is again dominated by Modes 3 and 4, and in general, the higher order modes. This refraction shows the same structure as $\alpha=2^{\circ}$, indicating that this phenomenon is still important, yet may be over shadowed by the extreme energy fluctuations that occur during NIW activity.

Examining the case for $\alpha=-4^{\circ},-2^{\circ}$, we see strikingly similar patterns as $\alpha=4^{\circ}, 2^{\circ}$. In this instance, the NIW reaches the receiving VLA prior to the acoustic source. Again, the ramping feature indicates horizontal refraction prior to the NIW event is an important effect. In each instance a period of suppressed energy exists immediately after the ramp. This is because the leading soliton creates a barrier, shielding energy from the receiver until it has completely crossed the entire acoustic track. Once the NIW has passed both the source and receiver, fluctuations begin to appear. As in $\alpha=4^{\circ}, 2^{\circ}$, they are of smaller scale. For $\alpha=-2^{\circ}$, the back-end refraction effect appears once more. Mean energy and variability is similar to the previous off-axis conditions. Figure 3.26 and Figure 3.27 indicate the same reliance upon higher-order modes.

An additional, and often used, measure of signal variability is scintillation index

$$
S I_{\left(I_{f}\right)}=\frac{\left\langle I_{f}^{2}\right\rangle}{\left\langle I_{f}\right\rangle^{2}}-1,
$$


where scintillation index for modal energy $S I_{\left(A_{m f}\right)}$ would be calculated in identical fashion. Scintillation index is fundamentally a measure of signal variability, and is often associated with scattering, usually due to surface and bottom roughness. $S I>1$ is also referred to as a limit for saturation, ${ }^{46-49}$ where the pressure field is represented by an incoherent summation of random contributing wavefronts. Our concern is not with surface or bottom scattering, and therefore the PE model was not tailored to account for those factors. However, water-column inhomogeneity due to the NIW field, and energy transfer into the bottom, can cause severely distorted wavefronts and create large fluctuations in the acoustic field. In our case, for SI to breach unity, we require high intensity events to dominate a relatively low background energy. Scintillation index dependence for both $I_{f}$ and $A_{m f}$ is shown in Figure 3.28 versus angle (or cross-range). We see that as the acoustic track draws closer to exactly parallel, SI increases. Inside the $\pm 1^{\circ}$ domain, $S I$ is much greater than one, representing the sharply vacillating sound field the receiver experiences as repeated soliton waveguides pass the acoustic track.

\section{F Time-varying fluctuation regimes}

To compare the importance of refraction prior to the NIW's arrival relative to fluctuations during NIW activity, we can delineate the simulated time arrivals into timevarying fluctuation regimes. Based upon the dominant physical processes for each section of the modeled data, we define the time before the NIW reaches the sourcereceiver path as the refraction regime, and the time after the NIW has crossed the entire acoustic track the NIW interference regime. Figure 3.29 compares histograms for $\alpha=-4^{\circ},-2^{\circ}, 0^{\circ}, 2^{\circ}, 4^{\circ}$ (originally shown in Figure 3.22) to segments of data where 
refraction and NIW interference dominate. As an example, the top left panel shows the histogram for all data received at $\alpha=-4^{\circ}$. The top-center histogram represents the subset of these data defined by the refraction regime - this group corresponding to acoustic fluctuations in the unperturbed portion of the water column prior to the NIW's arrival. The top-right panel shows the influence of NIW interference effects, and constitutes simulated receptions after the leading soliton had crossed the entire acoustic track (the region beyond the asterisks in Figure 3.22). To compare energy differences between regimes, the lognormal and exponential mean and variance for each histogram is provided. While the overall data shown in Figure 3.21 is best fit by a lognormal distribution, certain subsets are better fit by an exponential distribution. Therefore, parameters for each type are provided. In some cases (i.e. refraction, $2^{\circ}$ off-axis), these data subsets appear to have bimodal distributions, with one peak near zero (similar to an exponential fit), and a secondary peak at higher energy (similar to a lognormal fit).

For both $\alpha=4^{\circ}$ and $\alpha=2^{\circ}$, the aggregate datasets possess comparable mean and variance parameters (left panels). However, important differences exist between them once they are separated into regimes. For $\alpha=4^{\circ}$, the refraction regime slightly dominates the NIW interference regime in both mean energy and variability delivered to the receiver. Moving to the case of $\alpha=2^{\circ}$, the trend is exaggerated. The reasoning for this difference is that while the slightly wider angle of $\alpha=4^{\circ}$ receives less refraction from the Lloyd's mirror effect early on in the NIW's passage, it is ideally located to receive redirected energy from defocusing as solitons pass over the acoustic source. Therefore NIW interference effects are greater for $\alpha=4^{\circ}$ when compared to $\alpha=2^{\circ}$. For the directly parallel case, $\alpha=0^{\circ}$, refraction is even stronger than when $\alpha=4^{\circ}, 2^{\circ}$, but is 
very much overshadowed by the undulating energy received during the NIW interference regime. Because focusing plays such a strong role in this instance, the tail of the histogram is elongated, and the data begins to resemble an exponential distribution. Moving on to $\alpha=-2^{\circ},-4^{\circ}$, similar trends as the positive off-axis angles exist.

\subsection{SUMMARY AND CONCLUSIONS}

In summary, we have implemented the Monterey- Miami Parabolic Equation algorithm to closely examine shallow water propagation when nonlinear internal waves dominate the sound field. Several modeling parameters were closely considered to ensure the accuracy of the model particular to this study, including source considerations, bottom parameters, the choice of reference sound speed, and a customized sound speed profile indicative of a real-world (yet general) scenario. We examined discrete instances where NIW activity creates refraction, defocusing, and focusing scenarios. Finally, we simulated a NIW traversing the acoustic field and used a statistical approach to (1) infer spatial differences by varying the acoustic track angle, and (2) temporal differences based upon time-varying fluctuation regimes.

To conclude, three-dimensional parabolic equation modeling has served as a highly informative tool to better-understand the complex problem of acoustic propagation in the presence of NIWs. The primary benefit of our modeling approach is that we have removed the element of oceanographic uncertainty that exists with measured field data. Our work is complementary to many other efforts in this area of study, and we expand it by further elaborating upon azimuthal dependencies that fall within the horizontal refraction and focusing regimes. ${ }^{10}$ In this situation, the difference between an acoustic 
track exactly parallel to a propagating NIW front versus being slightly askew is significant. In perfectly ideal conditions, during the exactly parallel case, focusing becomes a first order factor, and refraction prior to the NIW's arrival has secondary influence. We are not suggesting refraction prior to the NIW's arrival is unimportant - in fact, a slightly off-axis configuration will result in refraction effects that are similar to fluctuations during NIW activity, if not greater. Further, because the ideal circumstances are potentially less likely in real-world scenarios, refraction effects may be more commonplace in the shallow water environment. 


\section{ACKNOWLEDGEMENTS}

This work would have not been possible without support from the Office of Naval Research. The authors wish to graciously acknowledge ONR's continued backing and recognition of the shallow water acoustics community. We extend additional thanks to the hardworking individuals who supported the SW06 experiment, which served as the motivation for this work. Finally, this work would not have been possible without the help and resources from the University of Rhode Island Engineering and Computer Center. 


\section{REFERENCES AND LINKS}

1. $\quad$ F. D. Tappert, "The parabolic approximation method," Wave propagation and underwater acoustics, Lecture Notes in Physics 70, 224-287 (1977).

2. K. B. Smith and J. A. Colosi, "Effects of solitons on acoustic energy flow in three dimensions," J. Acoust Soc. Am. 124 (66), 1038-1057 (2008).

3. B. J. Sperry, J. F. Lynch, G. Gawarkiewicz, C.-S. Chiu and A. Newhall, "Characteristics of acoustic propagation to the eastern vertical line array receiver during the summer 1996 New England shelfbreak PRIMER experiment," IEEE J. Ocean. Eng. 28 (4), 729-749 (2003).

4. J. R. Apel, M. Badiey, C.-S. Chiu, S. Finette, R. Headrick, J. Kemp, J. F. Lynch, A. Newhall, M. H. Orr, B. H. Pasewark, D. Tielbuerger, A. Turgut, K. Von Der Heydt and S. Wolf, "An overview of the 1995 SWARM shallow-water internal wave acoustic scattering experiment," IEEE J. Ocean. Eng. 22 (3), 465-500 (1997).

5. J. F. Lynch, S. R. Ramp, C.-S. Chiu, T. Y. Tang, Y. J. Yang and J. A. Simmen, "Research highlights from the Asian Seas International Acoustics Experiment in the South China Sea," IEEE J. Ocean. Eng. 29 (4), 1067-1074 (2004).

6. P. H. Dahl, R. Zhang, J. H. Miller, L. R. Bartek, Z. Peng, S. R. Ramp, J. X. Zhou, C. S. Chiu, J. F. Lynch, J. A. Simmen and others, "Overview of results from the asian seas international acoustics experiment in the east china sea," IEEE J. Ocean. Eng. 29 (4), 920-928 (2005).

7. A. E. Newhall, T. F. Duda, K. Von Der Heydt, J. D. Irish, J. N. Kemp, S. A. Lerner, S. P. Liberatore, Y. T. Lin, J. F. Lynch, A. R. Maffei and others, "Acoustic and oceanographic observations and configuration information for the WHOI moorings from the SW06 experiment," WHOI technical report No. WHOI-2007-04 (2007).

8. J.-x. Zhou, X.-z. Zhang and P. H. Rogers, "Resonant interaction of sound wave with internal solitons in the coastal zone," J. Acoust. Soc. Am. 90 (4), 2042-2054 (1991). 
9. B. G. Katsnelson and S. A. Pereselkov, "Low-Frequency Horizontal Acoustic Refraction Caused by Internal Wave Solitons in a Shallow Sea.," Acoust. Phys. 46 (6), 684 (2000).

10. M. Badiey, B. G. Katsnelson, J. F. Lynch, S. Pereselkov and W. L. Siegmann, "Measurement and modeling of three-dimensional sound intensity variations due to shallow-water internal waves," J. Acoust. Soc. Am. 117 (2), 613-625 (2005).

11. M. Badiey, Y. Mu, J. Lynch, J. Apel and S. Wolf, "Temporal and azimuthal dependence of sound propagation in shallow water with internal waves," IEEE J. Ocean. Eng. 27 (1), 117-129 (2002).

12. T. F. Duda, "Examining the Validity of Approximations to Fully ThreeDimensional Shallow-Water Acoustic Propagation through Nonlinear Internal Gravity Waves," OCEANS 2007-Europe, 1-5 (2007).

13. S. Finette, M. H. Orr, A. Turgut, J. R. Apel, M. Badiey, C.-s. Chiu, R. H. Headrick, J. N. Kemp, J. F. Lynch, A. E. Newhall, K. v. Heydt, B. Pasewark, S. N. Wolf and D. Tielbuerger, "Acoustic field variability induced by time evolving internal wave fields," J. Acoust. Soc. Am. 108 (3), 957-972 (2000).

14. S. D. Frank, M. Badiey, J. F. Lynch and W. L. Siegmann, "Analysis and modeling of broadband airgun data influenced by nonlinear internal waves," J. Acoust. Soc. Am. 116 (6), 3404-3422 (2004).

15. S. D. Frank, M. Badiey, J. F. Lynch and W. L. Siegmann, "Experimental evidence of three-dimensional acoustic propagation caused by nonlinear internal waves," J. Acoust. Soc. Am. 118 (2), 723-734 (2005).

16. R. Oba and S. Finette, "Acoustic propagation through anisotropic internal wave fields: Transmission loss, cross-range coherence, and horizontal refraction," J. Acoust. Soc. Am. 111 (2), 769-784 (2002).

17. D. B. Reeder, T. F. Duda and B. Ma, "Short-Range Acoustic Propagation Variability on a Shelf Area with Strong Nonlinear Internal Waves," (2008).

18. M. Badiey, B. G. Katsnelson, Y. T. Lin and J. F. Lynch, "Acoustic multipath arrivals in the horizontal plane due to approaching nonlinear internal waves," J. Acoust. Soc. Am. 129 (4) (2011). 
19. Y.-T. Lin, T. F. Duda and J. F. Lynch, "Acoustic mode radiation from the termination of a truncated nonlinear internal gravity wave duct in a shallow ocean area," J. Acoust. Soc. Am. 126 (4), 1752-1765 (2009).

20. J. F. Lynch, Y. T. Lin, T. F. Duda and A. E. Newhall, "Acoustic Ducting, Reflection, Refraction, and Dispersion by Curved Nonlinear Internal Waves in Shallow Water," IEEE J. Ocean. Eng. 35 (1), 12-27 (2010).

21. M. Badiey, B. G. Katsnelson, J. F. Lynch and S. Pereselkov, "Frequency dependence and intensity fluctuations due to shallow water internal waves," J. Acoust. Soc. Am. 122 (2), 747-760 (2007).

22. J. Luo, M. Badiey, E. A. Karjadi, B. Katsnelson, A. Tskhoidze, J. F. Lynch and J. N. Moum, "Observation of sound focusing and defocusing due to propagating nonlinear internal waves," J. Acoust. Soc. Am. 124 (3), 66 (2008).

23. K. B. Smith, "Convergence, Stability, and Variability of Shallow Water Acoustic Predictions Using a Split-Step Fourier Parabolic Equation Model.," Journal of Computational Acoustics 9 (1), 243 (2001).

24. K. B. Smith and F. D. Tappert, "Horizontal refraction and the uncoupled azimuth approximation," 343 (2004).

25. D. J. Thomson and N. R. Chapman, "A wide-angle split-step algorithm for the parabolic equation," J. Acoust. Soc. Am. 93 (2627) (1993).

26. D. J. Thomson and C. S. Bohun, "A wide-angle initial field for the parabolic equation models," J. Acoust. Soc. Am 83 (S118) (1988).

27. C. T. Tindle and Z. Y. Zhang, "An equivalent fluid approximation for a low shear speed ocean bottom," J. Acoust. Soc. Am. 91 (6), 3248-3256 (1992).

28. Y.-M. Jiang, N. R. Chapman and M. Badiey, "Quantifying the uncertainty of geoacoustic parameter estimates for the New Jersey shelf by inverting air gun data," J. Acoust. Soc. Am. 121 (4), 1879-1894 (2007).

29. G. R. Potty, J. H. Miller, P. S. Wilson, J. F. Lynch and A. Newhall, "Geoacoustic inversion using combustive sound source signals," J. Acoust. Soc. Am. 124 (3), 146 (2008). 
30. D. J. Thomson and N. R. Chapman, "A wide-angle split-step algorithm for the parabolic equation," The Journal of the Acoustical Society of America 74 (6), 1848-1854 (1983).

31. F. D. Tappert and M. G. Brown, "Asymptotic phase errors in parabolic approximations to the one-way Helmholtz equation," The Journal of the Acoustical Society of America 99 (3), 1405-1413 (1996).

32. F. Tappert, J. L. Spiesberger and L. Boden, "New full-wave approximation for ocean acoustic travel time predictions," The Journal of the Acoustical Society of America 97 (5), 2771-2782 (1995).

33. A. R. Smith and K. B. Smith, "Mode functions for the wide-angle approximation to the parabolic equation," The Journal of the Acoustical Society of America 103 (2), 814-821 (1998).

34. D. B. K. S. A. Chin-Bing, J. A. Davis, R. B. Evans, in NRL Book Contribution (Naval Research Laboratory - Stennis Space Center, 1993).

35. M. B. Porter, "The KRAKEN normal mode program," Technical report, SACLANT Undersea Research Center (1992).

36. F. B. Jensen, W. A. Kuperman, M. B. Porter and H. Schmidt, "Computational Ocean Acoustics, AIP series in modern acoustics and signal processing," American Institute of Physics, New York (1994).

37. K. B. Smith, M. A. Wolfson and A. V. van Leijen, "Correction to attenuation treatment in the Monterey-Miami Parabolic Equation Model," Naval Postgraduate School Technical Report (2007).

38. K. V. Mackenzie, "Nine-term equation for sound speed in the oceans," J. Acoust. Soc. Am. 70 (3), 807-812 (1981).

39. D. Gong, J. T. Kohut and S. M. Glenn, "Seasonal climatology of wind-driven circulation on the New Jersey Shelf," J. Geophys. Res. 115 (C4), 04006 (2010).

40. J. F. Lynch, J. A. Colosi, G. G. Gawarkiewicz, T. F. Duda, A. D. Pierce, M. Badiey, B. G. Katsnelson, J. E. Miller, W. Siegmann, C.-S. Chiu and A. Newhall, 
"Consideration of fine-scale coastal oceanography and 3-D acoustics effects for the ESME sound exposure model," IEEE J. Ocean. Eng. 31 (1), 33-48 (2006).

41. R. H. Ferris, "Comparison of Measured and Calculated Normal-Mode Amplitude Functions for Acoustic Waves in Shallow Water," The Journal of the Acoustical Society of America 52 (3B), 981-989 (1972).

42. J. A. Colosi, "Acoustic mode coupling induced by shallow water nonlinear internal waves: Sensitivity to environmental conditions and space-time scales of internal waves," J. Acoust. Soc. Am. 124 (3), 1452-1464 (2008).

43. T. F. Duda, "Acoustic mode coupling by nonlinear internal wave packets in a shelfbreak front area," IEEE J. Ocean. Eng. 29 (1), 118-125 (2004).

44. X. Tang, F. D. Tappert and D. B. Creamer, "Simulations of large acoustic scintillations in the Straits of Florida," J. Acoust. Soc. Am. 120 (6), 3539-3552 (2006).

45. A. Fredricks, J. A. Colosi, J. F. Lynch, G. Gawarkiewicz, C.-S. Chiu and P. Abbot, "Analysis of multipath scintillations from long range acoustic transmissions on the New England continental slope and shelf," J. Acoust. Soc. Am. 117 (3), 1038-1057 (2005).

46. J. A. Colosi and A. B. Baggeroer, "On the kinematics of broadband multipath scintillation and the approach to saturation," J. Acoust. Soc. Am. 116 (6), 35153522 (2004).

47. D. B. Creamer, "Scintillating shallow-water waveguides," J. Acoust. Soc. Am. 99 (5), 2825-2838 (1996).

48. I. Dyer, "Statistics of Sound Propagation in the Ocean," J. Acoust. Soc. Am. 48 (1B), 337-345 (1970).

49. S. M. Flatte, R. Dashen, W. H. Munk, K. M. Watson and F. Zachariasen, Sound transmission through a fluctuating ocean. (Cambridge University Press, Cambridge, 1979). 


\section{FIGURES}
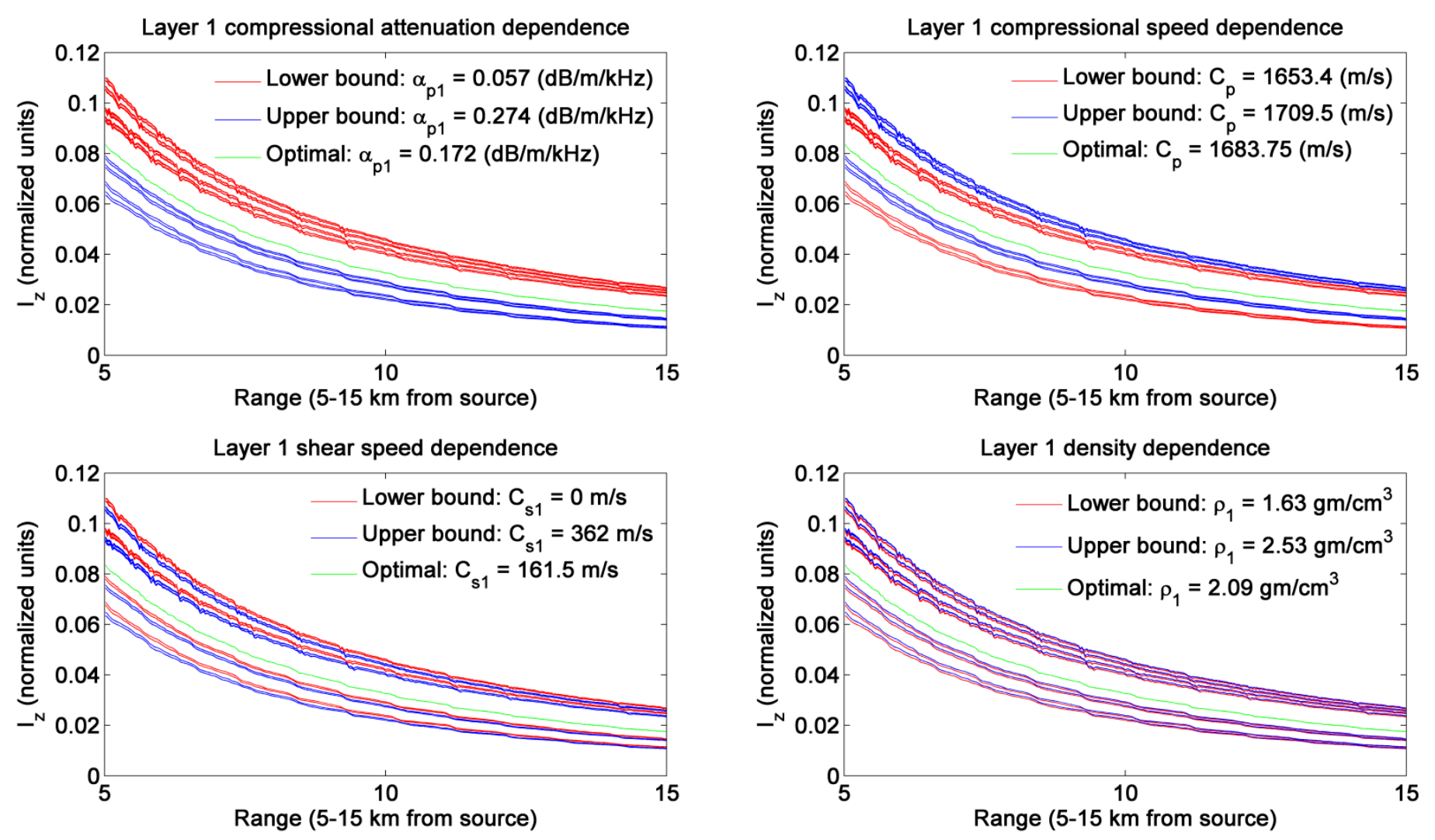

Figure 3.1 Over 1,000 two-dimensional PE simulations varied several bottom parameters based upon estimates from previously published inversion values. Depth-integrated intensity versus range shows the influence of these parameters upon bottom attenuation. Four distinct bands became apparent - indicating layer one compressional attenuation, compressional speed, shear speed, and density are the most influential factors. 

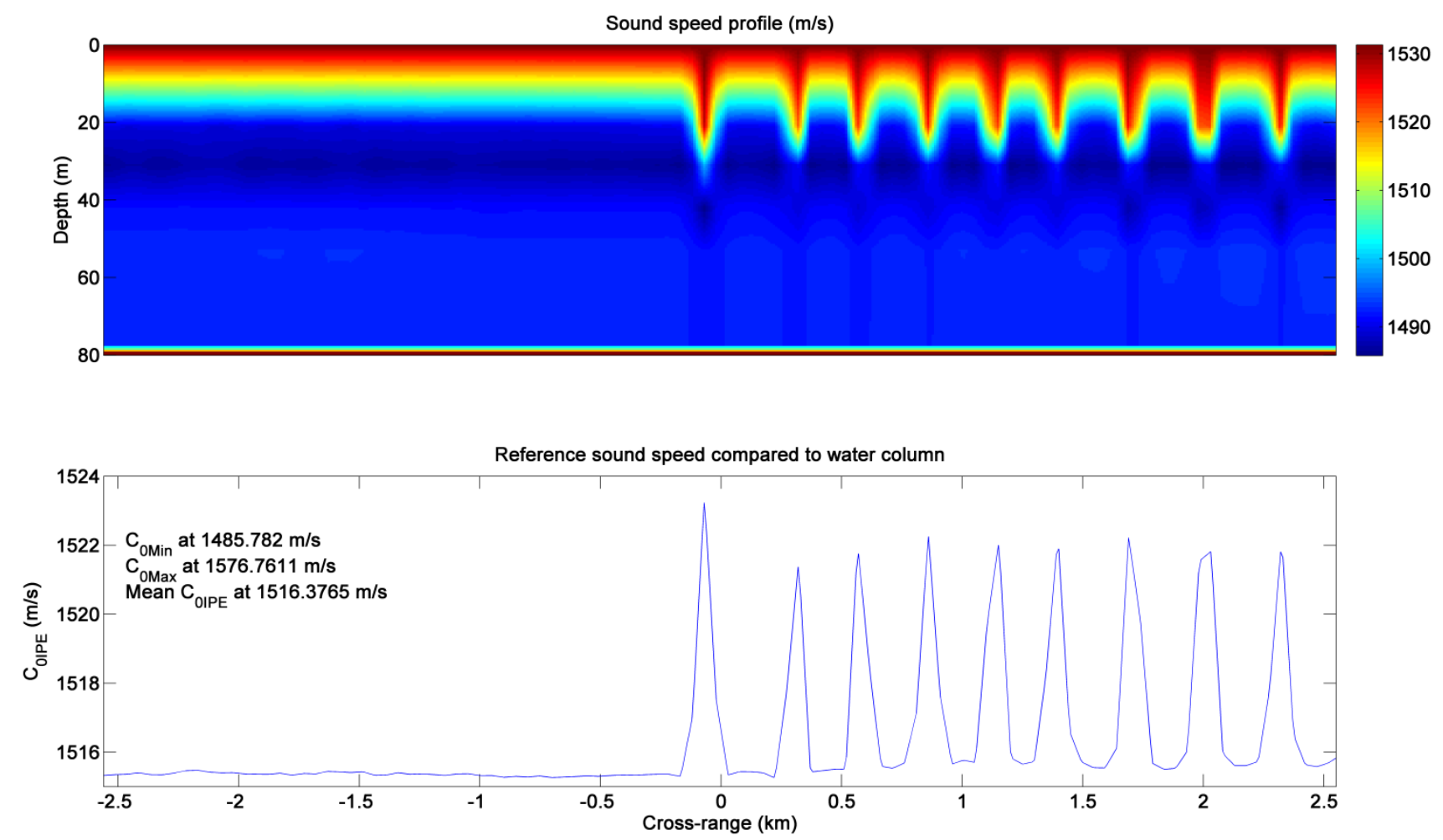

Figure 3.2 The choice of reference sound speed $\left(c_{0}\right)$ can impact the performance of the PE model. The top panel shows a modeled area where roughly half of the input profile contains a NIW, and where refraction effects will dominate. Below is the reference sound speed that would be associated with the $c_{0}$-independent $\left(c_{0 I P E}\right)$ calculation. 


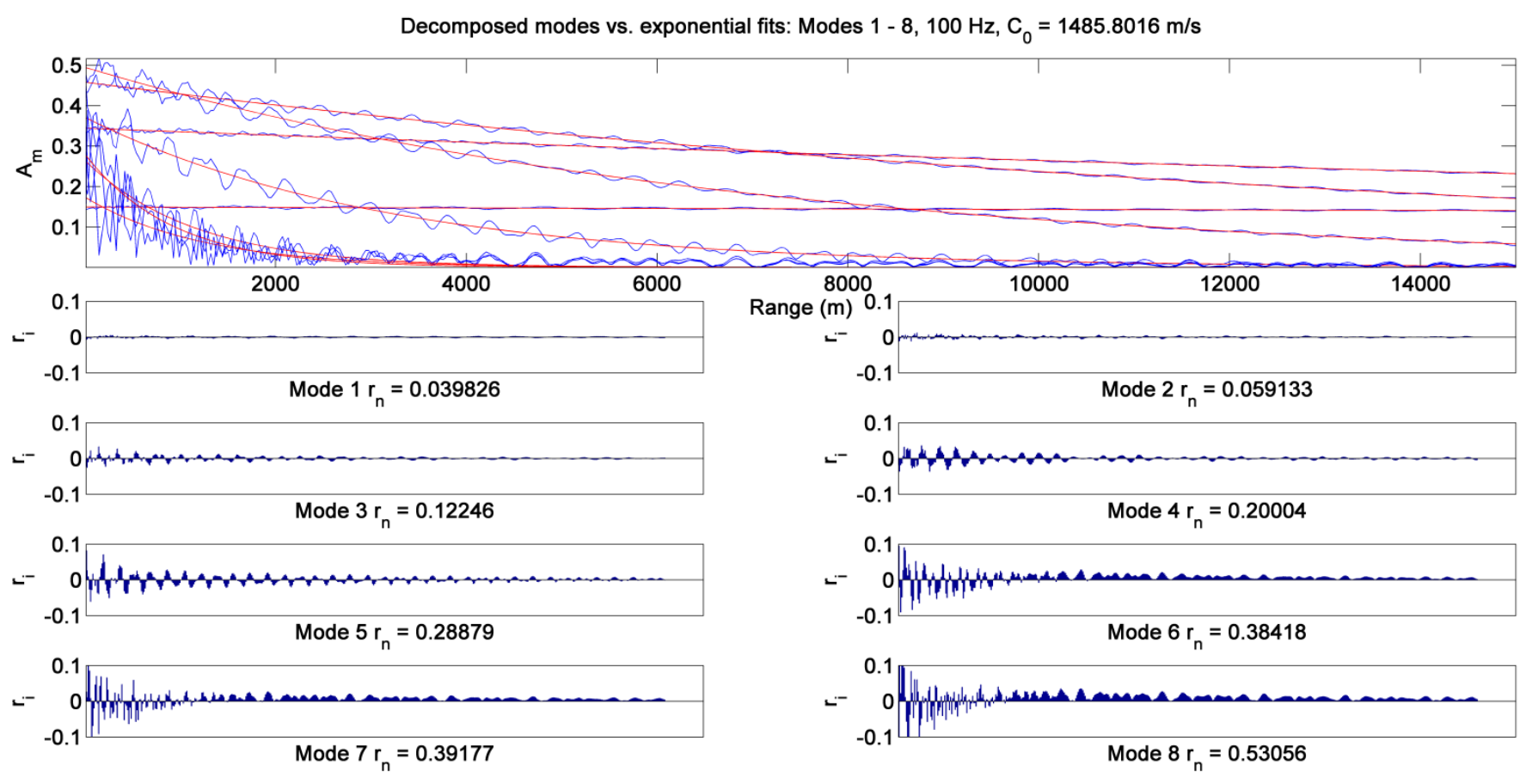

Figure 3.3 A sensitivity analysis compared the impact of varying $c_{0}$ upon modal decomposition. The choice of $c_{0}$ can more closely align eigenvalues of the Helmholtz equation with those of the wide angle source PE field. Modal decomposition of the PE field is shown in blue, with predicted in red (top). The residuals were used as a measure for goodness of fit. 
Three-dimensional acoustic problem for consideration

Progressing nonlinear internal wave train, near parallel acoustic path

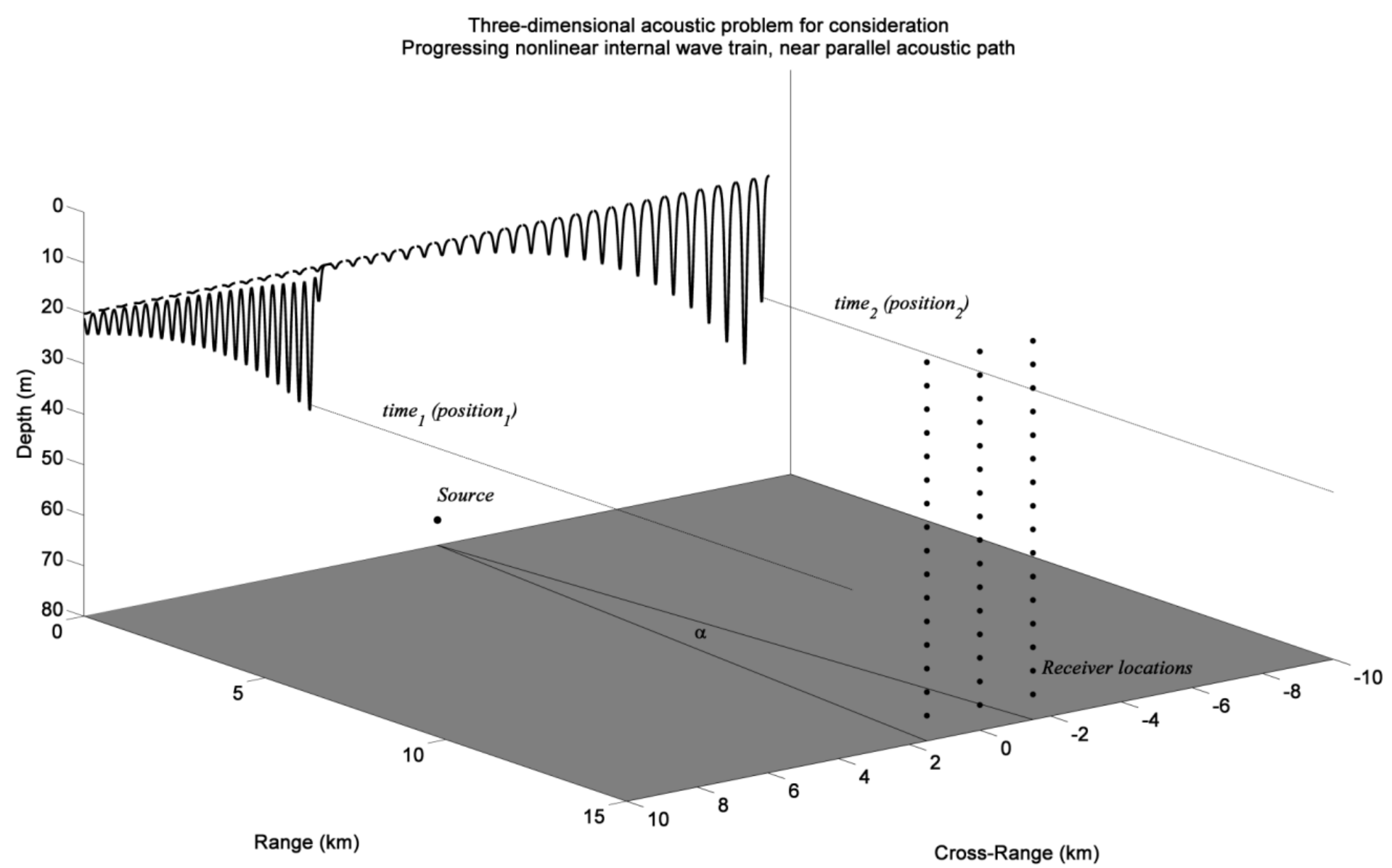

Figure 3.4 A general layout for the acoustic problem of interest. A progressing NIW approaches and crosses the acoustic track such that the soliton wave-fronts are nearly parallel. Horizontal refraction plays a dominant role in this scenario. 


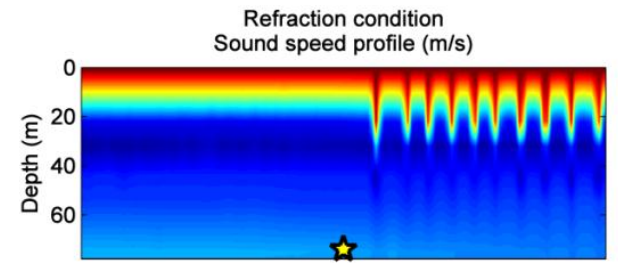

$100 \mathrm{~Hz}$ Depth Integrated Intensity, $\mathrm{I}_{\mathrm{z}}$ (dB re $1 \mathrm{~m}$ )
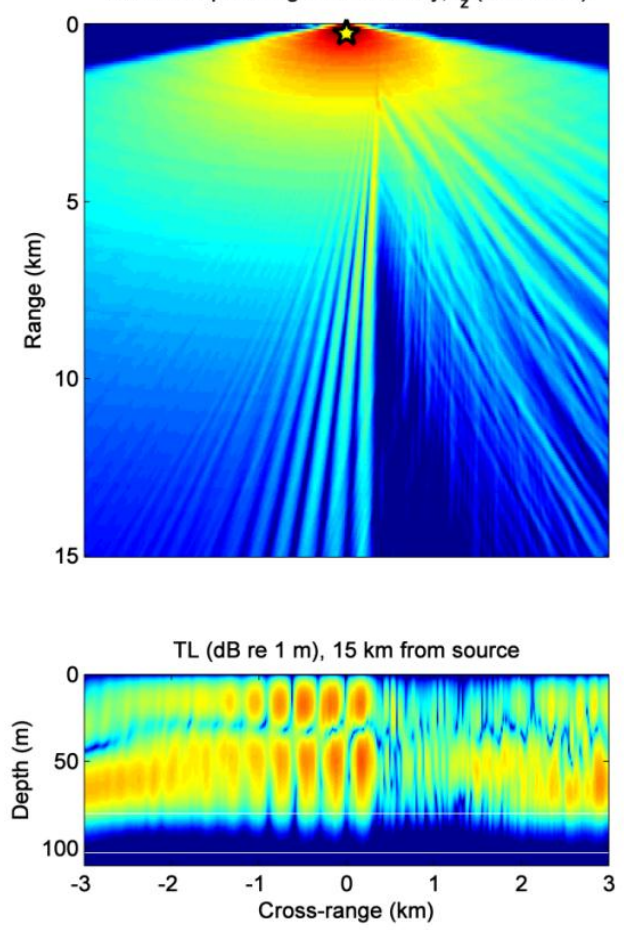

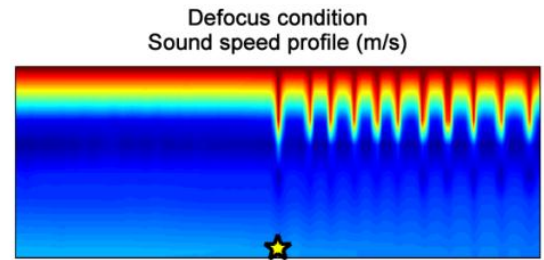

$100 \mathrm{~Hz}$ Depth Integrated Intensity, $I_{z}$ (dB re 1 m)

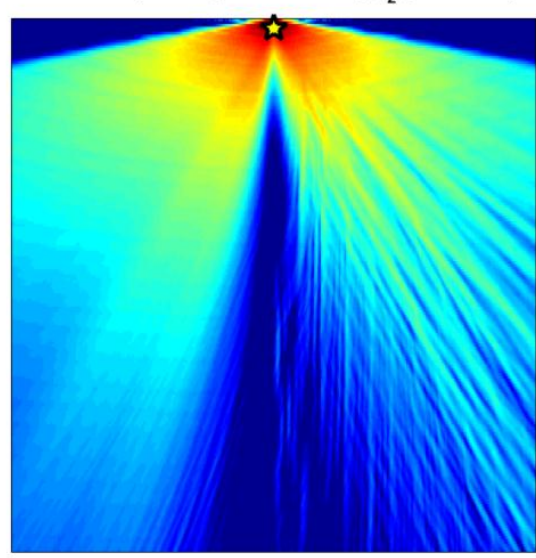

TL (dB re $1 \mathrm{~m}), 15 \mathrm{~km}$ from source

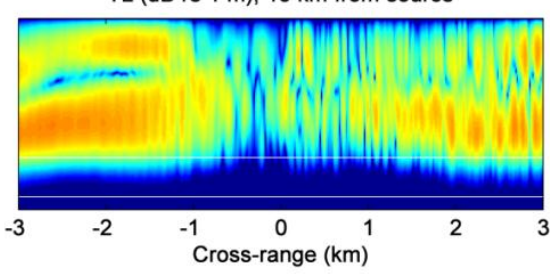

Focus condition
(

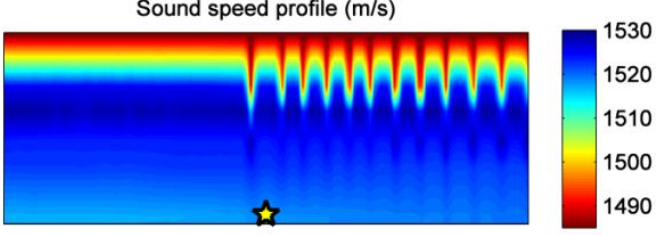

$100 \mathrm{~Hz}$ Depth Integrated Intensity, I (dB re $1 \mathrm{~m}$ )

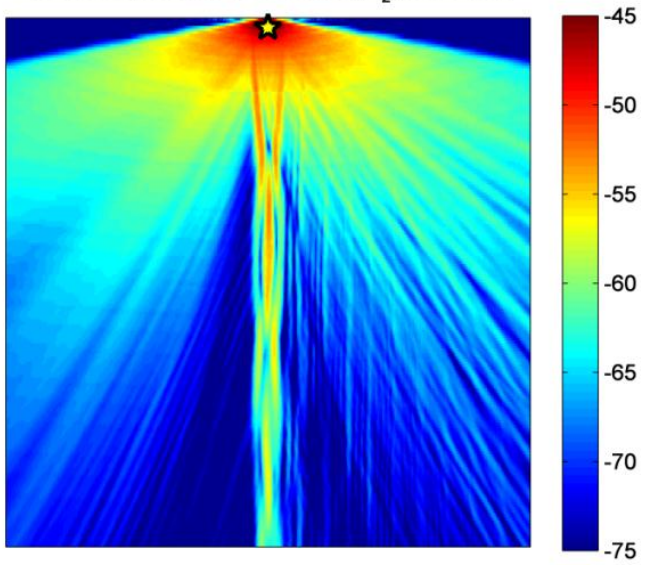

TL (dB re $1 \mathrm{~m}), 15 \mathrm{~km}$ from source

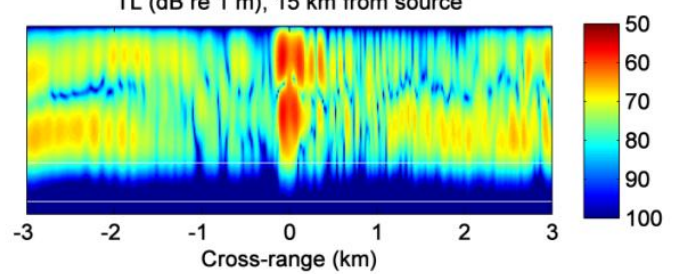

Figure 3.5 Three scenarios showing refraction (left), defocusing (center), and focusing (right). Top panels show the sound speed profile relative to the $100 \mathrm{~Hz}$ acoustic source (marked by a star) used in the PE model; the profile is valid across range. The center panels show depth integrated intensity throughout the three-dimensional water column. The bottom panels show transmission loss versus depth $15 \mathrm{~km}$ away from the acoustic source. (color online) 

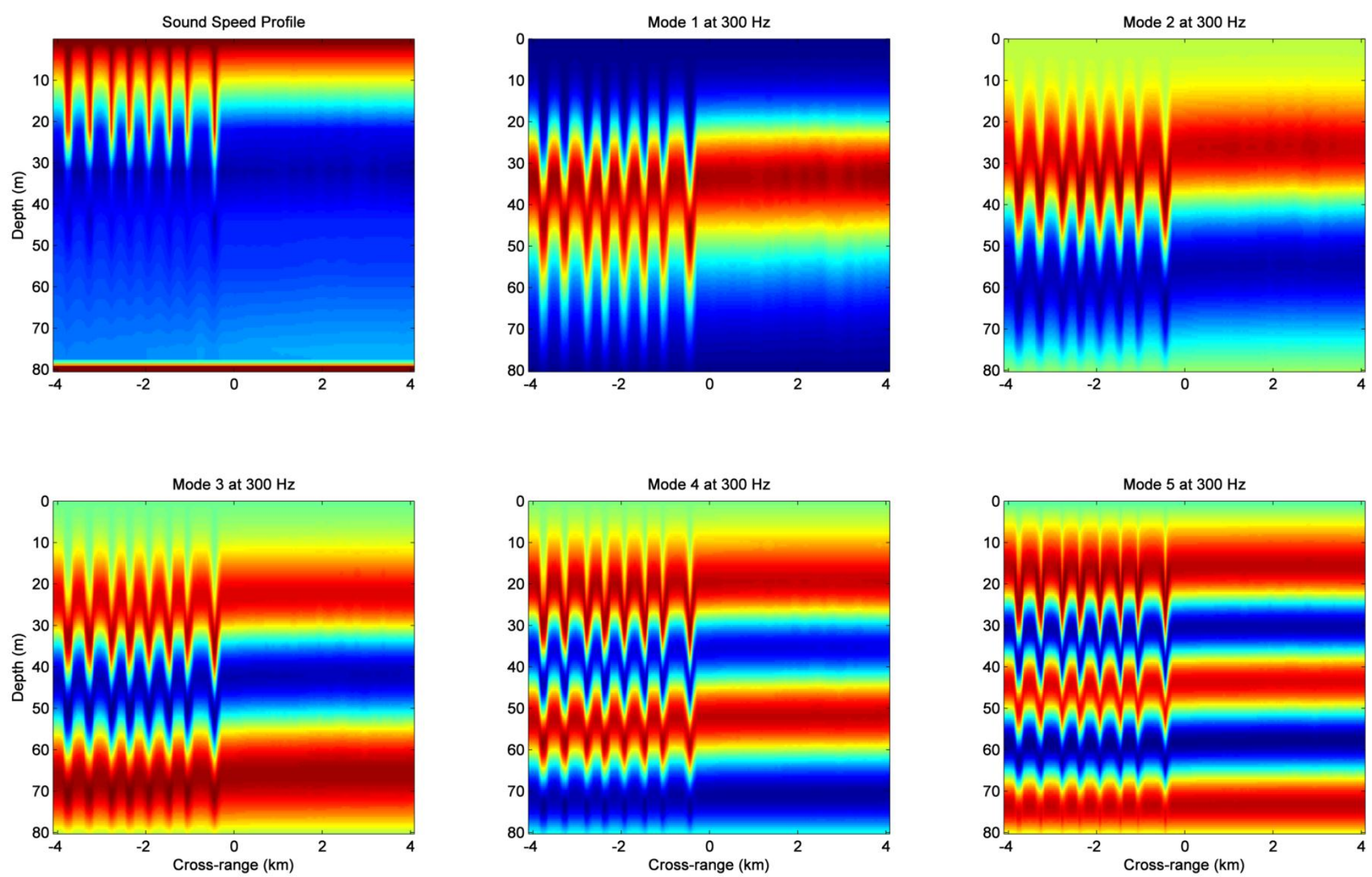

Figure 3.6 Modeshapes (for modes 1-5) at each cross-range point in the sound speed profile. The modeshape maxima and minima are pushed downward in the water column at soliton locations. (color online) 

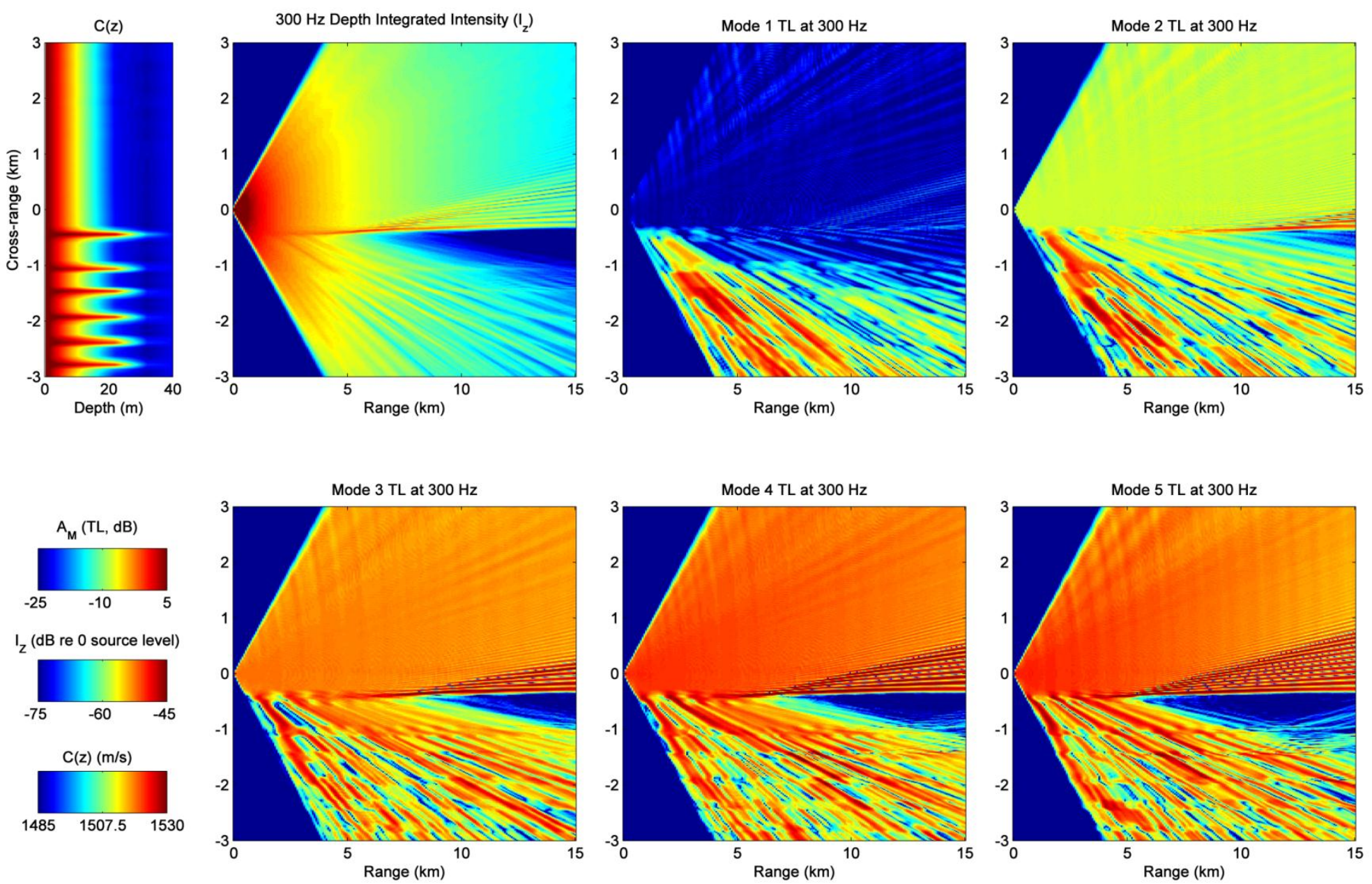

Figure 3.7 Depth integrated intensity and modal decomposition of the PE field for modes 1-5 for a $300 \mathrm{~Hz}$ source. Sound speed input creates a refraction scenario. (color online) 

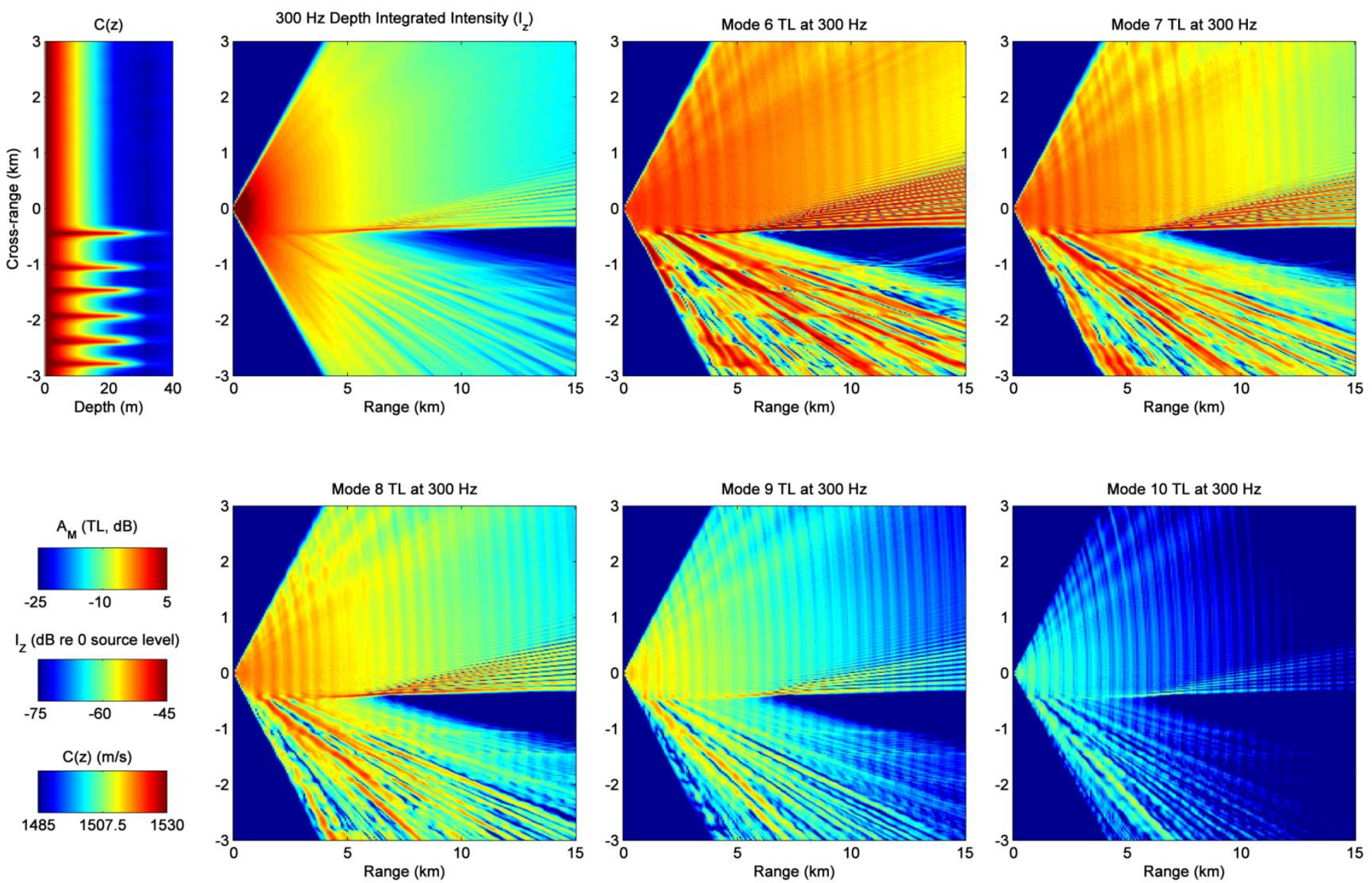

Figure 3.8 Depth integrated intensity and modal decomposition of the PE field for modes 6-10 for a $300 \mathrm{~Hz}$ source. Sound speed input creates a refraction scenario. (color online) 

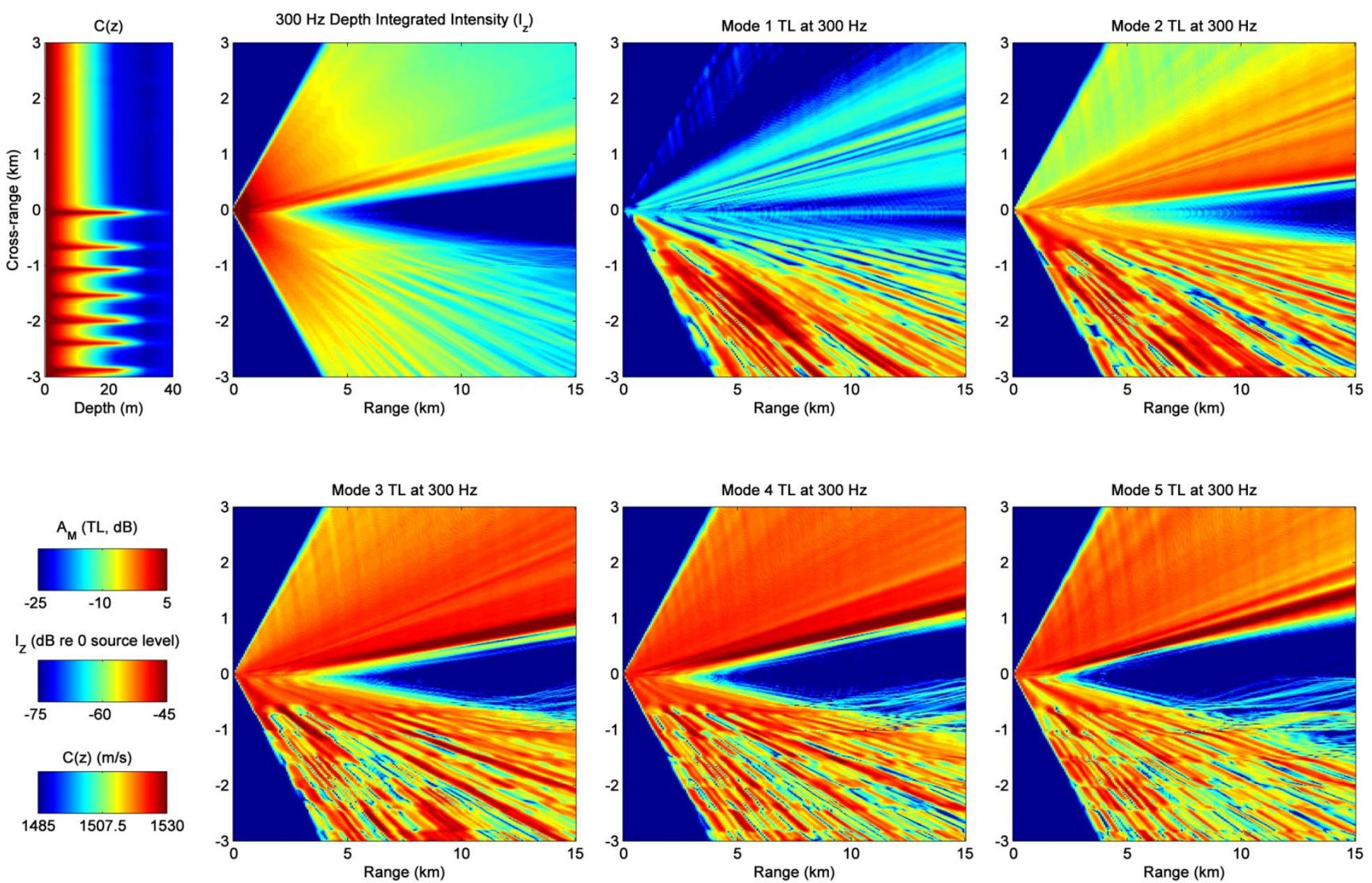

Figure 3.9 Depth integrated intensity and modal decomposition of the PE field for modes 1-5 for a $300 \mathrm{~Hz}$ source. Sound speed input creates defocus scenario. (color online) 

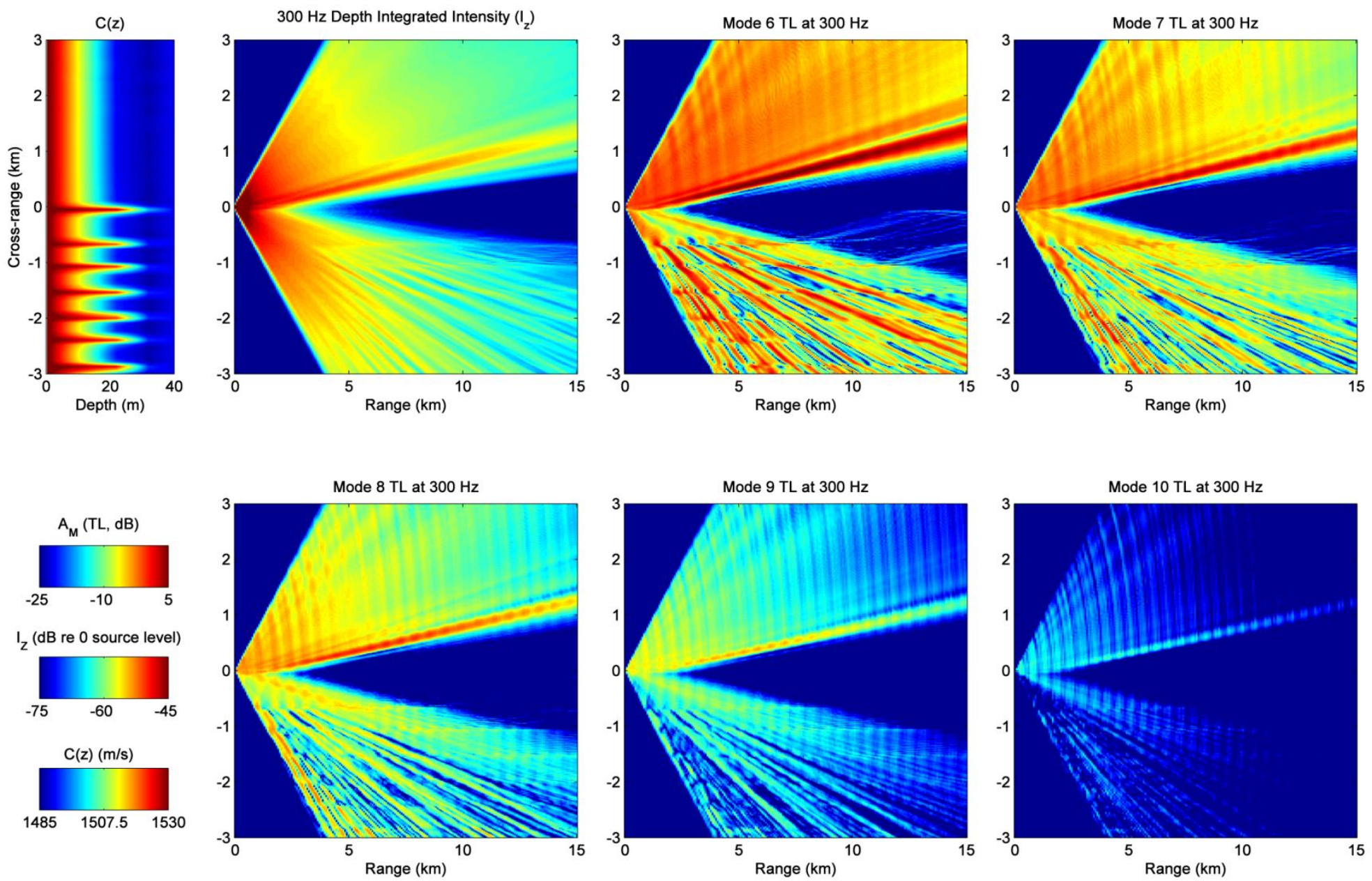

Figure 3.10 Depth integrated intensity and modal decomposition of the PE field for modes 6-10 for a $300 \mathrm{~Hz}$ source. Sound speed input creates defocus scenario. (color online) 

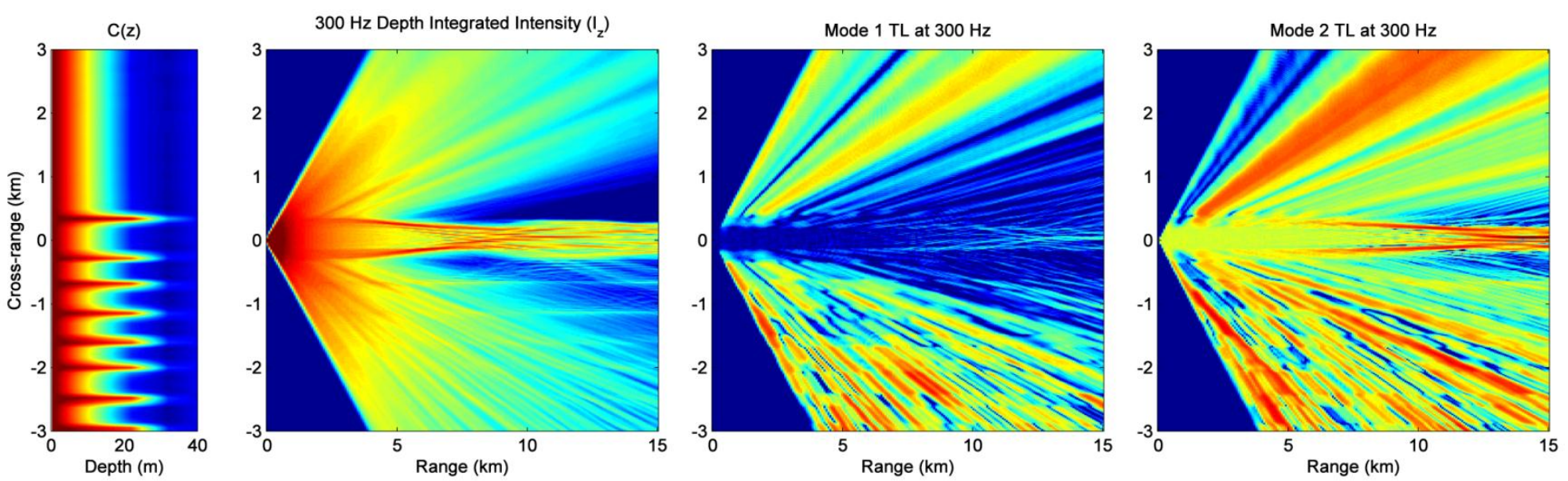

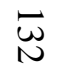
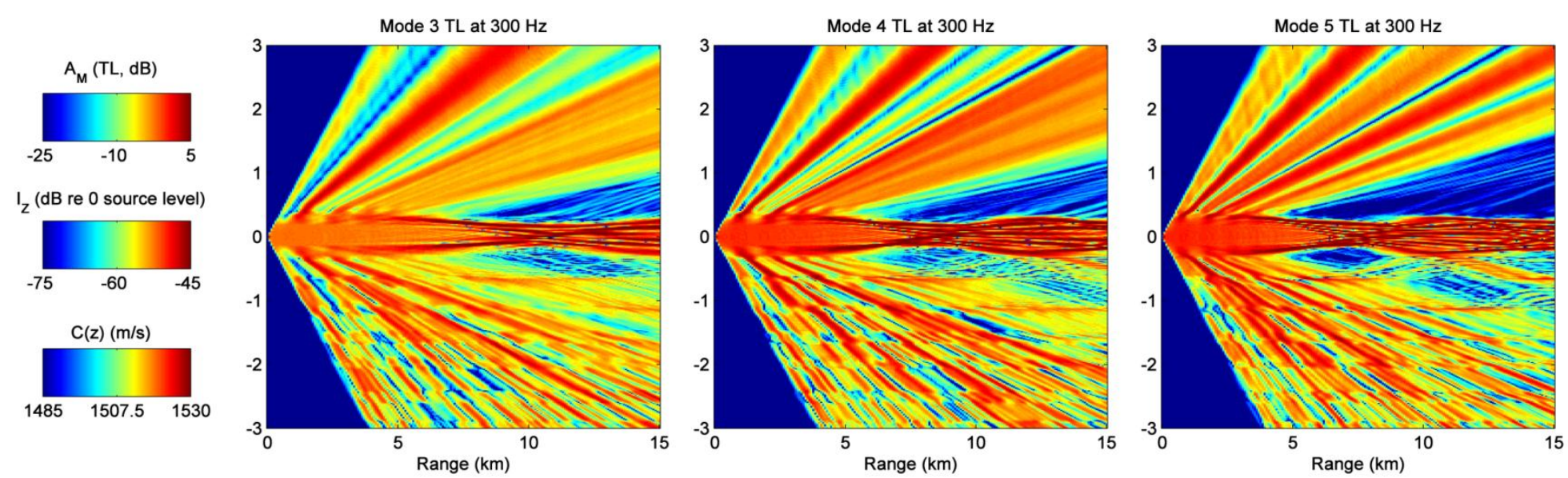

Figure 3.11 Depth integrated intensity and modal decomposition of the PE field for modes 1-5 for a $300 \mathrm{~Hz}$ source. Sound speed input creates a focus scenario. (color online) 

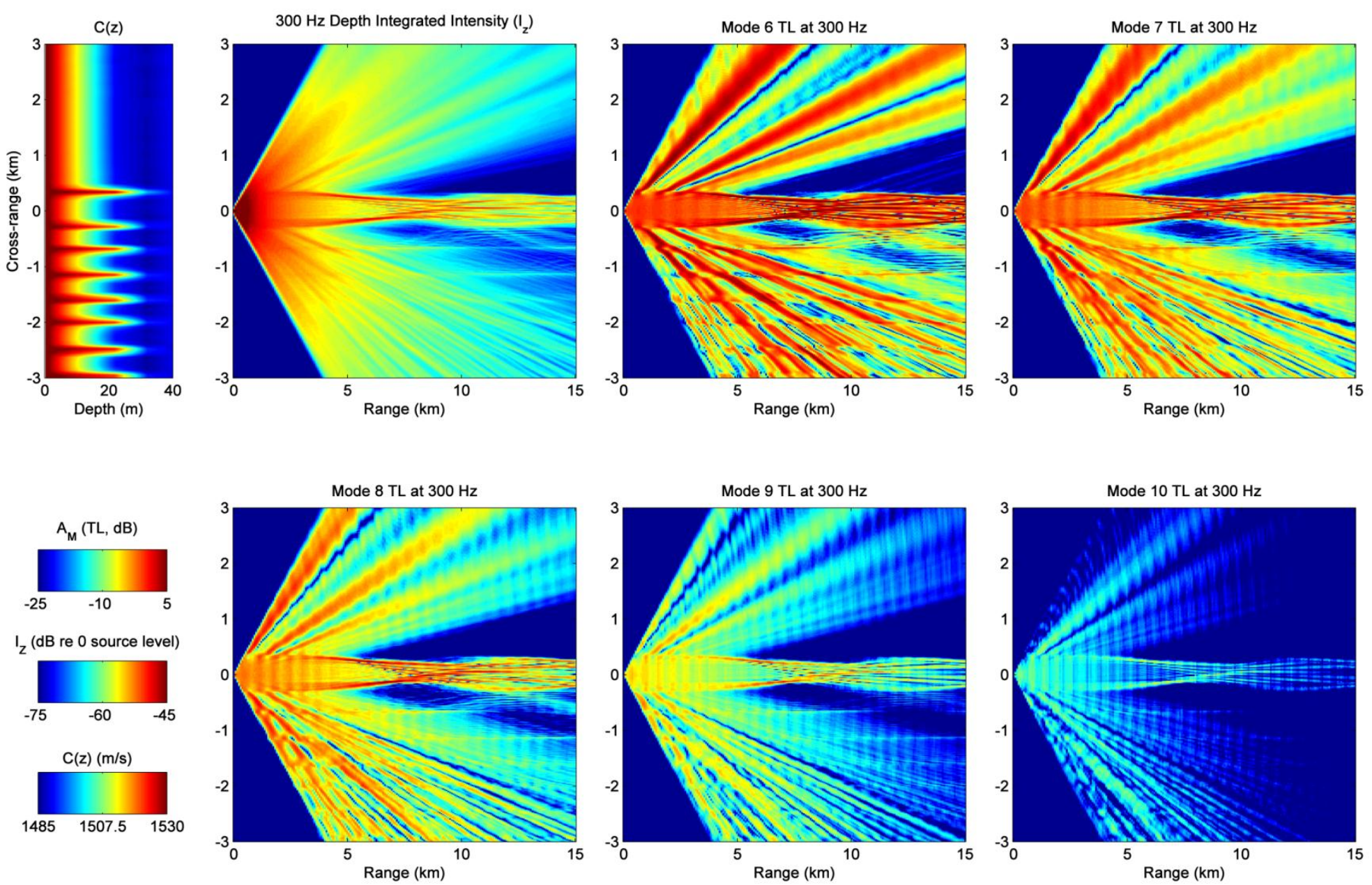

Figure 3.12 Depth integrated intensity and modal decomposition of the PE field for modes 6-10 for a 300 Hz source. Sound speed input creates a focus scenario. (color online) 
Sound speed profile - Refraction scenario
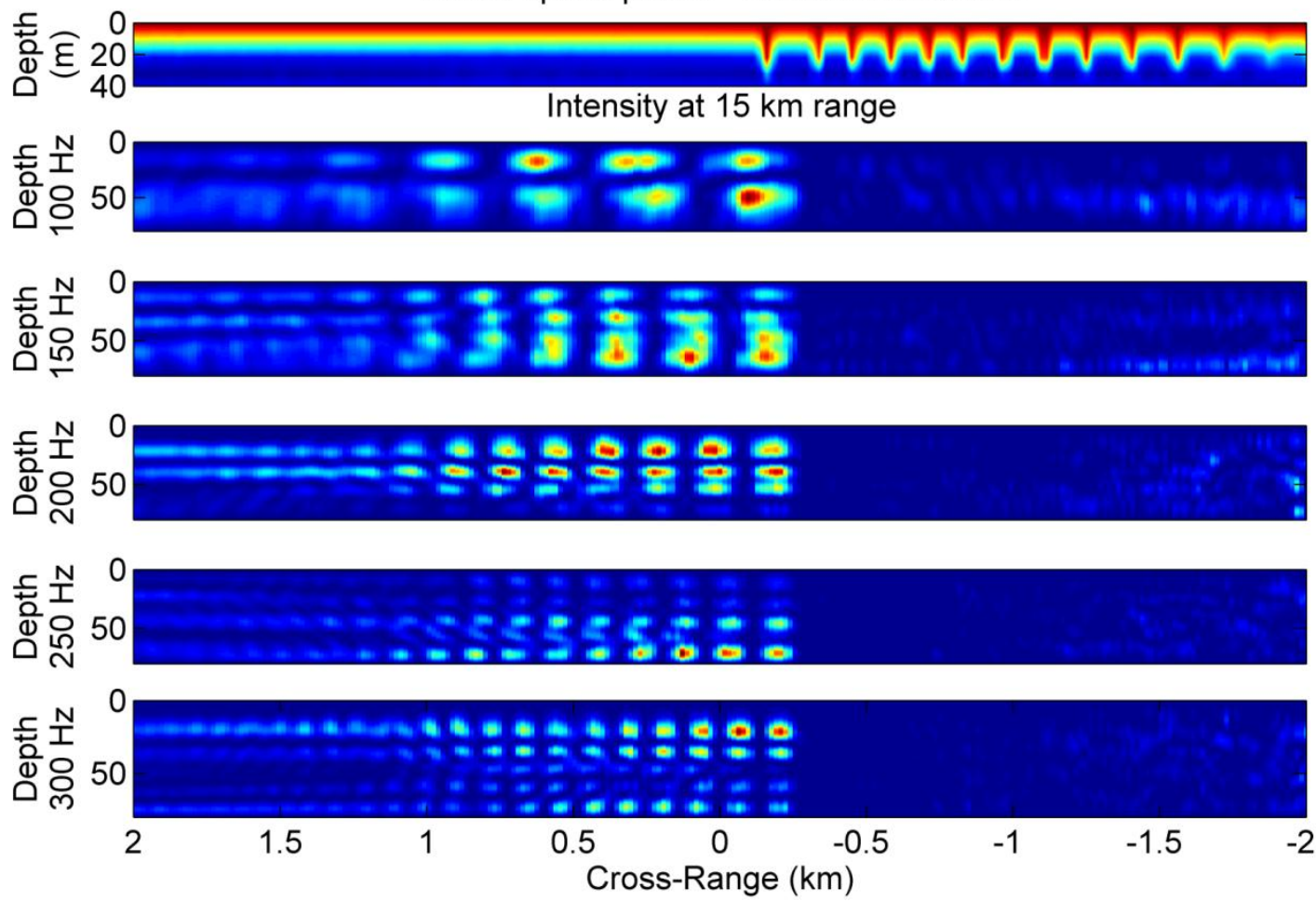

Figure 3.13 Frequency dependence during a refraction scenario. The sound speed input is valid across range (top). Normalized intensity at $15 \mathrm{~km}$ range for various frequencies (lower panels). (color online) 

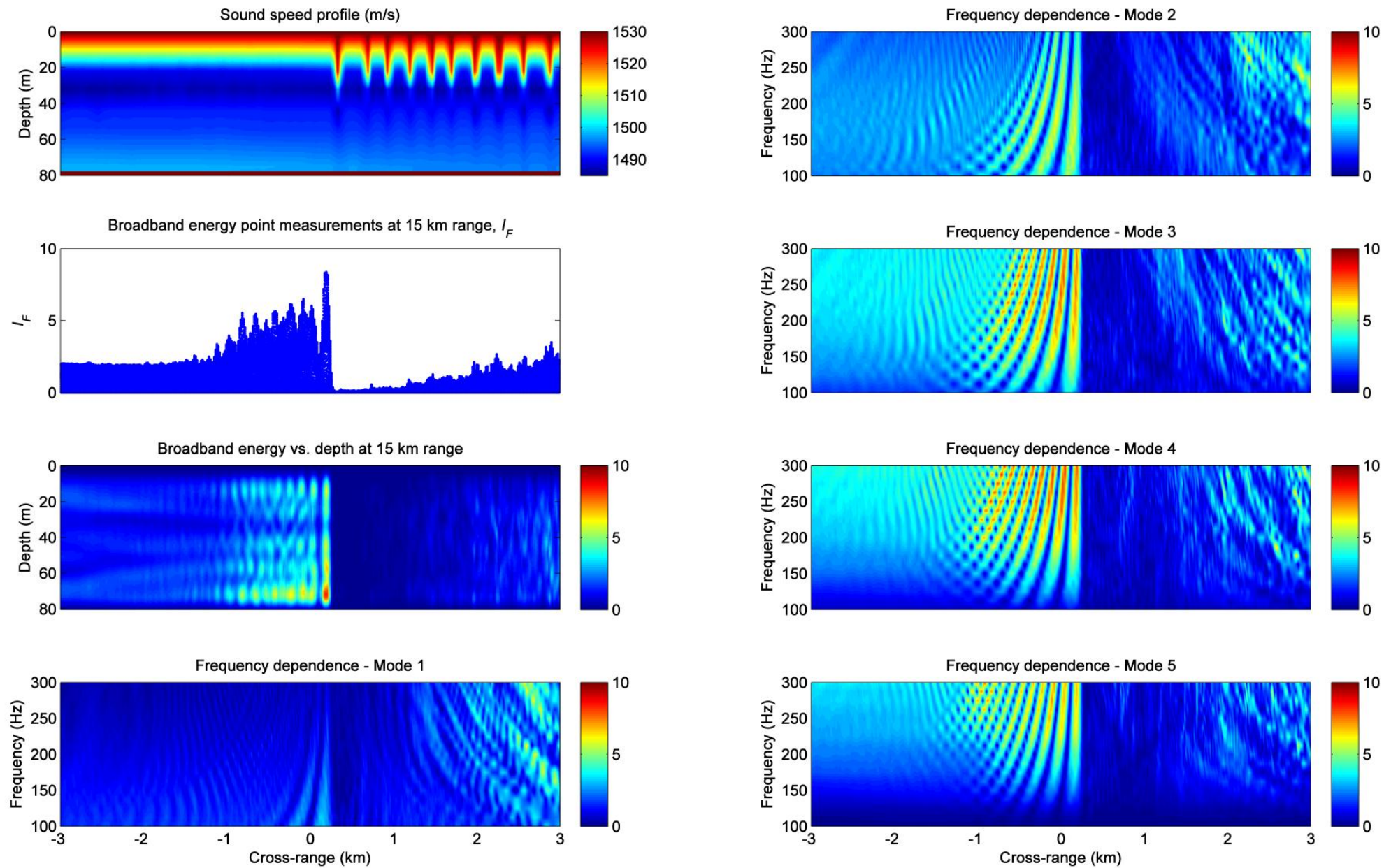

Figure 3.14 Broadband modal energy during a refraction condition is compared to modal decomposition of the PE field. Modal decomposition plots show mode amplitude at $15 \mathrm{~km}$ versus cross-range and frequency. (color online) 

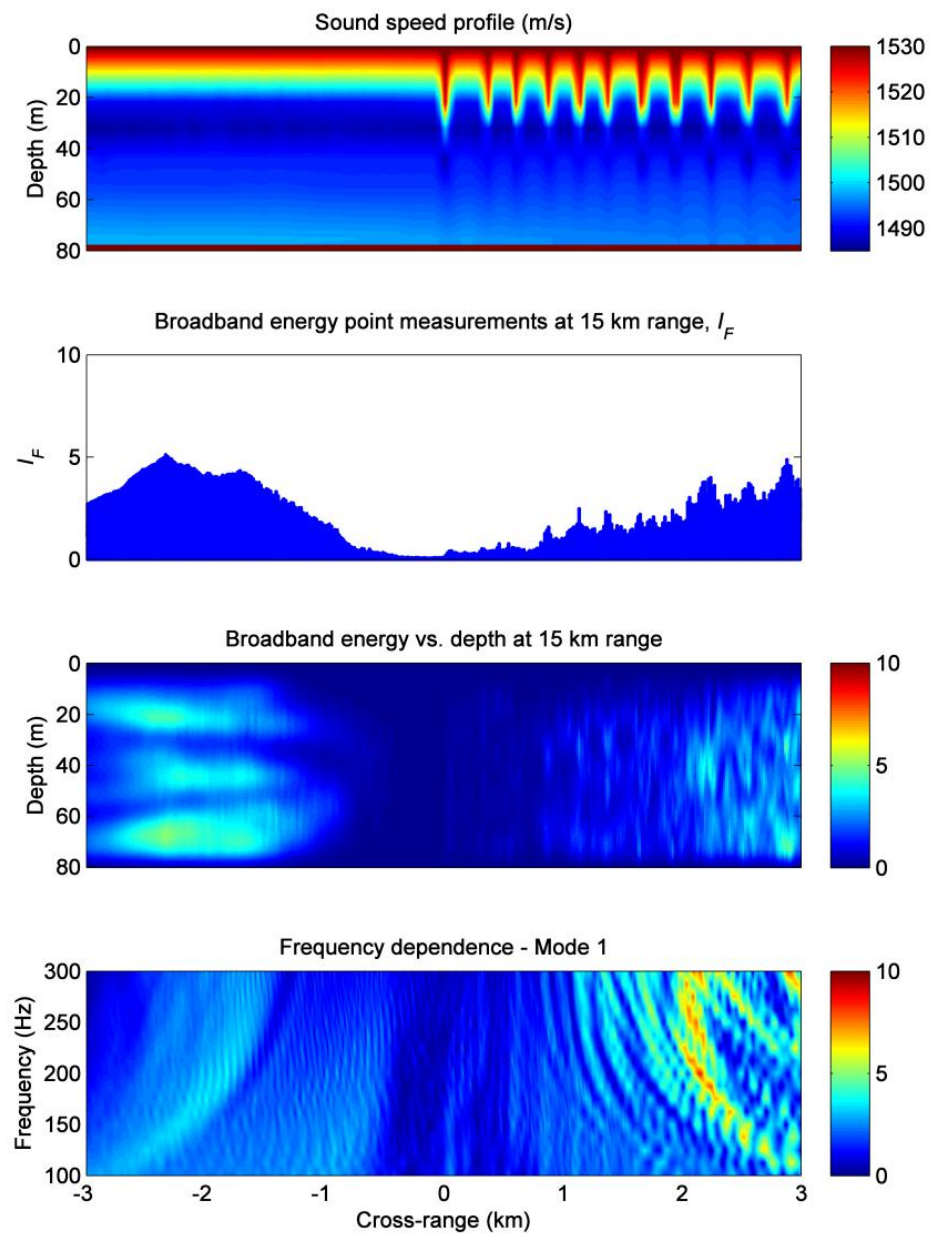
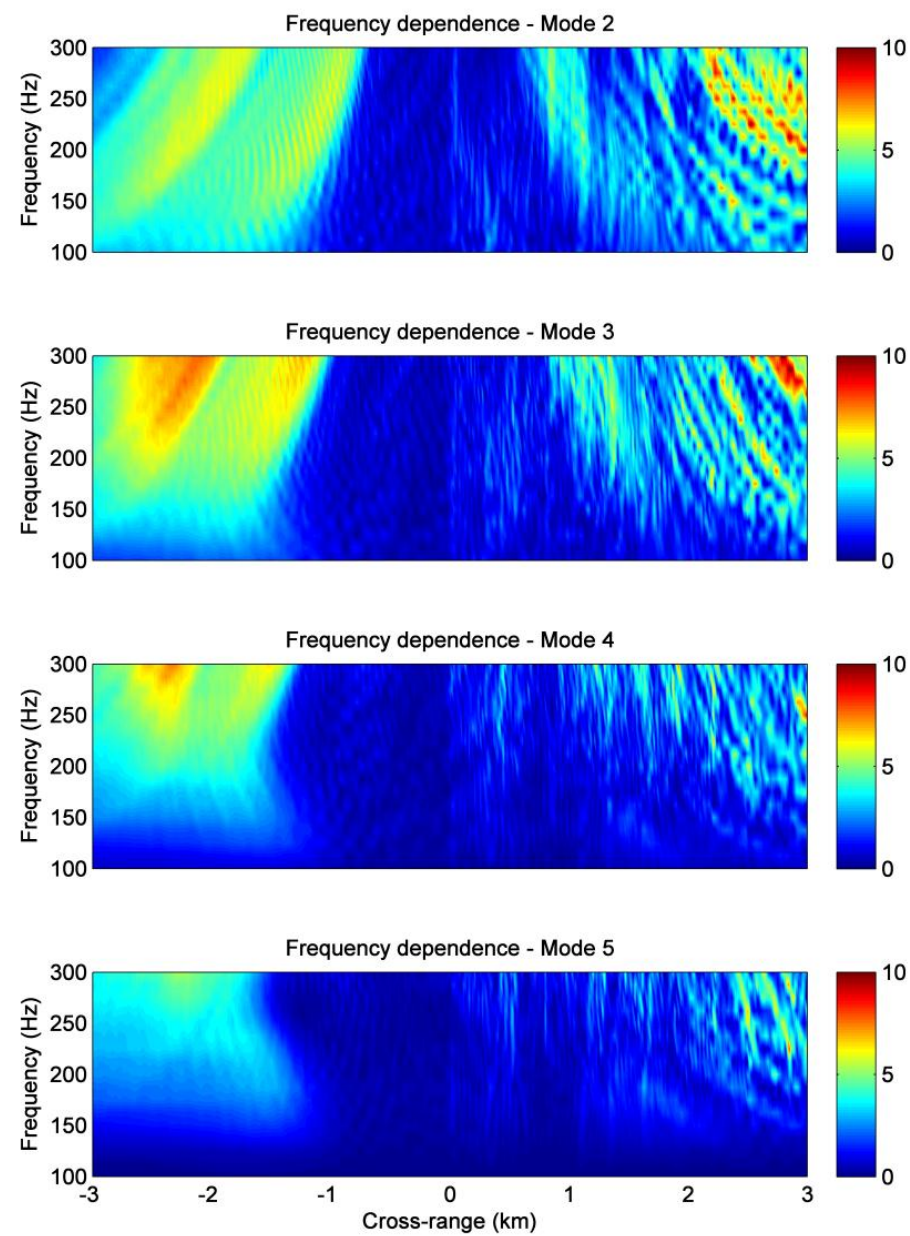

Figure 3.15 Broadband modal energy during a defocus condition is compared to modal decomposition of the PE field. Modal decomposition plots show mode amplitude at $15 \mathrm{~km}$ versus cross-range and frequency. (color online) 

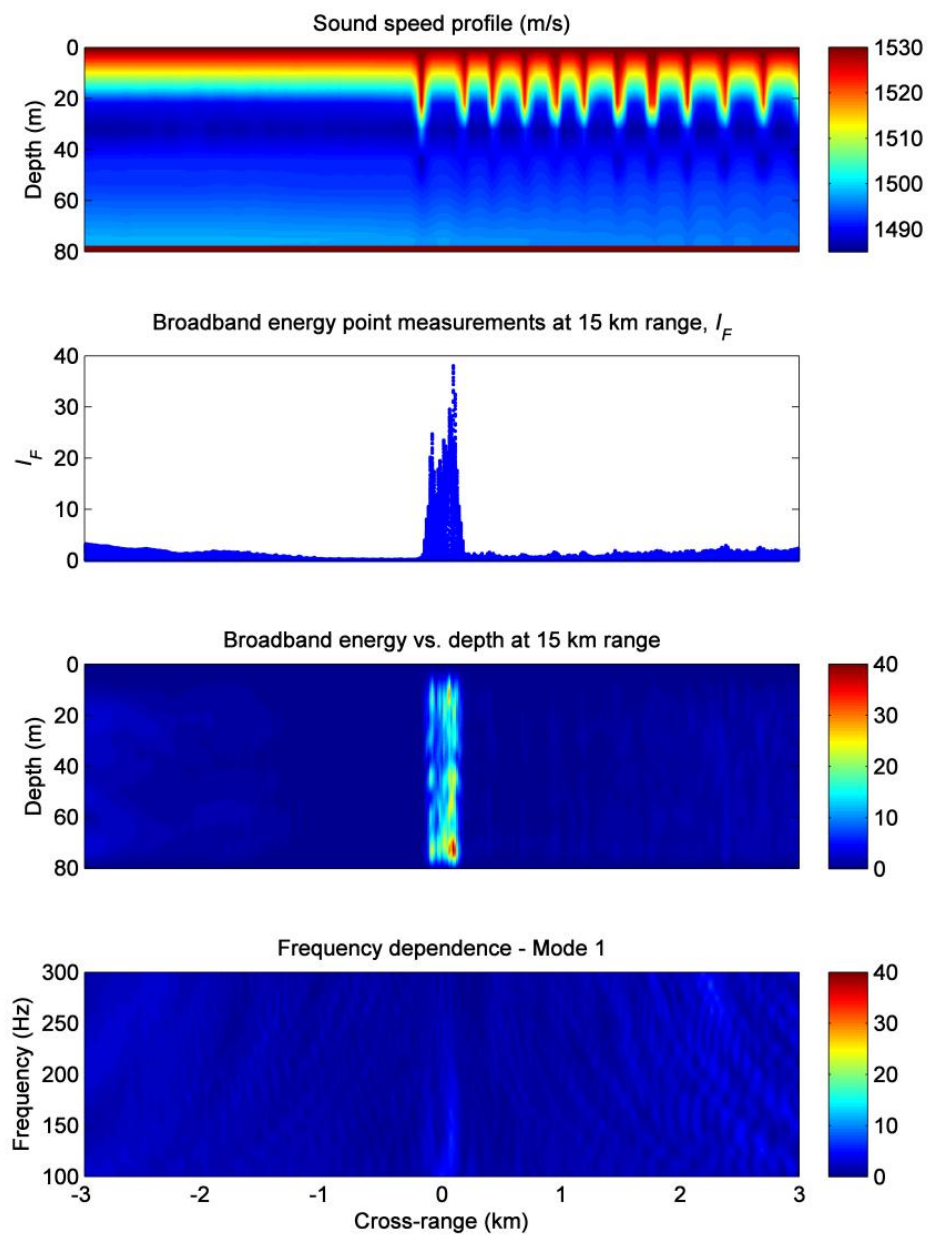

Figure 3.16 Broadband modal energy during a focus condition is compared to modal decomposition of the PE field. Modal decomposition plots show mode amplitude at $15 \mathrm{~km}$ versus cross-range and frequency. (color online) 


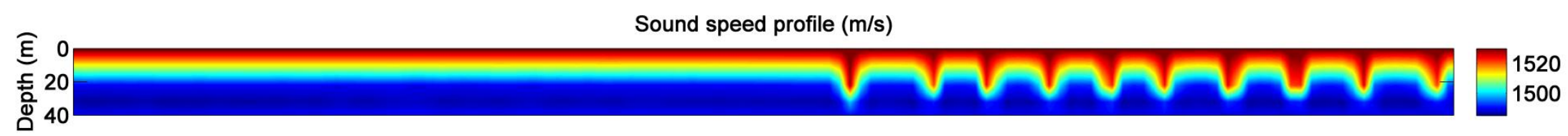

Broadband energy point measurements at $15 \mathrm{~km}$ range, $I_{F}$

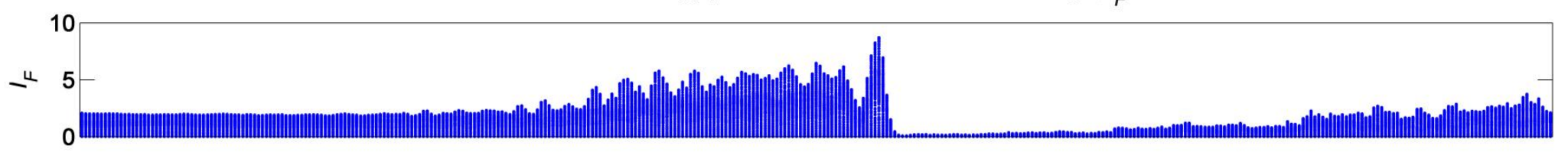

Broadband energy vs. depth at $15 \mathrm{~km}$ range
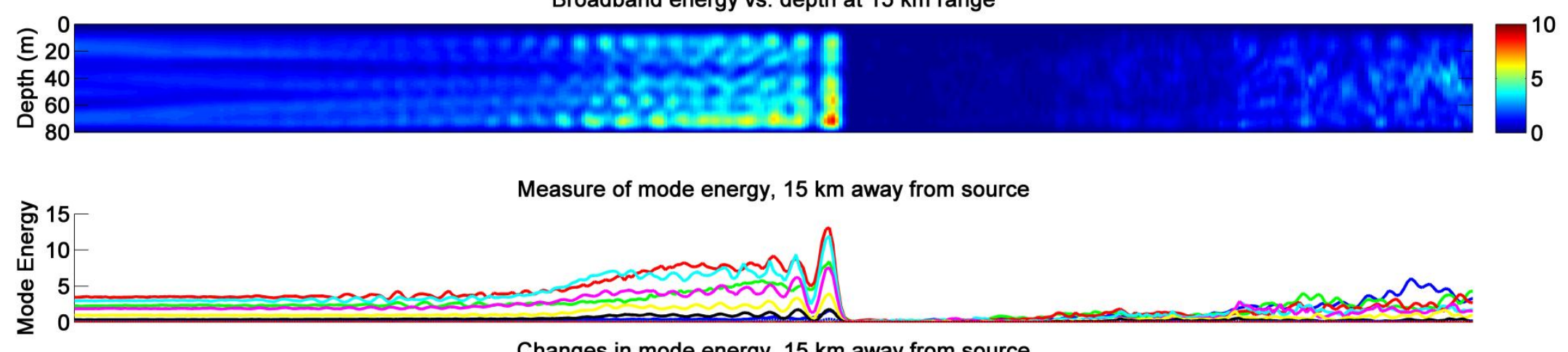

Changes in mode energy, $15 \mathrm{~km}$ away from source

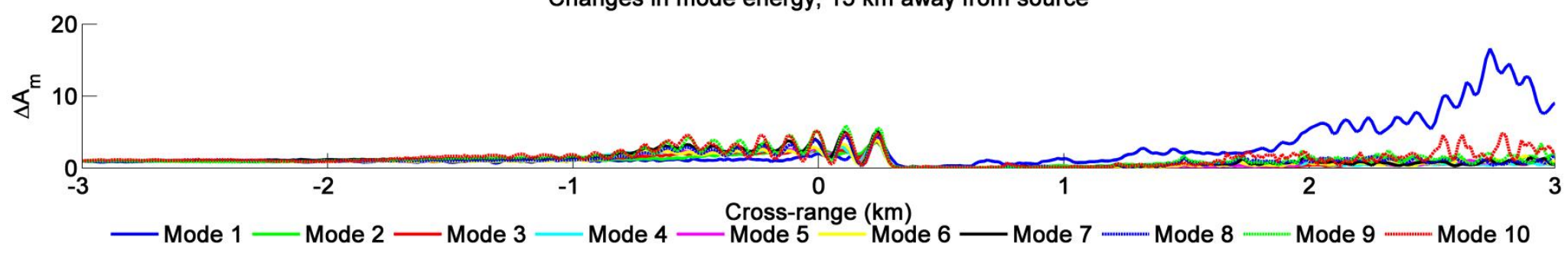

Figure 3.17 Energy distribution for a refraction scenario. Broadband energy measurements are shown in the center panels (as point measurements and versus depth). Modal energy distribution is shown on the bottom. (color online) 


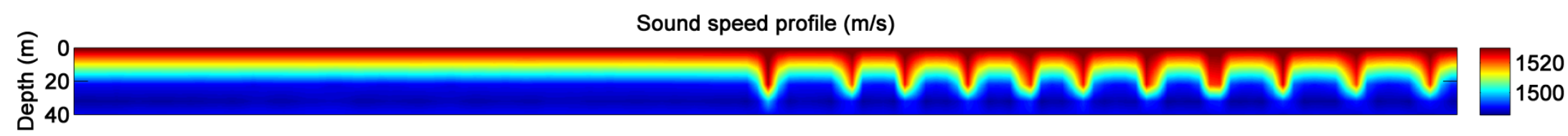

Broadband energy point measurements at $15 \mathrm{~km}$ range, $I_{F}$

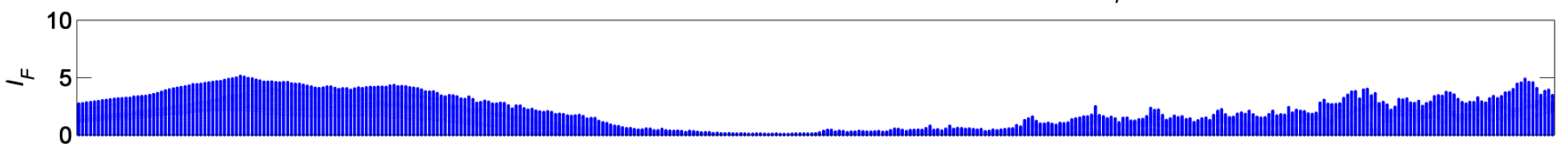

Broadband energy vs. depth at $15 \mathrm{~km}$ range

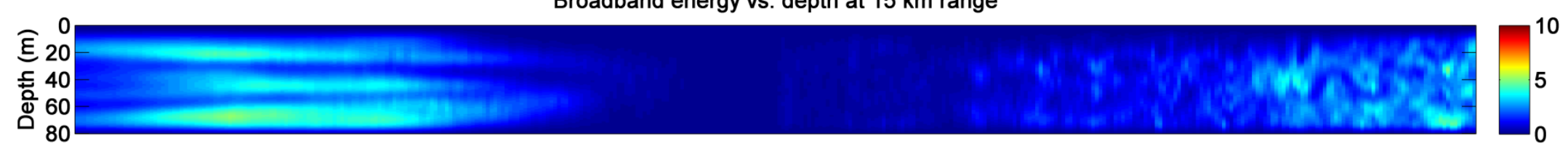

Measure of mode energy, $15 \mathrm{~km}$ away from source

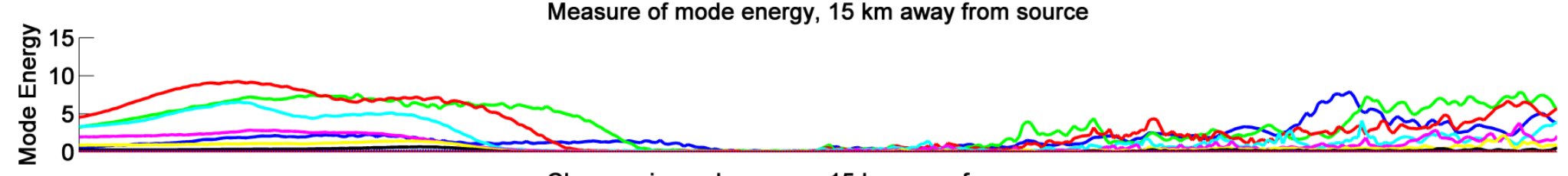

Changes in mode energy, $15 \mathrm{~km}$ away from source

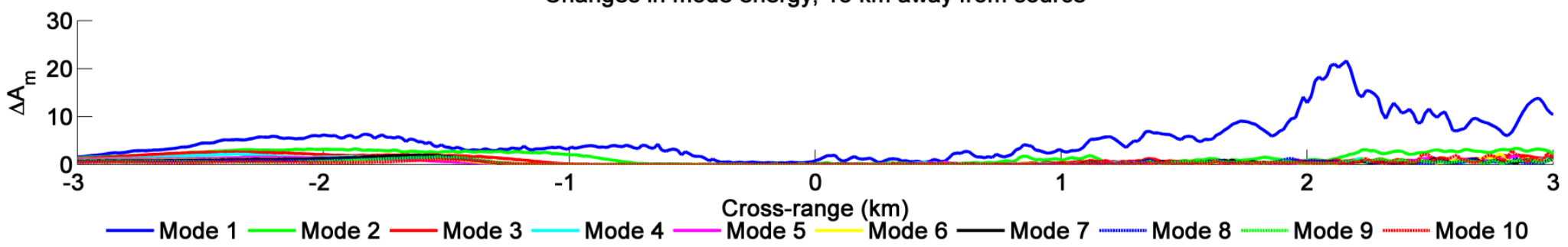

Figure 3.18 Energy distribution for a defocusing scenario. Broadband energy measurements are shown in the center panels (as point measurements and versus depth). Modal energy distribution is shown on the bottom. (color online) 


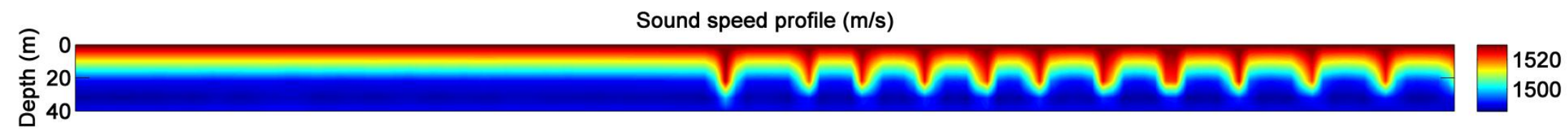

Broadband energy point measurements at $15 \mathrm{~km}$ range, $I_{F}$

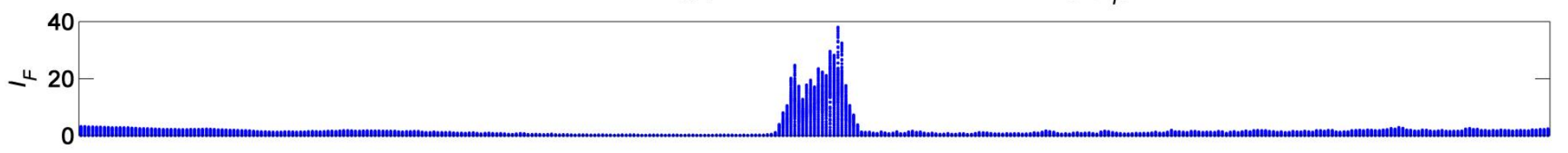

Broadband energy vs. depth at $15 \mathrm{~km}$ range
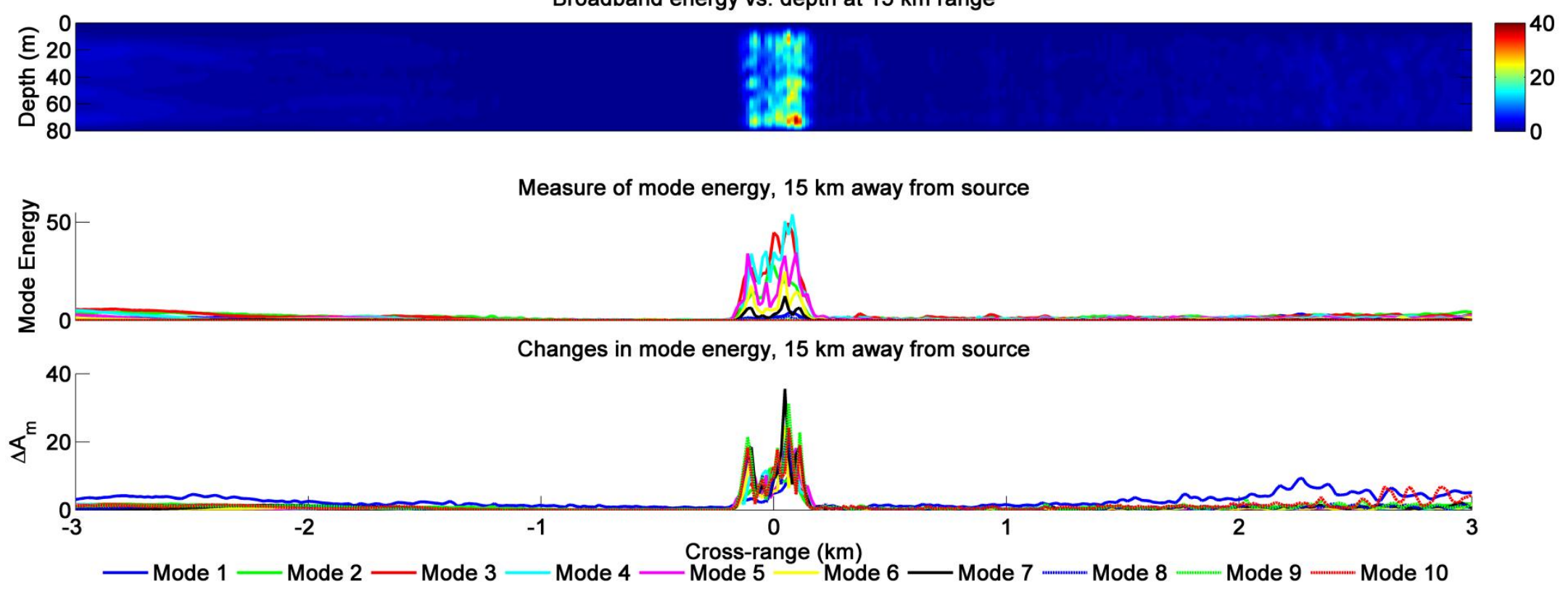

Figure 3.19 Energy distribution for a focusing scenario. Broadband energy measurements are shown in the center panels (as point measurements and versus depth). Modal energy distribution is shown on the bottom. (color online) 

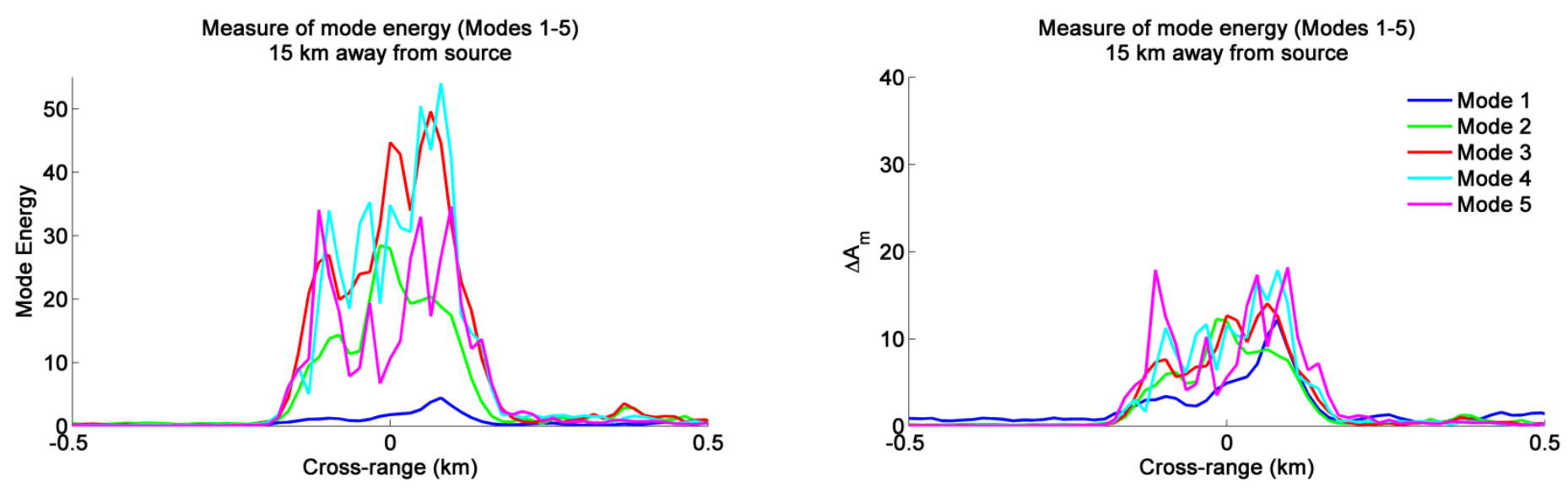

Measure of mode energy (Modes 1-5)

$15 \mathrm{~km}$ away from source

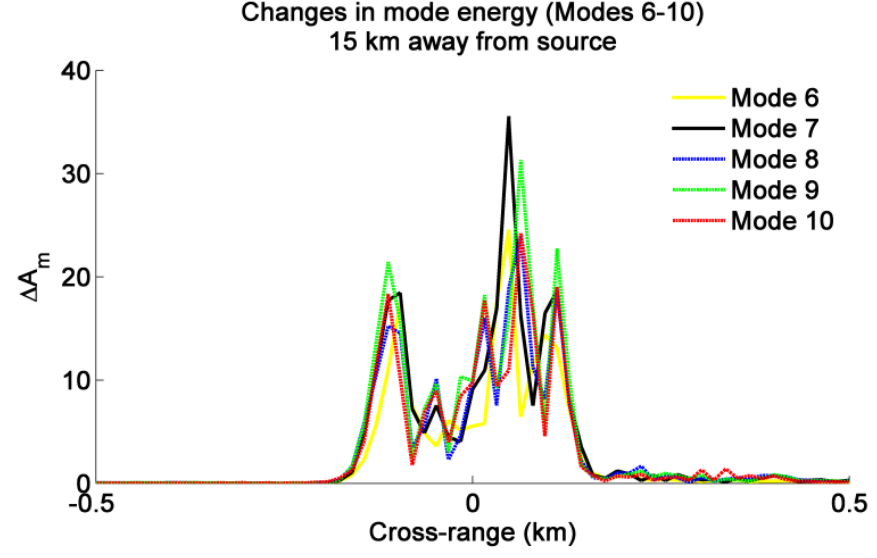

Figure 3.20 Close up of mode energy and changes in mode energy during the focus condition shown in Figure 3.19 


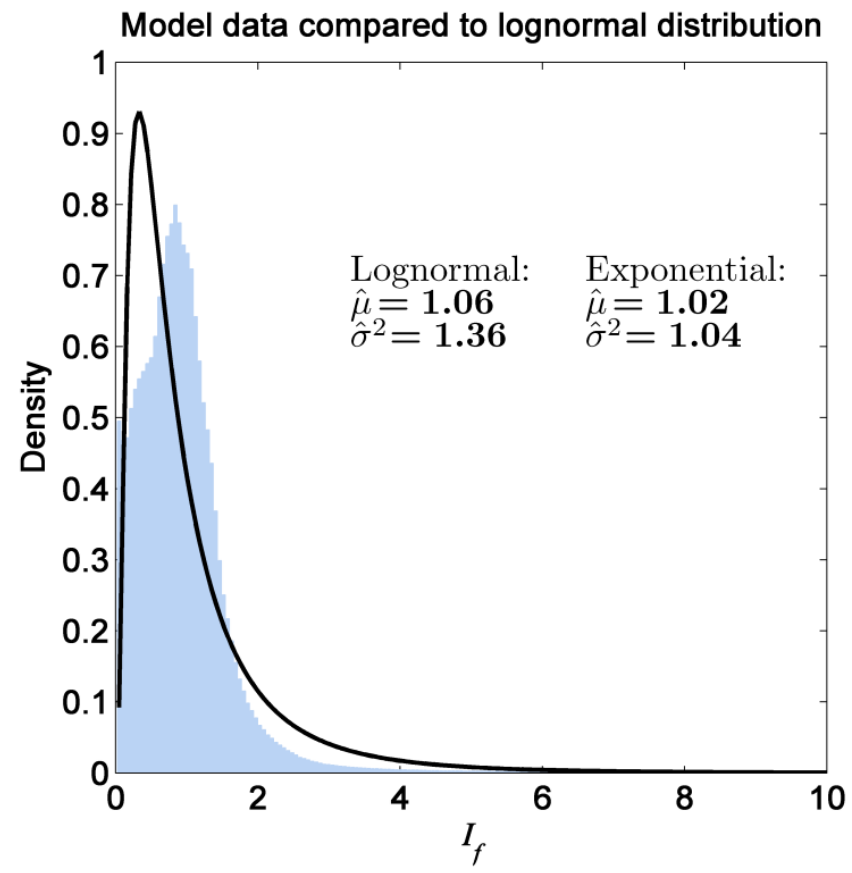

Figure 3.21 Distribution of energy for repeated PE model runs which simulated a NIW traversing the sound field. Actual model data shown by the histogram. These data are best fit to a lognormal distribution. (color online) 
Normalized "point" observations, $15 \mathrm{~km}$ away from source $4^{\circ}$ off-axis

15
$-\quad 10$
$-\quad 5$

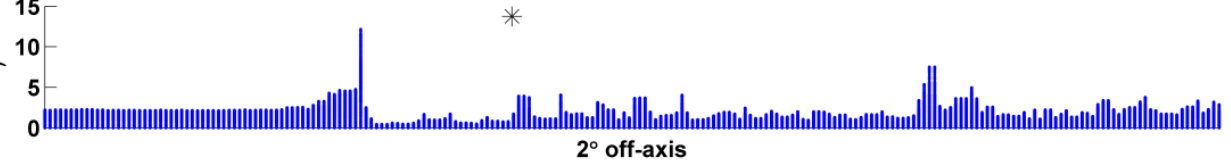

15
$-\quad 10$
$-\quad 5$

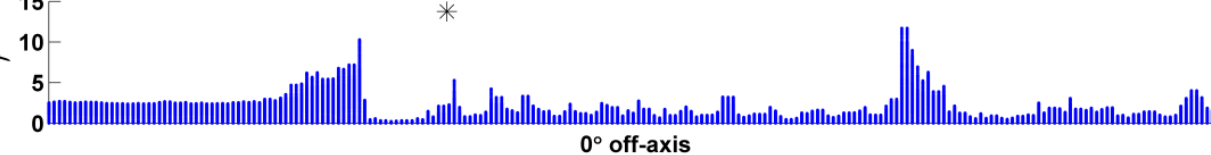

15

15
$-\quad 10$
5

$10-$
5
0

15
-10
$-\quad 5$
5

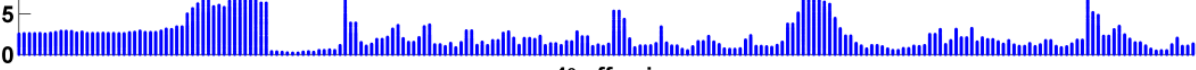

Corresponding data subset (relative to all model data)
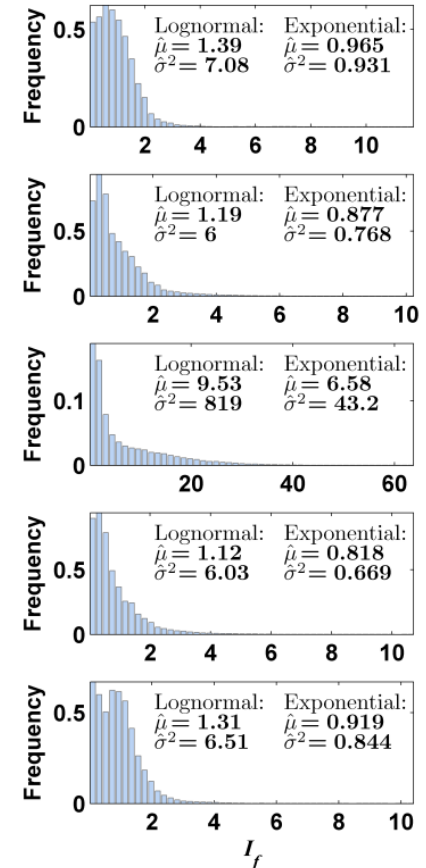

14901500151015201530

Figure 3.22 Energy dependence for instances where the acoustic track is nearly parallel to the NIW front. Acoustic angle is varied between $\pm 4^{\circ}$ to show large variability of acoustic receptions for very small angle variations. Left-most panels simulate the NIW marching across distance (or time). These data re normalized for each individual plot. Right-most panels show the associated distributions with the expected mean and variance annotated. Mean and variance values are referenced to the entire model data set. NIW sound speed at source provided as a reference. First soliton arrival at each receiving location marked by the asterisks. (color online) 

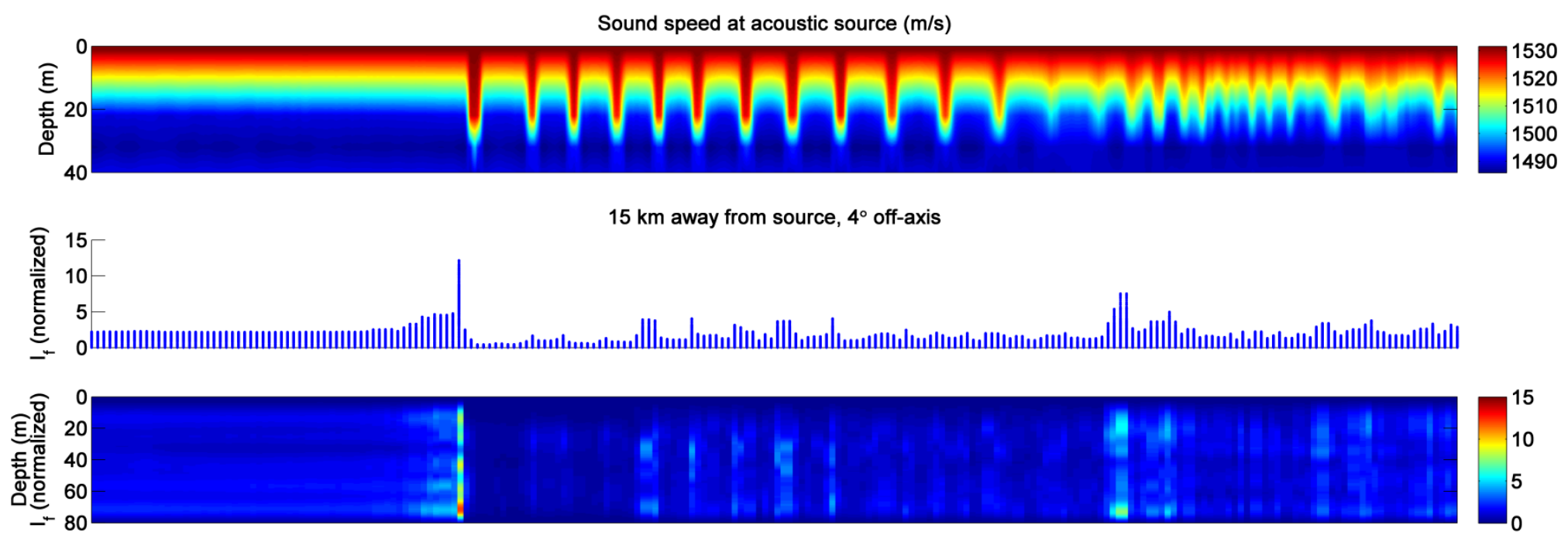

Mode energy, $15 \mathrm{~km}$ away from source, $4^{\circ}$ off-axis

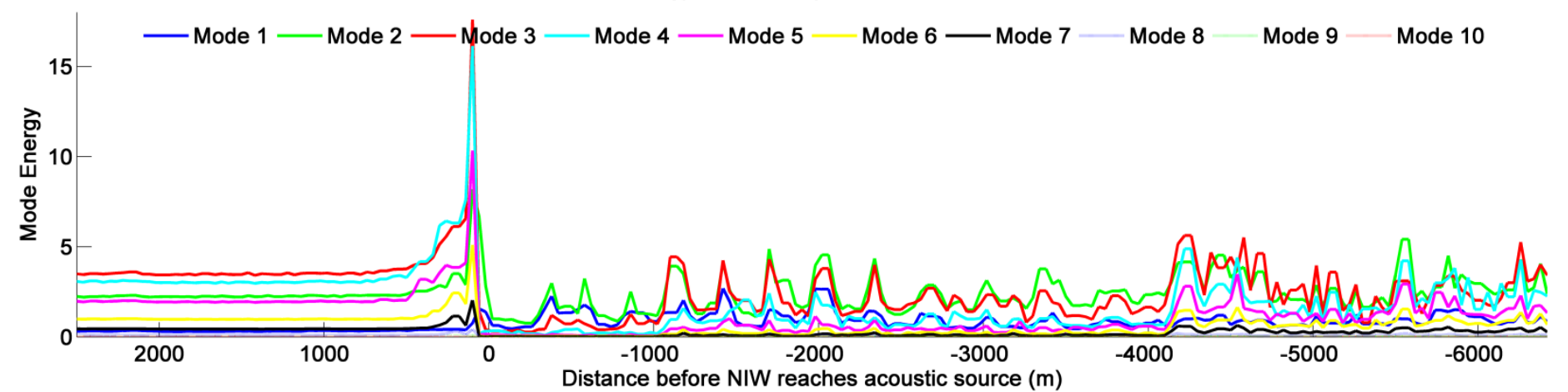

Figure 3.23 Modal energy dependence when the acoustic track is $4^{\circ}$ off-axis relative to the NIW marching across the sound field (bottom). $I_{f}$ plotted as "point" observations and versus depth to show how modal contributions influence the overall PE field (center panels). (color online) 


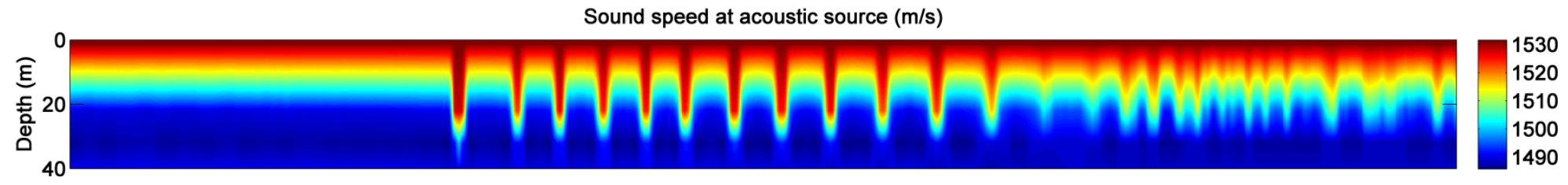

$15 \mathrm{~km}$ away from source, $2^{\circ}$ off-axis
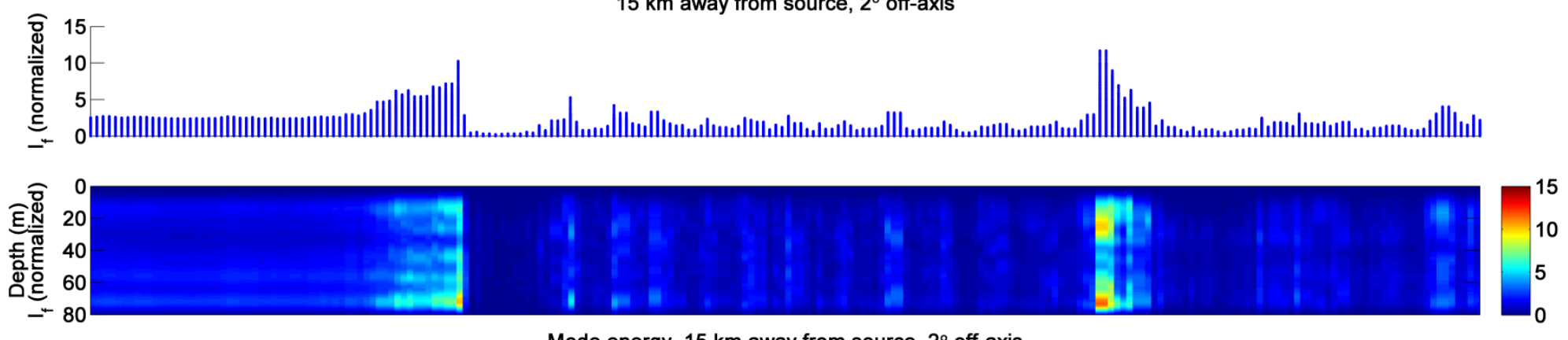

Mode energy, $15 \mathrm{~km}$ away from source, $2^{\circ}$ off-axis

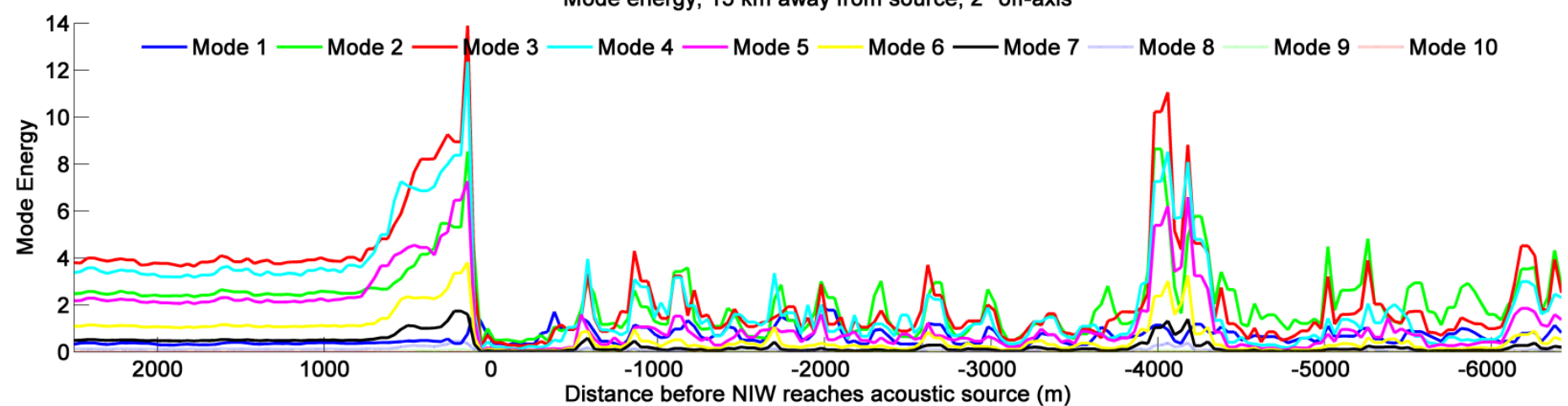

Figure 3.24 Modal energy dependence when the acoustic track is $2^{\circ}$ off-axis relative to the NIW marching across the sound field (bottom). $I_{f}$ plotted as "point" observations and versus depth to show how modal contributions influence the overall PE field (center panels). (color online) 


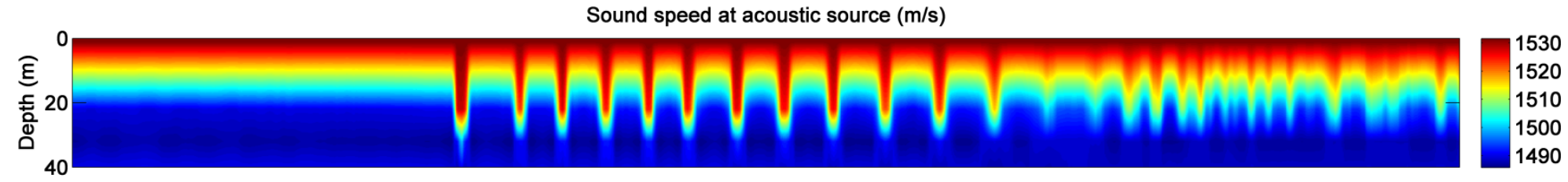

$15 \mathrm{~km}$ away from source, $0^{\circ}$ off-axis
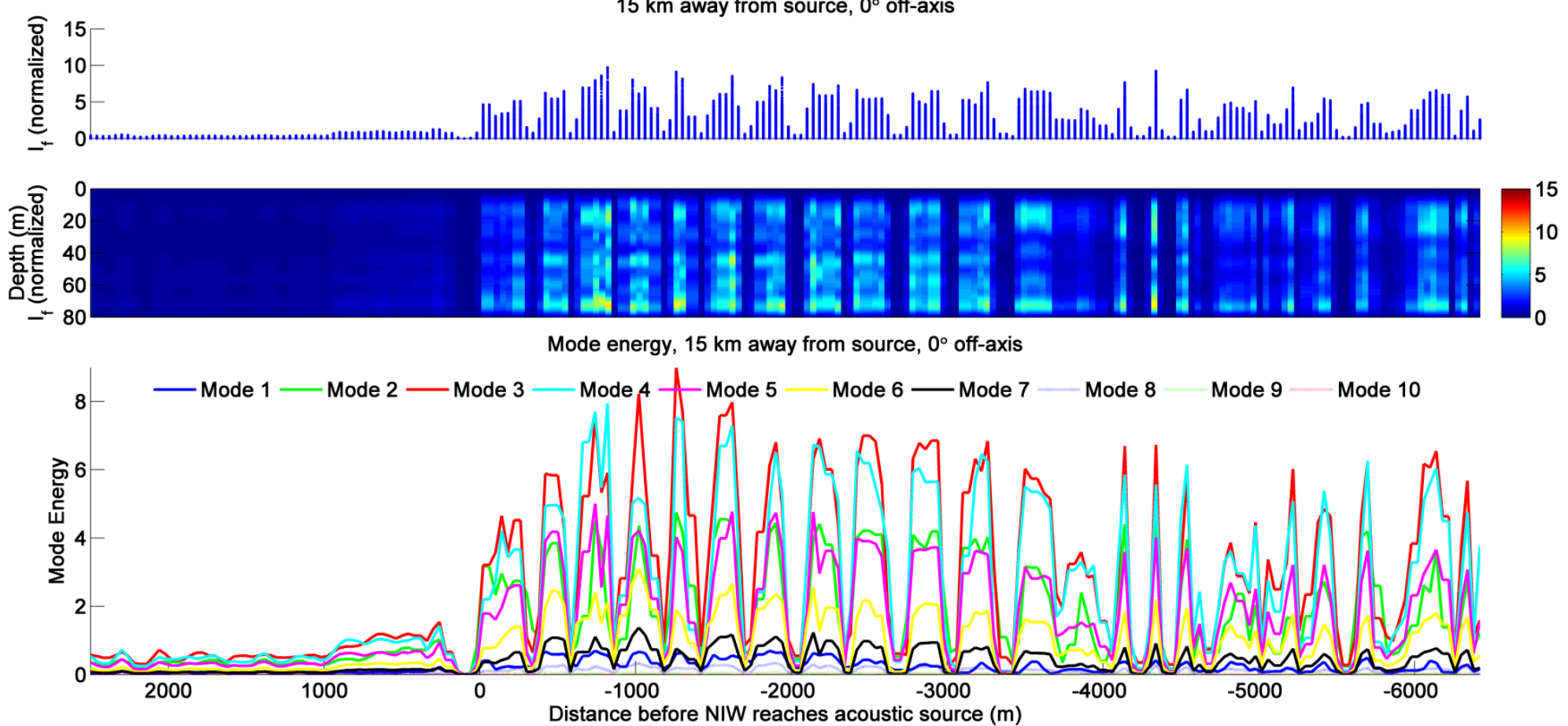

Figure 3.25 Modal energy dependence when the acoustic track is parallel to the NIW marching across the sound field (bottom). $I_{f}$ plotted as "point" observations and versus depth to show how modal contributions influence the overall PE field (center panels). (color online) 


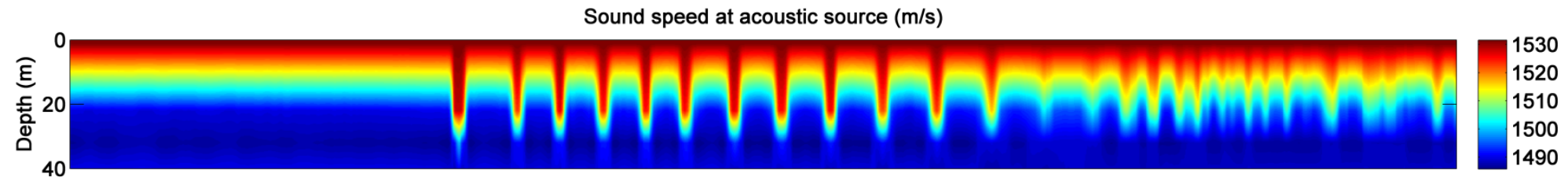

$15 \mathrm{~km}$ away from source, $-2^{\circ}$ off-axis
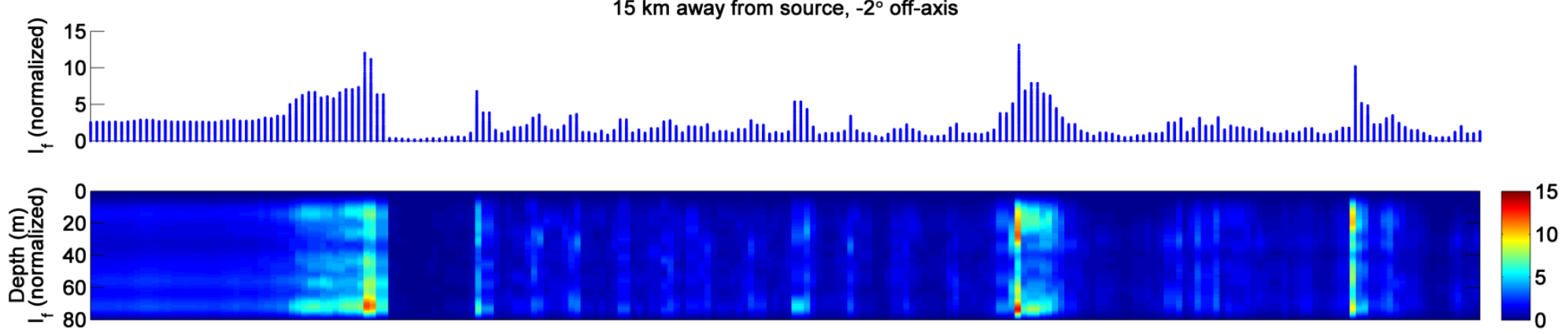

Mode energy, $15 \mathrm{~km}$ away from source, $-2^{\circ}$ off-axis

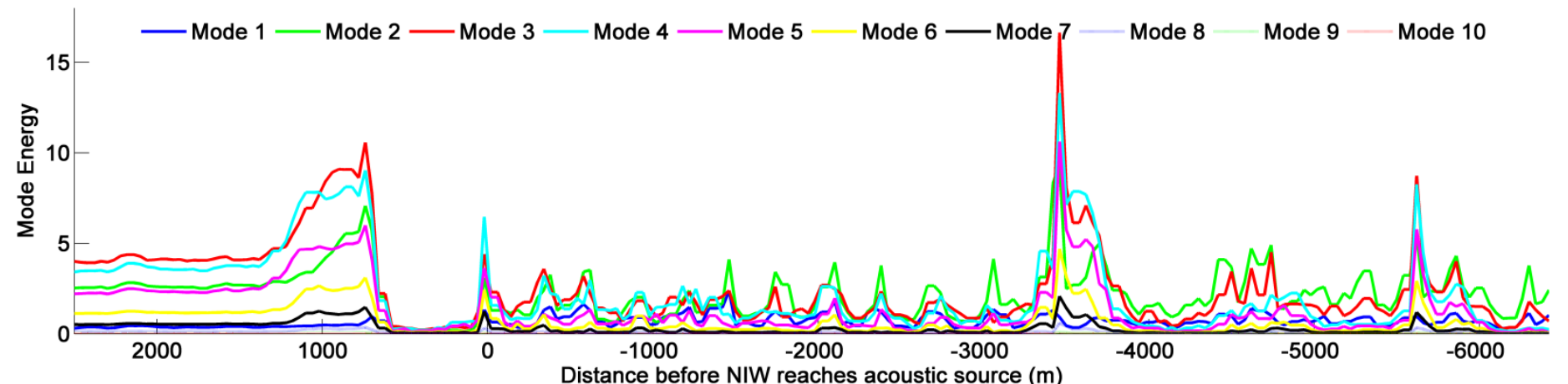

Figure 3.26 Modal energy dependence when the acoustic track is $-2^{\circ}$ off-axis relative to the NIW marching across the sound field (bottom). $I_{f}$ plotted as "point" observations and versus depth to show how modal contributions influence the overall PE field (center panels). (color online) 

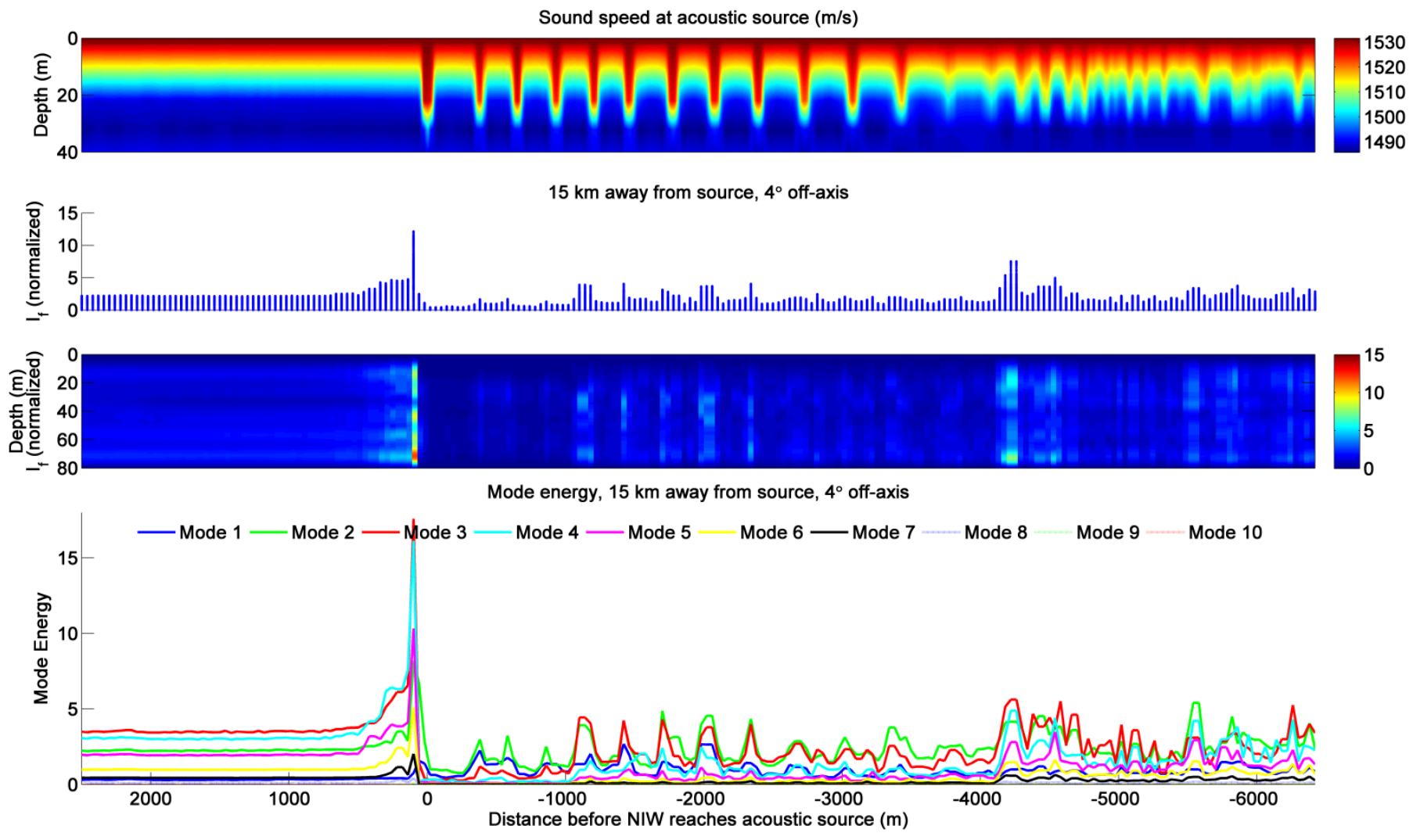

Figure 3.27 Modal energy dependence when the acoustic track is $-4^{\circ}$ off-axis relative to the NIW marching across the sound field (bottom). $I_{f}$ plotted as "point" observations and versus depth to show how modal contributions influence the overall PE field (center panels). (color online) 
Scintillation Index
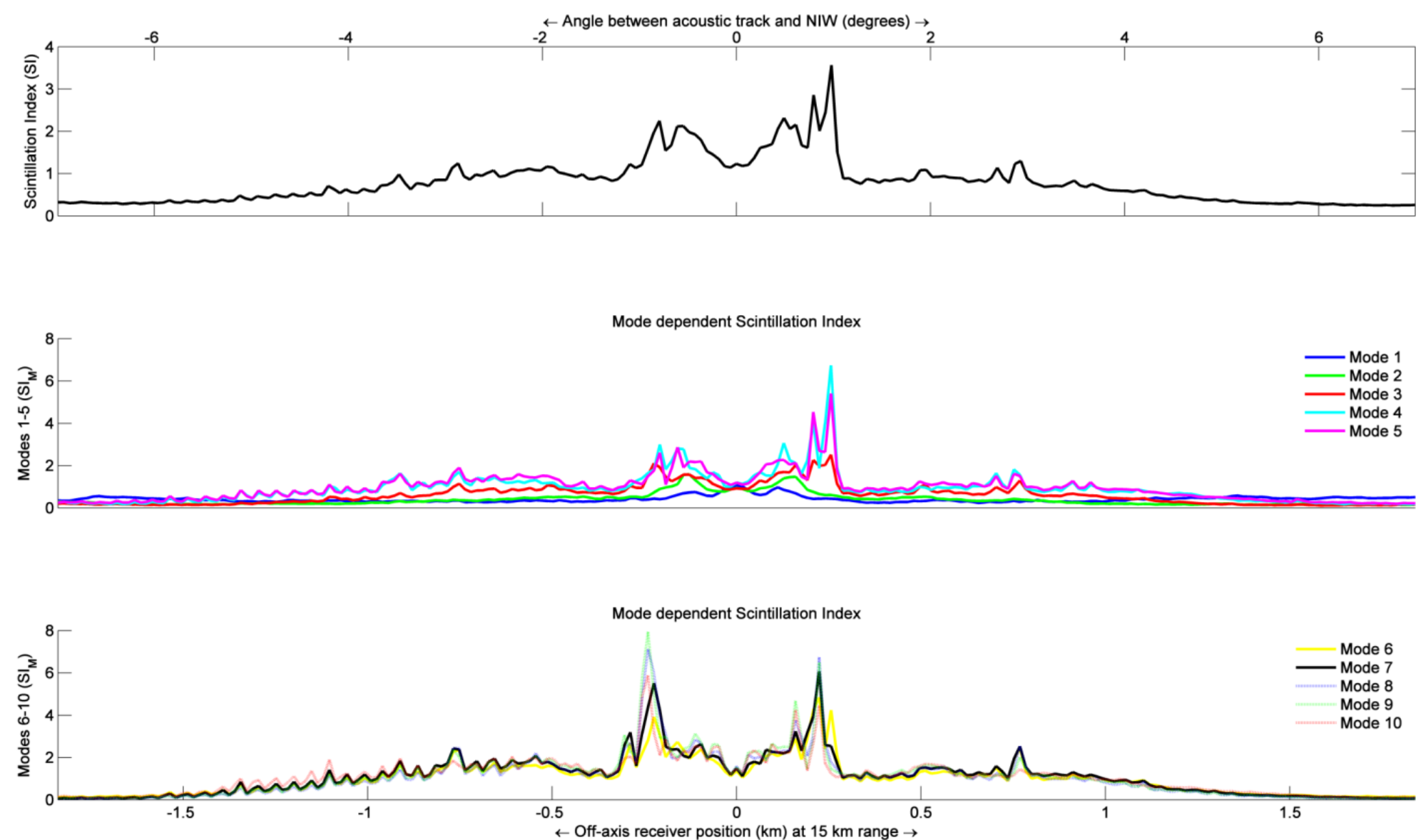

Figure 3.28 Scintillation index of broadband energy (top) and modal energy (center \& bottom) compared to receiver position relative to the acoustic source (measured in degrees or $\mathbf{k m}$ ). (color online) 

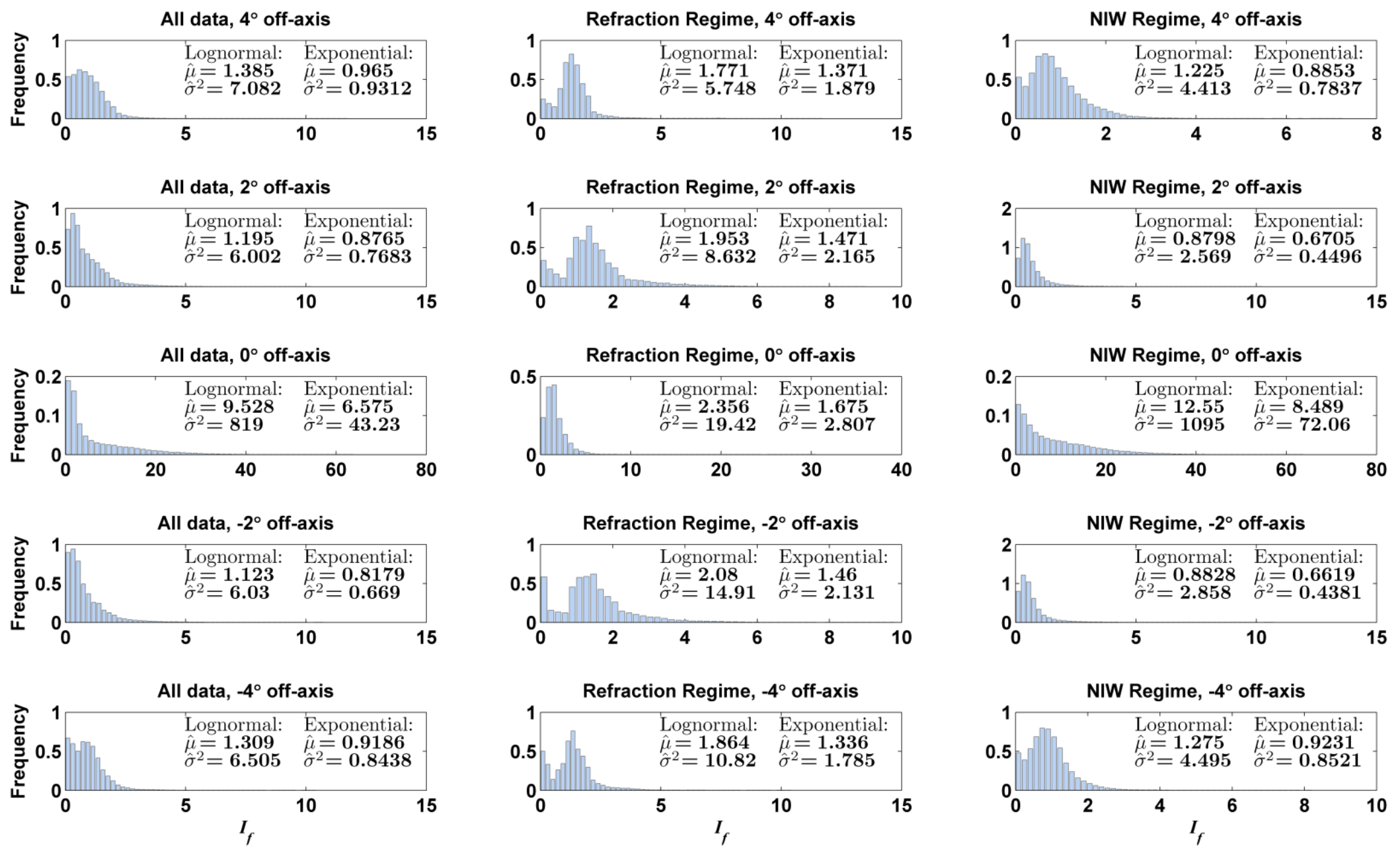

Figure 3.29 Simulated time arrival data for each bearing angle can be further divided into time-varying fluctuation regimes. Histograms and statistical mean and variance parameters help quantify the differences between dominant physical processes. 


\section{APPENDIX A. MEASURED DATA CONSIDERATIONS \& ADDITIONAL NIW EVENTS}

\section{A.1 Event 44 Noise Analysis.}

An interesting phenomenon appeared coincident with Event 44, which was the presence of increasing ambient noise as the NIW approached the acoustic track. Changes in the ambient noise field due to (or in the presence of) NIWs has been addressed by work in previous studies. ${ }^{1-3}$ As NIWs are known to modulate manmade signals, they are also known to influence the ambient noise field. Our concern with Event 44 data is the possibility that noise encroaches upon our signal of interest, corrupting the data. The primary argument for this possibility is that refraction prior to the NIW's arrival should be on the order of minutes, rather than hours, which is suggested by the measured data.

In order to examine this issue, a "back of the envelope" calculation was performed, which replicated Event 44 in its simplest terms. Using measured data from environmental mooring SW32, we searched for a reasonable critical via Snell's law by (1) differences in sound speed, and (2) differences in modal eigenvalues from equation (2.12). Figure A.1 shows the interpolated sound speed profile from environmental mooring SW32 and a single sensor trace at the displaced thermocline. A baseline sound speed was calculated by averaging the two hour timeframe prior to the NIW's arrival. The bottom panel shows a critical angle estimate in the horizontal plane based upon temperature perturbations at this sensor trace. This method yields a maximum critical angle of roughly $13^{\circ}$. Similarly, Figure A. 2 shows mode and frequency dependent critical angles based upon KRAKEN modeling. Here, the maximum value is roughly $8^{\circ}-$ a relatively high value for NIW activity when compared to an expected critical angle on the order of $4-5^{\circ}$. 
A straight-line geometry for Event 44 was assumed, creating the simple geometrical problem shown in Figure A.3. Based upon the NIW's arrival between the source and receiver, and also from radar imagery, we believe the angle difference between the acoustic track and the NIW front to be $5-6^{\circ}$. This yields a maximum start-time for horizontal refraction around 30-35 minutes before the NIW reaches the acoustic track, or receiving VLA. Because this is shorter than what the measured data indicates, we investigate the potential for noise corrupting the data, and conducted a secondary analysis of the measured data shown throughout Manuscript I and II.

For our secondary analysis, our goal was to mitigate noise. Differences between our original approach in calculating $I_{\tau}$ versus this secondary method are as follows:

- The original data shown in Manuscript I and II was based upon the complex demodulates, and also was high-pass filtered at $f_{c}=100 \mathrm{~Hz}$.

- The data in the secondary analysis has been band-pass filtered between $f_{b p}=100-300 \mathrm{~Hz}$ as this was the quietest spectrum of these data.

- In the secondary analysis, the signal is not down-sampled in any fashion, and we calculate energy metrics using the actual time-arrival data, and not the complex envelope.

- In both instances, the signals were match filtered.

- In the secondary analysis we employ a threshold approach where any data that falls below a statistical level of certainty is ignored. 
- In the secondary analysis we keep the same "tight" limits for our time window $\tau$ when integrating under the energetic region of the signal

Figure A.4 shows example measured data during an instance of low ambient noise for hydrophones at the top, middle, and bottom of the VLA. This chirp sequence was recorded at the very beginning of the Event 44 dataset, at the start of (or prior) to the ramping phenomenon. Figure A.6 shows a similar plot for a period of high ambient noise, these data recorded as the first soliton reaches the VLA. In both instances, the left panels represent the entire chirp sequence, and the right panels a close-up of the first chirp arrival. The grey signal represents the pressure time sequence received upon each phone after the band-pass and match filtering has taken place. The black overlay represents the portion of the received signal that falls within the integration window used to calculate received energy $I_{\tau}$-these limits remained the same for both methods. The red portion represents noise before and after each chirp, and the light blue line represents an $85 \%$ noise threshold based upon a statistical analysis of the noise.

Figure A.5 and Figure A.7 show a statistical analysis of noise received for both chirp sequences, originally shown in Figure A.4 and Figure A.6. The values presented here represent received pressure after the filtering process. The top panel shows a statistical boxplot of noise received upon each hydrophones of the VLA. From this, one infers that noise is depth-dependent, and a single threshold level cannot be used across the entire array depth. As an example of one hydrophone, the bottom-left panel shows a histogram of noise on the eighth hydrophone, and a fitted Gaussian PDF. The noise is normally distributed with zero mean. The bottom right-panel shows the corresponding CDF with vertical grey lines representing 75, 85, 90, 95, and 99 percent confidence levels. From 
these confidence levels, we can create meaningful intensity thresholds that are tailored to each individual chirp arrival based upon arrival time and phone depth. In our secondary calculation of $I_{\tau}$, signals within the integration window that fall below the chosen threshold level are ignored, with the assumption that this would represent ambient noise contributions between modal arrivals. We note that the noise appears to be loudest towards the bottom of the VLA, and that the example for high noise has an increased variance when compared to the low noise example.

Figure A.8 shows recalculated $I_{\tau}$ metrics using a $90 \%$ threshold and Figure A.9 using an $85 \%$ threshold. In both cases the ramping feature exists longer than the anticipated 30-35 minutes, and the general trend compared to our original calculations remain the same. In our original analysis we removed any samples that were completely overshadowed by noise, and the only remaining instance where noise contributions can be a considered suspect is the potential focusing that occurs at 5:40 GMT. However, this particular subset of data includes very strong chirp arrivals, where both received signal and noise levels are comparably higher. An unfortunate consequence to our secondary method is that the ensemble data set lost the "goodness of fit" to a lognormal distribution, and began to approach a generalized extreme value distribution, indicating we were ignoring portions of each chirp arrival.

Based on this secondary analysis we retained the original $I_{\tau}$ calculations for Manuscripts I and II. Event 44 had unique features that help explain long-term refraction. The wave propagated very slowly and its strong structure created a high critical angle for refraction in the horizontal plane. Because we did not sample the water 
column at sufficient locations, we cannot explain the exact NIW evolution for this particular event, and our simple geometry shown in Figure A.3 may be deficient. The "de-noising" of these data still keeps the original ramping trend, and we are therefore confident that we measure signal fluctuations, even if the ambient noise field is also changing.

\section{A.2 Additional NIW events}

Two additional NIW events indicate probable oceanographic modulation of the acoustic field. These events were labeled Event 45 and Event 46 . Unfortunately, we do not have sufficient data to estimate the angle between the propagating NIW and the acoustic track. Since both these events occurred at location C1D, based upon the expected angle these NIWs propagated, we can safely assume horizontal refraction

effects dominated. We analyze the identical chirp signal that was previously described.

Event 45 occurred at 18:00 GMT on 14 August and lasted roughly two hours. Figure A.11 shows point observations versus the ADCP echo at the acoustic source, and versus sound speed at the receiving array. Environmental measurements indicate NIW activity existed throughout the entire acoustic track, and energy metrics show a fluctuating sound field. Figure A.12 shows the statistical breakdown of these data, which again are well-fit by a lognormal PDF. Two acoustic ducts appear in the water column at roughly 20 and 50 meters.

Event 46 occurred at 16:30 GMT on 16 August 2006, also at location C1D. Figure A.13 shows $I_{\tau}$ versus oceanographic measurements at the source and receiver. In this instance NIW activity is lighter. The ADCP echo shows NIW activity that may be 
considered a Mode 2 internal wave. The acoustic data shows possible refraction prior to the NIW's arrival. Figure A.14 shows the statistics of these data, which are also well approximated by a lognormal distribution. In this instance, energy appears to dominate at the bottom of the water column.

\section{REFERENCES AND LINKS}

1. D. Rouseff and D. Tang, "Internal wave effects on the ambient noise notch in the East China Sea: Model/data comparison," The Journal of the Acoustical Society of America 120 (3), 1284-1294 (2006).

2. D. Rubenstein, "Observations of cnoidal internal waves and their effect on acoustic propagation in shallow water," Oceanic Engineering, IEEE Journal of 24 (3), 346-357 (1999).

3. Q. Li, D. M. Farmer, T. F. Duda and S. Ramp, "Acoustical Measurement of Nonlinear Internal Waves Using the Inverted Echo Sounder," J. Atmos. Oceanic Technol. 26 (10), 2228-2242 (2009). 


\section{FIGURES}
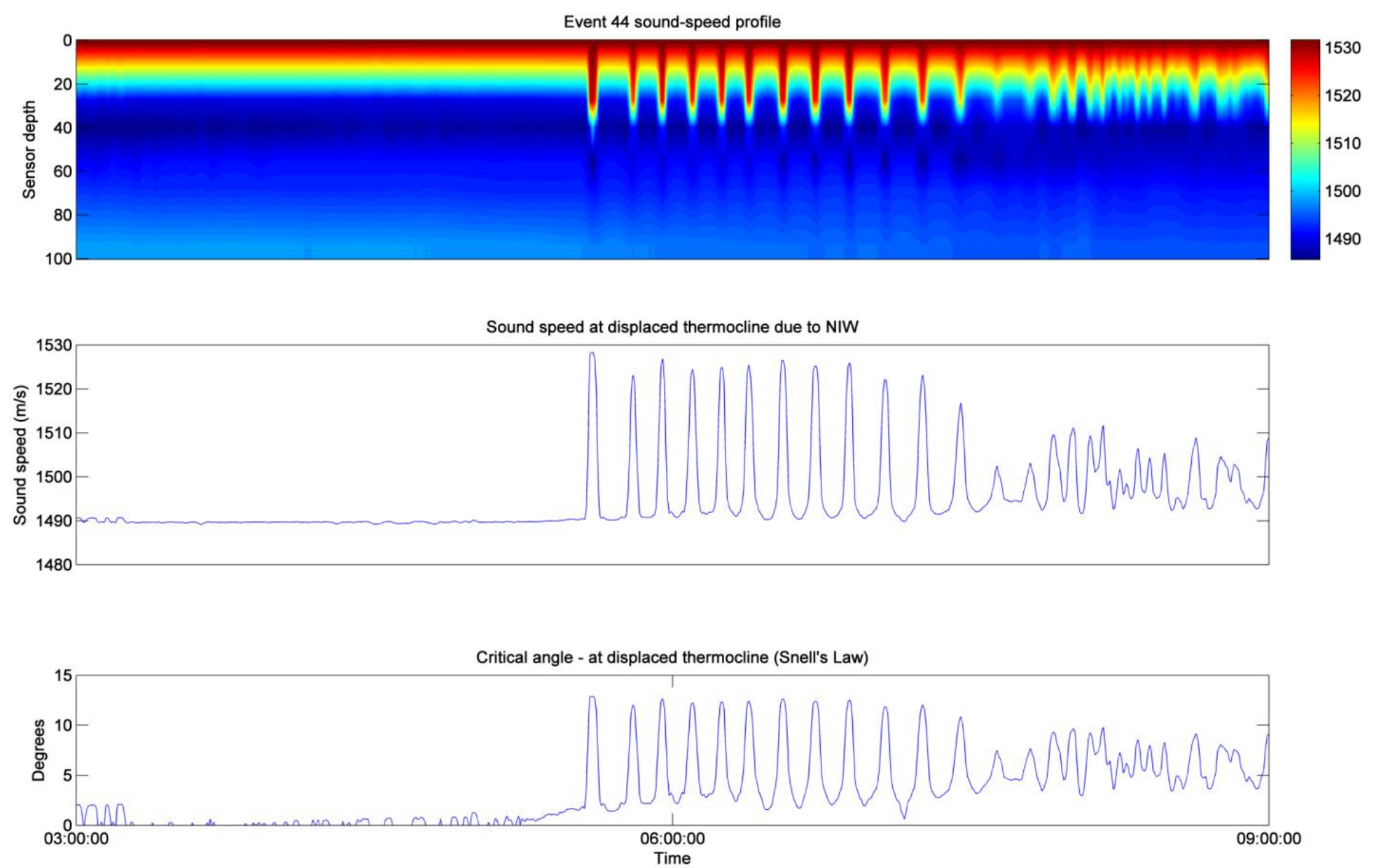

Figure A.1 Event 44 sound speed profile (top). Sound speed at the displaced thermocline (middle). Critical angle, using Snell's law, and measurements from the displaced thermocline (bottom). 

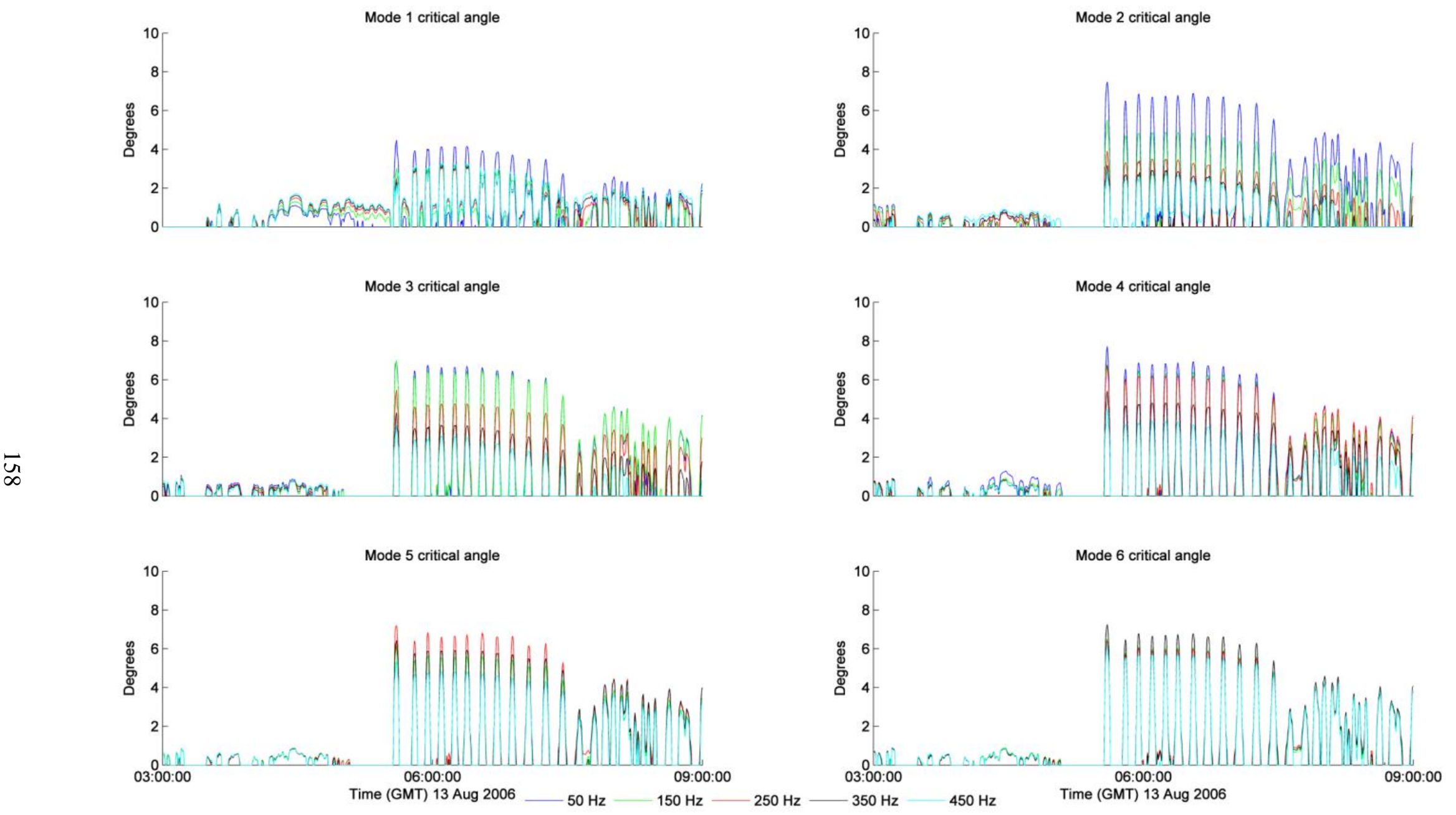

Figure A.2 Mode and frequency dependent critical angles for Event 44 . 


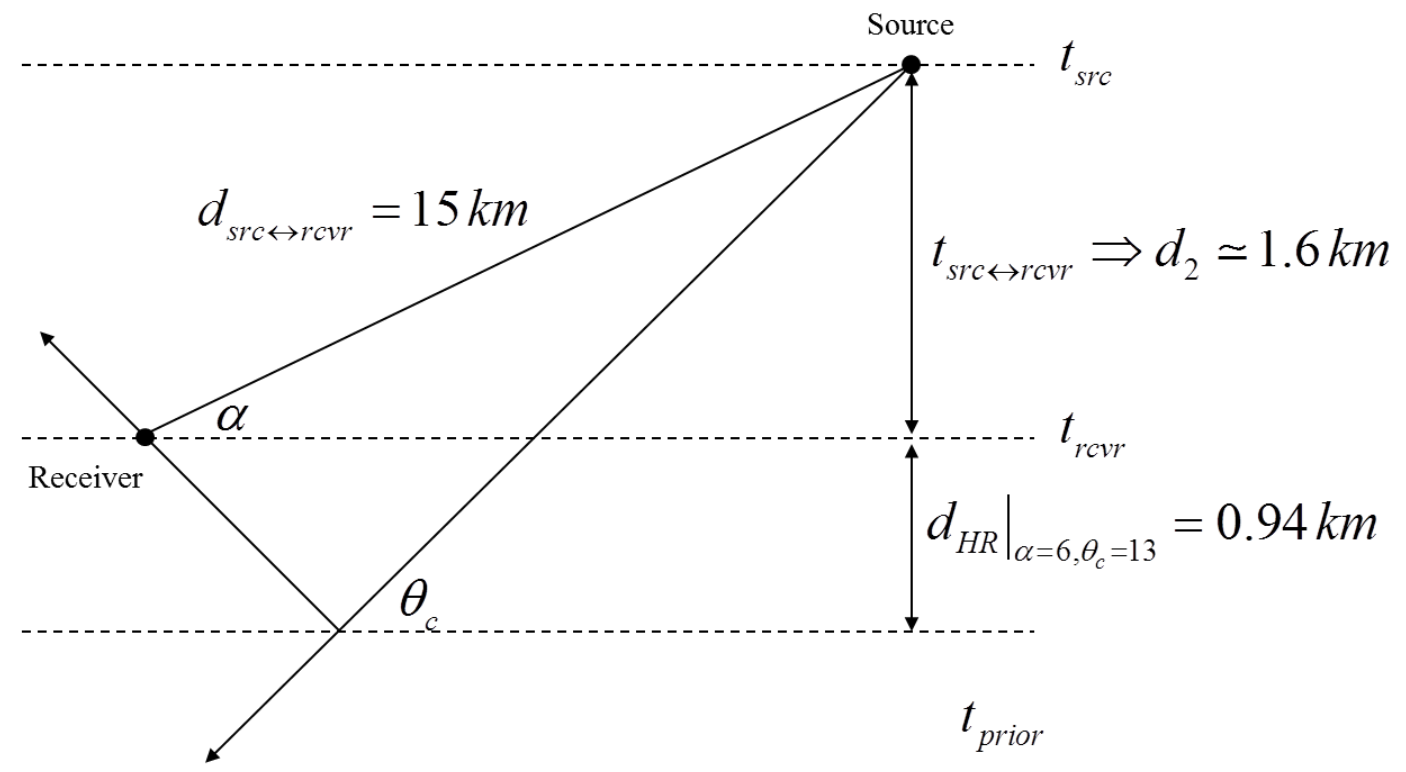

Approaching NIW Front $\uparrow$

$$
\begin{aligned}
& d_{H R}= d_{s r c \leftrightarrow r c v r}\left[\frac{\cos \alpha \sin \theta_{c}-\sin \alpha \cos \theta_{c}}{2 \cos \theta_{c}}\right] \\
& \therefore \max \left(t_{H R}\right) \simeq 33 \text { minutes }
\end{aligned}
$$

Figure A.3 "Back of the envelope" calculation used to estimate maximum time and distance horizontal refraction is likely to take place for Event 44 


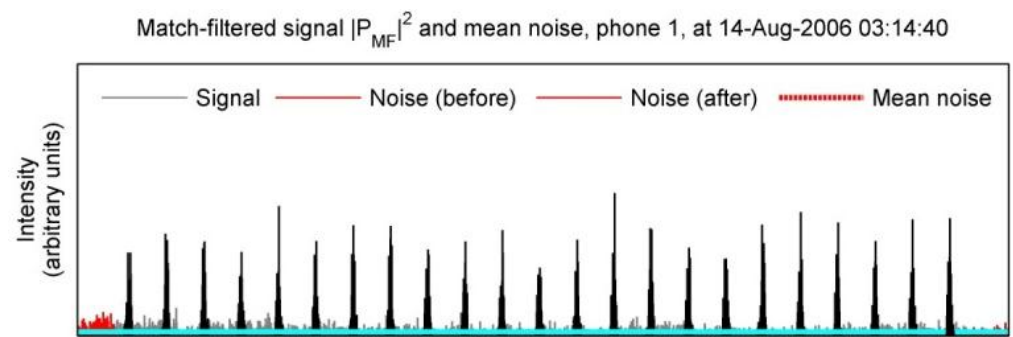

Match-filtered signal $\left|\mathrm{P}_{\mathrm{MF}}\right|^{2}$ and mean noise, phone 8, at 14-Aug-2006 03:14:40
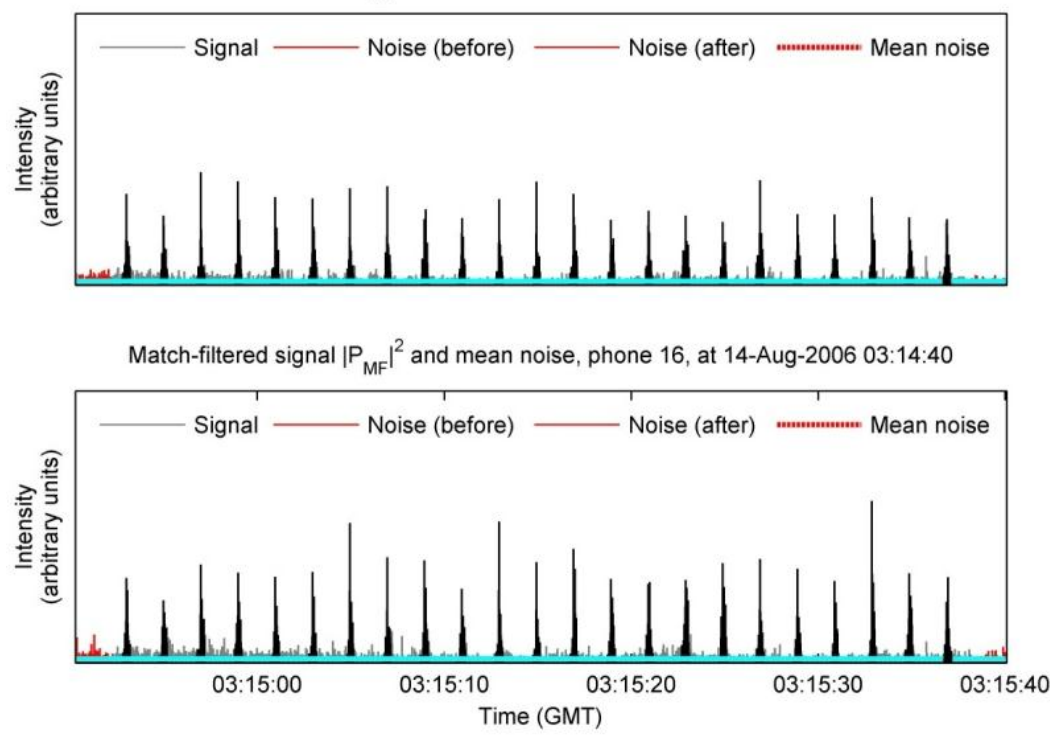

Integration window for chirp 1

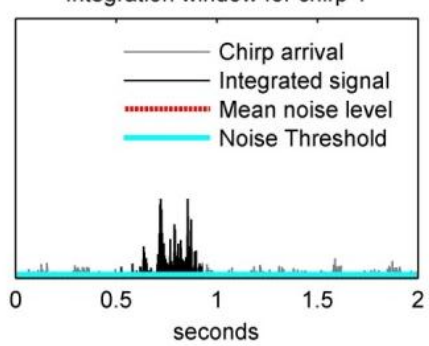

Integration window for chirp 1

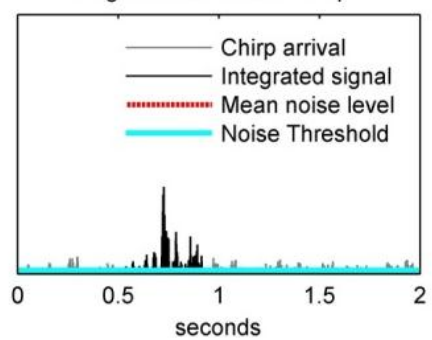

Integration window for chirp

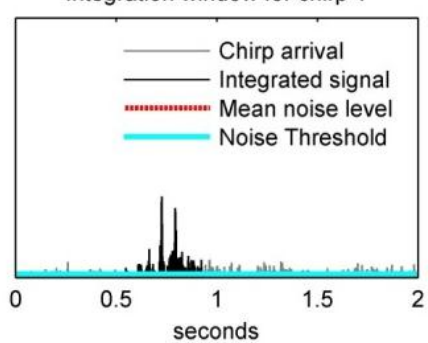

Figure A.4 Example chirp sequence for arrivals with low ambient noise levels for hydrophones at the top, middle and bottom of the VLA. This example from the start of the entire Event 44 data set. Entire measured signal shown in grey. Signal that falls within the limits of integration shown in black. Noise before and after each chirp sequence shown in red. $85 \%$ noise threshold shown as a light blue bar. First chirp arrival for each sequence expanded in the right panels. 
Noise distribution for each VLA hydrophone
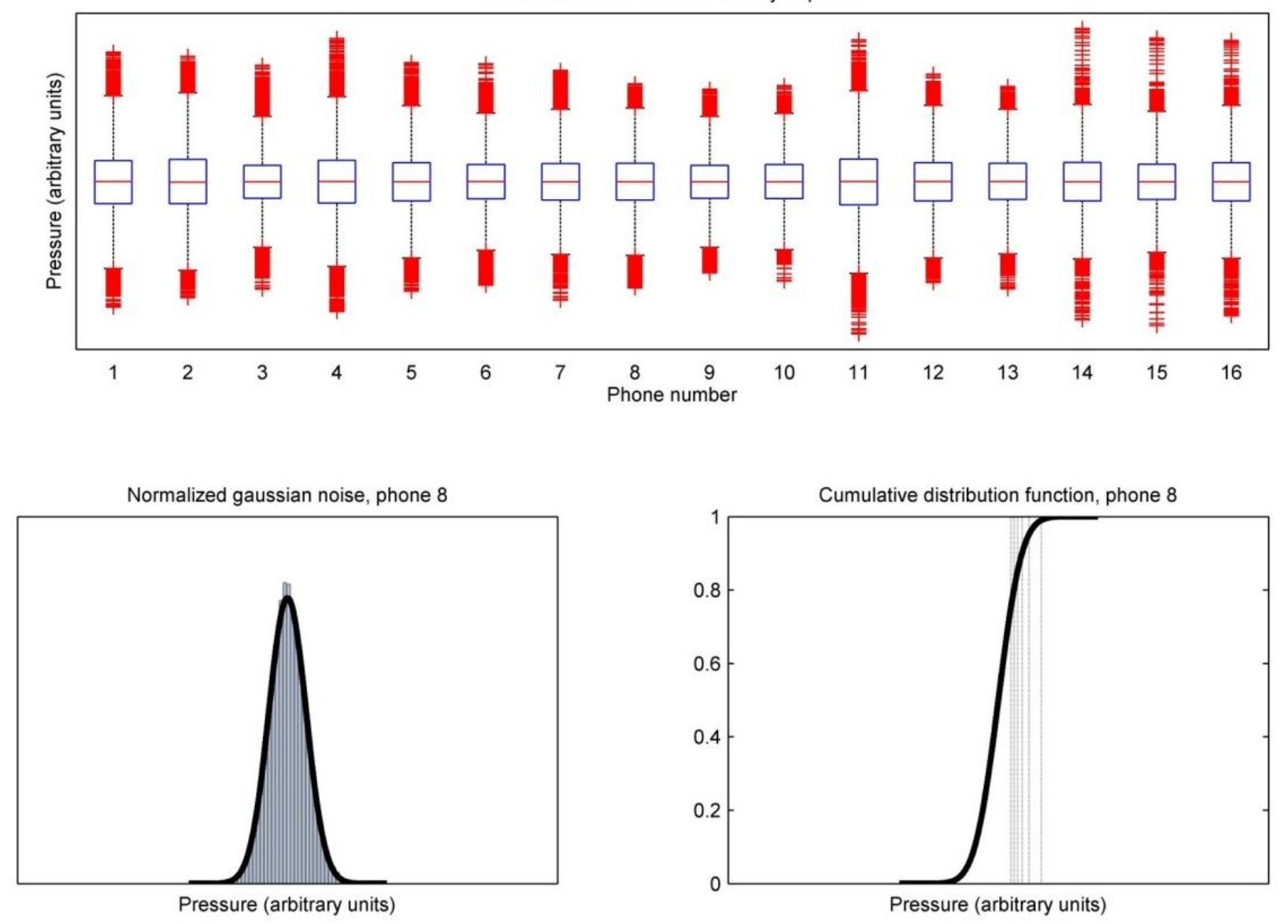

Figure A.5 Boxplot representing measured ambient at each phone before and after chirp sequence shown in Figure A.4 (top). Noise proves to be normally distributed as shown by histogram and fitted Gaussian PDF (bottom left). Associated CDF with vertical lines corresponding to $75,80,85,90,95$, and $99 \%$ probability. 


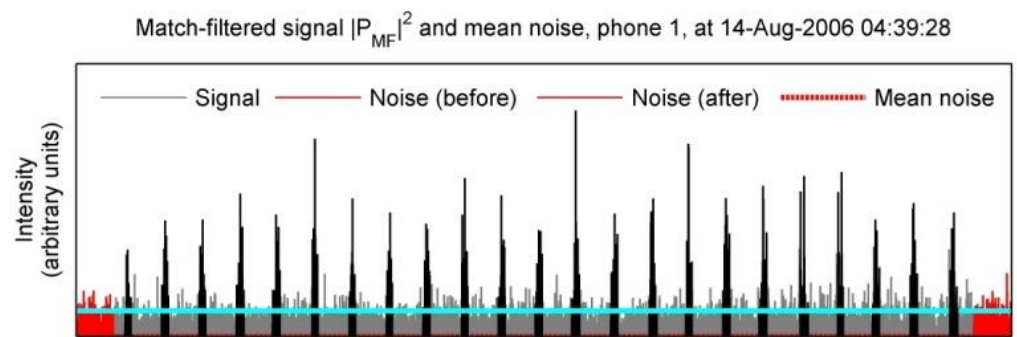

Match-filtered signal $\left|\mathrm{P}_{\mathrm{MF}}\right|^{2}$ and mean noise, phone 8, at 14-Aug-2006 04:39:28

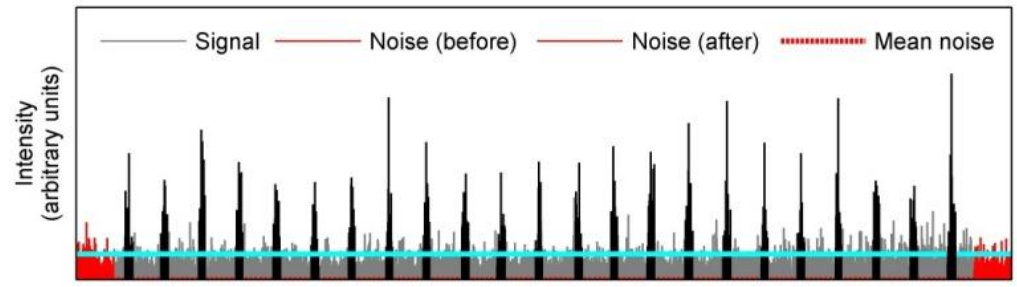

Match-filtered signal $\left|\mathrm{P}_{\mathrm{ME}}\right|^{2}$ and mean noise, phone 16, at 14-Aug-2006 04:39:28

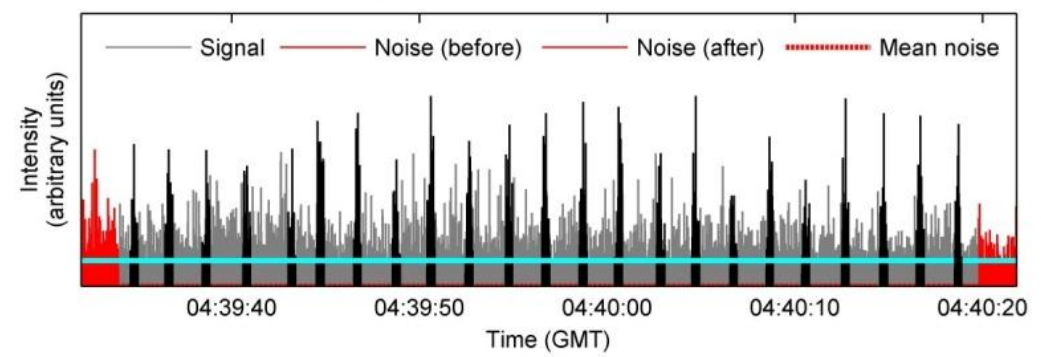

Integration window for chirp 1

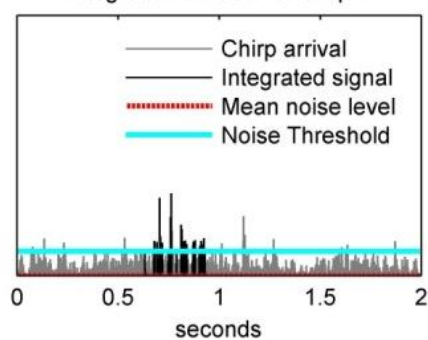

Integration window for chirp

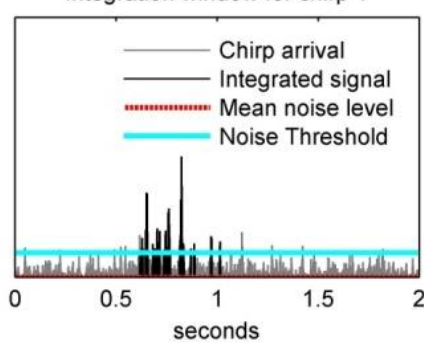

Integration window for chirp 1

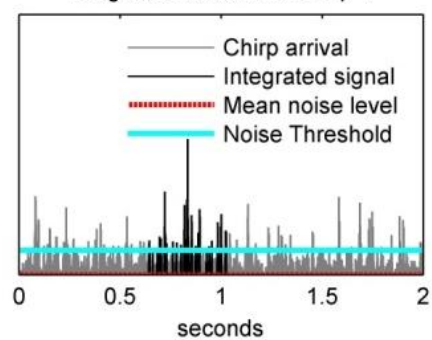

Figure A.6 Example chirp sequence for arrivals with high ambient noise levels for hydrophones at the top, middle and bottom of the VLA. This example taken as the first soliton arrives at the VLA. Entire measured signal shown in grey, vertical scale is identical to Figure A.4. Signal that falls within the limits of integration shown in black. Noise before and after each chirp sequence shown in red. $85 \%$ noise threshold shown as a light blue bar. First chirp arrival for each sequence expanded in the right panels. 

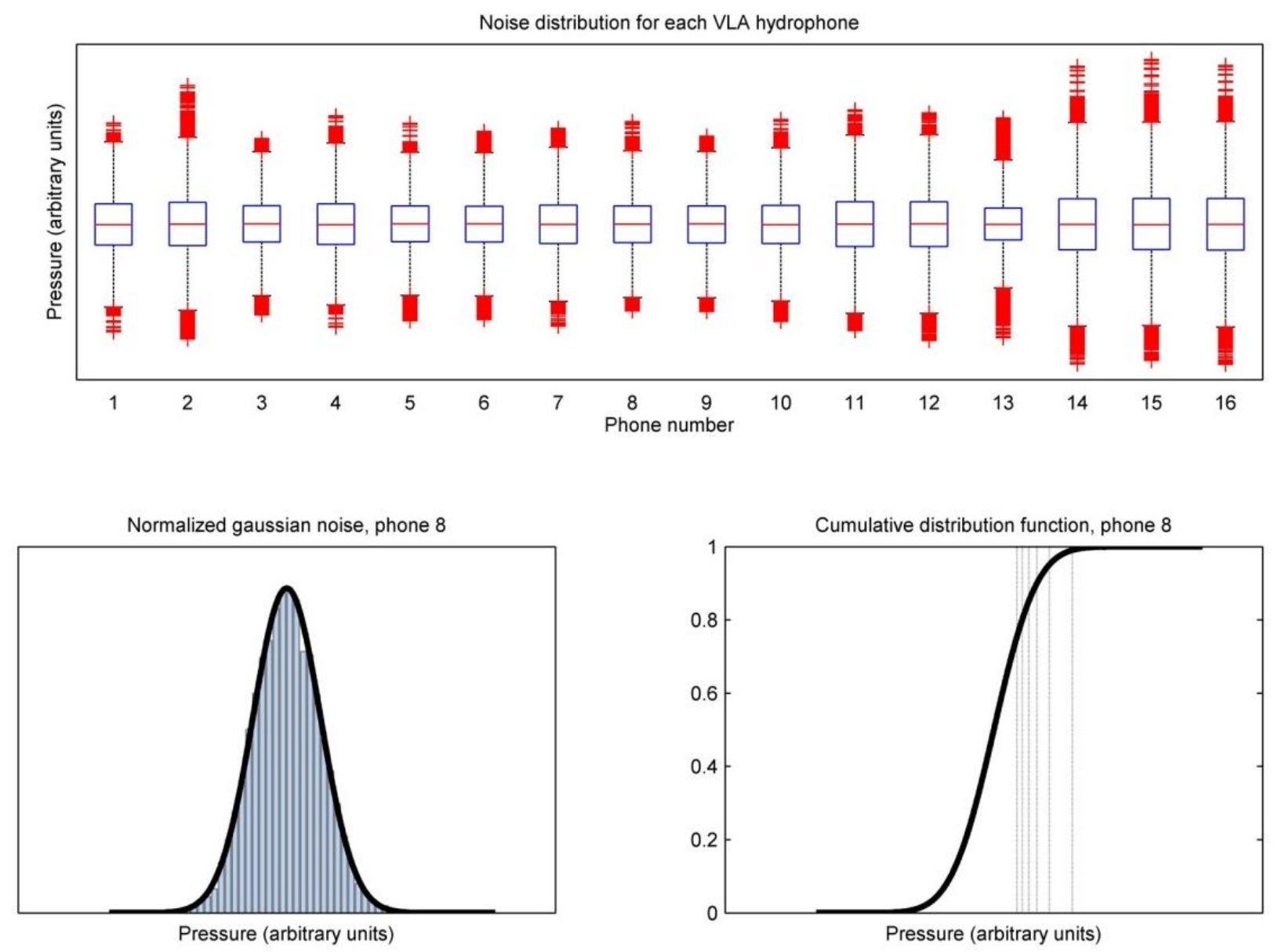

Figure A.7 Boxplot representing measured ambient at each phone before and after chirp sequence shown in Figure A.6 (top). Noise proves to be normally distributed as shown by histogram and fitted Gaussian PDF (bottom left). Associated CDF with vertical lines corresponding to $75,80,85,90,95$, and $99 \%$ probability. 

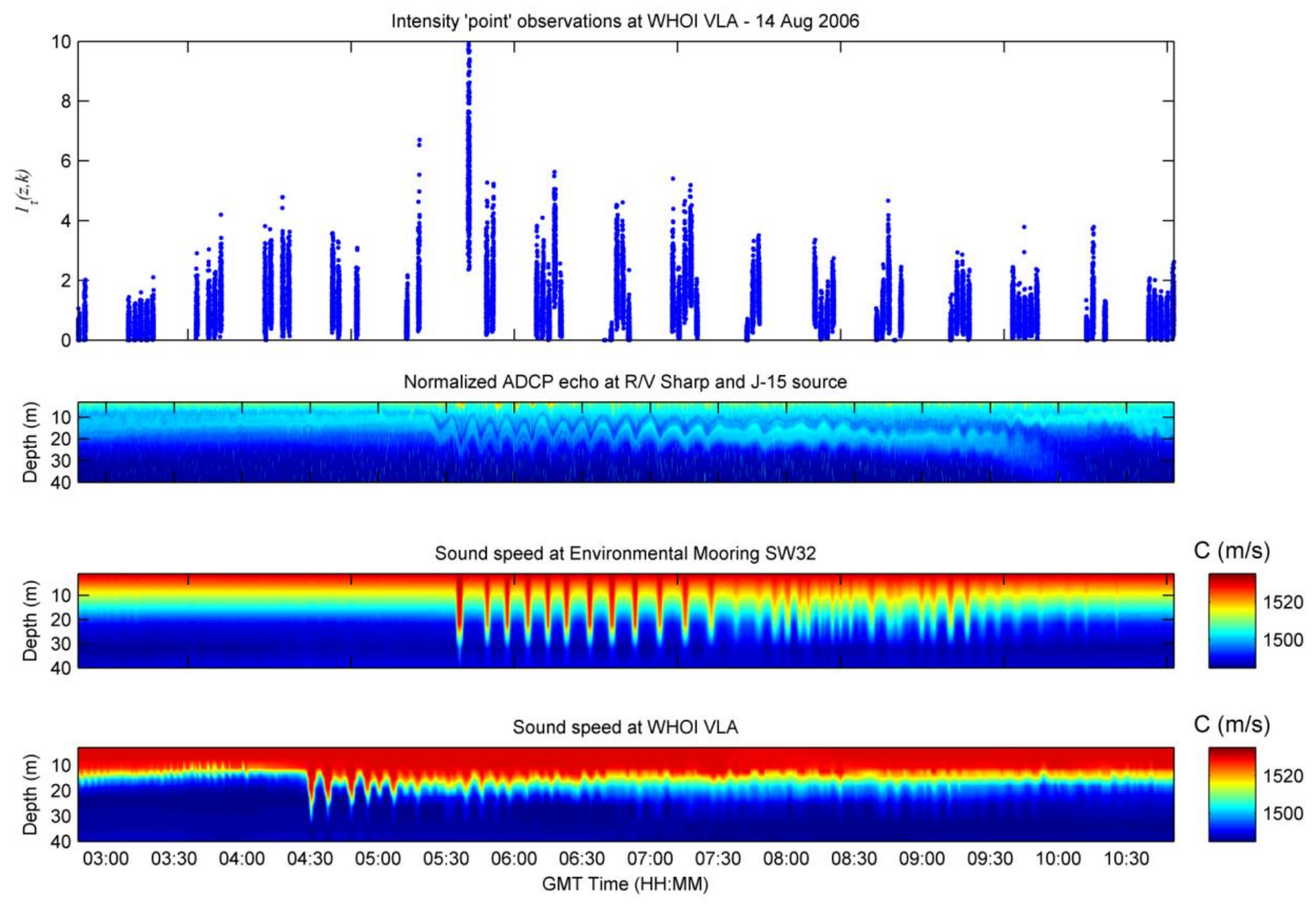

Figure A.8 Recalculated energy metrics after a $\underline{90 \%}$ noise threshold has been implemented. Ramping effect is still evident. 

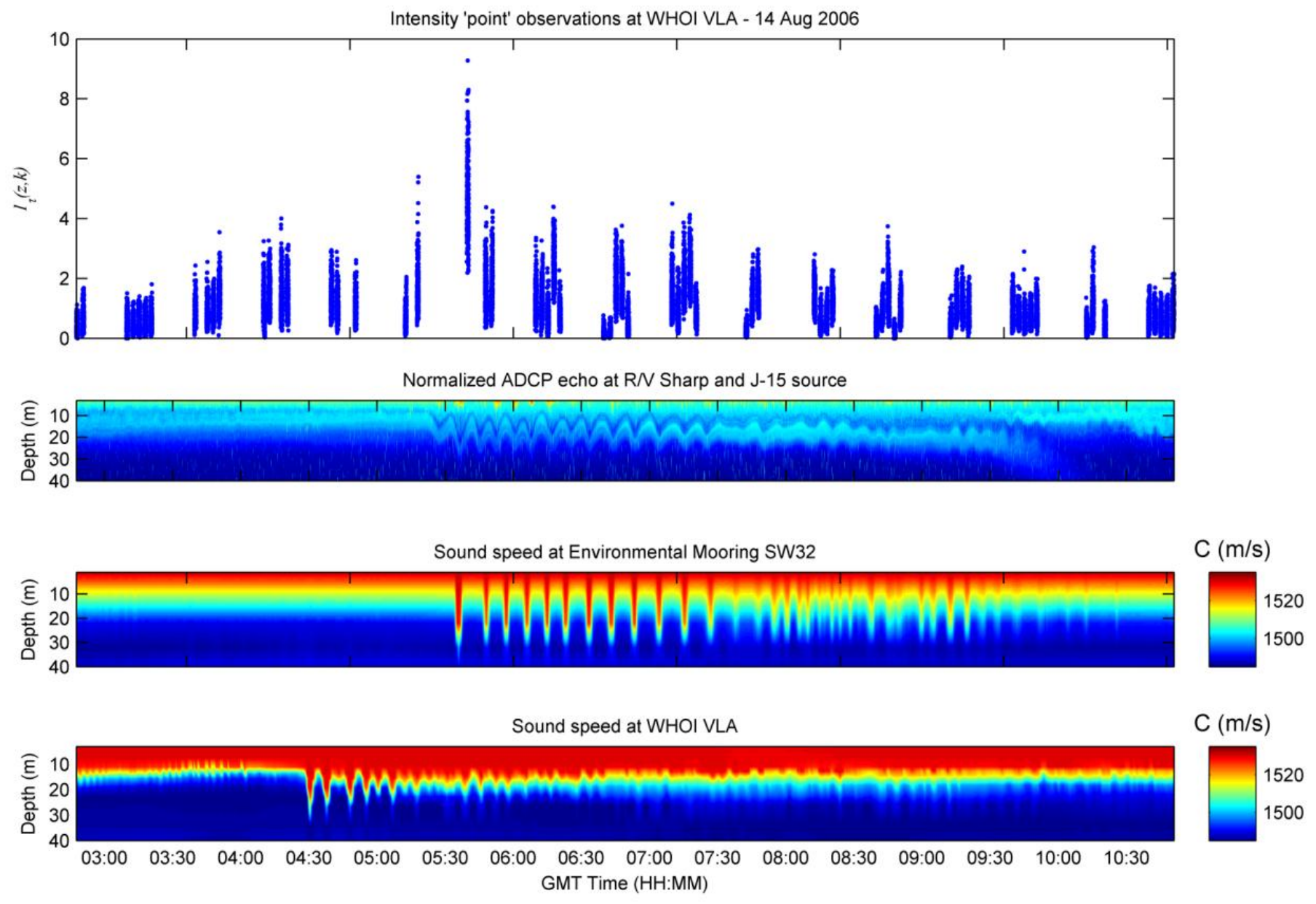

Figure A.9 Recalculated energy metrics after an $\underline{85 \%}$ noise threshold has been implemented. Ramping effect is still evident. 


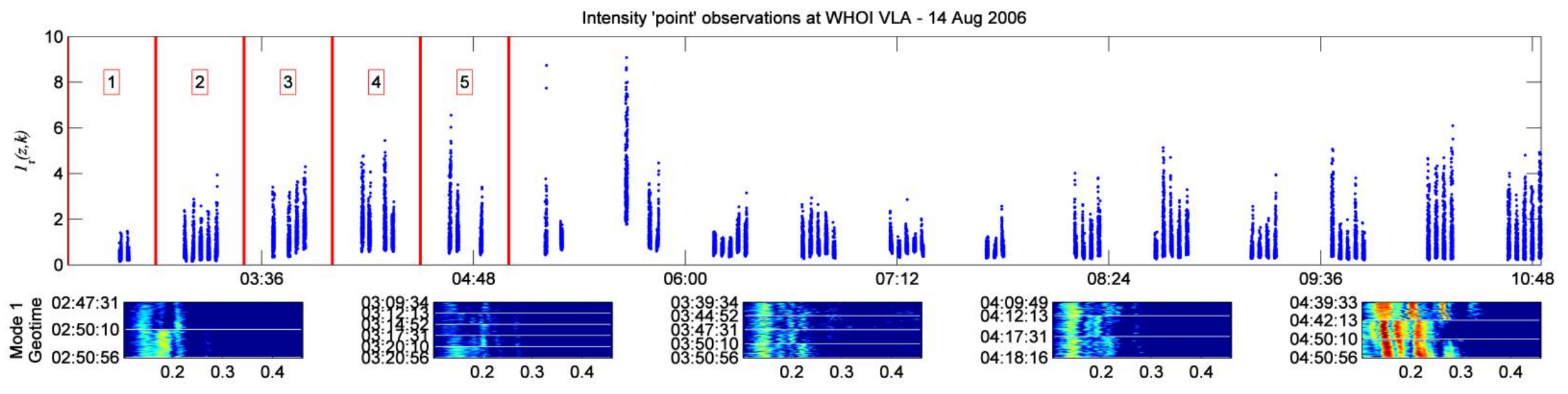

ร
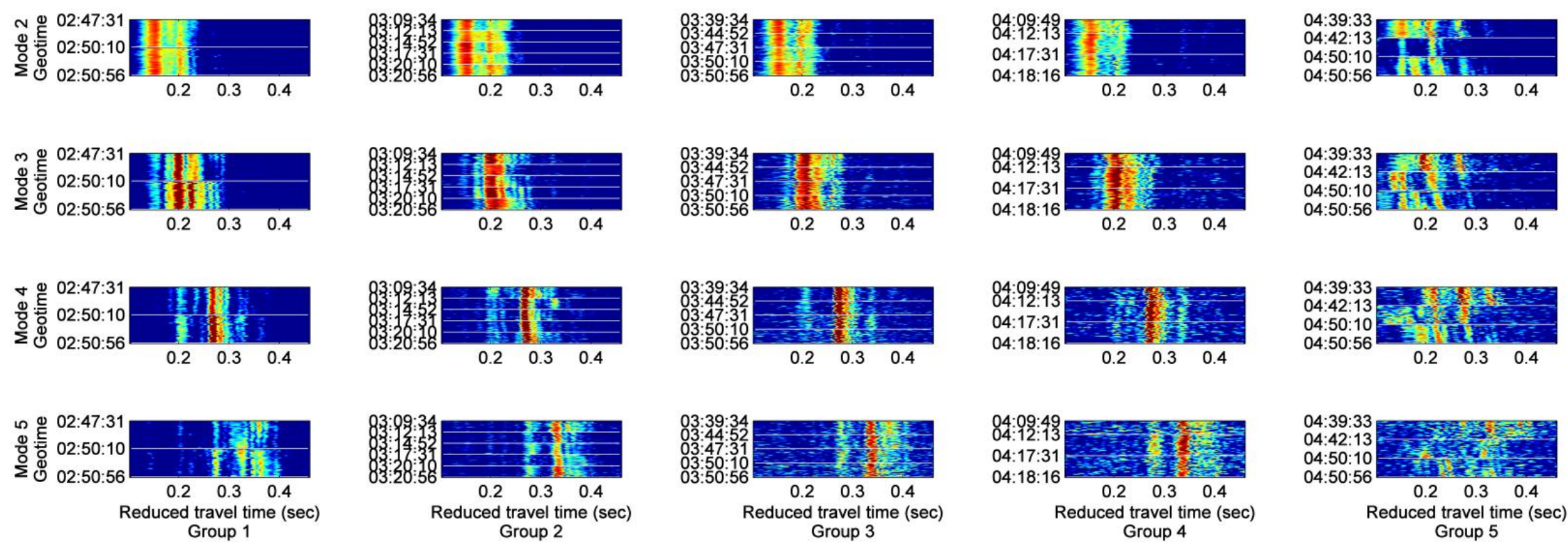

Figure A.10 Stacked time arrival plots for sections of Event 44 data where horizontal refraction may be occurring. 
Intensity "Point" Observations

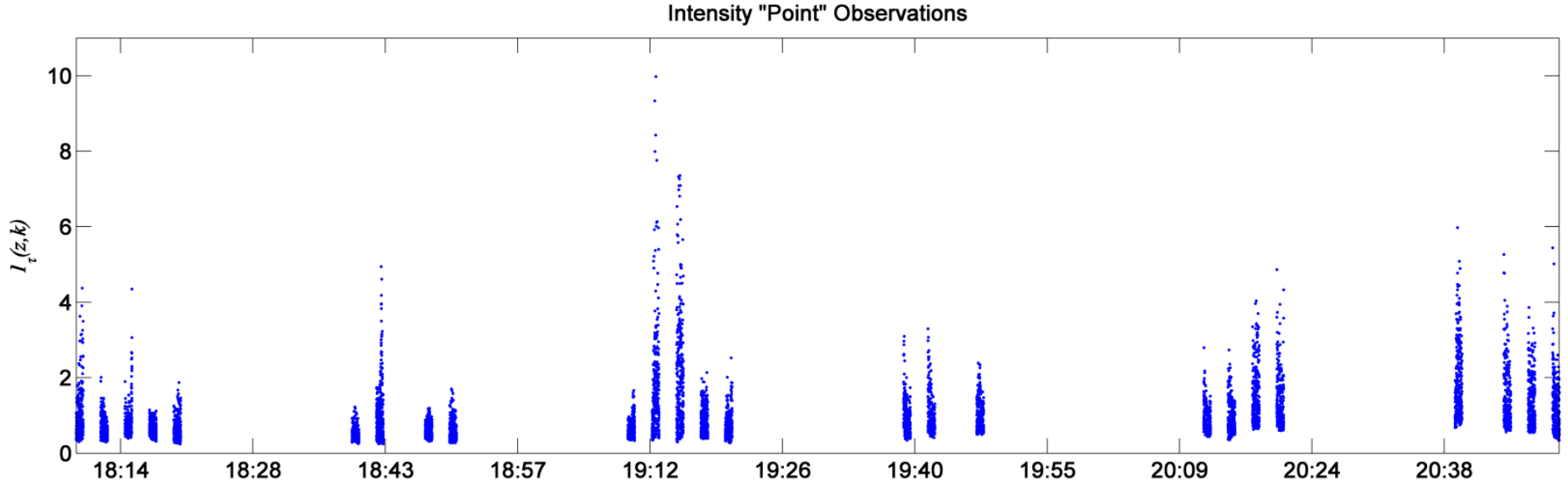

Normalized ADCP echo at R/V Sharp and J-15 source

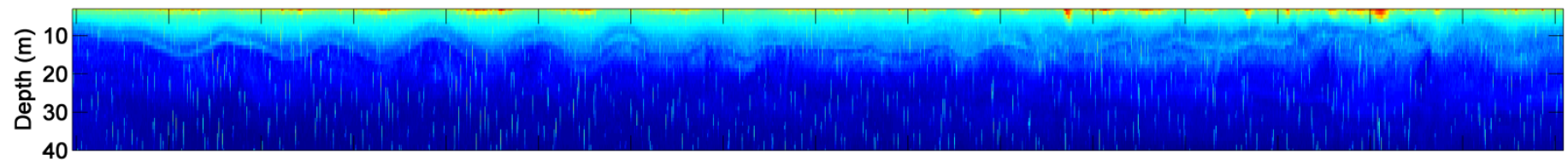

Sound speed at WHOI VLA

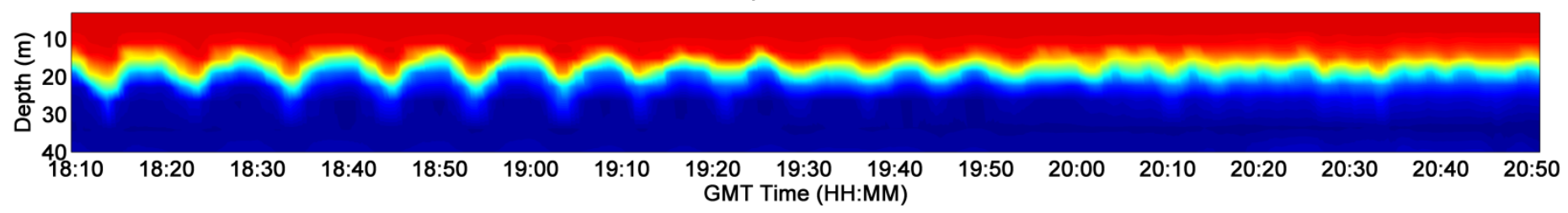

Figure A.11 "Point" observations for Event 45, which occurred on 14 Aug 2006 (top). NIW activity at source (center) and at receiving VLA (bottom). This dataset indicative of NIW interference across the entire acoustic track. 

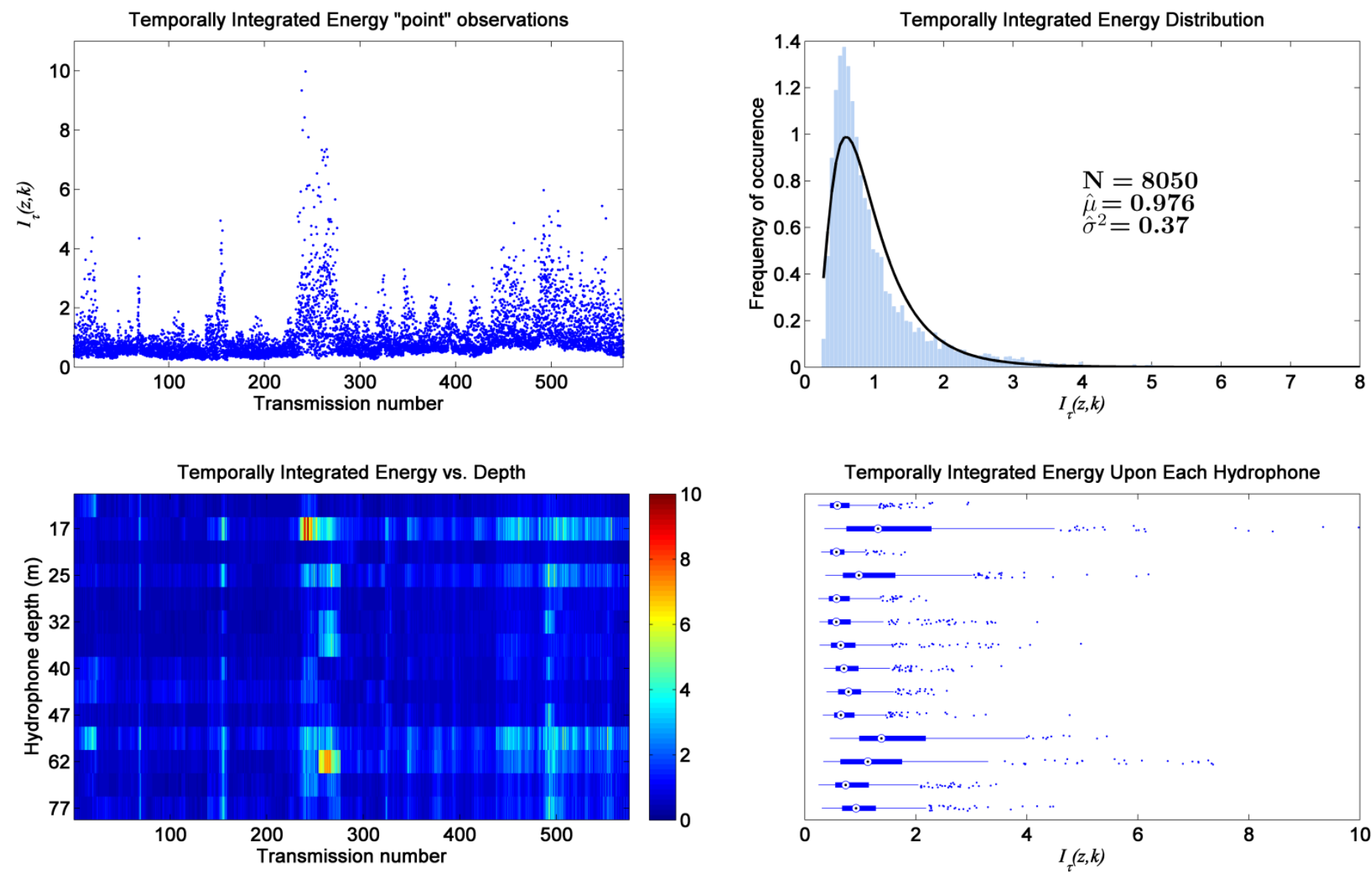

Figure A.12 Event 45 "point" observations are plotted versus transmission number (top left), and the data fits well to a lognormal distribution (tip right). Depth dependent variability is plotted using a normalized color scalogram versus transmission number (bottom left), or alternatively shown through a statistical boxplot of received energy upon each hydrophone (bottom right). 

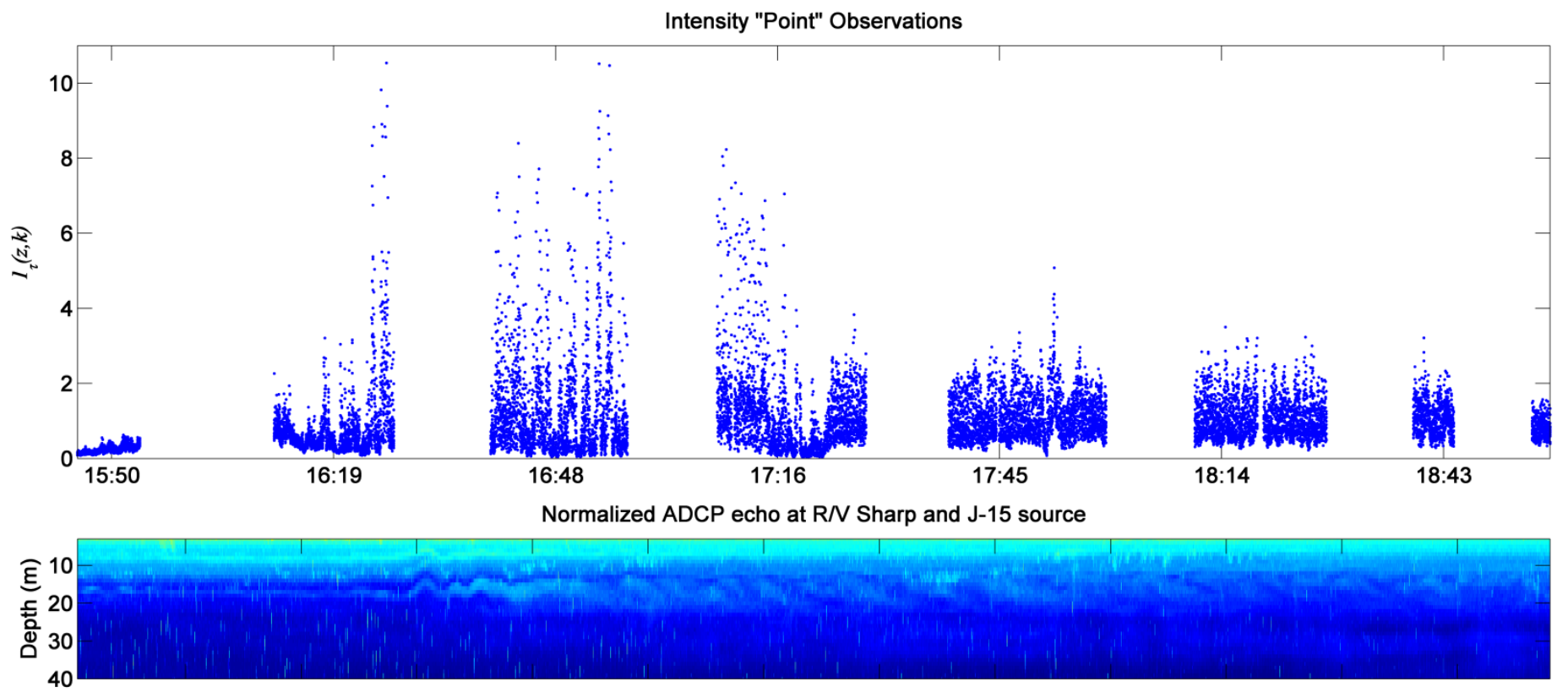

Sound speed at WHOI VLA

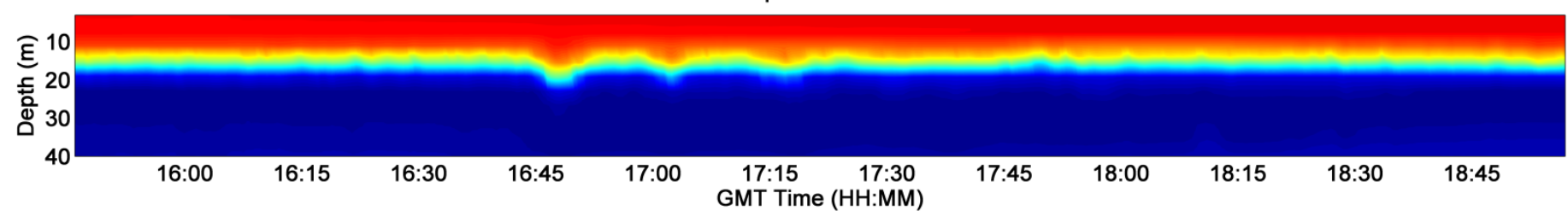

Figure A.13 "Point" observations for Event 46, which occurred on 16 Aug 2006 (top). NIW activity at source (center) and at receiving VLA (bottom). This dataset may indicate refraction prior to NIW activity, and also NIW interference. 

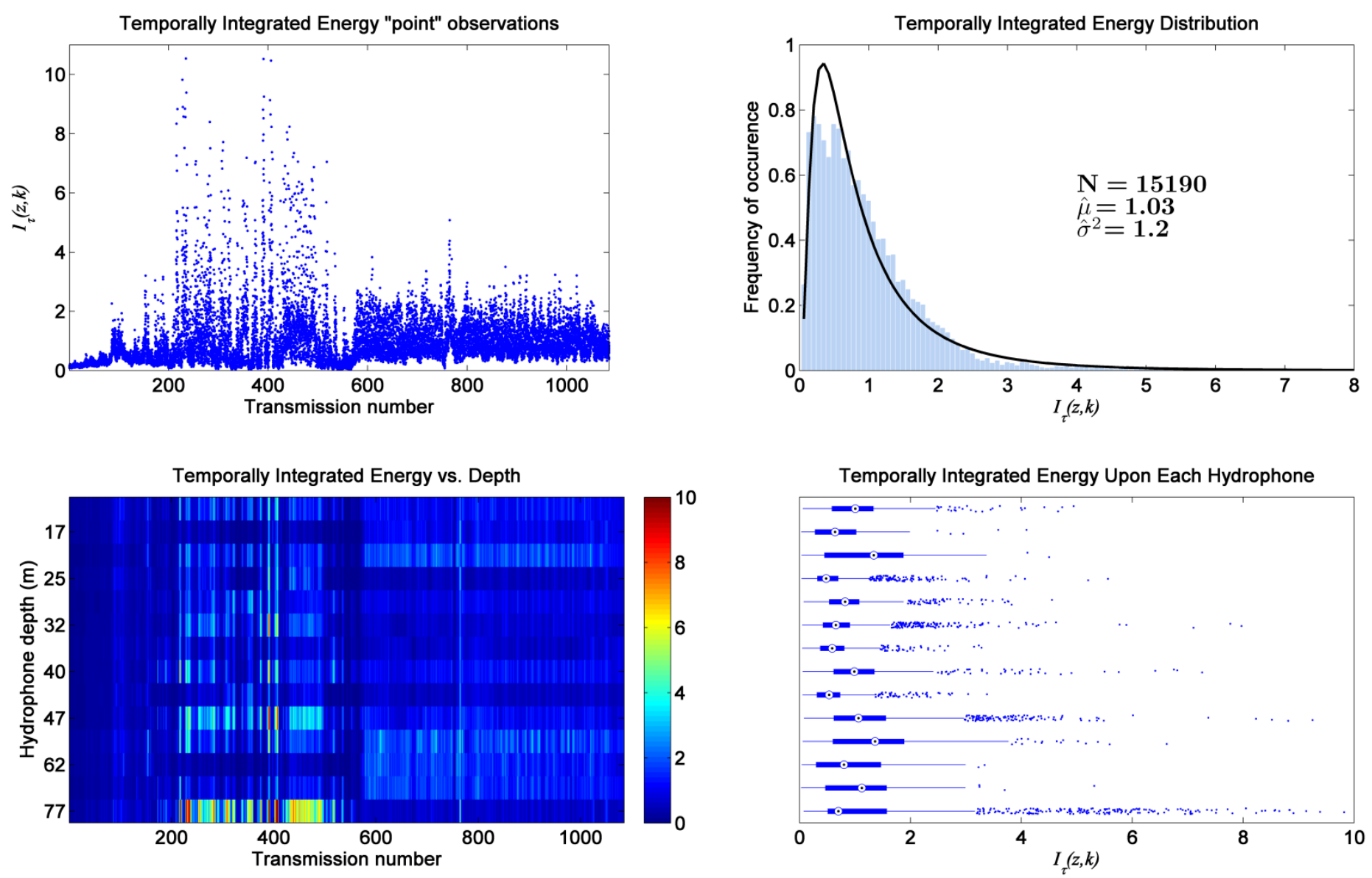

Figure A.14 Event 46 "point" observations are plotted versus transmission number (top left), and the data fits well to a lognormal distribution (tip right). Depth dependent variability is plotted using a normalized color scalogram versus transmission number (bottom left), or alternatively shown through a statistical boxplot of received energy upon each hydrophone (bottom right). 
Intensity 'point' observations at WHOI VLA - 16 Aug 2006
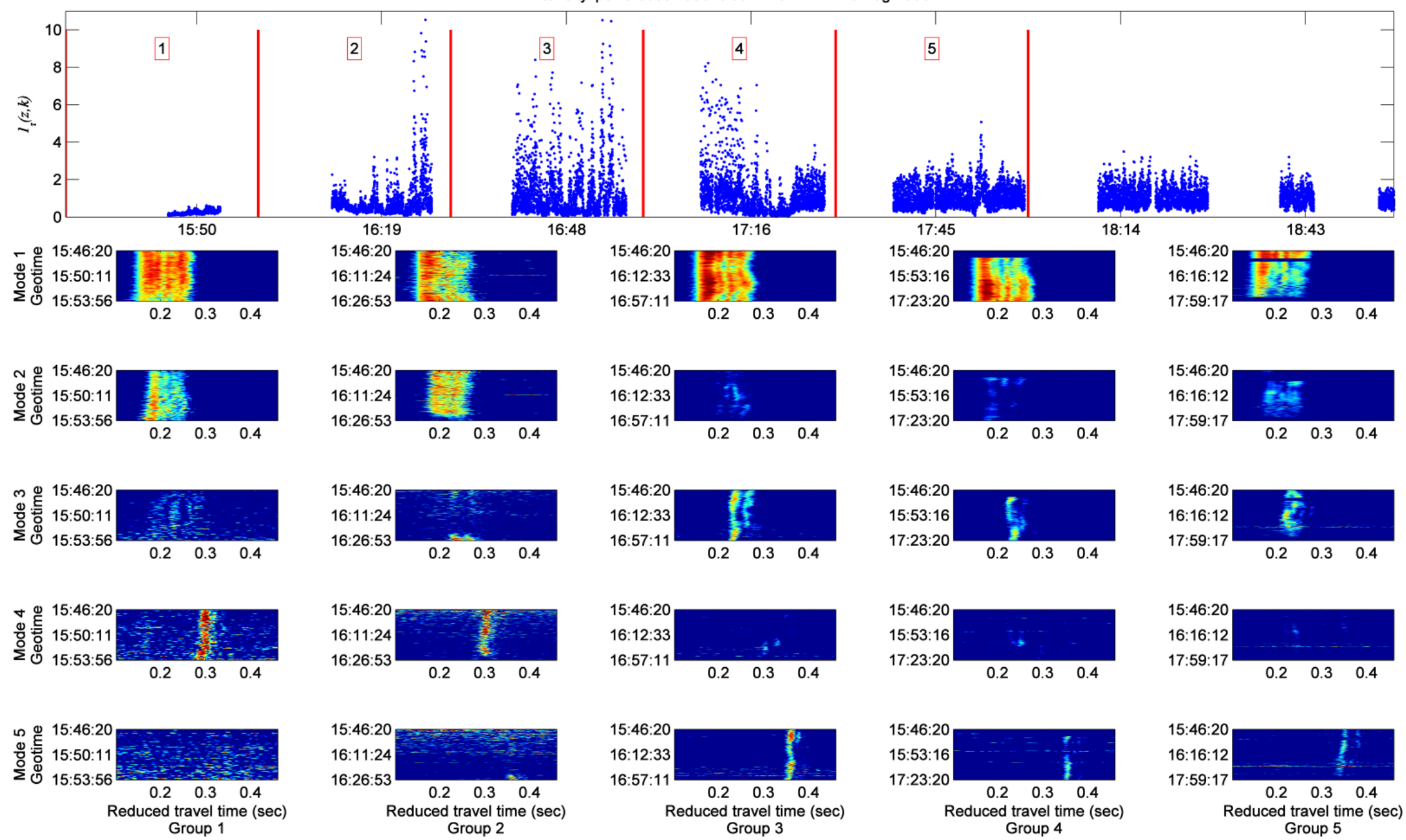

Figure A.15 Stacked time arrival plots for sections of Event $\mathbf{4 6}$ data where horizontal refraction may be occurring. 


\section{APPENDIX B. MMPE CONSIDERATIONS}

\section{B.1 Parabolic equation development in cylindrical coordinates}

The three-dimensional MMPE model is derived by representing a time-harmonic sound field $P$ in the cylindrical coordinate system $(r, z, \varphi)$ with radial frequency $\omega$ as

$$
P(r, z, \varphi, \omega t)=p(r, z, \varphi) e^{-j \omega t} .
$$

Substituted into the three-dimensional Helmholtz equation gives

$$
\frac{1}{r} \frac{\partial}{\partial r}\left(r \frac{\partial p}{\partial r}\right)+\frac{1}{r^{2}} \frac{\partial^{2} p}{\partial \varphi^{2}}+\frac{\partial^{2} p}{\partial z^{2}}+k_{0}^{2} n^{2}(r, z, \varphi) p=-4 \pi P_{0} \delta\left(\vec{x}-\vec{x}_{s}\right) .
$$

Here, the reference wavenumber $k_{0}$ is dependent on the reference sound speed $c_{0}$ by $k_{0}=\omega / c_{0}$. The acoustic index of refraction $n$, is determined by

$$
n(r, z, \varphi)=\frac{c_{0}}{c(r, z, \varphi)},
$$

with $c(r, z, \varphi)$ being the three-dimensional sound speed profile, and also the primary environmental water-column variable that induces perturbations into the acoustic field. The right hand side of (B.2) takes into account an acoustic source with pressure amplitude $P_{0}$ defined at a one meter reference distance $R_{0}$. The source is positioned at zero range and arbitrary depth (such that $r=r_{s}=0, z=z_{s}$ ), and the point source contribution is given by the Dirac delta function

$$
\delta(\vec{x})=\frac{1}{2 \pi r} \delta\left(z-z_{s}\right) \delta(r) .
$$


To simplify the Helmholtz equation, and to account for cylindrical (azimuthal) spreading, we let the pressure field be defined as a function of range and depth by

$$
p(r, z)=\frac{1}{\sqrt{r}} u(r, z)
$$

If we disregard the source term, substituting (B.5) into the Helmholtz equation (B.2) gives

$$
\frac{\partial^{2} u}{\partial r^{2}}+\frac{1}{r^{2}} \frac{\partial^{2} u}{\partial \varphi^{2}}+\frac{\partial^{2} u}{\partial z^{2}}+k_{0}^{2}\left[n^{2}+\frac{1}{4 k_{0}^{2} r^{2}}\right] u=0
$$

Assuming a far-field condition, we will neglect the last term in the above, as it drops off proportionally to $1 / r^{2}$. The second term allows for azimuthal coupling and is also often abandoned, an approach referred to as the uncoupled azimuth approximation (UNCA). ${ }^{1}$ However, we consider the azimuthal contribution an important aspect to our problem and retain its dependence.

To further simplify the Helmholtz equation, we introduce the differential operator notation

$$
\begin{aligned}
P_{o p} & =\frac{\partial}{\partial r} \\
Q_{o p} & =\sqrt{\mu+\varepsilon+v+1},
\end{aligned}
$$

where 


$$
\begin{aligned}
& \varepsilon=n^{2}-1 \\
& \mu=\frac{1}{k_{0}^{2}} \frac{\partial^{2}}{\partial z^{2}} \\
& \nu=\frac{1}{k_{0}^{2} r^{2}} \frac{\partial^{2}}{\partial \varphi^{2}},
\end{aligned}
$$

so that we can write the homogeneous form of the Helmholtz equation as

$$
\left[P_{o p}^{2}+k_{o p}^{2} Q_{o p}^{2}\right] u=0
$$

Equation (B.9) can be factored into a form that represents incoming and outgoing waves. However, we will assume that backscattered energy is negligible, and limit ourselves to an outward propagating field. This can be done by defining ${ }^{2}$

$$
u=Q_{o p}^{-1 / 2} \Psi
$$

where inclusion of the $Q_{o p}$ accounts for the first order WKBJ approximation. If we consider commutative terms between $P_{o p}$ and $Q_{o p}$ to be trivial, we can define a first order differential equation for outward radiating energy

$$
-j k_{0}^{-1} \frac{\partial \Psi}{\partial r}=Q_{o p} \Psi
$$

This is the parabolic acoustic wave equation (PE), and the starting point for a numerical model which implements a split-step Fourier approach. We define a PE field function

$$
\Psi=\psi(r, z, \varphi) e^{j k_{0} r}
$$

which is comprised of a slowly modulating envelope function and phase term dependent on the acoustic frequency. Written in terms of pressure, 


$$
p(r, z, \varphi)=P_{0} \sqrt{\frac{R_{0}}{r}} Q_{o p}^{-1 / 2} \psi(r, z, \varphi) e^{j k_{0} r}
$$

Substituting a normalized version of equation (B.13), where $|\psi|=1$ and $|p|=P_{0}$ at $r=R_{0}$, into the Helmholtz equation yields

$$
\frac{\partial \psi}{\partial r}=-j k_{0} \psi+j k_{0} Q_{o p} \psi
$$

If we introduce a Hamiltonian-like operator $H_{o p}$, which describes $Q_{o p}$ by its potential and kinetic energy components, and also defines the evolving PE field with range,

$$
H_{o p}=1-Q_{o p}
$$

we can rewrite (B.14) as

$$
\frac{\partial \psi}{\partial r}=-j k_{0} H_{o p} \psi=-j k_{0} \psi+j k_{0} Q_{o p} \psi
$$

Now, because we have cast the above problem in a form where the PE field is a vector in the $z$ dimension, we can represent the field as a function of increasing range with the use of a propagator function $\Phi(r)$

$$
\psi(r+\Delta r)=\Phi(r) \psi(r)
$$

The advantage of the SSF method is that each individual operator within $Q_{o p}$ or $H_{o p}$ can be multiplied in either the spatial ( $z$-space) or Fourier transform $\left(k_{z}\right.$-space) domain - the domain choice made to lessen computational burden. However, these operators in (B.7) and (B.8) are a combination of scalar and differential forms. Therefore, the operator 
terms must be separated, which requires an approximation to their joined dependence by the square-root (see equation (B.7)). To do this we treat the three-dimensional problem as a $N \times 2 D$ problem. We assume that azimuthal coupling is small, and can be accounted for by a binomial expansion term,

$$
Q_{o p} \approx \sqrt{\mu+\varepsilon+1}+\frac{1}{2} v=Q_{U N C A}+\frac{1}{2} v
$$

The often-used wide-angle approximation ${ }^{3}$ can then be employed to account for the UNCA approximation, given by

$$
H_{o p} \approx T_{o p}+U_{o p}+V_{o p}
$$

where

$$
\begin{aligned}
& T_{o p}=1-\left[1+\frac{1}{k_{0}^{2}} \frac{\partial^{2}}{\partial z^{2}}\right] \\
& U_{o p}=-[n-1] \\
& V_{o p}=-\frac{1}{2 k_{0}^{2} r^{2}} \frac{\partial^{2}}{\partial \varphi^{2}}
\end{aligned}
$$

Therefore (B.17) can be represented, and implemented, as a range-step marching algorithm,

$$
\psi(r+\Delta r) \approx e^{-j k_{0} \Delta r\left(T_{o p}+U_{o p}+V_{o p}\right)} \psi(r)
$$

To implement the algorithm, we rely on a two-dimensional Fourier transform convention

$$
\begin{aligned}
\psi(r, z, \varphi) & =\iint \hat{\psi}\left(r, k_{z}, k_{0} s\right) e^{j k_{z} z} e^{j k_{0} s \varphi} d\left(k_{0} s\right) d k_{z} \\
& =F F T^{2 D}\left\{\hat{\psi}\left(r, k_{z}, k_{0} s\right)\right\}
\end{aligned}
$$


Which allows us to transform the variable pairs $z \Leftrightarrow k_{z}$ and $\varphi \Leftrightarrow k_{0} s . U_{o p}$ is

numerically simple in the spatial domain. However both $V_{o p}$ and $T_{o p}$ are differential operators. Transforming them into the Fourier domain converts them to scalar operators,

$$
\begin{aligned}
& \hat{T}_{o p}\left(k_{z}\right)=1-\left[1-\left(\frac{k_{z}}{k_{0}}\right)^{2}\right]^{1 / 2} . \\
& \hat{V}_{o p}(r, s)=\frac{s^{2}}{2 r^{2}}
\end{aligned}
$$

Finally, the entire algorithm can be numerically implemented by a centered-step scheme, given by

$$
\begin{aligned}
\psi(r+ & \Delta r, z, \varphi)=e^{-j k_{0} \Delta r U_{o p}(r, z, \varphi)} \\
& \times F F T^{2 D}\left\{e^{-j k_{0} \Delta r\left[\hat{T}_{o p}\left(k_{z}\right)+\hat{V}_{o p}(r, s)\right]} \times \operatorname{IFFT}^{2 D}\{\psi(r, z, \varphi)\}\right\} .
\end{aligned}
$$

\section{B.2 Frequency sampling considerations}

In this section we discuss the consequence of possibly under-sampling the PE source bandwidth when calculating our energy metric $I_{f}$. As previously mentioned, even thirty computers working full-steam for one full week could not sample our problem at sufficient frequency intervals. To better understand this limitation, we have taken one NIW position, a refraction scenario, and have sampled at a finer granularity of $6.25 \mathrm{~Hz}$ versus $12.5 \mathrm{~Hz}$.

Figure B.1 shows $I_{f}$ metrics when sampling at $25 \mathrm{~Hz}$ versus $6.25 \mathrm{~Hz}$ to show worseand better-case scenarios compared to what we have accomplished at $12.5 \mathrm{~Hz}$ sampling. Both the point measurements and depth-dependence plots show a smoothing of the 
energy field at the finer frequency resolution. Figure B.2 expands the frequency

dependence of $I_{f}$ to show the intricate pattern of peaks and nulls at each cross-range point - this is shown at four depths in the water column as representative traces at $15 \mathrm{~km}$ range. Figure B. 3 shows a single cross-range point at $0.2 \mathrm{~km}$, corresponding to the peak refracted energy in Figure B.2. We can confirm that we are under-sampling, missing both peaks and valleys in our integration under the frequency curve (equation (3.22)). On the positive, even the worse-case $25 \mathrm{~Hz}$ sampling captures the overall envelope of the better-case scenario. This problem would remain "under-sampled" until enough points were calculated for an inverse Fourier transform, so that we could synthesize an actual time arrival.

\section{B.3 Alternative PDFs to PE data}

Figure B.4 shows other possible PDF fits to the overall data originally shown in Figure 3.21. A probability plot for these fits referenced to the lognormal distribution is also shown - this type of plot is often used to compare and choose between different PDFs. While, the lognormal does not fit perfectly at the tails, it performs better than other choices. The Gamma distribution performs well at the low end $(\ll 1)$, but performs quite poorly at the high end $(\gg 1)$.

\section{B.4 Modal excitation}

The data presented throughout Manuscript III assumes a source depth near the seafloor, which failed to sufficiently excite Mode 1 . To investigate the differences in modal excitation, similar refraction, defocusing, and focusing scenarios were recreated with the source positioned at 45 meters depth - chosen to excite Mode 1 regardless of a soliton being coincident with the source. Mode 1 does show signs of refraction, 
defocusing, and focusing, interference. However, it is slight compared to the higher order modes, and tends to "barrel through" the NIW more easily. Therefore, in Event 44, because the higher order modes were excited in the first place, refraction effects tend to dominate.

\section{REFERENCES AND LINKS}

1. K. B. Smith and F. D. Tappert, "Horizontal refraction and the uncoupled azimuth approximation," 343 (2004).

2. $\quad$ F. D. Tappert, "The parabolic approximation method," Wave propagation and underwater acoustics, Lecture Notes in Physics 70, 224-287 (1977).

3. D. J. Thomson and N. R. Chapman, "A wide-angle split-step algorithm for the parabolic equation," J. Acoust. Soc. Am. 93 (2627) (1993). 


\section{FIGURES}
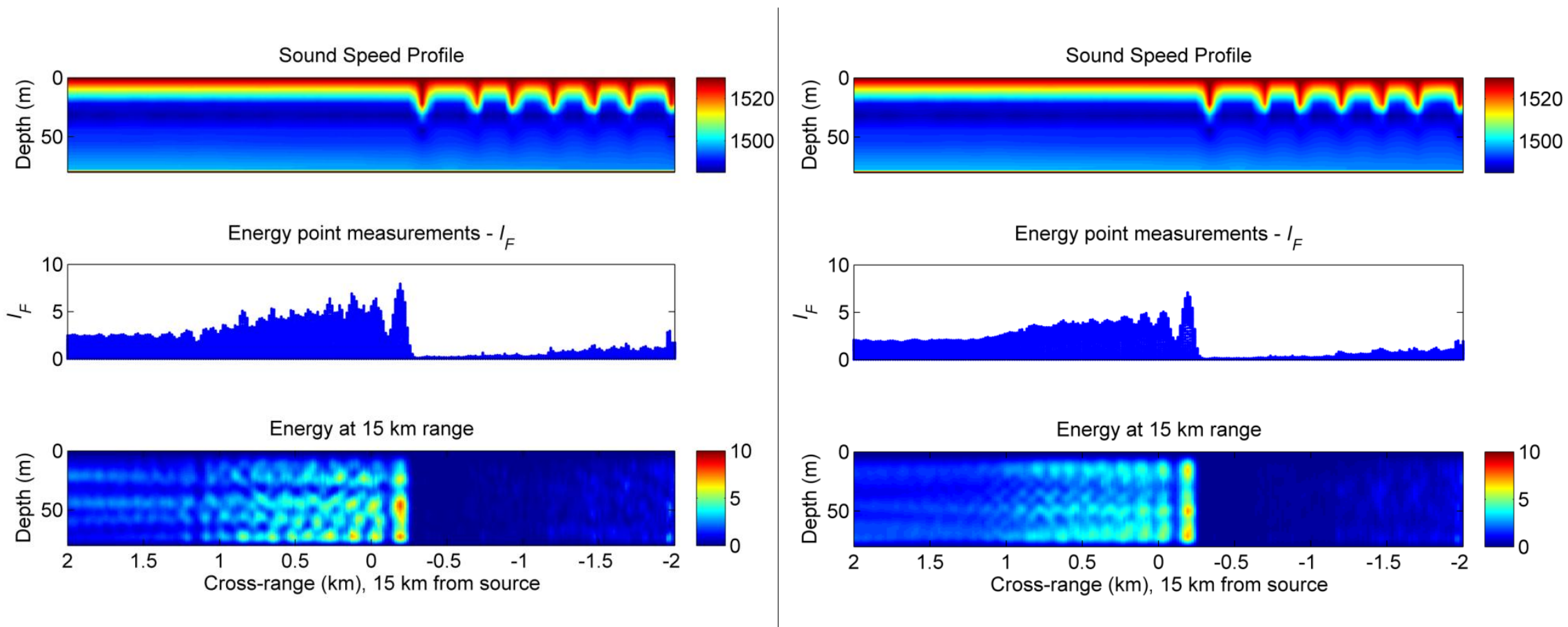

Figure B.1 Impact upon $I_{f}$ calculations based upon frequency sampling of signals' overall bandwidth. $25 \mathrm{~Hz}$ sampling (left) versus 6.25 Hz sampling (right). 
At 16 meters depth
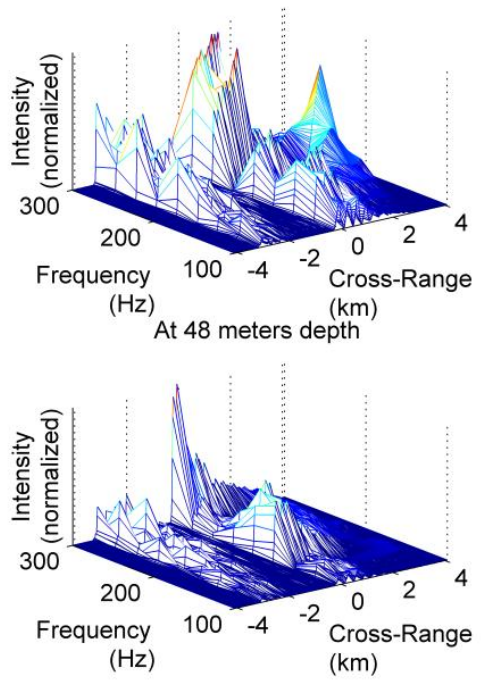

At 32 meters depth
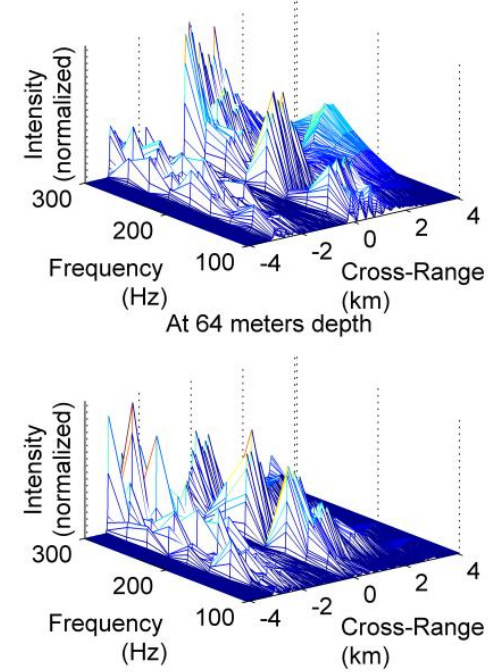

At 16 meters depth
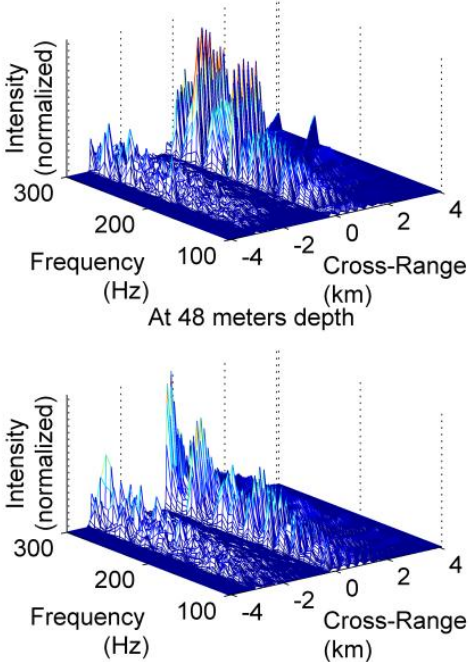

At 32 meters depth
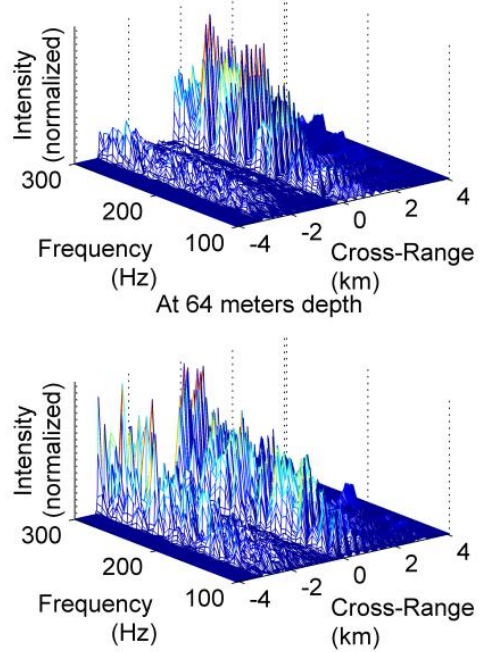

Figure B.2 Frequency dependent intensity versus cross-range at four arbitrary depths. $25 \mathrm{~Hz}$ sampling (left) fails to capture some of the frequency-dependent structure shown by $6.25 \mathrm{~Hz}$ sampling (right). Figures correspond to those shown in Figure B.1 
At 16 meters depth, $-0.2 \mathrm{~km}$ cross-range

At 32 meters depth, $-0.2 \mathrm{~km}$ cross-range
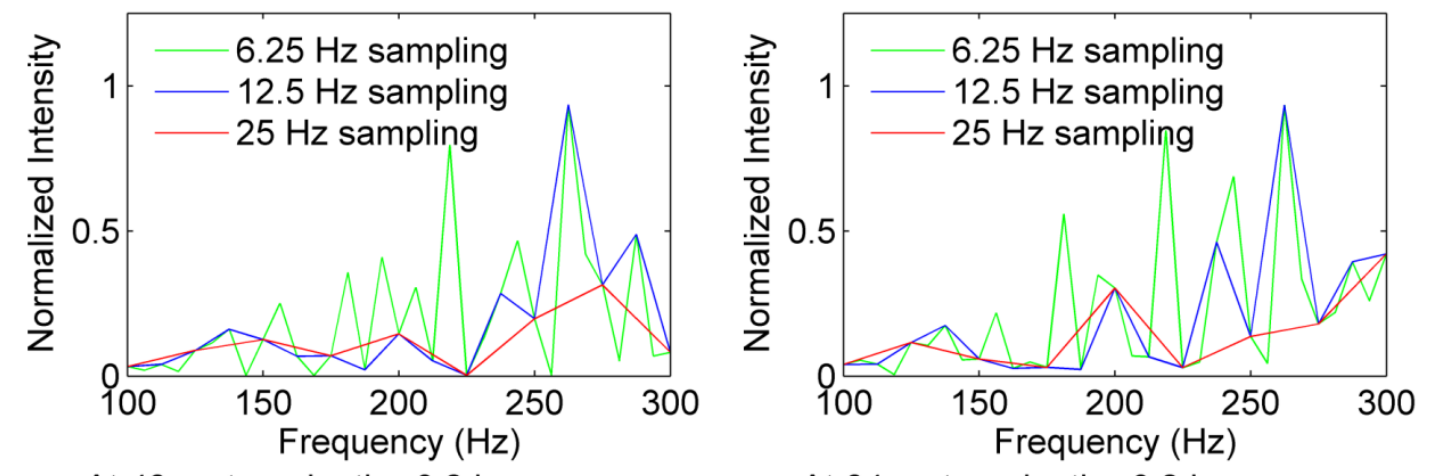

At 48 meters depth, $-0.2 \mathrm{~km}$ cross-range

At 64 meters depth, $-0.2 \mathrm{~km}$ cross-range
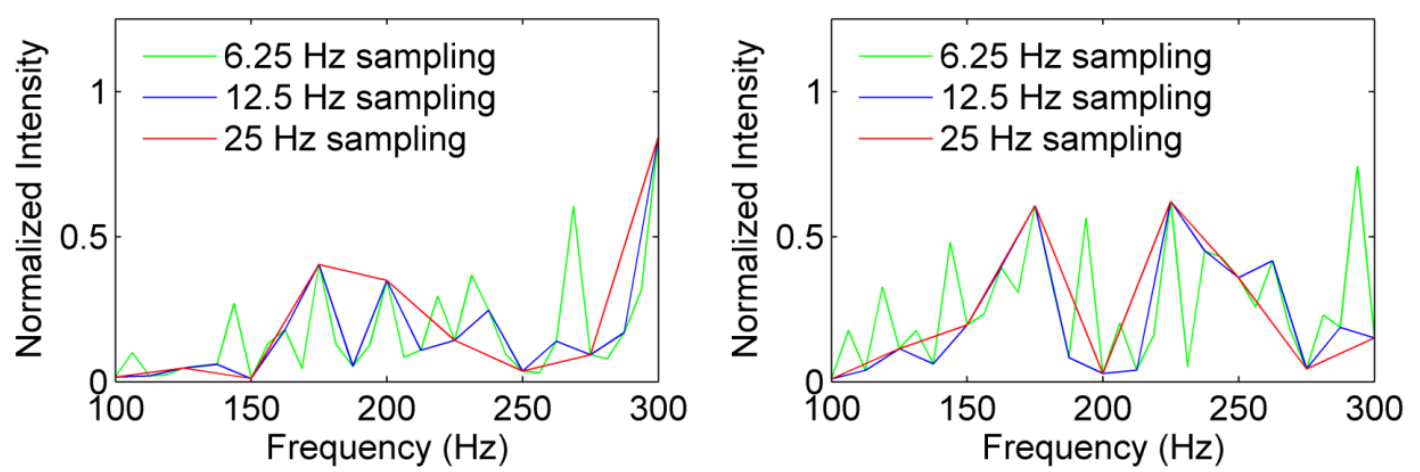

Figure B.3 Single trace corresponding to cross-range of $\mathbf{- 0 . 2} \mathbf{~ k m}$, which is the point of maximum refracted energy, previously shown in Figure B.1and Figure B.2. Finer sampling captures spikes and fades that are not apparent in the under-sampled profiles. 

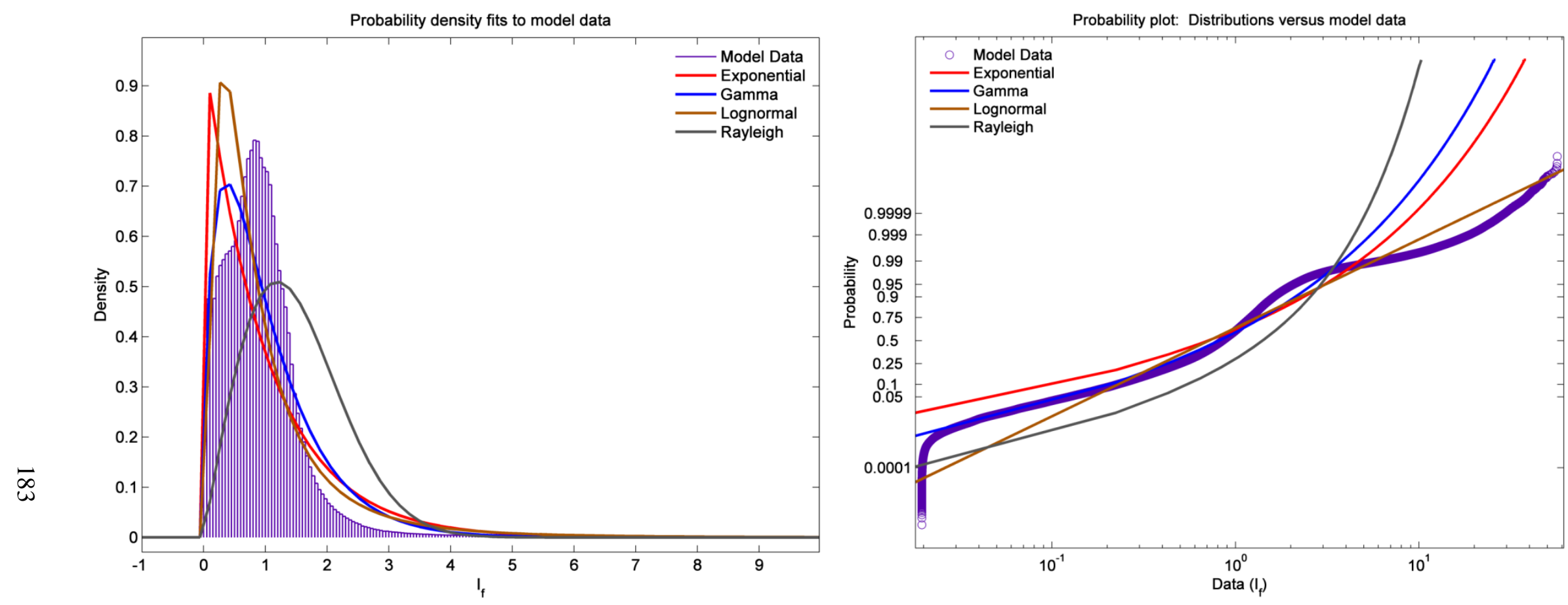

Figure B.4 Various PDFs fit to the ensemble modeled dataset (left). A probability plot helps interpret which distributions are better choices than others. 

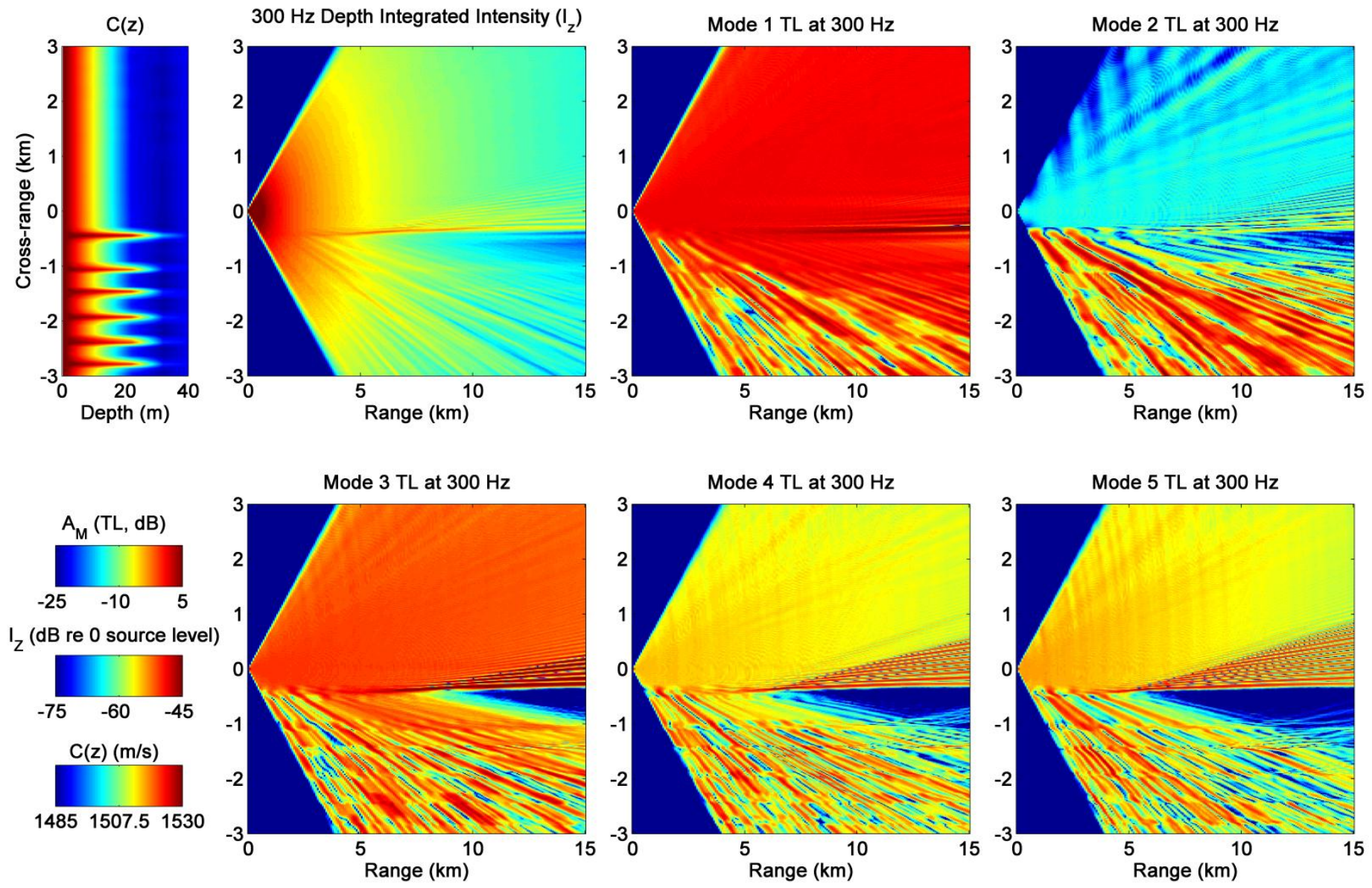

Figure B.5 Depth integrated intensity and modal decomposition of the PE field for modes 1-5 for a $300 \mathrm{~Hz}$ source. Sound speed input creates a refraction scenario. Source depth changed to $z=45$ meters to better excite Mode 1 . 

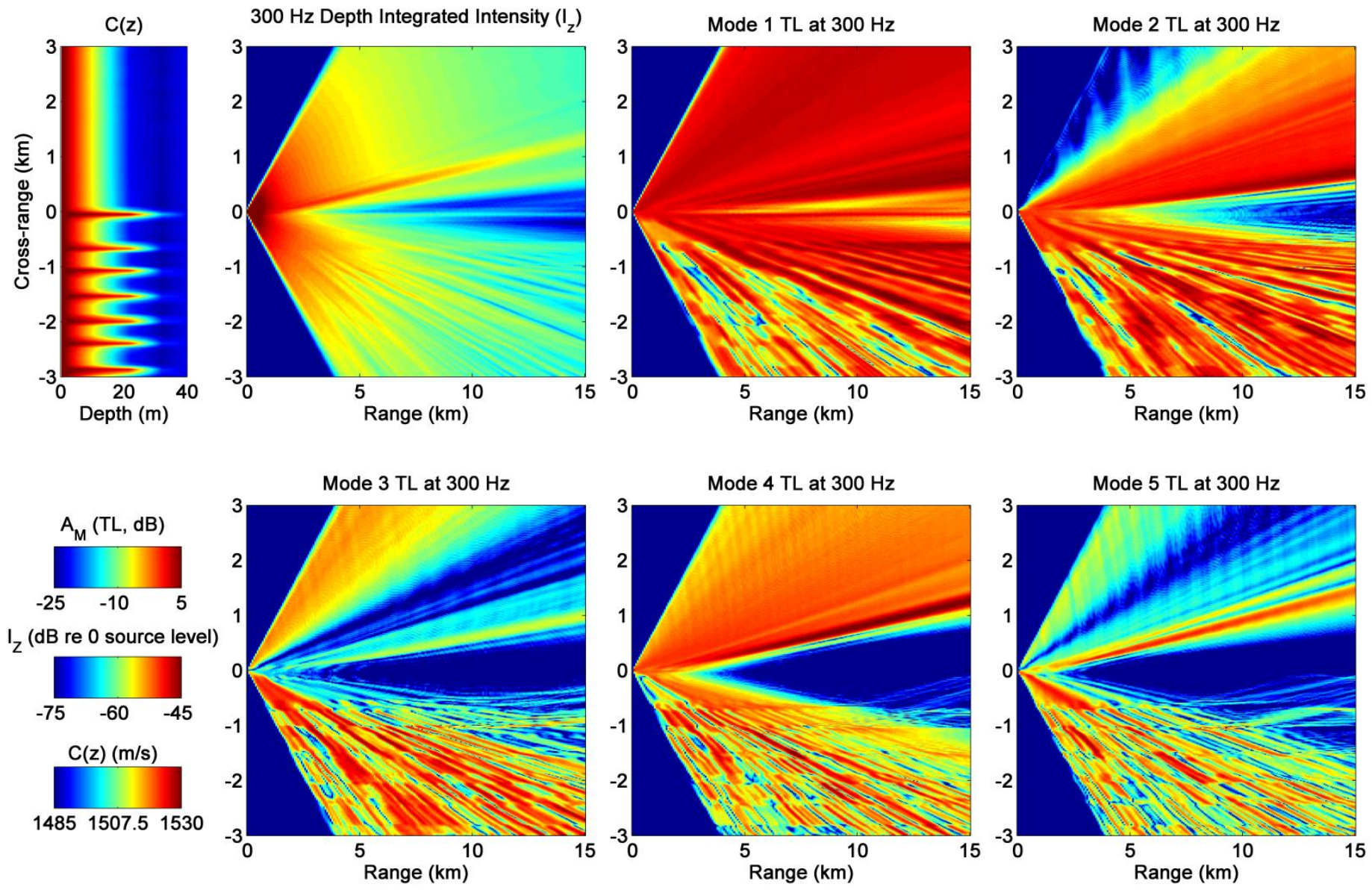

Figure B.6 Depth integrated intensity and modal decomposition of the PE field for modes 1-5 for a $300 \mathrm{~Hz}$ source. Sound speed input creates a defocus scenario. Source depth changed to $z=45$ meters to better excite Mode 1 . 

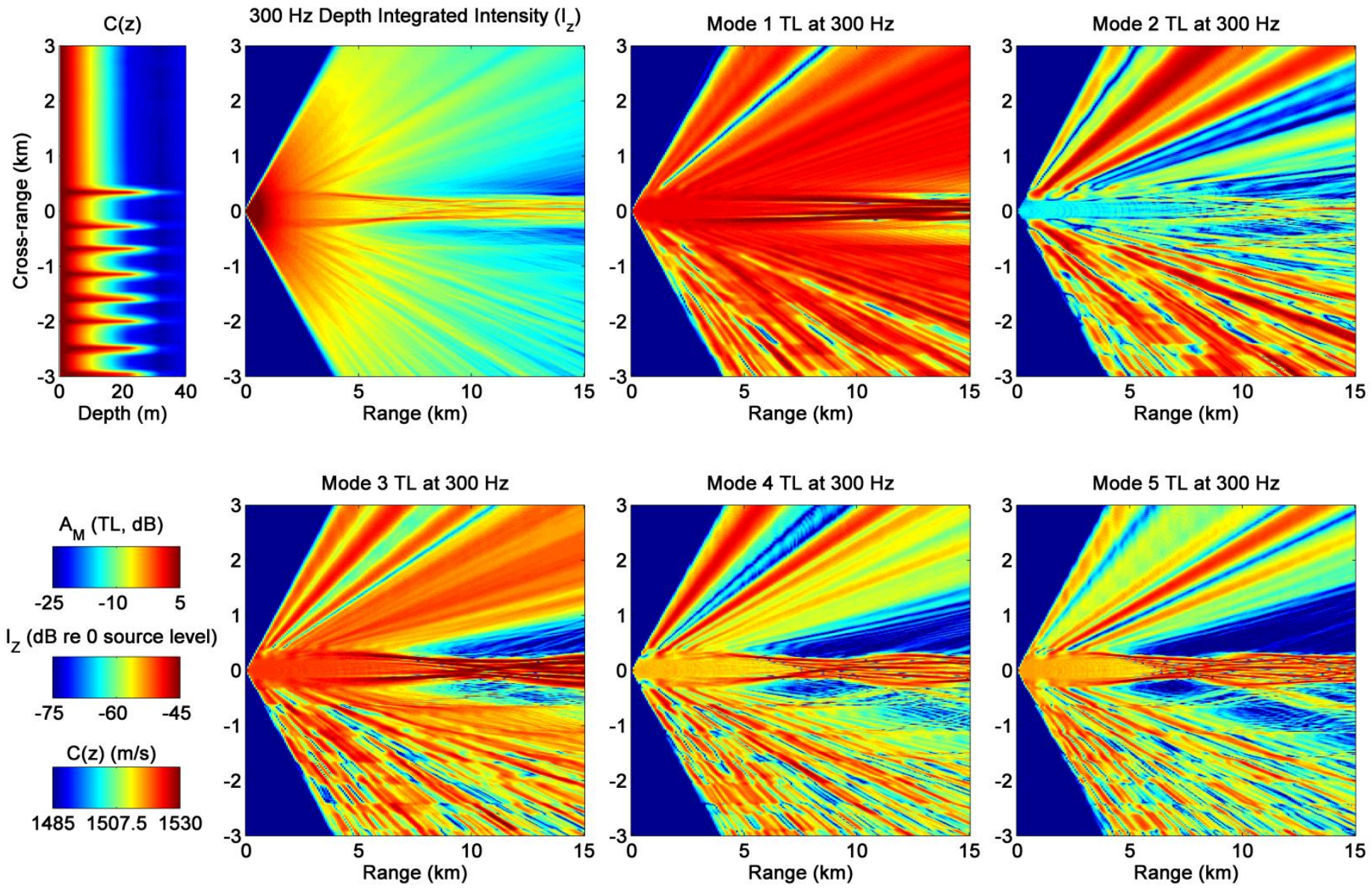

Figure B.7 Depth integrated intensity and modal decomposition of the PE field for modes 1-5 for a $300 \mathrm{~Hz}$ source. Sound speed input creates a focus scenario. Source depth changed to $z=45$ meters to better excite Mode 1 . 


\section{APPENDIX C. BROADBAND MODAL DECOMPOSITION \\ C.1 Problem, assumptions, and theory}

This appendix details the modal decomposition techniques used to treat experimental data received on the WHOI VLA. For this problem, we assert the following:

- We have general knowledge of the experimental configuration, including locations of the source and receiving VLA.

- We can recreate the original transmitted signal, which is a broadband LFM chirp, spanning between 50-450 Hz.

- We simplify the ocean environment by assuming a flat sea bottom, and a layered seafloor with non-varying geoacoustic parameters.

- We assume the sound speed profile at the receiving VLA is valid across the entire acoustic track, and is sufficient for calculating time-varying modal eigenvalues due to water column perturbations.

In treating this problem, we address the following:

- Our signal is broadband, therefore we must account for frequency dependence in the modal decomposition.

- The signal has low SNR, and the presence of noise. Therefore, we wish to implement a matched filter.

- The measured data shows modal dispersion. We address possible ways to account for dispersion, but further work remains in this area. 
From JKPS, ${ }^{1}$ one can describe the pressure field due to a point source in cylindrical geometry as

$$
p(r, z)=\frac{j}{4 \rho\left(z_{s}\right)} \sum_{m=1}^{\infty} \Psi_{m}\left(z_{s}\right) \Psi_{m}(z) H_{0}^{(1)}\left(k_{r m} r\right),
$$

where $\rho\left(z_{s}\right)$ is the medium density at source depth $z_{s}, \Psi_{m}(z)$ the depth dependent mode function, and $H_{0}^{(1)}\left(k_{r m} r\right)$ the Hankel function with horizontal wavenumber $k_{r m}$ and radial distance $r$. If we consider the asymptotic approximation to the Hankel function, we can rewrite (C.1) as

$$
p(r, z)=\frac{j}{\rho\left(z_{s}\right) \sqrt{8 \pi r}} e^{-j \pi / 4} \sum_{m=1}^{\infty} \Psi_{m}\left(z_{s}\right) \Psi_{m}(z) \frac{e^{j k_{r m} r}}{\sqrt{k_{r m}}}
$$

Within the summation in (C.2), each mode consists of an initial excitation $\Psi_{m}\left(z_{s}\right)$, a depth-dependent modal amplitude $\Psi_{m}(z)$, and a phase term proportional to $e^{j k_{r m} r}$.

Our goal is to cast the problem into an input-output problem that follows traditional LTI signal processing methods. We assume that the ocean medium can be described by a transfer function $H(f)$, where $f$ is the frequency in Hertz, and we presume each mode has a unique $H_{m}(f)$. Our input signal is derived from a receiving VLA with $N$ elements, each at depth $z_{n}$. We can represent the input pressure signal in array form as $\left[x_{z_{n}}(t)\right]$, with a Fourier transform $\left[X_{z_{n}}(f)\right]$, so that $\left[x_{z_{n}}(t)\right] \Leftrightarrow\left[X_{z_{n}}(f)\right]$. 


$$
\left[x_{z_{n}}(t)\right]=\left[\begin{array}{c}
x_{z_{1}}(t) \\
x_{z_{2}}(t) \\
\vdots \\
x_{z_{N}}(t)
\end{array}\right] \Leftrightarrow\left[X_{z_{n}}(f)\right]=\left[\begin{array}{c}
X_{z_{1}}(f) \\
X_{z_{2}}(f) \\
\vdots \\
X_{z_{N}}(f)
\end{array}\right]
$$

where the above rows are evaluated at hydrophone depths

$$
\left[z_{n}\right]=\left[\begin{array}{c}
z_{1} \\
z_{2} \\
\vdots \\
z_{N}
\end{array}\right]
$$

Equation (C.5) shows our overall approach to extracting the modal time series component $y_{m}(t)$ from the original $\left[x_{z_{n}}(t)\right]$. After transferring the time series into the frequency domain, we filter the signal into a frequency band of interest using a band-pass filter $B P(f)$ and also implement a match-filter $M F(f)$ to increase signal gain. At the same time we apply the transfer function $H_{m}(f)$, which can also be interpreted as a modal beamformer. Once these operations have been performed, we obtain an output signal $\left[Y_{z_{n}}(f)\right]$ upon each phone. These signals can be returned to the time domain through an inverse Fourier transform, and summed over depth, resulting in the time series component for each particular mode. 


$$
\begin{aligned}
{\left[\begin{array}{c}
x_{z_{1}}(t) \\
x_{z_{2}}(t) \\
\vdots \\
x_{z_{N}}(t)
\end{array}\right] \stackrel{F F T}{\Rightarrow}\left[\begin{array}{c}
X_{z_{1}}(f) \\
X_{z_{2}}(f) \\
\vdots \\
X_{z_{N}}(f)
\end{array}\right] \times\left[\begin{array}{c}
B P(f) \\
B P(f) \\
\vdots \\
B P(f)
\end{array}\right] \times\left[\begin{array}{c}
M F(f) \\
M F(f) \\
\vdots \\
M F(f)
\end{array}\right] \times\left[\begin{array}{c}
H_{z_{n} m}(f) \\
H_{z_{n} m}(f) \\
\vdots \\
H_{z_{N} m}(f)
\end{array}\right]=\left[\begin{array}{c}
Y_{z_{1} m}(f) \\
Y_{z_{2} m}(f) \\
\vdots \\
Y_{z_{N} m}(f)
\end{array}\right] } \\
{\left[\begin{array}{c}
Y_{z_{n} m}(f) \\
Y_{z_{n} m}(f) \\
\vdots \\
Y_{z_{N} m}(f)
\end{array}\right] \stackrel{I F F T}{\Rightarrow}\left[\begin{array}{c}
y_{z_{1} m}(t) \\
y_{z_{2} m}(t) \\
\vdots \\
y_{z_{N} m}(t)
\end{array}\right] \rightarrow \begin{array}{l}
\rightarrow \\
\rightarrow
\end{array} }
\end{aligned}
$$

In the above, multiplication is performed element-wise. Additionally, both the band-pass and match filtering are optional operations, but create greater confidence in our signal extraction given the slightly noisy environment.

To calculate the transfer function (or modal beamformer) $H_{m}(f)$, we rely on the KRAKEN normal mode program ${ }^{2}$ to calculate the modal eigenvalues, frequency dependent mode shapes and group velocities. The MATLAB-KRAKEN interface was graciously provided by Ying-Tsong Lin (WHOI). It relies upon the local sound speed at the WHOI VLA, and assumes a preliminary ten-layer bottom model courtesy of Ross Chapman (Univ. of Victoria). $H_{m}(f)$ is calculated such that each row represents a frequency dependent weighting at each array element. This weight consists of a modal amplitude $\Psi_{m}(z)$ and phase factor $e^{j k_{m m} r}$. A general representation for these weights is shown in Figure C.1, where in the frequency domain, the weights create a "surface." The magnitude calculated by the local mode shapes $\left\|H_{m}(z, f)\right\|=\left\|\Psi_{m}(z, f)\right\|$, and the phase calculated by the modal phase term $\measuredangle H(z, f)=\frac{e^{j k_{m m} r}}{\sqrt{k_{r m}}}$. Figure C.2 shows the one-sided amplitude weights used to calculate chirp mode arrivals in Figure 2.12 and Figure 2.13 
Unfortunately, incorporating phase information, which ideally would account for dispersion, proves to be difficult. Before introducing this information into the modal beamformer, we realize that phase accumulates over the source-receiver path, and that in our experimental geometry, the ocean and sea bottom vary, causing range dependence in $k_{r m}$. Does an average value for $k_{r m}$ suffice? The top panel of Figure C.3 shows

KRAKEN estimates for $k_{r m}$ for the first five modes. Suppose we can accept a tolerance for $\Delta k_{r m} r$ of $\pi / 4$, where $r$ is $R=15 \mathrm{~km}$. This criterion mandates the KRAKEN calculation be accurate to within $\Delta k_{r m} \leq \frac{\pi}{4 R} \approx 5.2 \times 10^{-5}$ ! Unfortunately, we cannot estimate $k_{r m}$ simply by relying upon the KRAKEN outputs. Work in this area can be continued, where one would use KRAKEN outputs as initial values in an inversion scheme that would estimate $k_{r m}$ within sufficient tolerance.

Another option to account for dispersion is to assume that mode arrivals act accordingly to their group velocities profiles. Consequently, we can create a modedependent matched filter. Let us denote our original transmitted signal as $s(t)$, with Fourier transform pair $S(f)$. Typically, a match-filter implementation in the frequency domain is carried out by

$$
s(-t) \Leftrightarrow S^{*}(f)
$$

where $*$ denotes the complex conjugate. Further, given a time delay $\tau$, Fourier transform properties dictate

$$
s(t-\tau) \Leftrightarrow S(f) e^{-j 2 \pi f \tau}
$$


Therefore, a general match-filter, given the original signal $s(t)$, with time delay $\tau$, can be written as

$$
M F(f)=S^{*}(f) e^{j 2 \pi f \tau}
$$

For each individual mode, we can assume a modal time delay $\tau_{m}(f)$ based upon the group velocity profile $v_{g m}(f)$ which can be found from

$$
\tau_{m}(f)=t_{0}(f)-\frac{R}{v_{g m}(f)},
$$

where Mode 1 serves as the reference arrival with $t_{0}(f)=R / v_{1}(f)$. Therefore, equation (C.8) can be recast into a mode-dependent matched filter,

$$
M F_{m}(f)=S^{*}(f) e^{j 2 \pi f \tau_{m}(f)}
$$

The bottom panel of Figure C.3 shows group velocity profiles for the first five modes. Figure C. 4 shows expected mode arrivals for the original chirp signal based upon this group velocity dependence. In this example, we can see the splitting at the "foot" of the chirp that was apparent in measured experimental data.

\section{C.2 Example cases}

In addition to the example figures shown in Manuscript II, Figure A.10 and Figure A.15 show non-match filtered mode arrivals stacked versus geotime. This method is particularly helpful when interpreting time-varying changes in arrival structure, such as transmissions modulated by NIW activity. Because there are breaks in these data due to SW06 acoustic source transmissions, they are broken into five groups, where arrivals are semi-consecutive. 


\section{REFERENCES AND LINKS}

1. F. B. Jensen, W. A. Kuperman, M. B. Porter and H. Schmidt, "Computational Ocean Acoustics, AIP series in modern acoustics and signal processing," American Institute of Physics, New York (1994).

2. M. B. Porter, "The KRAKEN normal mode program," Technical report, SACLANT Undersea Research Center (1992). 


\section{FIGURES}
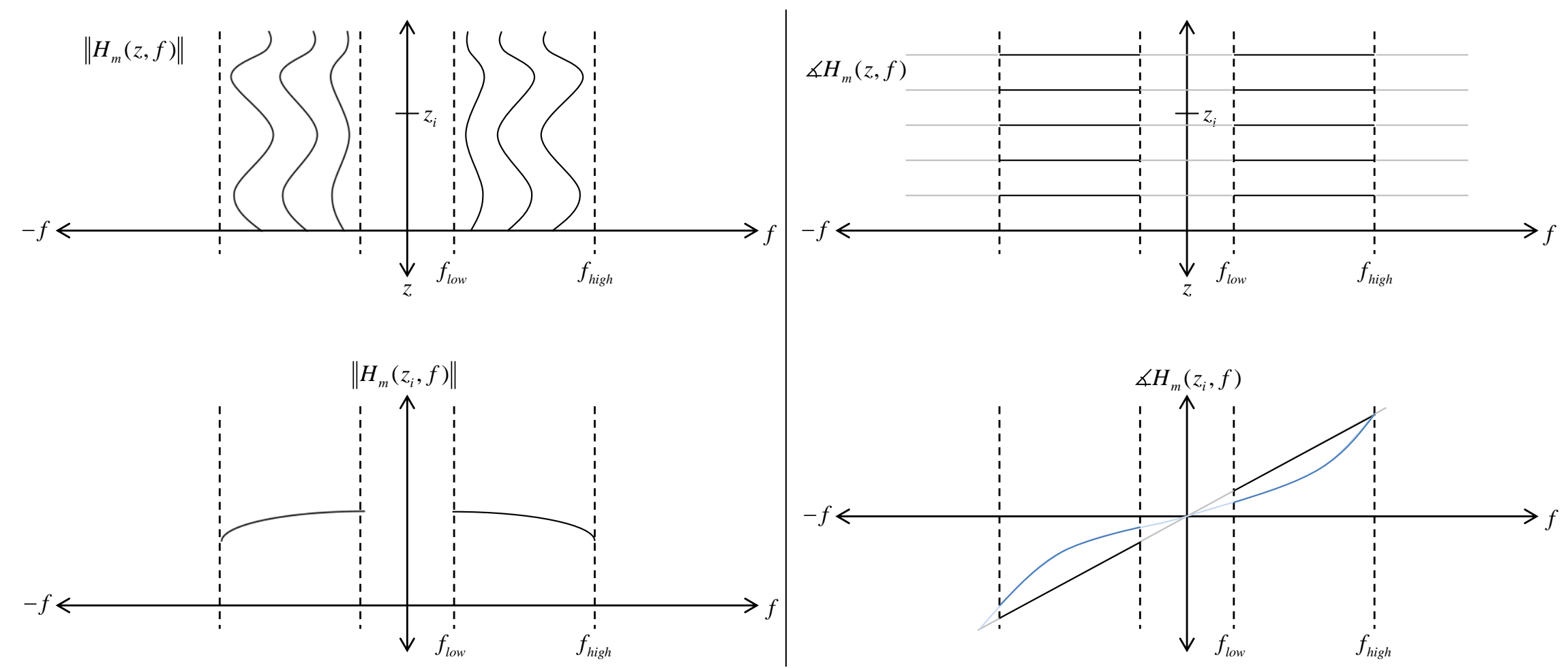

Figure C.1 Modal beamformer weighting. The magnitude of $H_{m}(z, f)$ creates a surface from the frequency dependent modeshapes (top left), corresponding to individual hydrophone weights, one example evaluated at depth $z_{i}$ (bottom left). The angle of $H_{m}(z, f)$ also creates a surface (top right) corresponding to a phase weighting at each hydrophone. A dispersive channel would show curvature, whereas a non-dispersive channel would remain linear (bottom right). 

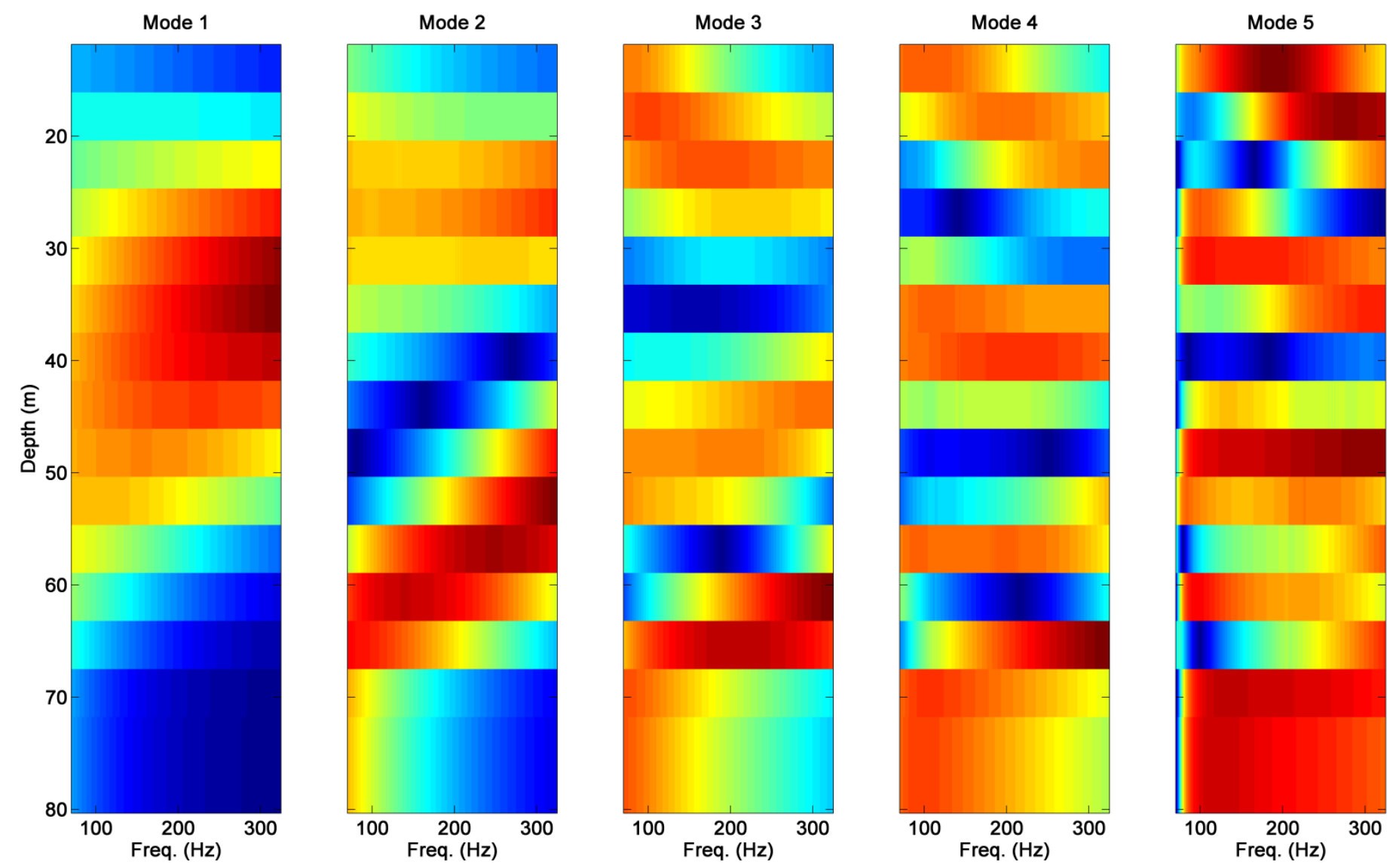

Figure C.2 One-sided amplitude weights for the first five modes, based on modeshapes calculated by the KRAKEN program 

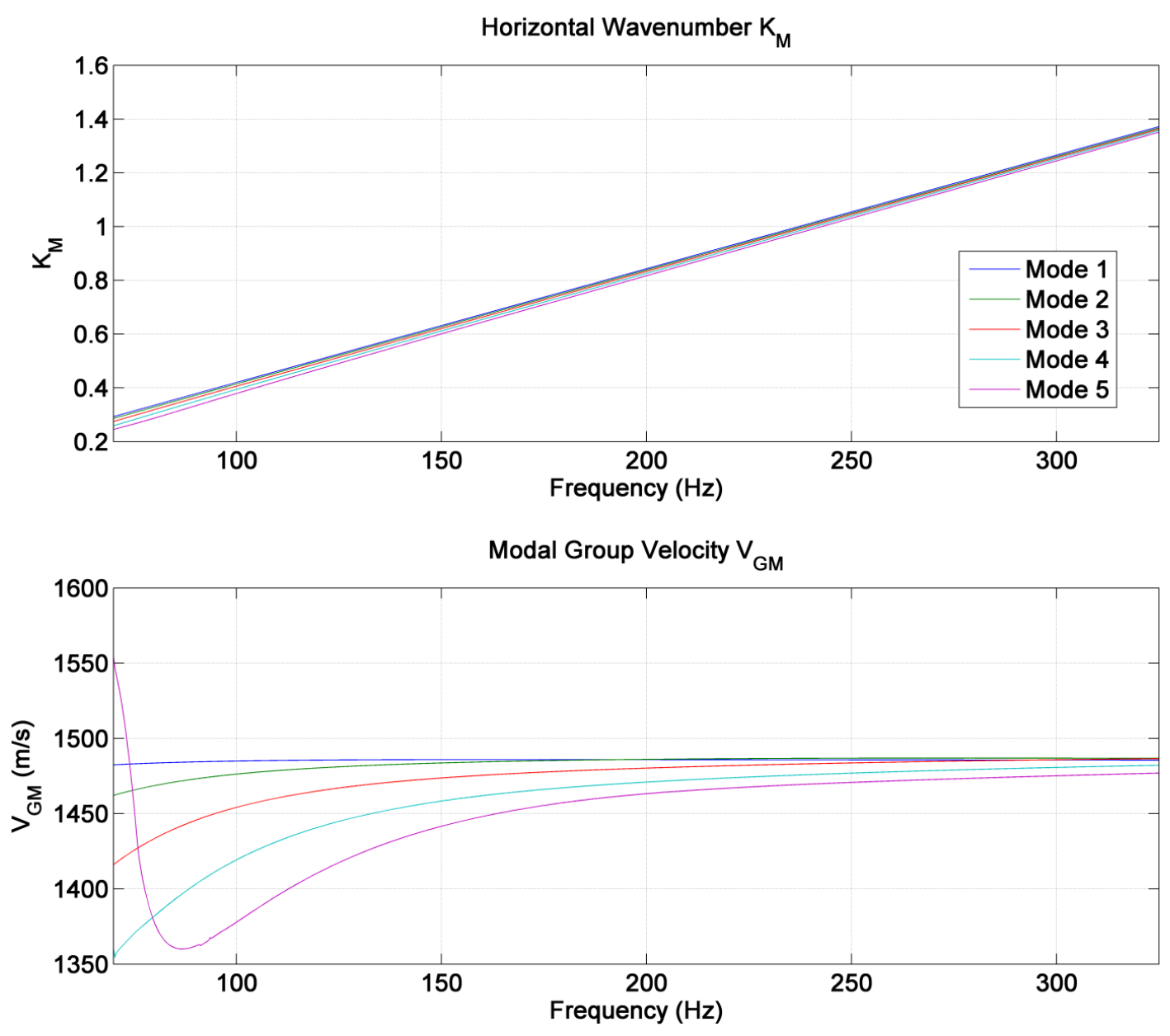

Figure C.3 Expected wavenumbers and group velocities, used to potentially calculate modal dispersion within the shallow water waveguide 

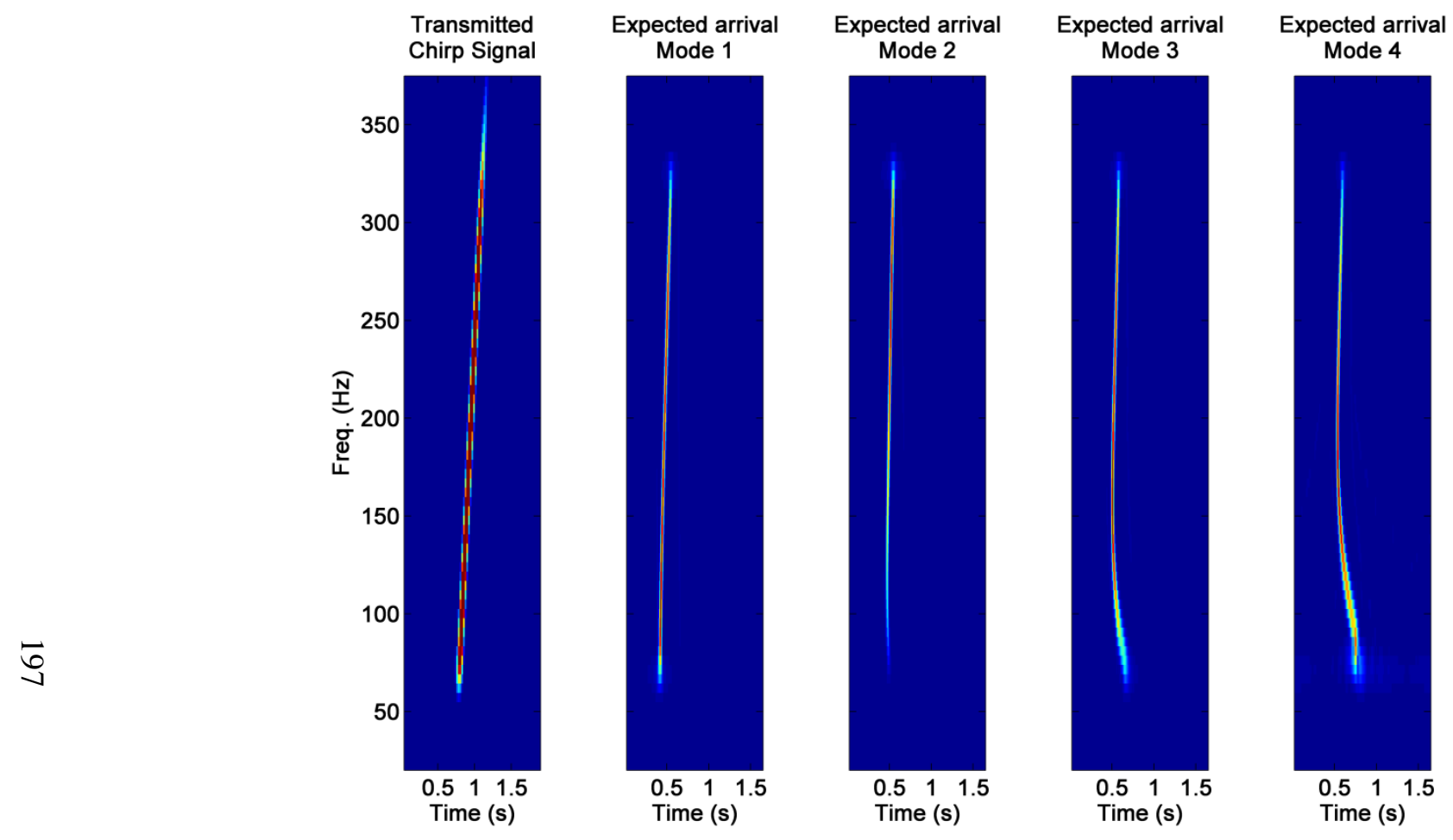

Expected arrival

$$
\text { Mode } 5
$$

Modes 1 - 5

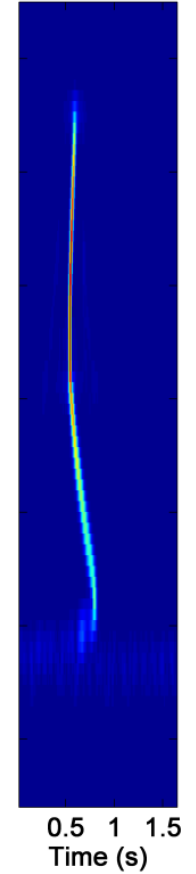

Figure C.4 Expected mode arrivals based on dispersion for the first five modes. Original transmission shown at far left, expected arrival shown at far right. 